Portland State University

PDXScholar

Fall 12-8-2017

\title{
Exploring the Determinants of Vulnerable Road Users' Crash Severity in State Roads
}

Àlvaro Alfonso Caviedes Cómbita

Portland State University

Follow this and additional works at: https://pdxscholar.library.pdx.edu/open_access_etds

Part of the Transportation Commons

Let us know how access to this document benefits you.

\section{Recommended Citation}

Caviedes Cómbita, Àlvaro Alfonso, "Exploring the Determinants of Vulnerable Road Users' Crash Severity in State Roads" (2017). Dissertations and Theses. Paper 4062.

https://doi.org/10.15760/etd.5946

This Thesis is brought to you for free and open access. It has been accepted for inclusion in Dissertations and Theses by an authorized administrator of PDXScholar. Please contact us if we can make this document more accessible: pdxscholar@pdx.edu. 
Exploring the Determinants of Vulnerable Road Users' Crash Severity in State Roads

\section{by}

Álvaro Alfonso Caviedes Cómbita

A thesis submitted in partial fulfillment of the requirements for the degree of

Master of Science

in

Civil and Environmental Engineering

Thesis Committee:

Miguel Figliozzi, Chair

Christopher Monsere

Avinash Unnikrishnan

Portland State University

2017 
(C) 2017 Álvaro Alfonso Caviedes Cómbita 


\begin{abstract}
Pedestrians and bicyclists are the most vulnerable road users and suffer the most severe consequences when crashes take place. An extensive literature is available for crash severity in terms of driver safety, but fewer studies have explored non-motorized users' crash severity. Furthermore, most research efforts have examined pedestrian and bicyclist crash severity in urban areas. This study focuses on state roads (mostly outside major urban areas) and aims to identify contributing risk factors of fatal and severe crashes involving pedestrians and bicyclists in state roads. Two ordinal regression models were developed (one for pedestrian and the other for bicyclist crashes) to examine crash severity risk factors. Additional models were developed to investigate road and traffic characteristics that could increase the likelihood of fatal crashes. In the model for pedestrian crash severity risk factors such as age, vehicle type and movement, light conditions, road classification, traffic control device, posted speed limit, location of the pedestrian and wet road surface during clear weather conditions are statistically significant. The bicyclist crash severity model indicates that age, crash location, vehicle movement and alcohol intoxication during dark conditions are statistically significant. In terms of road characteristics and traffic conditions, the models suggested risk factors such as arterials, light conditions, posted speed limit, roadways, and high heavy vehicle volume, increased the odds of a crash being fatal.

The results seem to suggest that besides improvements in roadway characteristics, additional countermeasures to reduce crash severity for vulnerable users should include
\end{abstract}


separation of vulnerable users from traffic, educational campaigns, more strict control of alcohol intoxicated drivers, and protection strategies of senior pedestrians. 


\section{ACKNOWLEDGMENTS}

I would like to express my sincerest appreciation to Dr. Figliozzi for all his support and academic guidance in this research and my time in Portland State University. I would also like to thank Dr. Monsere and Dr. Unnikirshnan for their assistance, collaborators and members on the supervisory committee for this thesis. The author also recognize the support of members of the TTP laboratory at PSU College of Engineering who contributed with their feedback. Any errors or omissions are the sole responsibility of the authors. I would like to thank Alexa Johnson for her continued support and assistance as a reviewer of the document. Finally, I would like to thank my family who unconditionally supported me during this long process back in Colombia. 


\section{TABLE OF CONTENTS}

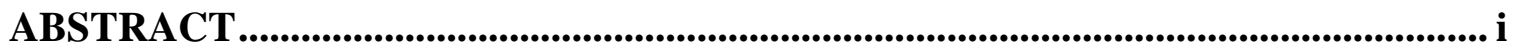

ACKNOWLEDGMENTS …..............................................................................

LIST OF TABLES …...........................................................................................

LIST OF FIGURES …....................................................................................... vii

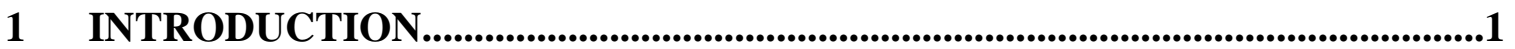

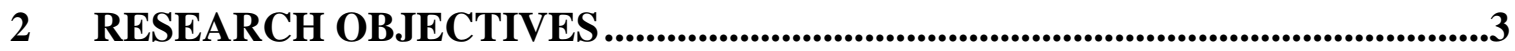

3 LITERATURE REVIEW ......................................................................................4

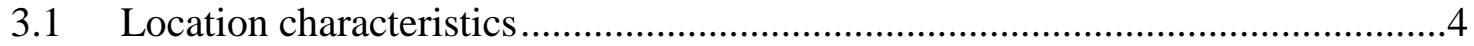

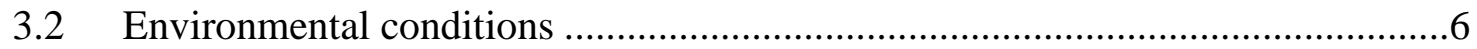

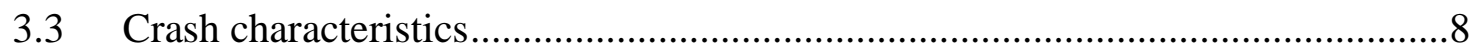

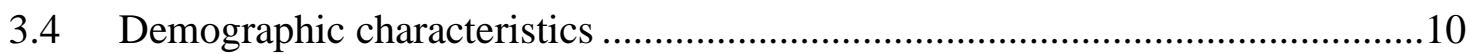

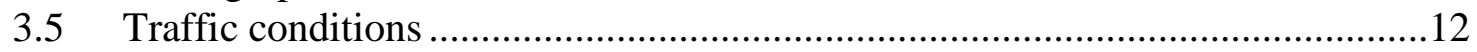

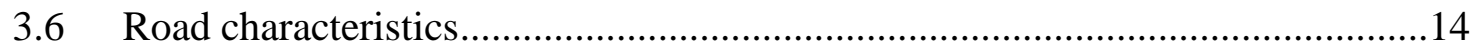

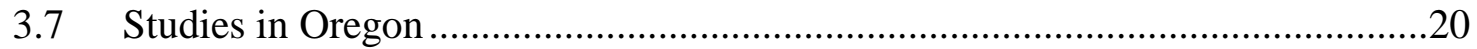

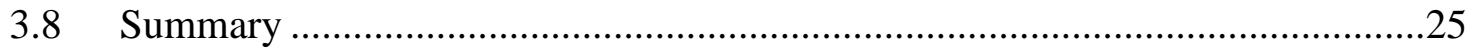

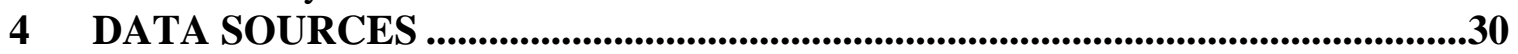

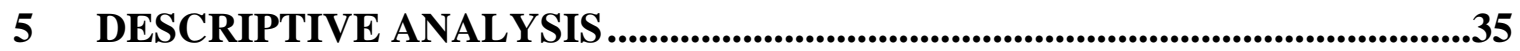

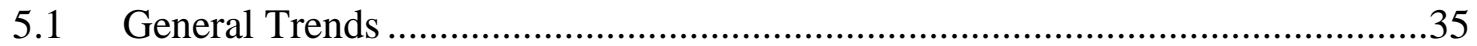

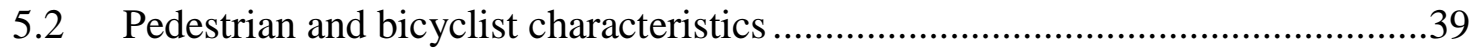

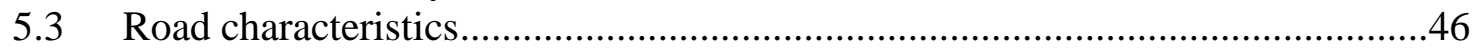

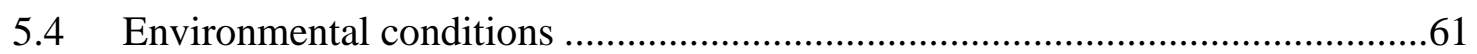

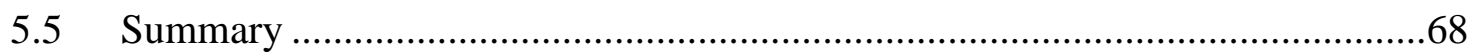

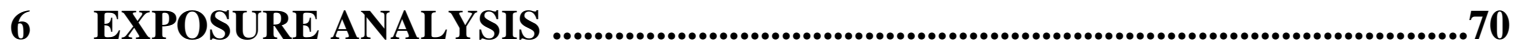

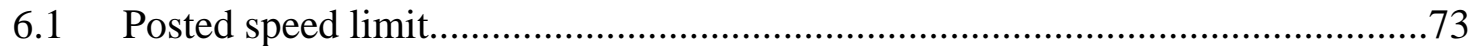

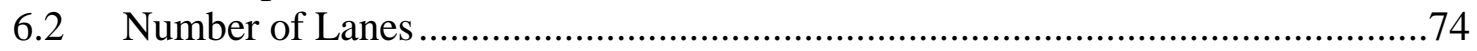

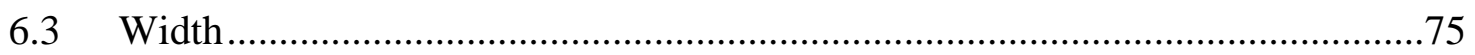

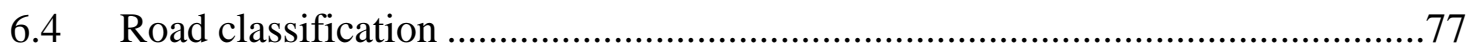

6.5 Neighborhood Concepts .........................................................................

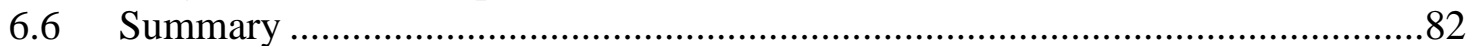

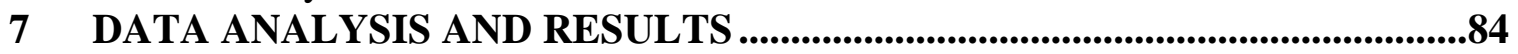

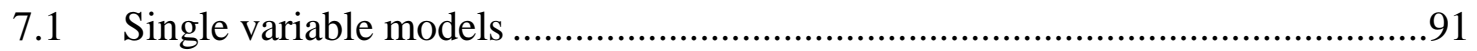

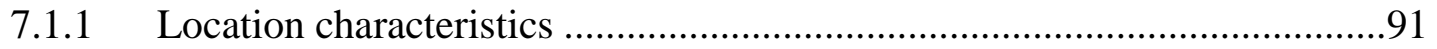

7.1.2 Environmental conditions …………………........................................91

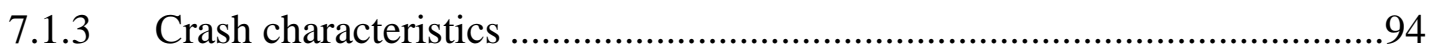

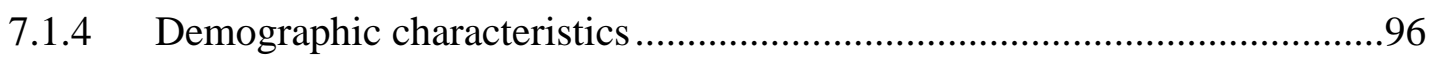

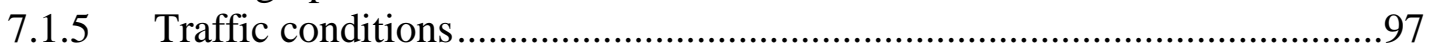

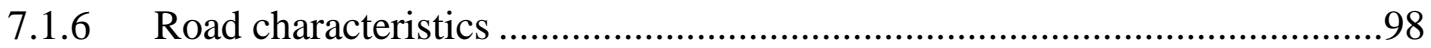

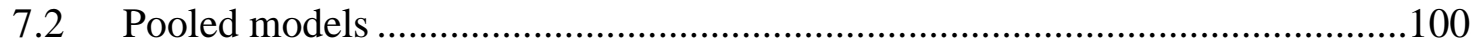

7.3 Road characteristics and traffic conditions models ...........................................109

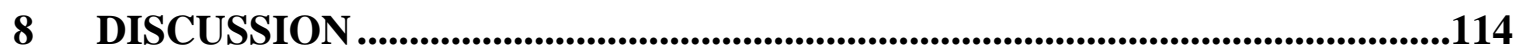

9 POLICY IMPLICATIONS....................................................................................120

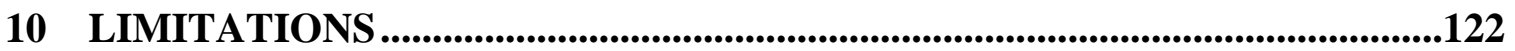




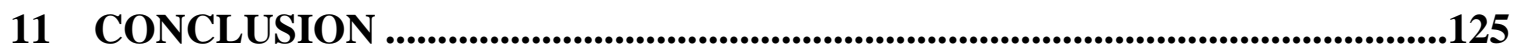

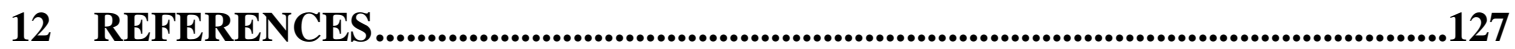

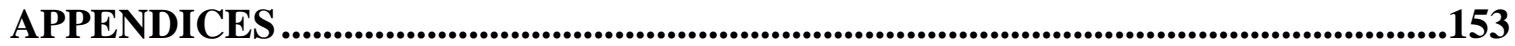

Appendix A: risk ratio by neighborhood concept ..................................................153

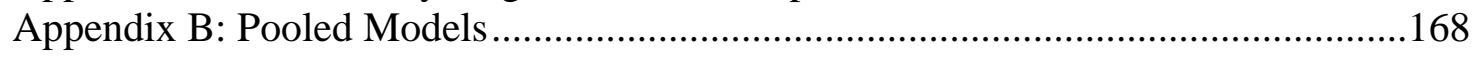

Appendix C: Road characteristics and traffic conditions models ............................171 


\section{LIST OF TABLES}

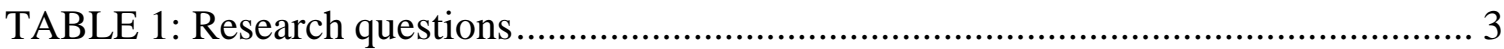

TABLE 2: Summary of location characteristics review .................................................. 6

TABLE 3: Summary of environmental conditions review ............................................. 7

TABLE 4: Summary of crash characteristics review ………………………………...... 9

TABLE 5: Summary of demographic characteristics review ………………………...... 12

TABLE 6: Summary of traffic conditions review ....................................................... 13

TABLE 7: Summary of road characteristics review ................................................... 18

TABLE 8: ODOT Pedestrian and Bicycle Safety Implementation Plan findings ............. 21

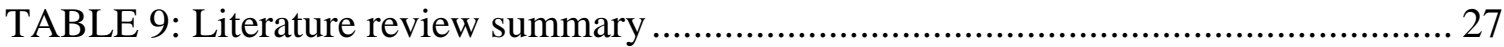

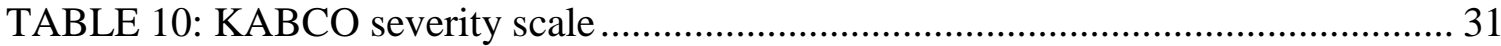

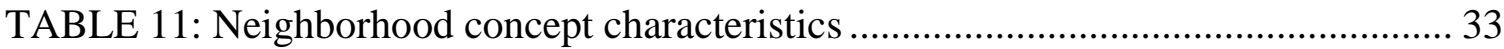

TABLE 12: Statewide pedestrian crashes by neighborhood concepts and year............... 36

TABLE 13: Statewide bicyclist crashes by neighborhood concepts and year.................. 38

TABLE 14: Statewide pedestrian crashes (2007-2014) by severity level, neighborhood concept and vulnerable user age. ............................................................................... 40

TABLE 15: Statewide bicyclist crashes (2007-2014) by severity level, neighborhood

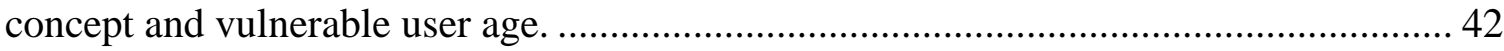

TABLE 16: Statewide pedestrian crashes (2007-2014) by severity level, neighborhood concept and vulnerable user gender.............................................................................. 44

TABLE 17: Statewide bicyclist crashes (2007-2014) by severity level, neighborhood

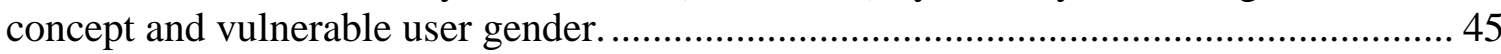

TABLE 18: Statewide pedestrian crashes (2007-2014) by severity level, neighborhood

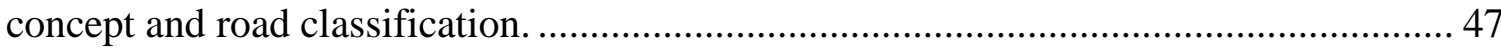

TABLE 19: Statewide bicyclist crashes (2007-2014) by severity level, neighborhood concept and road classification.

TABLE 20: Statewide pedestrian crashes (2007-2014) by severity level, neighborhood concept and posted speed limit.

TABLE 21: Statewide bicyclist crashes (2007-2014) by severity level, neighborhood concept and posted speed limit.

TABLE 22: Statewide pedestrian crashes (2007-2014) by severity level, neighborhood concept and number of lanes.

TABLE 23: Statewide bicyclist crashes (2007-2014) by severity level, neighborhood concept and number of lanes

TABLE 24: Statewide pedestrian crashes (2007-2014) by severity level, neighborhood concept and road surface.

TABLE 25: Statewide bicyclist crashes (2007-2014) by severity level, neighborhood concept and road surface.

TABLE 26: Statewide pedestrian crashes (2007-2014) by severity level, neighborhood concept and weather conditions

TABLE 27: Statewide bicyclist crashes (2007-2014) by severity level, neighborhood concept and weather conditions

TABLE 28: Statewide pedestrian crashes (2007-2014) by severity level, neighborhood concept and light conditions 
TABLE 29: Statewide bicyclist crashes (2007-2014) by severity level, neighborhood

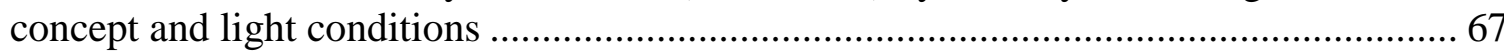

TABLE 30: Pedestrian and bicyclist crashes in Oregon................................................ 70

TABLE 31: Pedestrian crash frequency and posted speed limit exposure ratio ................ 73

TABLE 32: Bicyclist crash frequency and posted speed limit exposure ratio .................. 73

TABLE 33: Proportion of road segment by posted speed limit....................................... 74

TABLE 34: Pedestrian crash frequency and number of lanes exposure ratio ................... 74

TABLE 35: Bicyclist crash frequency and number of lanes exposure ratio....................... 75

TABLE 36: Proportion of road segment by number of lanes ....................................... 75

TABLE 37: Pedestrian crash frequency and width exposure ratio..................................... 76

TABLE 38: Bicyclist crash frequency and width exposure ratio ..................................... 76

TABLE 39: Proportion of road segment by road width ................................................ 77

TABLE 40: Pedestrian crash frequency and road classification exposure ratio................ 77

TABLE 41: Bicyclist crash frequency and road classification exposure ratio .................. 78

TABLE 42: Proportion of road segment by road classification ...................................... 78

TABLE 43: Crash frequency and neighborhood concept exposure ratio summary ......... 79

TABLE 44: Proportion of road segment by neighborhood concepts .............................. 82

TABLE 45: Exposure analysis summary ................................................................... 83

TABLE 46: Distribution (\%) of risk factors for different severity levels.......................... 86

TABLE 47: Single variable model - location characteristics (odd ratios) ....................... 91

TABLE 48: Single variable model - environmental conditions (odd ratios) .................... 92

TABLE 49: Single variable model - crash characteristics (odd ratios)............................ 95

TABLE 50: Single variable model - demographic characteristics (odd ratios) …............ 97

TABLE 51: Single variable model - traffic conditions (odd ratios) ………………......... 98

TABLE 52: Single variable model - road characteristics (odd ratios) ............................. 99

TABLE 53: AADT only trucks cut-points.................................................................... 101

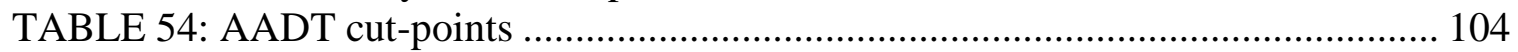

TABLE 55: Ordinal regression model. Crash severity for pedestrians and bicyclists ... 105

TABLE 56: Predictive power rank ......................................................................... 108

TABLE 57: Ordinal regression model. Road and traffic characteristics .......................... 111

TABLE 58: Predictive power rank (road and traffic characteristics) ............................. 113

TABLE 59: Impact of risk factors on severity level................................................... 117

TABLE 60: Impact of road and traffic risk factors on severity level ............................. 119 


\section{LIST OF FIGURES}

FIGURE 1: (a) Pedestrian and (b) Bicyclist crashes by location. This figure is adapted from Kittelson \& Associates, Inc. (2014)

FIGURE 2: (a)Pedestrian and (b)Bicyclist crashes by road characteristics and intersection type. This figure is adapted from Kittelson \& Associates, Inc. (2014) ......................... 24

FIGURE 3: Crash event processing ..................................................................... 31

FIGURE 4: Neighborhood Concept Types, adapted from Currans et al. (2015)............ 32 FIGURE 5: Oregon Neighborhood Concept, (a) State of Oregon, (b) Portland Metro Area

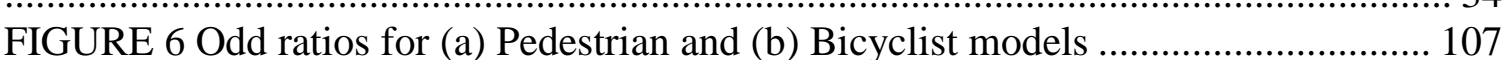

FIGURE 7 Odd ratios for (a) Pedestrian and (b) Bicyclist models (only road and traffic

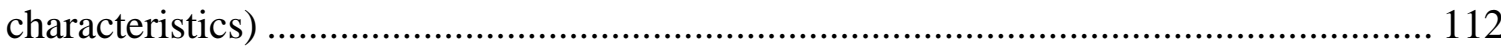




\section{INTRODUCTION}

There is a growing interest in increasing non-motorized transportation in the state of

Oregon as a strategy to improve public health, air quality, and to reduce traffic

congestion. With an expected increase of $30 \%$ in the total population by 2040 , the state of Oregon expects to continue its growth in walking and biking (Oregon Department of Transportation, 2016). As residents in Oregon choose non-motorized modes, safe walking and biking facilities become a priority.

At the national level, $41 \%$ of trips under three miles are made by walking or bicycle (U.S. Department of Transportation, 2015), unfortunately there have been an increase in fatalities and serious injuries for bicyclists and pedestrians (U.S. Department of Transportation, 2015).

Pedestrians and bicyclists are among the most vulnerable users on the road and in crashes with vehicles they tend to suffer the most severe consequences due to their lack of protection. Risk factors associated to crash severity for pedestrians and bicyclists have been understudied in suburban and rural roads, making it difficult for Department of Transportation agencies (DOT) to implement countermeasures and policies to improve safety for vulnerable road users.

This study aims to identify contributing risk factors of fatal and severe crashes involving pedestrians and bicyclists in Oregon DOT (ODOT) roads. Identification of these factors will provide ODOT with the ability to implement appropriate countermeasures to reduce crash severity for pedestrians and bicyclists.

This research used pedestrian and bicyclist crash data for the years 2007 to 2014 collected from the Oregon Statewide Crash Data System. Additional information on the 
ODOT's highway network, traffic conditions and road characteristics was gathered from the Oregon Department of Transportation's TransGIS database.

The remainder of the paper is structured as follows. First, we discuss the main objectives of this study and the extent of the research. Second, we show a literature review of risk factors and crash severity for pedestrian and bicyclists. Then, a description of the data sources used on this study is presented. In section 5 we present a descriptive analysis of risk factors for severity, and its relationship with different land uses. This section is followed by an exposure analysis, intended to uncover crash severity patterns by controlling by VMT and land use. Section 7 presents a brief overview of the statistical model used in this research. Then, in section 8 we present the data analysis performed, split by single variable models and pooled modes (using all the variables, and then only road and traffic risk factors). Finally, we discuss the findings and then we close with limitations and conclusions. 


\section{RESEARCH OBJECTIVES}

TABLE 1 outlines the research questions this thesis addresses and the different methodologies used to explore them.

\section{TABLE 1: Research questions}

\begin{tabular}{c|c}
\hline \hline RESEARCH QUESTION & METHODOLOGIES \\
\hline $\begin{array}{c}\text { What are the main risk factors associated to } \\
\text { pedestrian and bicyclist crash severity? }\end{array}$ & $\begin{array}{c}\text { Literature review } \\
\text { Descriptive analysis }\end{array}$ \\
\hline $\begin{array}{c}\text { How crash severity levels are affected by } \\
\text { exposure of pedestrians and bicyclists? }\end{array}$ & $\begin{array}{c}\text { Descriptive analysis } \\
\text { Exposure analysis }\end{array}$ \\
\hline $\begin{array}{c}\text { How location, environmental, crash, } \\
\text { demographic traffic, and road characteristics } \\
\text { increase or decrease severity levels for } \\
\text { vulnerable users in state roads? }\end{array}$ & $\begin{array}{c}\text { Exposure analysis } \\
\text { Descriptive analysis }\end{array}$ \\
$\begin{array}{c}\text { What are the main risk factors associated with } \\
\text { severity levels for vulnerable users in state } \\
\text { roads? }\end{array}$ & $\begin{array}{c}\text { Ordinal logistic regression (single variable } \\
\text { models) }\end{array}$ \\
Ordinal logistic regression (pooled models) \\
\hline $\begin{array}{c}\text { What are the main risk factors associated to } \\
\text { road and traffic characteristics? }\end{array}$ & $\begin{array}{c}\text { Sensitivity analysis (pooled models) } \\
\text { Ordinal logistic regression (road and traffic char. } \\
\text { models) }\end{array}$ \\
& $\begin{array}{c}\text { Sensitivity analysis (road and traffic char. } \\
\text { models) }\end{array}$ \\
\hline
\end{tabular}




\section{LITERATURE REVIEW}

Although there is extensive research in crash severity in terms of driver safety, studies on non-motorized users is more limited. Researchers who have studied vulnerable users identified several main risk factors that contribute to severity. These factors were classified into six major categories: location characteristics, environmental conditions, crash, demographic characteristics, road, and traffic conditions. This section reviews different risk factors that several researchers have identified. Furthermore, we mentioned some of the methodologies they have used as a reference for the present study.

\subsection{Location characteristics}

Zahabi et al. (2011) developed several models to determine the effects of road design, build environment, speed limits, and other factors on crash severity with pedestrians and cyclists involved. Data on crashes, land use (50 to 400 meters from the crash location), and road characteristics were collected for the city of Montreal. The authors found that after including land use variables in the model, the overall power of the explanatory variables increased. Pedestrian crashes that occurred near a park tended to be more severe than crashes at other locations, while pedestrian crashes that occurred near a school tended to be less severe. This finding can be attributed to the safety measures that were taken near schools.

Opposite results were found by Kim et al (2007). For bicyclist crashes, the findings suggested that areas with a school increased the probability of incapacitating injury; however, it decreases the likelihood for other severity levels (Kim, Kim, G., \& Porello, 2007). 
Abdul-Aziz et al. (2013) found that land use designated as industrial is associated with less severe and fatal pedestrian crashes. This finding is not surprising if we consider the low levels of pedestrian activity in these zones. On the other hand, the authors found a significant effect of commercial neighborhoods in crash severity.

Islam and Hossain (2015) explored the influence of different risk factors in severity of crashes involving non-motorized users. The authors employed a mixed-logit model approach. Severity was defined used the KABCO scale, but adding $\mathrm{C}$ and $\mathrm{O}$ in one category itself. A total of 2,442 and 789 observations for pedestrian and bicycle crashes, respectively, were analyzed. The findings suggested that occurrence of crashes in residential areas tended to increase the probability of a severe injury for both pedestrians and bicyclists. Commercial land use was also statistically significant; however, for bicyclist crashes, it had an opposite direction, crashes at commercial areas tended to be less severe and fatal than in other areas.

TABLE 2 summarizes the studies that found relationships between pedestrian and bicyclist severity levels and vulnerable user's characteristics. The arrows show the direction of the relationship between the independent variables and pedestrian severity. When an upward pointing arrow symbol $(\uparrow)$ is used, the probability for the crash to be fatal or severe increases due to the variable studied. When a downward pointing arrow symbol $(\downarrow)$ is used, the probability for the crash to be fatal or severe decreases. When the symbol is a dash (-), the study did not find a statistical difference. 
TABLE 2: Summary of location characteristics review

\begin{tabular}{|c|c|c|c|c|}
\hline \multicolumn{5}{|c|}{ PEDESTRIAN STUDIES } \\
\hline Author (year) & $\begin{array}{l}\text { Transit } \\
\text { access }\end{array}$ & $\begin{array}{c}\text { Commercial land } \\
\text { use }\end{array}$ & $\begin{array}{c}\text { Park } \\
\text { presence }\end{array}$ & $\begin{array}{c}\text { School } \\
\text { presence }\end{array}$ \\
\hline Clifton et al. (2009) & $\downarrow$ & & & \\
\hline Zahabi et al. (2011) & $\uparrow$ & $\uparrow$ & $\uparrow$ & $\downarrow$ \\
\hline Seyed et al. (2011) & & & & $\downarrow$ \\
\hline $\begin{array}{c}\text { Abdul-Aziz et al. } \\
\text { (2013) }\end{array}$ & & $\bar{\uparrow}$ & & \\
\hline $\begin{array}{c}\text { Islam and Hossain } \\
(2015)\end{array}$ & & $\uparrow$ & & \\
\hline \multicolumn{5}{|c|}{ BICYCLIST STUDIES } \\
\hline Zahabi et al. (2011) & - & - & - & - \\
\hline $\begin{array}{c}\text { Islam and Hossain } \\
(2015)\end{array}$ & & $\downarrow$ & & \\
\hline Kim et al. (2007) & & & & $\uparrow$ \\
\hline
\end{tabular}

\subsection{Environmental conditions}

As expected, lighting conditions were associated with crash severity. Good lighting conditions resulted in less severe pedestrian injuries (Zheng, 2014 ). Similar findings were found in New York (Abdul-Aziz, Ukkusuri, \& Hasan, 2013). Chen et al. (2016) compared severity risk factors for crashes sustained by pedestrians in facing-traffic and back-to-traffic. A binary logit mode was used to evaluate the outcome of the injury, where the injury was located (e.g. head), and if the road was built or not built. The findings of this study suggested that pedestrian crashes during dark conditions and during morning peak hours were more likely to result in an injury. For bicyclist crashes the trend was similar, better lighting conditions improved visibility, which reduced the likelihood of a severe and fatal crash (Allen-Munley, Daniel, \& Dhar, 2004).

Several studies have demonstrated that inclement weather conditions, such as rain, snow and fog, were strongly associated with crash severity levels. Li et al. (2016) explored the impact of pedestrian and driver characteristics on crash severity under 
different weather conditions. Crash data from Great Britain was used for this analysis. A total of 14,174 crashes were assessed. The authors used nonparametric methods (classification and regression tree) to develop a model of crash severity. Under fine weather conditions, high-posted speed limits, older pedestrians, and poor light conditions, the likelihood of a severe crash increased. Under inclement weather conditions, only posted speed limit and older pedestrians were found to be predictors of severe crashes. Inclement weather conditions were significantly associated with bicyclist injury severity as well (Kim, Kim, G., \& Porello, 2007). Similar to lighting conditions variables, the authors suggested that weather was a confounding effect for other unobservable variables, such as visibility and bicycle brake failures.

In terms of the day of the week, the study conducted by Kim et al. (2007) revealed that bicyclists were more likely to be injured during the weekend than on weekdays. Additionally, for crashes occurring on weekday peak hours, the severity levels tended to be higher as well.

TABLE 3: Summary of environmental conditions review

\begin{tabular}{|c|c|c|c|c|}
\hline \multicolumn{5}{|c|}{ PEDESTRIAN STUDIES } \\
\hline Author (year) & $\begin{array}{l}\text { Inclement } \\
\text { weather } \\
\text { conditions }\end{array}$ & $\begin{array}{l}\text { Night time/ } \\
\text { poor lighting } \\
\text { conditions }\end{array}$ & $\begin{array}{c}\text { Peak } \\
\text { hours }\end{array}$ & $\begin{array}{c}\text { Weekend } \\
\text { days }\end{array}$ \\
\hline Tay et al. (2011) & $\uparrow$ & $\uparrow$ & $\uparrow$ & \\
\hline Zheng (2014) & & $\uparrow$ & & \\
\hline Zhang et al. (2014) & & $\uparrow$ & & $\uparrow$ \\
\hline Jang et al. (2013) & $\uparrow$ & & & $\uparrow$ \\
\hline Haleem et al. (2015) & $\uparrow$ & $\uparrow$ & & \\
\hline Chen et al. (2016) & & $\uparrow$ & $\uparrow$ & \\
\hline Eluru et al. (2008) & & $\uparrow$ & & \\
\hline Li et al. (2016) & & $\uparrow$ & & \\
\hline Islam and Hossain (2015) & $\uparrow$ & $\uparrow$ & & \\
\hline Abdul-Aziz et al. (2013) & & $\uparrow$ & & \\
\hline McIntyre (2016) & & $\uparrow$ & & \\
\hline Zahabi et al. (2001) & & $\uparrow$ & & \\
\hline
\end{tabular}


TABLE 3: Summary of environmental conditions review, continued

\begin{tabular}{c|c|c|c|c}
\hline \hline \multicolumn{5}{c}{ BICYCLIST STUDIES } \\
\hline Author (year) & $\begin{array}{c}\text { Inclement } \\
\text { weather } \\
\text { conditions }\end{array}$ & $\begin{array}{c}\text { Night time/ } \\
\text { poor lighting } \\
\text { conditions }\end{array}$ & $\begin{array}{c}\text { Peak } \\
\text { hours }\end{array}$ & $\begin{array}{c}\text { Weekend } \\
\text { days }\end{array}$ \\
\hline Kim et al. (2007) & $\uparrow$ & $\uparrow$ & $\uparrow$ & $\uparrow$ \\
\hline Islam and Hossain (2015) & $\uparrow$ & $\uparrow$ & & \\
\hline Allen-Munley et al.(2004) & & $\uparrow$ & & \\
\hline McIntyre (2016) & $\downarrow$ & & & \\
\hline Eluru et al. (2008) & & $\uparrow$ & & \\
\hline \hline
\end{tabular}

\subsection{Crash characteristics}

There was a consensus between the different studies in terms of crash severity and type of vehicles. A crash that involved a pedestrian (or a bicyclist) and a heavy vehicle was more likely to be fatal or severe than a crash with a lighter vehicle. Zheng (2014) also revealed that heavy vehicles had a higher crash rate than light vehicles, which increased the overall risk for pedestrians. Zahabi (2011) found that vehicle size was positively associated with pedestrian and bicyclist severity; however, the coefficient was only statistically significant for pedestrian crashes. On the other hand, Kim et al. (2007), Eluru et al. (2008), and Allen-Munley et al. (2004) found that heavy trucks increased the probability of severe and fatal crashes compared to other types of vehicles. Eluru et al. (2008) explained that non-motorist crashes with heavy vehicles tended to be fatal or severe due to higher bumper heights and impact areas.

Multiple studies have also explored the impact of alcohol on crash severity; however, most of these studies did not consider pedestrians and bicyclists exclusively. Kim et al. (1995) found that the odds of a head-on or rollover collision were much greater 
in the presence of alcohol or drugs. Additionally, young people were less likely to crash under alcohol effects than old people were.

Eluru et al. (2008) developed a mixed generalized ordered response logit to explore severity in crashes involving pedestrians and bicyclists. The authors found that pedestrians and bicyclists who were hit by an alcohol-intoxicated driver were more prone to be severely injured. Similarly, Kim et al. (2008) found that intoxicated drivers increased the probability of fatal injury by $17 \%$ and incapacitating injury by $24 \%$. Lee and Abdel-Aty (2003) explored the interaction between lighting conditions and impaired drivers and found that people who were driving under alcohol effects in dark conditions tended to be involved in more severe crashes than people driving during dark conditions with street lighting are.

TABLE 4: Summary of crash characteristics review

\begin{tabular}{c|c|c}
\hline \hline & \multicolumn{2}{c}{ PEDESTRIAN STUDIES } \\
\hline $\begin{array}{c}\text { Lee and Abdel-Aty } \\
\text { (2003) }\end{array}$ & Size of the vehicle & Alcohol intoxication \\
\hline Tay et al. (2011) & $\uparrow$ & $\uparrow$ \\
\hline Zheng (2014) & $\uparrow$ & \\
\hline Seyed et al. (2011) & $\uparrow$ & \\
\hline $\begin{array}{c}\text { Tarko and Azam } \\
\text { (2011) }\end{array}$ & $\uparrow$ & \\
\hline $\begin{array}{c}\text { Haleem et al. } \\
\text { (2015) }\end{array}$ & $\uparrow$ & \\
\hline Eluru et al. (2008) & $\uparrow$ & \\
\hline $\begin{array}{c}\text { Allen-Munley et } \\
\text { al. (2004) }\end{array}$ & $\uparrow$ & \\
\hline Eluru et al. (2008) & $\uparrow$ & \\
\hline Matsui and & $\uparrow$ & \\
Oikawa (2016) & $\uparrow$ & \\
\hline $\begin{array}{c}\text { Zahabi et al. } \\
(2011)\end{array}$ & $\uparrow$ & \\
\hline $\begin{array}{c}\text { Sasidharan et al. } \\
\text { (2015) }\end{array}$ & $\uparrow$ & \\
\hline Kim et al. (1995) & & \\
\hline \hline
\end{tabular}


TABLE 4: Summary of crash characteristics review, continued

\begin{tabular}{c|c|c}
\hline \hline \multicolumn{2}{c|}{ BICYCLIST STUDIES } & \\
\hline Author (year) & Size of the vehicle & Alcohol intoxication \\
\hline $\begin{array}{c}\text { Zahabi et al. } \\
(2011)\end{array}$ & - & \\
\hline Kim et al. (2007) & $\uparrow$ & \\
\hline $\begin{array}{c}\text { Allen-Munley et } \\
\text { al.(2004) }\end{array}$ & $\uparrow$ & \\
\hline Eluru et al. (2008) & $\uparrow$ & $\uparrow$ \\
\hline \hline
\end{tabular}

\subsection{Demographic characteristics}

Two variables were used for pedestrian characteristics, age and gender. Most of the studies classified age into different categories, defining elder pedestrian as people over the age of 65 .

Tay et al. (2011) used a multinomial logit model to estimate the likelihood of a pedestrian being involved in a fatal, severe and non-severe crash. The authors chose this approach because it allowed them to model an unordered response and a non-monotonic effect of the independent variables on the dependent variable. A 2006 database from the South Korean Police agency was used for this purpose. The findings suggested that elder pedestrians had a greater likelihood of being involved in a fatal and severe crash than younger pedestrians. Kim et al. (2008) studied police reported crash data between 1997 and 2000 to investigate the relationship of age in the severity of the crash. The authors found that as the people get older, the probability of fatal or severe injuries after a crash increased. Similarly, Zheng (2014 ) showed that elder pedestrians are at a higher risk of a severe crash than younger pedestrians. These findings were attributed to the fact that walking speed, visibility reaction time, and body resistance tend to be lower for this demographic group. Kim et al. (2007) explored the factors that contributed to bicyclist 
severity levels due to a crash with a motorized vehicle. A total of 2.937 bicyclist crashes from the North Carolina police report database (1997 to 2002) were used. Bicyclists with an age of 55 and over had a greater likelihood of being involved in a fatal or injury crash than younger bicyclists.

Zhao et al. (2014), a team of engineers and medical experts, collected crash data (2006) in the city of Beijing and Northern China. Only 121 crashes were studied. The study controlled for impact speed, which was estimated from the vehicle's breaking distance before the collision. Additional to the data regarding the crash, the researchers included injured body region, treatment type after the crash and surviving time. Severity was measured based on the Abbreviated Injury Scale 2005, which goes from AIS 1 (minor injury) to AIS 6 (currently untreatable). After controlling severity for the pedestrian's body impact location, the authors did not find a difference in severity levels between male and female pedestrians. Sze and Wong (2007) evaluated injury risk factors of pedestrian fatalities in Hong Kong. This study measured the associations between the injury level of pedestrian causalities and all possible contributory factors such as gender, crash characteristics, location, crash time, traffic characteristics, road environment, speed limit, road type, traffic conditions and junction controls. Furthermore, the authors included a variable to control for the temporal variation of road infrastructure and vehicle technology improvements. The findings suggested that being a male increased the chances of a fatal or severe crash. Islam and Hossain (2015) found similar results, bicyclist and pedestrian males tended to be involved in more severe and fatal crashes as well. On the other hand, it is interesting to notice that driver age had an opposite effect on 
the severity of the crash (not shown in (TABLE 5). Senior drivers tended to be more cautious than young drivers, which resulted in less fatal crashes (Zheng, 2014 ).

TABLE 5: Summary of demographic characteristics review

\begin{tabular}{|c|c|c|c|c|}
\hline \multicolumn{4}{|c|}{ PEDESTRIAN STUDIES } & \multirow[b]{2}{*}{$\begin{array}{l}\text { Alcohol } \\
\text { effects }\end{array}$} \\
\hline Author (year) & $\begin{array}{c}\text { Young } \\
\text { pedestrians } \\
(<15)\end{array}$ & $\begin{array}{c}\text { Older pedestrians } \\
(>65)\end{array}$ & $\begin{array}{c}\text { Male } \\
\text { pedestrian }\end{array}$ & \\
\hline $\begin{array}{l}\text { Wazana et al. } \\
(2000)\end{array}$ & $\uparrow$ & & & \\
\hline Tay et al. (2011) & & $\uparrow$ & $\downarrow$ & \\
\hline Zhao et al. (2014) & & & - & \\
\hline Kim et al. (2008) & & $\uparrow$ & & $\uparrow$ \\
\hline Zheng (2014) & & $\uparrow$ & & \\
\hline $\begin{array}{c}\text { Zhang et al. } \\
\text { (2014) }\end{array}$ & & & - & \\
\hline $\begin{array}{c}\text { Haleem et al. } \\
(2015)\end{array}$ & & $\uparrow$ & & \\
\hline $\begin{array}{c}\text { Islam and } \\
\text { Hossain (2015) }\end{array}$ & & $\uparrow$ & & \\
\hline Eluru et al. (2008) & & $\uparrow$ & $\uparrow$ & $\uparrow$ \\
\hline McIntyre (2016) & $\uparrow$ & $\uparrow$ & & \\
\hline Li et al. (2016) & & $\uparrow$ & & \\
\hline $\begin{array}{c}\text { Sze and Wong } \\
(2007)\end{array}$ & $\downarrow$ & $\uparrow$ & $\downarrow$ & \\
\hline $\begin{array}{c}\text { Abdul-Aziz et al. } \\
(2013)\end{array}$ & & $\uparrow$ & - & \\
\hline $\begin{array}{c}\text { Islam and } \\
\text { Hossain (2015) }\end{array}$ & & $\uparrow$ & $\uparrow$ & \\
\hline McIntyre (2016) & & $\uparrow$ & & \\
\hline $\begin{array}{c}\text { Lee and Abdel- } \\
\text { Aty (2003) }\end{array}$ & & & & $\uparrow$ \\
\hline Kim et al. (1995) & & & & $\uparrow$ \\
\hline \multicolumn{5}{|c|}{ BICYCLIST STUDIES } \\
\hline Kim et al. (2007) & & $\uparrow$ & & \\
\hline $\begin{array}{c}\text { Islam and } \\
\text { Hossain (2015) }\end{array}$ & & $\uparrow$ & $\uparrow$ & \\
\hline McIntyre (2016) & $\downarrow$ & & & \\
\hline Eluru et al. (2008) & & $\uparrow$ & & $\uparrow$ \\
\hline
\end{tabular}

\subsection{Traffic conditions}

The impact of traffic volume and speed was also statistically associated with nonmotorized crashes and severity. Zheng (2014 ) suggested that as speed increased, the 
severity of the crash increased as well. The study revealed that severity increases more rapidly if the impact speed goes from medium speed $(25-50 \mathrm{mph})$ to high speed (>50 mph) rather than from low speed (<25 mph) to medium speed.

Kim et al. (2007) found that the likelihood of bicyclist fatalities and severe crashes increased with increases in the speed before the impact. As the impact increased beyond $20 \mathrm{mph}$, the probability of a severe or fatal crash increased substantially (Kim, Kim, G., \& Porello, 2007).

In terms of traffic volume, Halem et al. (2015) found that an increase of AADT at signalized intersections significantly increased the probability of severe injuries.

Allen-Munley et al. (2004) developed an urban bicycle route safety rating model based on crash severity and risk factors. The authors developed a new injury index created from detailed New Jersey police crash reports, for the period 1997 to 2000 . An ordinal logistic model was used to estimate the strength of the predictors on severity levels. The findings suggested that as AADT increased, severity decreased. This result is explained by another potential underlying effect, speed. When traffic volumes are high, the overall traffic speed is low, resulting in less severe crashes.

TABLE 6: Summary of traffic conditions review

\begin{tabular}{c|c|c}
\hline \hline & PEDESTRIAN STUDIES \\
\hline Author (year) & Traffic volume & Speed \\
\hline Zhao et al. (2014) & $\uparrow$ & $\uparrow$ \\
\hline Zheng (2014) & $\uparrow$ & $\uparrow$ \\
\hline Halem et al. (2015) & $\uparrow$ & \\
\hline Christie (1995) & $\uparrow$ & \\
\hline Oh et al. (2005) & $\uparrow$ & \\
\hline Haleem et al. (2015) & $\uparrow$ & \\
\hline Allen-Munley et al. (2004) & $\uparrow$ & \\
\hline Li et al. (2016) & $\uparrow$ & \\
\hline Aziz et al. (2013) & \\
\hline \hline
\end{tabular}


TABLE 6: Summary of traffic conditions review, continued

\begin{tabular}{c|c|c}
\hline \hline & BICYCLIST STUDIES \\
\hline Author (year) & Traffic volume & Speed \\
\hline Kim et al. (2007) & & $\uparrow$ \\
\hline Allen-Munley et al. (2004) & $\downarrow$ & \\
\hline \hline
\end{tabular}

\subsection{Road characteristics}

\section{- Number of lanes}

Zheng (2014) used crash data (4,126 observations) from the Florida DOT to understand how different road and traffic characteristics were useful to predict crash severity. The authors used an ordered probit model to assess their hypothesis. Severity was defined according to the $\mathrm{KABCO}$ scale. The authors found that when a crash occurred in a four lane or six lane road, it was more likely to be severe or fatal than one occurring in a two lane road.

Abdul-Aziz et al. (2013) found that the number of lanes was a statistical significant predictor for severity level of crashes involving vehicles and pedestrians as well. Crashes on single lane roads in New York neighborhoods were less severe than crashes on multilane roads.

\section{- Road width}

Allen-Munley et al. (2004) found that wider streets resulted in more severe bicyclist crashes. This road typology was associated with higher operating vehicle speeds, which can cause dangerous interactions with the bicyclists.

\section{- Posted speed limit}

Eluru et al. (2008) developed an ordered logit model to understand the effect of explanatory variables on severity levels for non-motorized transportation (pedestrians and 
bicyclists). Furthermore, an elasticity study was conducted to evaluate impact of the predictors on the probability of each level of severity. Data was collected from the general estimates system obtained from the National Highway Traffic Safety Administration. The authors classified speed limit as a set of dummy variables $(<25 \mathrm{mph}$, 25-50 mph, >50 mph) to understand its effect on non-motorized crash severity. Crashes tended to be more severe at roads with high posted speed limits. Zahabi et al. (2011) found that posted speed limit was not a statistically significant variable to explain bicyclist crash severity. The authors explained that drivers did not follow posted speed limits, making road geometry a better predictor. McIntyre (2016) also found that posted speed limit was only significant to predict pedestrian crash severity but not bicyclist crash severity. Pedestrian crashes that occurred on roads with a posted speed limit above 25 mph tended to be more severe and fatal than on roads with a lower speed limit.

\section{- Road classification}

Findings suggested that pedestrian and bicyclist severity levels increased with arterials and freeways designated roads. Crashes at arterials were more dangerous than crashes at local roads (Zahabi, Strauss, \& Miranda-Moreno, 2011). On another study, the authors found that bicyclists riding on roadways designated for high AADT and speeds (such as highways), were more likely to be severely injured if a crash occurred (Allen-Munley, Daniel, \& Dhar, 2004).

McIntyre (2016) explored the pedestrian and bicyclist severity determinants in San Francisco. Built environment variables such as crosswalks and bicycle facilities were considered in the analysis. Severity was classified into four categories: complaint of pain, other visible injury, severe and fatal. The study used a logistic regression model to 
determine the likelihood of a bicyclist or pedestrian severity level. The findings suggested that crashes on major roads (highways) tended to be more severe than on other roads.

\section{- Road surface}

Abdul-Aziz et al. (2013) used pedestrian crash data collected from New York City (20022006). A total of 4,666 observations were considered. The study classified severity in three categories: fatal, severe injury and property damage and injury. For a few neighborhoods in New York, a wet surface was strongly associated with severe crashes (for the rest of the neighborhoods the findings were not significant). On the other hand, Halem et al. (2015) found that dry surfaces increased the likelihood of severe and fatal crashes possibly due to the increase on speeding and risky maneuvers.

\section{- Rural location}

Even if there were more crashes in urban areas, crashes in cities are less likely to be fatal or severe than in rural areas. This finding can be attributed to the low speed limits (Abdul-Aziz, Ukkusuri, \& Hasan, 2013).

\section{- Intersections}

Zahabi et al. found that pedestrian crashes at intersections were less severe than crashes at other sections of the roads. The finding was attributed to the low vehicle speeds at these locations. Islam and Hossain (2015) also found that crashes at intersections were found to increase the probability of non-injury crashes, for both pedestrians and bicyclists. The authors explained that users on the road were more cautious before making a decision. 


\section{- Signalized intersections}

Abdul-Aziz et al. (2013) suggested that crashes are less likely to be fatal at unsignalized intersections since drivers and pedestrians were more cautious while crossing. Correspondingly, Zahabi et al. (2011) found that crashes at signalized intersections tended to be more dangerous for bicyclists due to the high number of conflicts with drivers. Sze and Wong (2007) found similar results.

\section{- Crosswalk}

Haleem et al. (2015) assessed pedestrian crash severity levels at signalized and nonsignalized locations. The authors analyzed a total of 4,923 pedestrian crashes in state roads in Florida. Data was verified and completed based on police reports and sketches. Severity was defined based on the KABCO scale. The findings suggested that standard crosswalks at unsignalized intersections were associated with $1.36 \%$ reduction in pedestrian severe injuries (Haleem, Alluri, \& Gan, 2015). On the other hand, Sze and Wong (2007) findings suggested that crashes that occurred at a crosswalk or within $15 \mathrm{~m}$ of it led to higher risk of mortality and severe injury compared to pedestrians walking on the sidewalk. This result was intuitive, even if pedestrians were somehow protected in crosswalks; they interacted with vehicles, increasing the risk of a crash. Similarly, Tay et al. (2011) found that crosswalks were conflict points of pedestrians and vehicle interactions, resulting in a higher probability (compared to other road segments) of pedestrians getting severely injured. 
TABLE 7: Summary of road characteristics review

\begin{tabular}{|c|c|c|c|c|c|c|c|c|c|}
\hline \multicolumn{10}{|c|}{ PEDESTRIAN STUDIES } \\
\hline $\begin{array}{c}\text { Author } \\
\text { (year) }\end{array}$ & $\begin{array}{l}\text { Major road } \\
\text { classification }\end{array}$ & $\begin{array}{c}\text { Presence } \\
\text { of a } \\
\text { crosswalk }\end{array}$ & $\begin{array}{c}\text { Rural } \\
\text { location }\end{array}$ & $\begin{array}{l}\text { Road } \\
\text { width }\end{array}$ & $\begin{array}{c}\text { Number } \\
\text { of lanes }\end{array}$ & $\begin{array}{c}\text { Wet } \\
\text { road } \\
\text { surface }\end{array}$ & $\begin{array}{c}\text { Posted } \\
\text { speed } \\
\text { limit }\end{array}$ & $\begin{array}{c}\text { Intersection } \\
\text { location }\end{array}$ & $\begin{array}{l}\text { Signalized } \\
\text { intersection }\end{array}$ \\
\hline $\begin{array}{c}\text { Tay et al. } \\
\text { (2011) }\end{array}$ & $\uparrow$ & $\uparrow$ & $\uparrow$ & $\uparrow$ & & & & $\uparrow$ & \\
\hline $\begin{array}{l}\text { Zheng } \\
(2014) \\
\end{array}$ & & & & & $\uparrow$ & & & & \\
\hline $\begin{array}{l}\text { Clifton et } \\
\text { al. (2007) } \\
\end{array}$ & & $\downarrow$ & & & & & & & \\
\hline $\begin{array}{c}\text { Seyed et } \\
\text { al. }(2011)\end{array}$ & $\uparrow$ & & & & & & & $\downarrow$ & \\
\hline $\begin{array}{l}\text { Torbic et } \\
\text { al. (2010) }\end{array}$ & & & & & & & & & $\uparrow$ \\
\hline $\begin{array}{c}\text { Tarko and } \\
\text { Azam } \\
(2011)\end{array}$ & & & $\uparrow$ & & & & $\uparrow$ & & \\
\hline $\begin{array}{l}\text { Haleem et } \\
\text { al. }(2015)\end{array}$ & & $\downarrow$ & & & & - & & & \\
\hline $\begin{array}{l}\text { Li et al. } \\
\text { (2016) }\end{array}$ & & & & & & & $\uparrow$ & & \\
\hline $\begin{array}{l}\text { Eluru et } \\
\text { al. (2008) }\end{array}$ & & & & & & & $\uparrow$ & & $\uparrow$ \\
\hline $\begin{array}{c}\text { Sze and } \\
\text { Wong } \\
(2007)\end{array}$ & & $\uparrow$ & & & & & $\uparrow$ & & $\uparrow$ \\
\hline $\begin{array}{c}\text { Beck et al. } \\
(2016)\end{array}$ & $\uparrow$ & & & & & & & & \\
\hline
\end{tabular}

$\infty$ 
TABLE 7: Summary of road characteristics review, continued

\begin{tabular}{|c|c|c|c|c|c|c|c|c|c|}
\hline \multicolumn{10}{|c|}{ PEDESTRIAN STUDIES } \\
\hline $\begin{array}{l}\text { Author } \\
\text { (year) }\end{array}$ & $\begin{array}{l}\text { Major road } \\
\text { classification }\end{array}$ & $\begin{array}{c}\text { Presence } \\
\text { of a } \\
\text { crosswalk } \\
\end{array}$ & $\begin{array}{c}\text { Rural } \\
\text { location }\end{array}$ & $\begin{array}{l}\text { Road } \\
\text { width }\end{array}$ & $\begin{array}{l}\text { Number } \\
\text { of lanes }\end{array}$ & $\begin{array}{c}\text { Wet } \\
\text { road } \\
\text { surface }\end{array}$ & $\begin{array}{l}\text { Posted } \\
\text { speed } \\
\text { limit } \\
\end{array}$ & $\begin{array}{c}\text { Intersection } \\
\text { location }\end{array}$ & $\begin{array}{l}\text { Signalized } \\
\text { intersection }\end{array}$ \\
\hline $\begin{array}{l}\text { Islam and } \\
\text { Hossain } \\
(2015) \\
\end{array}$ & $\uparrow$ & & $\uparrow$ & & & & & $\downarrow$ & \\
\hline $\begin{array}{c}\text { McIntyre } \\
(2016)\end{array}$ & $\uparrow$ & $\downarrow$ & & & & & $\uparrow$ & & \\
\hline $\begin{array}{c}\text { Sasidharan } \\
\text { et al. } \\
(2015) \\
\end{array}$ & $\uparrow$ & $\downarrow$ & & & & & $\uparrow$ & & \\
\hline $\begin{array}{l}\text { Zahabi et } \\
\text { al. (2011) }\end{array}$ & $\bar{\uparrow}$ & & & & & & & $\downarrow$ & \\
\hline $\begin{array}{l}\text { Abdul- } \\
\text { Aziz et al. } \\
(2013)\end{array}$ & & & $\uparrow$ & & $\uparrow$ & $\uparrow$ & $\uparrow$ & & $\uparrow$ \\
\hline \multicolumn{10}{|c|}{ BICYCLIST STUDIES } \\
\hline $\begin{array}{l}\text { Zahabi et } \\
\text { al. (2011) }\end{array}$ & $\uparrow$ & $\uparrow$ & & & & & - & $\uparrow$ & $\uparrow$ \\
\hline $\begin{array}{l}\text { Islam and } \\
\text { Hossain } \\
(2015) \\
\end{array}$ & $\uparrow$ & & $\uparrow$ & & & & & $\downarrow$ & \\
\hline $\begin{array}{c}\text { Eluru et al. } \\
(2008)\end{array}$ & & & & & & & $\bar{\uparrow}$ & & \\
\hline $\begin{array}{c}\text { McIntyre } \\
(2016)\end{array}$ & $\uparrow$ & & & & & & - & & \\
\hline $\begin{array}{c}\text { Allen- } \\
\text { Munley et } \\
\text { al. } \\
(2004)\end{array}$ & $\uparrow$ & & & $\uparrow$ & & & & & \\
\hline
\end{tabular}

$\varpi$ 


\subsection{Studies in Oregon}

The ODOT Pedestrian and Bicycle Safety Implementation Plan (Kittelson \& Associates, Inc. 2014) was developed to identify the most unsafe locations along corridors in Oregon with the highest potential for reducing frequency and severity of pedestrian and bicycle crashes.

Pedestrian and Bicyclist crash data (frequency and severity) for the period 2007 to 2011 were collected. Data on pedestrian volume was not available for the state of Oregon, so vehicle traffic volume was used as a surrogate of pedestrian exposure to traffic. This section presents the main findings of the ODOT Pedestrian and Bicycle Safety Implementation Plan.

The results from this report reinforced the findings from the literature review. Pedestrian, bicyclist, and driver behavior characteristics, along with road and crosswalk features, had a strong association with pedestrian crash frequency. The authors revealed that time of the year, lighting conditions, midblock crossing, driver behavior, and alcohol / drug involvement were the main factors associated with pedestrians crashes. For bicyclist crashes, the authors found that most of the crashes occurred at intersections, caused by drivers turning right. 
TABLE 8: ODOT Pedestrian and Bicycle Safety Implementation Plan findings

\begin{tabular}{|c|c|c|}
\hline & PEDESTRIAN & BICYCLIST \\
\hline $\begin{array}{c}\text { Individual } \\
\text { characteristics }\end{array}$ & $\begin{array}{l}\text { The majority of impaired cases (by alcohol } \\
\text { or drug involvement) involved an impaired } \\
\text { pedestrian rather than an impaired driver. } \\
\text { The most common reported pedestrian error } \\
\text { was jaywalking. } \\
\text { At intersections, the most frequent crash } \\
\text { cause involved vehicles going straight and } \\
\text { jaywalking. }\end{array}$ & - Not available in the report \\
\hline $\begin{array}{c}\text { Temporal } \\
\text { characteristics }\end{array}$ & $\begin{array}{l}\text { The number of reported pedestrian crashes } \\
\text { (from the ODOT Crash Analysis Reporting } \\
\text { unit) increased between October and March. } \\
\text { Poor lighting conditions (with and without } \\
\text { streetlights) was a factor in pedestrian crash } \\
\text { frequency and severity. }\end{array}$ & - Not available in the report \\
\hline $\begin{array}{c}\text { Road } \\
\text { characteristics }\end{array}$ & $\begin{array}{l}\text { - } 80 \% \text { of crashes in urban areas occurred in } \\
\text { dark conditions. } \\
\text { At controlled intersections, left-turn crashes } \\
\text { were more frequent because drivers failed to } \\
\text { yield to pedestrians. However, the severity } \\
\text { of "left hook" crashes is known to be less } \\
\text { severe than collisions with pedestrians when } \\
\text { the car is travelling straight. Controlled } \\
\text { intersections with permitted or } \\
\text { protected/permitted left-turn phases were } \\
\text { found to be a potential crash risk factor for } \\
\text { pedestrians. } \\
\text { In rural areas, a low percentage of crashes } \\
\text { involving vehicles making right turns and } \\
\text { failing to yield to pedestrians were severe at } \\
\text { both controlled and uncontrolled } \\
\text { intersections. } \\
\text { In urban areas, the majority of crashes } \\
\text { occurred on arterials or collectors. } \\
\text { Additionally, the majority of severe crashes } \\
\text { occurred on roadways with four lanes. } \\
\text { In urban areas, } 45 \% \text { of crashes occurred in } \\
\text { locations with speed limits of } 45 \text { mph or } \\
\text { higher. } \\
\text { Most of the crashes in rural areas ( } 80 \% \text { ) } \\
\text { occurred on roadways with speed limits } \\
\text { above } 45 \text { mph. } \\
\text { The majority of crashes occurred in urban } \\
\text { areas (for highways across Oregon, the } \\
\text { proportion was } 80 \% \text { ) (FIGURE } 1 \text { a) }\end{array}$ & $\begin{array}{l}\text { Most of the crashes in } \\
\text { Oregon Highways and the } \\
\text { Portland Metro Area } \\
\text { occurred at intersections. } \\
\text { In Oregon Highways, 63\% } \\
\text { of the crashes occurred on } \\
\text { non-signalized intersections, } \\
\text { while only 57\% occurred at } \\
\text { these locations in Portland } \\
\text { intersections. } \\
\text { Half of severe crashes at } \\
\text { signalized intersections } \\
\text { occurred less than 100' from } \\
\text { a transit stop } \\
\text { Risk factors found from } \\
\text { crash pattern analysis were } \\
\text { driveway density, undivided } \\
\text { 4-lane roadways, lack of } \\
\text { bicycle facilities, presence of } \\
\text { a traffic signal, AADT and } \\
\text { posted speed limit. } \\
\text { Almost } 90 \% \text { of the crashes } \\
\text { occurred at urban areas. }\end{array}$ \\
\hline
\end{tabular}


TABLE 8: ODOT Pedestrian and Bicycle Safety Implementation Plan findings, continued

\begin{tabular}{|c|c|c|}
\hline & PEDESTRIAN & BICYCLIST \\
\hline $\begin{array}{c}\text { Crosswalk } \\
\text { characteristics }\end{array}$ & $\begin{array}{l}\text { - The majority of pedestrian crashes that } \\
\text { occurred away from intersections involved } \\
\text { pedestrians attempting to cross at midblock } \\
\text { locations and drivers failing to yield. } \\
\text { The proportion of crashes that occurred in } \\
\text { locations without sidewalks is } 45 \% \text { and } \\
80 \% \text { in urban and rural areas, respectively. } \\
\text { In the Portland Metro Area, the majority of } \\
\text { the crashes occurred at intersections (61\%). } \\
\text { At highways across Oregon, the proportion } \\
\text { was the same for intersections and } \\
\text { segments. Crashes were more frequent at } \\
\text { controlled intersections (62\%) in the } \\
\text { Portland Metro Area compared to } \\
\text { uncontrolled intersections. Along highways } \\
\text { across Oregon, the proportion was higher at } \\
\text { uncontrolled intersections (52\%). } \\
\text { FIGURE } 2 \text { (a) }\end{array}$ & - $\quad$ Not Applicable \\
\hline $\begin{array}{c}\text { Crash } \\
\text { characteristics }\end{array}$ & $\begin{array}{l}\text { In most of the severe crashes, the driver } \\
\text { was going straight and failed to yield to } \\
\text { the pedestrian }\end{array}$ & 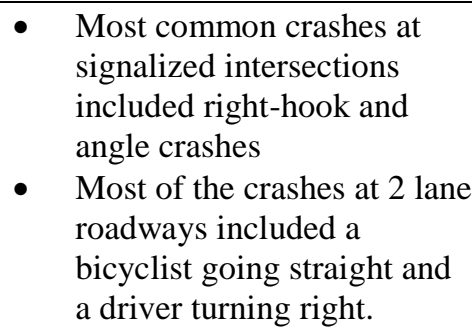 \\
\hline
\end{tabular}

The next figures (FIGURE 1 and FIGURE 2) show the distribution of pedestrian and bicyclist crashes in Oregon Highways and the Portland Metro Area. These figures are adapted based on the information available in the Kittelson \& Associates report (2014).

In FIGURE 1, observations are grouped by urban and rural areas. In FIGURE 2, observations are grouped by intersection and segments. 


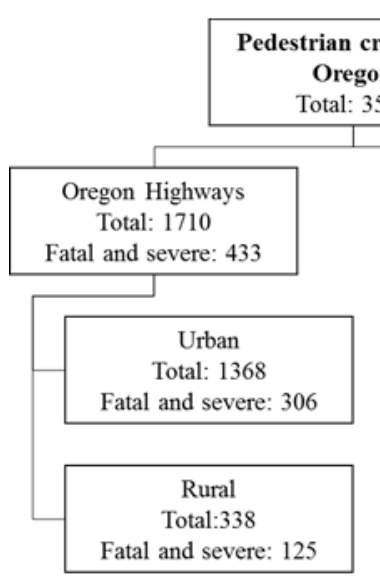

(a)

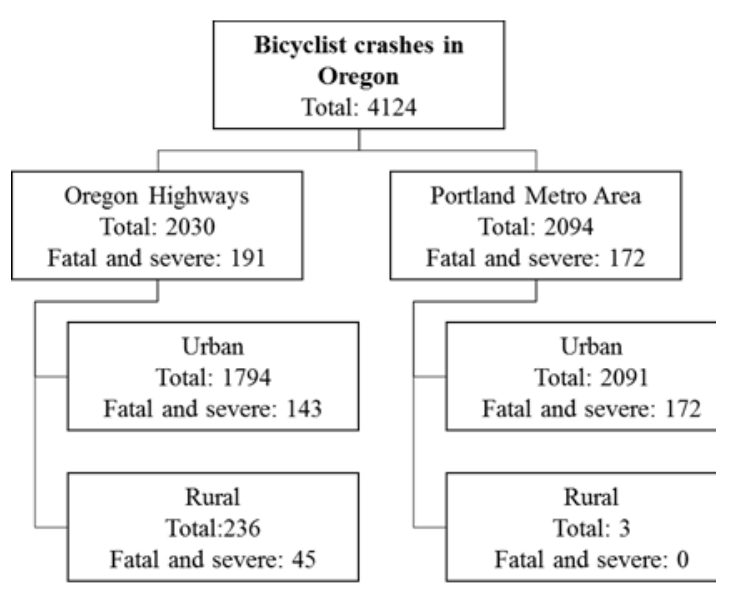

(b)

FIGURE 1: (a) Pedestrian and (b) Bicyclist crashes by location. This figure is adapted from Kittelson \& Associates, Inc. (2014)

For pedestrian and bicyclist crashes the distribution is similar. Most of the crashes occurred in urban areas, where most of the fatal and severe crashes are located as well. FIGURE 2 also highlights that there are almost 700 more bicyclist crashes than pedestrian crashes. The authors mentioned that one of the key problems in Oregon was the lack of bicycle infrastructure in some high vehicle speed corridors. 


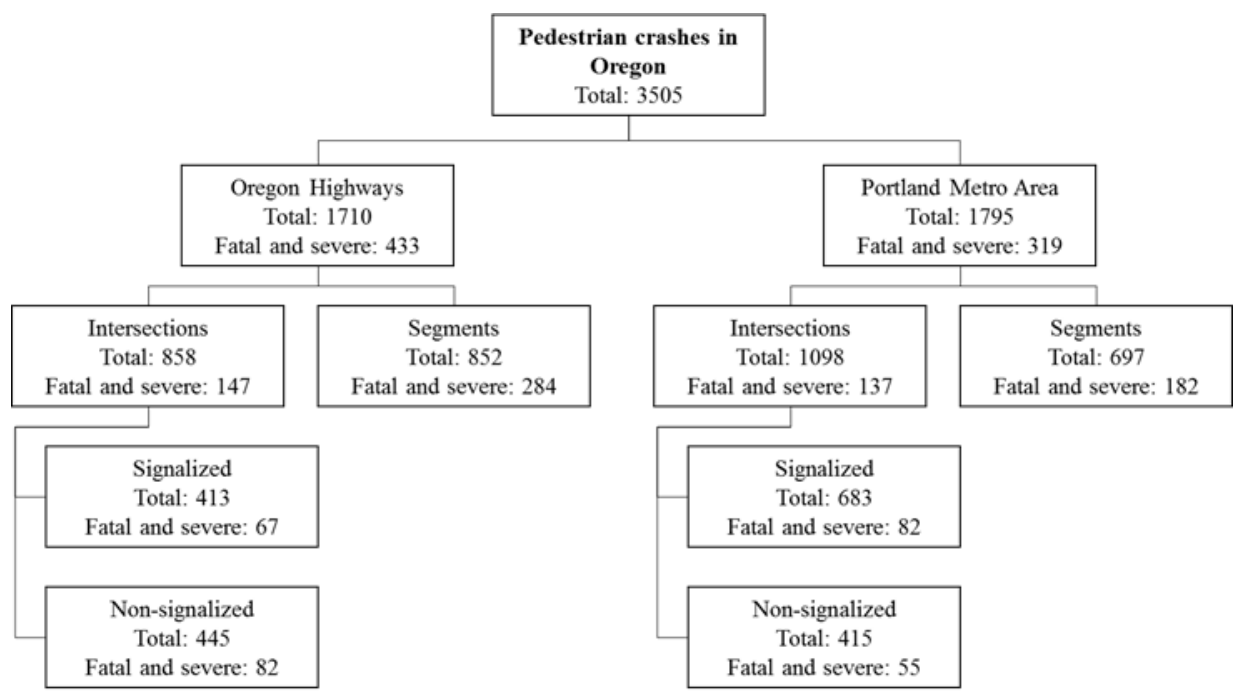

(a)

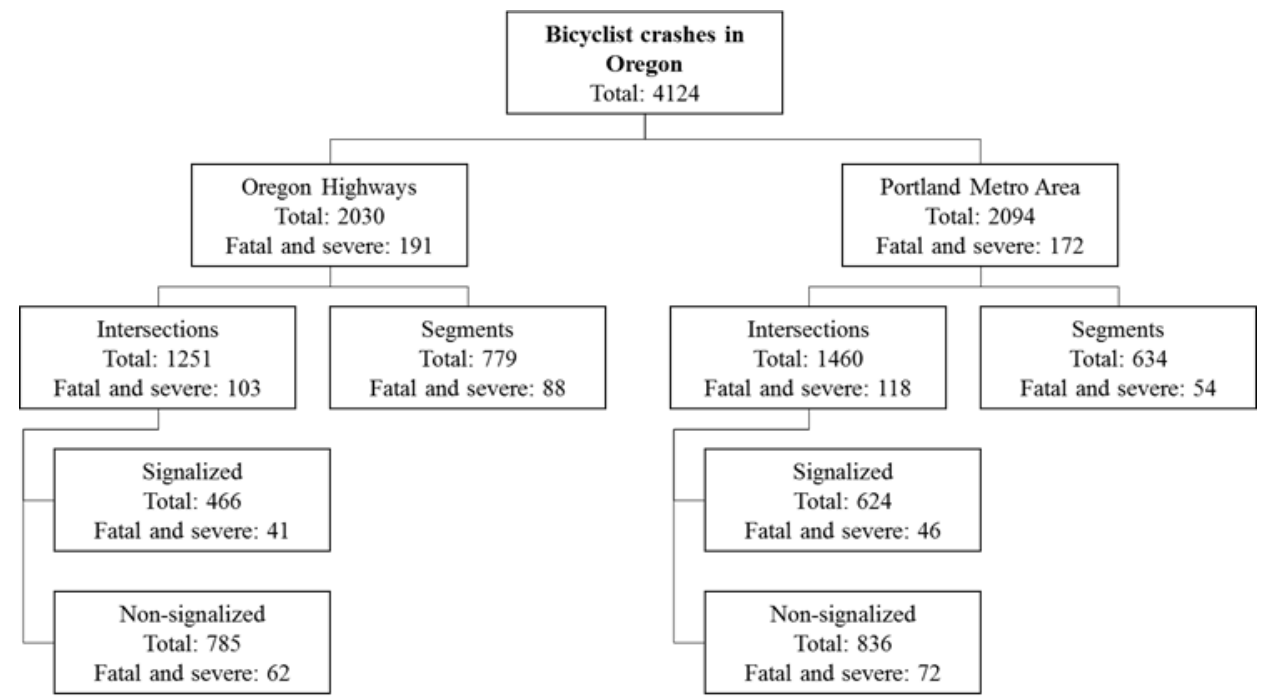

(b)

FIGURE 2: (a)Pedestrian and (b)Bicyclist crashes by road characteristics and intersection type. This figure is adapted from Kittelson \& Associates, Inc. (2014) 
Additionally, most of the crashes occurred on intersections. In The Portland Metro Area, most of the pedestrian crashes occurred at signalized intersections while most of bicyclist crashes occurred at non-signalized intersections.

\subsection{Summary}

Substantial research efforts have been made on analyzing crash severity when a pedestrian and/or bicyclist are involved. The findings of this review have helped us to start answering some of the research objective of this study: what are the main risk factors associated to pedestrian and bicyclist crash severity, and how are these levels affected by exposure. The review suggested that variables linked to the participant (driver, pedestrian, and bicyclist), road characteristics, environmental conditions, location characteristics, traffic conditions and crash characteristics are linked to severity. What is novel of approaching traffic safety in terms of severity and not crash frequency, is the fact that improvements and countermeasures can be implemented to protect vulnerable users and control for the randomness of some crash events.

Most of the studies reviewed (12 out of 12 for pedestrians, and 3 out of 4 for bicyclists) suggested that elder non-motorized travelers had a higher risk of being involved in a severe or fatal crash. These findings can be attributed to the fact that walking speed, visibility reaction time, and body resistance tended to be lower for this demographic group.

For gender, the trend was not as clear. Different studies (7) found contradictory results. The review of the literature did not conclude that men or women were involved in more severe or fatal crashes than the other gender. These findings were also different 
than the ones found in studies that analyze crash rates, where male pedestrians had a higher likelihood of being involved in a crash than female pedestrians.

In terms of road characteristics, the literature review suggested that a major roads (7 out of 7 studies), rural areas (4 out of 4 studies), an increase in road width ( 2 out of 2 studies) and number of lanes ( 2 out of 7 studies), and posted speed limit ( 7 out of 7 studies), increased the likelihood of severe or fatal pedestrian crashes. For bicyclists, the trend was similar, with the only difference in posted speed limit. Only 1 study out of 3 found a statistical relationship with posted speed limit.

Another interesting result was found in studies that analyzed crashes at intersections. Most of the studies ( 3 out of 4 ) found that the likelihood of a severe or fatal pedestrian crash at intersections was lower than that for segments. Furthermore, when the intersection was signalized, the probability of a severe or fatal crash increased (4 out of 4 studies).

Environmental conditions were found to be significant predictors of severity, for both pedestrians and bicyclists. Inclement weather, poor light time conditions (or nighttime), peak hours, and weekend days increased the likelihood of severe and fatal crashes. In terms of exposure, we reviewed studies that explored land uses as a surrogate of pedestrian and bicyclist exposure. Researchers used land use to examine if areas with high pedestrian and bicyclist activity resulted in more severe crashes than other land use typologies. Commercial land use and big generators of pedestrian volumes were found to increase the probability of severe crashes for pedestrians. Furthermore, some studies found that land use designated as industrial is associated with less severe and fatal pedestrian crashes due to the low levels of pedestrian activity in these zones. Two studies 
found that when a school is present near the crash event, the likelihood of severe or fatal crash decreases. Schools tended to have better traffic management to reduce speeds, which protects vulnerable users.

The literature review revealed that as traffic volume and speed increase, the probability of a severe or fatal crash with a non-motorized participant increased. One study found that bicyclists were safer (in terms of severity) with higher volumes since speed tended to decrease under this condition. Moreover, 15 out of 15 studies have reported that an increase in vehicle size and weight increase the probability of a severe or fatal crash with a pedestrian or bicyclist involved.

A few studies have assessed the factors that may contribute to bicyclist crash severity. Finally, all of the studies reviewed showed that when a crash is caused by an alcohol intoxicated driver, the likelihood of a fatal or severe crash increased.

We also identified a gap in the literature in terms of the relationship between pedestrian and bicyclist crash severity. Countermeasures that were proposed to only one non-motorized travel mode may have a negative effect on likelihood of severity for the other mode.

TABLE 9: Literature review summary

\begin{tabular}{|c|c|c|c|}
\hline VARIABLE & DESCRIPTION & CATEGORY & $\begin{array}{c}\text { Increase }(\uparrow) \text { or } \\
\text { decrease }(\downarrow) \text { of fatal } \\
\text { or severe crash } \\
\text { likelihood }\end{array}$ \\
\hline \multirow[t]{2}{*}{ Gender } & \multirow{2}{*}{$\begin{array}{l}\text { There is no statistically significant } \\
\text { difference between women and } \\
\text { men in terms of crash severity }\end{array}$} & Female & - \\
\hline & & Male & - \\
\hline \multirow[t]{3}{*}{ Age } & \multirow{3}{*}{$\begin{array}{l}\text { The likelihood of being involved in } \\
\text { crash with severity level K or A is } \\
\text { higher for seniors and children }\end{array}$} & Children & $\uparrow$ \\
\hline & & Adult & \\
\hline & & Senior & $\uparrow$ \\
\hline
\end{tabular}


TABLE 9: Literature review summary, continued

\begin{tabular}{|c|c|c|c|}
\hline VARIABLE & DESCRIPTION & CATEGORY & $\begin{array}{l}\text { Increase }(\uparrow) \text { or } \\
\text { decrease }(\downarrow) \text { of } \\
\text { fatal or severe } \\
\text { crash likelihood } \\
\end{array}$ \\
\hline $\begin{array}{l}\text { Posted Speed } \\
\text { Limit }\end{array}$ & $\begin{array}{l}\text { As posted speed limit increases, } \\
\text { crash severity increases }\end{array}$ & & $\uparrow$ \\
\hline \multirow{2}{*}{$\begin{array}{l}\text { Streetlight } \\
\text { condition }\end{array}$} & \multirow{2}{*}{$\begin{array}{l}\text { No street lighting increases the } \\
\text { likelihood of a fatal or severe } \\
\text { injured crash. }\end{array}$} & Present & - \\
\hline & & Not present & $\uparrow$ \\
\hline \multirow[t]{2}{*}{ Road surface } & \multirow{2}{*}{$\begin{array}{l}\text { Wet road surface increases the } \\
\text { likelihood of a fatal or severe } \\
\text { injured crash. }\end{array}$} & Wet & $\uparrow$ \\
\hline & & Dry & - \\
\hline Road Width & $\begin{array}{c}\text { As road width increases, crash } \\
\text { severity increases }\end{array}$ & & $\uparrow$ \\
\hline \multirow[t]{2}{*}{ Crosswalk } & \multirow{2}{*}{$\begin{array}{c}\text { The presence of a crosswalk } \\
\text { decreases the likelihood of a fatal } \\
\text { or severe injured crash. }\end{array}$} & Present & - \\
\hline & & Not present & $\bar{\uparrow}$ \\
\hline \multirow[t]{2}{*}{ Intersection } & \multirow{2}{*}{$\begin{array}{l}\text { Controlled intersections tend to } \\
\text { have less fatal or severe crashes } \\
\text { than uncontrolled intersections }\end{array}$} & Controlled & - \\
\hline & & Uncontrolled & $\uparrow$ \\
\hline \multirow{3}{*}{$\begin{array}{c}\text { Road } \\
\text { classification }\end{array}$} & \multirow{3}{*}{$\begin{array}{l}\text { Principal arterials tend to have } \\
\text { more fatal or severe crashes than } \\
\text { other functional classes }\end{array}$} & Arterial & $\uparrow$ \\
\hline & & Collector & - \\
\hline & & Local & - \\
\hline $\begin{array}{l}\text { Number of } \\
\text { lanes }\end{array}$ & $\begin{array}{c}\text { As the number of lanes increase, } \\
\text { crash severity increases }\end{array}$ & & $\uparrow$ \\
\hline \multirow[t]{3}{*}{ Weather } & \multirow{3}{*}{$\begin{array}{l}\text { Clear day conditions tend to have } \\
\text { less fatal and severe crashes than } \\
\text { other weather conditions (e.g. rain, } \\
\text { snow, fog) }\end{array}$} & Clear day & - \\
\hline & & Rainy day & $\uparrow$ \\
\hline & & Snowy day & $\uparrow$ \\
\hline $\begin{array}{l}\text { Light } \\
\text { conditions }\end{array}$ & $\begin{array}{l}\text { Dark conditions increase the } \\
\text { likelihood of a fatal or severe crash. }\end{array}$ & Dark & $\uparrow$ \\
\hline $\begin{array}{l}\text { Time of the } \\
\text { day }\end{array}$ & $\begin{array}{l}\text { Crashes that occurred at peak hours } \\
\text { are more likely to result in a fatality } \\
\text { or severe injured output. }\end{array}$ & Peak hour & $\bar{\uparrow}$ \\
\hline Transit access & $\begin{array}{l}\text { There is no statistically significant } \\
\text { difference between roads with and } \\
\text { without transit access (stop/station) } \\
\text { in terms of crash severity }\end{array}$ & & $\bar{\uparrow}$ \\
\hline \multirow[t]{3}{*}{ Land use } & \multirow{3}{*}{$\begin{array}{l}\text { Industrial land uses tend to have } \\
\text { more fatal or severe crashes }\end{array}$} & Residential & - \\
\hline & & Commercial & - \\
\hline & & Industrial & $\uparrow$ \\
\hline $\begin{array}{l}\text { Employment } \\
\text { density }\end{array}$ & $\begin{array}{c}\text { High employment density areas } \\
\text { tend to have less fatal or severe } \\
\text { crashes }\end{array}$ & & $\uparrow$ \\
\hline $\begin{array}{l}\text { Traffic } \\
\text { Volume }\end{array}$ & $\begin{array}{l}\text { As traffic volume increases, crash } \\
\text { severity increases }\end{array}$ & & $\uparrow$ \\
\hline
\end{tabular}


TABLE 9: Literature review summary, continued

\begin{tabular}{c|c|c|c}
\hline \hline VARIABLE & DESCRIPTION & CATEGORY & $\begin{array}{c}\text { Increase }(\uparrow) \text { or } \\
\text { decrease }(\downarrow) \text { of } \\
\text { fatal or severe } \\
\text { crash likelihood }\end{array}$ \\
\hline Vehicle size & $\begin{array}{c}\text { Fatal and severe crashes tend to } \\
\text { occur when a heavy vehicle is } \\
\text { involved in the crash }\end{array}$ & $\uparrow$ \\
\hline $\begin{array}{c}\text { Alcohol } \\
\text { intoxication }\end{array}$ & $\begin{array}{c}\text { Crashes caused by an alcohol } \\
\text { intoxicated driver increased the } \\
\text { odds of fatal or severe crashes }\end{array}$ & & $\uparrow$ \\
\hline \hline
\end{tabular}




\section{DATA SOURCES}

Pedestrian and bicyclist crash data for the years 2007 to 2014 was collected from the Oregon Statewide Crash Data System. The database consisted of fatal, bodily injury and damage to personal property (more than $\$ 1,500)$ traffic crashes. Crashes included those that occurred on city streets, county roads, and state highways. Only crashes that involved at least one motorized vehicle are reported in the database (e.g. the database does not contain information related to pedestrian-bicycle crashes or bicycle-bicycle crashes).

The database contained a total of 13,309 crashes, where 6,162 involved pedestrians and 7,147 involved people riding a bicycle. Information related to the crash event, the characteristics of the vehicle, and participants involved was available in the database. Information on the crash event is collected by a police officer if this involved a fatality or severe injury. In Oregon, drivers also must file an accident and insurance report form with the DMV if there was a fatality or severe injury, or if the damage to the vehicle was over $\$ 1,500$, or the damage to any other participant's property was over $\$ 1,500$. A significant number of crashes were underreported because not all the crashes met the thresholds to be reported, or the participants of the crash chose to not report. Studies (Washington, Haque, Oh, \& Lee, 2014) (Yamamoto, Hashiji, \& Shankar, 2008) have found that on average, around $50 \%$ of the property only damage crashes were reported in different cities. FIGURE 3 shows the path/process on how the crash event information is recorded in the Oregon Statewide Crash Data System. 
DMV matches common

crash reports. DMV

records data for driver

info and insurance.
ODOT codes crash into database

FIGURE 3: Crash event processing

Finally, the database reported crash severity based on the five point KABCO scale.

Severity level for each participant was recorded. At the crash level, severity was reported based on the most severe injury (e.g. if a crash involved two participants, one with A and the other with B injury, the crash severity is reported as A)

TABLE 10: KABCO severity scale

\begin{tabular}{|c|c|c|}
\hline $\begin{array}{l}\text { KABCO } \\
\text { SCALE }\end{array}$ & & DESCRIPTION \\
\hline $\mathrm{K}$ & Fatal & $\begin{array}{l}\text { Fatality information includes crashes that result in the death of a } \\
\text { driver or a non-motorist within } 30 \text { days of the crash. }\end{array}$ \\
\hline A & Incapacitated & $\begin{array}{l}\text { Injury of the participant prevents him/her from walking, driving } \\
\text { or normally continuing the activities he or she was capable of } \\
\text { performing before the injury occurred. }\end{array}$ \\
\hline $\mathrm{B}$ & Visible Injury & $\begin{array}{l}\text { Injury to the participant which is evident to observers at the } \\
\text { scene of the crash (e.g. bruises, cuts, lacerations, etc.) }\end{array}$ \\
\hline $\bar{C}$ & $\begin{array}{l}\text { Complaint of } \\
\text { Pain }\end{array}$ & $\begin{array}{c}\text { Participant claimed being injured, however the injury is not } \\
\text { evident to observers (e.g. momentary unconsciousness, } \\
\text { complaint of pain, nausea, etc.) }\end{array}$ \\
\hline $\mathrm{O}$ & None & There was no bodily harm to the participant. \\
\hline
\end{tabular}

Additional information on the Oregon State Highway System was collected from the Oregon Department of Transportation's TransGIS database. The database contained georeferenced information of the roadway characteristics (e.g. pavement condition, number of lanes, lane width, AADT, road classification), safety (state and non-state crashes), and other themes (e.g. drainage, freight routes, rail network, public transit, road network, STIP projects, environmental protection, etc.) 
To account for the land use characteristics, we used the neighborhood concepts defined by Currans et al. (2015). For the Oregon census blocks, the researchers categorized different land uses into a set of six neighborhood concepts (A to F). These concepts were defined based on activity density (number of residents and jobs per acre of unprotected land), employment entropy (distribution of retail, office, industrial, services, and entertainment jobs), and intersection density (the number of intersections in the road network per square miles). The classification scheme transitioned from dense, urban environments, A or B, towards rural, less dense environments, F. Crashes in ODOT's facilities only occurred in concepts D, E and F. For a better illustration of these concepts, FIGURE shows images representing the different neighborhood concepts.

TABLE 11 describes the land use characteristics used to define the different neighborhood concepts.
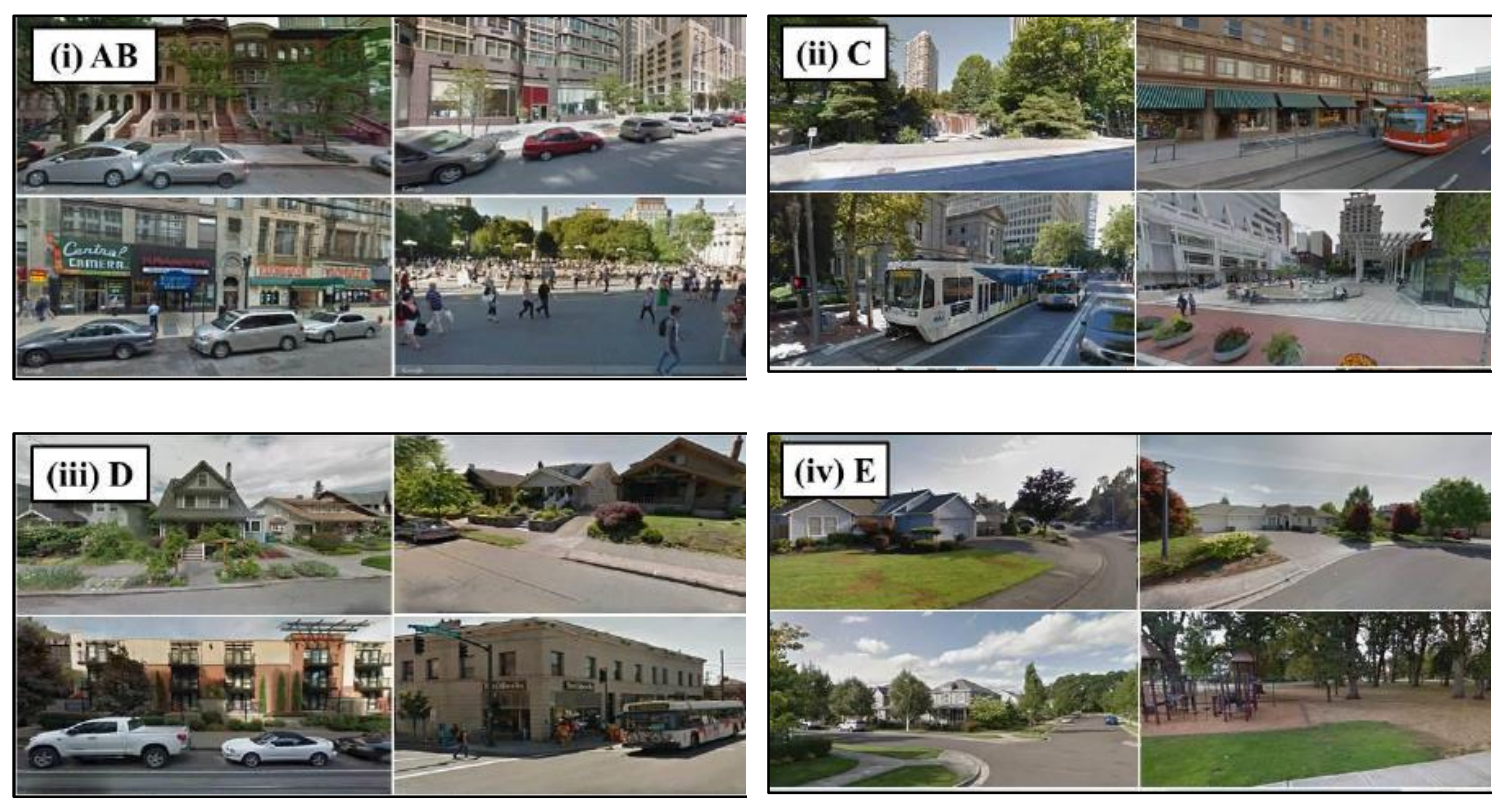

FIGURE 4: Neighborhood Concept Types, adapted from Currans et al. (2015) 


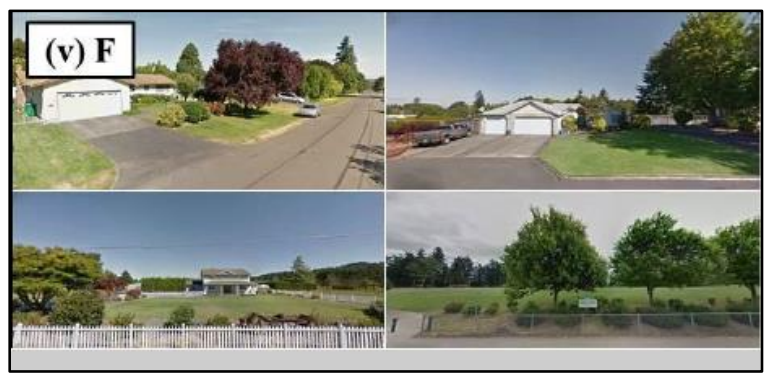

FIGURE 4: Neighborhood Concept Types, adapted from Currans et al. (2015), continued

TABLE 11: Neighborhood concept characteristics

\begin{tabular}{c|c|c|c}
\hline \hline $\begin{array}{c}\text { NEIGHBORHOOD } \\
\text { CONCEPT TYPE }\end{array}$ & $\begin{array}{c}\text { ACTIVITY } \\
\text { DENSITY } \\
\text { (RESIDENTS } \\
\text { AND JOBS PER } \\
\text { ACRE) }\end{array}$ & $\begin{array}{c}\text { EMPLOYMENT } \\
\text { ENTROPY } \\
\text { (UNITLESS) }\end{array}$ & $\begin{array}{c}\text { INTERSECTION } \\
\text { DENSITY } \\
\text { (INTERSECTIONS } \\
\text { PER SQUARE } \\
\text { MILE) }\end{array}$ \\
\hline A-B & 667 & 0.75 & 489 \\
\hline C & 245 & 0.75 & 189 \\
\hline D & 39 & 0.76 & 141 \\
\hline E & 20 & 0.67 & 73 \\
\hline \hline
\end{tabular}

As shown in TABLE 11, Neighborhoods with a concept of A and B have a higher

activity density and intersection density than the other concepts, while concept F,

represents a more suburban area, with low activity and intersection density and,

employment entropy. For Oregon, only four of the six neighborhood concepts exist, C, D,

E and F. 

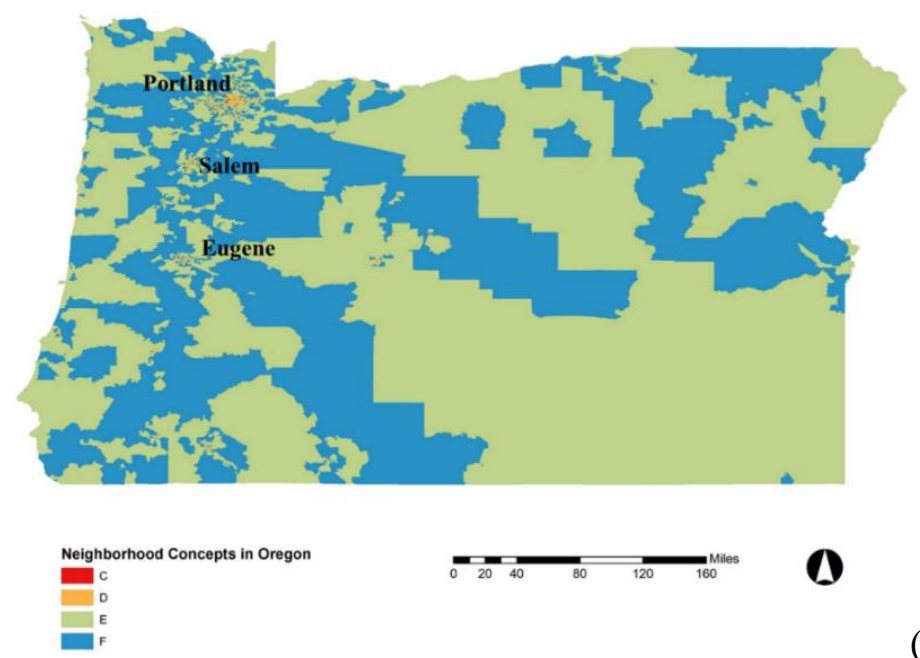

(a)

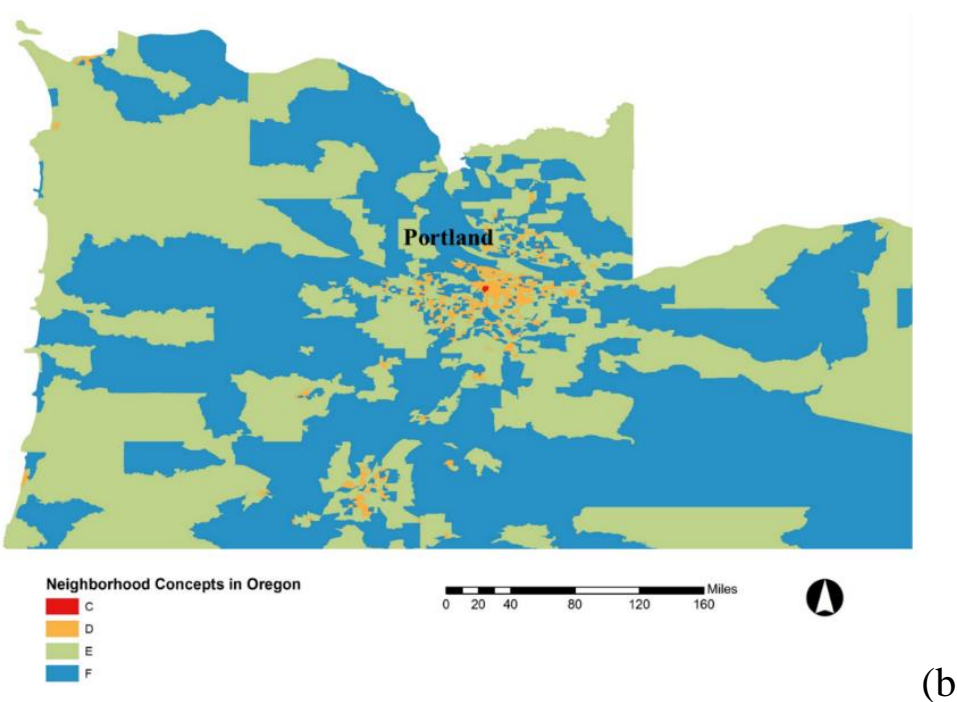

FIGURE 5: Oregon Neighborhood Concept, (a) State of Oregon, (b) Portland Metro Area 


\section{DESCRIPTIVE ANALYSIS}

This descriptive analysis is intended to present the data and uncover patterns in terms of risk factors for crash severity and neighborhood concepts. This first approximation to the data includes all the crashes that occurred in Oregon. Section 6 and 7 will only focus on crashes that occurred in the Oregon Highway System. Crash data from the Oregon Statewide Crash Data System was grouped by intersection and segment based on the traffic control device information available at the crash level. Crashes at driveways, curves, and bridge structures were not taken into account due to the few observations available. Crash information is presented as the number of crashes reported. Furthermore, crashes were grouped by neighborhood concept (Currans, Gherke, \& Clifton, 2015) to account for the differences in built environment in the likelihood of a crash occurrence.

\subsection{General Trends}

A total of 3,629 pedestrian crashes occurred at intersections from 2007 to 2011.

Additionally, 1,822 pedestrian crashes occurred on road segments. The majority of the crashes occurred in neighborhood concept D, followed by E, F and C. As seen in Figure 5, the urban metropolitan areas in Oregon were mainly defined as concept D and E, which explains why fewer pedestrian crashes occurred in concept C (188 pedestrian crashes).

In terms of crash severity, the findings suggested that injury level B had the largest number of crashes at intersections and segments. For areas with neighborhood concept $\mathrm{C}$, there has been an increase of these crashes during the last decade. 
By comparing crashes at segments and intersections across the years, one result stood out. There were more fatal (K) and incapacitated (A) crashes on road segments than at intersections. On segments of the road, vehicles tended to drive faster.

TABLE 12: Statewide pedestrian crashes by neighborhood concepts and year

\begin{tabular}{c|c|c|c|c|c|c|c|c|c|c}
\hline \hline \multirow{2}{*}{ Year } & \multicolumn{10}{c}{ Neighborhood Concept C } \\
& \multicolumn{1}{|c}{ Intersection (\%) } & \multicolumn{7}{c}{ Segment (\%) } \\
\cline { 2 - 12 } & K & $\mathbf{A}$ & $\mathbf{B}$ & $\mathbf{C}$ & $\mathbf{O}$ & $\mathbf{K}$ & $\mathbf{A}$ & $\mathbf{B}$ & $\mathbf{C}$ & $\mathbf{O}$ \\
\hline 2007 & 0 & 14 & 4 & 7 & 0 & 0 & 0 & 0 & 0 & 0 \\
\hline 2008 & 0 & 21 & 6 & 15 & 0 & 0 & 8 & 11 & 0 & 0 \\
\hline 2009 & 0 & 0 & 19 & 4 & 0 & 0 & 15 & 11 & 0 & 0 \\
\hline 2010 & 0 & 14 & 16 & 16 & 0 & 0 & 31 & 11 & 0 & 0 \\
\hline 2011 & 0 & 0 & 15 & 19 & 0 & 100 & 8 & 22 & 0 & 0 \\
\hline 2012 & 0 & 21 & 12 & 22 & 0 & 0 & 15 & 22 & 0 & 0 \\
\hline 2013 & 100 & 7 & 11 & 10 & 100 & 0 & 0 & 11 & 0 & 0 \\
\hline 2014 & 0 & 21 & 17 & 6 & 0 & 0 & 23 & 11 & 0 & 0 \\
\hline Total & 1 & 14 & 81 & 68 & 1 & 1 & 13 & 9 & 0 & 0 \\
\hline \hline
\end{tabular}

\begin{tabular}{c|c|c|c|c|c|c|c|c|c|c}
\hline \hline & \multicolumn{10}{c}{ Neighborhood Concept D } \\
\hline \multirow{2}{*}{ Year } & \multicolumn{1}{|c}{ Intersection (\%) } & \multicolumn{7}{c}{ Segment (\%) } \\
\cline { 2 - 12 } & K & A & B & C & O & K & A & B & C & O \\
\hline 2007 & 7 & 9 & 9 & 8 & 19 & 15 & 15 & 11 & 13 & 0 \\
\hline 2008 & 19 & 11 & 9 & 10 & 6 & 9 & 14 & 10 & 16 & 20 \\
\hline 2009 & 11 & 11 & 11 & 9 & 19 & 9 & 8 & 10 & 13 & 20 \\
\hline 2010 & 4 & 8 & 14 & 14 & 0 & 11 & 17 & 16 & 12 & 0 \\
\hline 2011 & 6 & 15 & 13 & 16 & 6 & 17 & 11 & 12 & 16 & 20 \\
\hline 2012 & 17 & 14 & 12 & 15 & 13 & 13 & 15 & 15 & 14 & 0 \\
\hline 2013 & 15 & 14 & 15 & 13 & 25 & 15 & 13 & 13 & 9 & 20 \\
\hline 2014 & 22 & 19 & 18 & 15 & 13 & 11 & 7 & 13 & 8 & 20 \\
\hline Total & 54 & 173 & 852 & 638 & 16 & 46 & 111 & 298 & 160 & 5 \\
\hline \hline
\end{tabular}


TABLE 12: Statewide pedestrian crashes by neighborhood concepts and year, continued

\begin{tabular}{c|c|c|c|c|c|c|c|c|c|c}
\hline \hline & \multicolumn{10}{c}{ Neighborhood Concept E } \\
\hline \multirow{2}{*}{ Year } & \multicolumn{1}{|c|}{ Intersection (\%) } & \multicolumn{7}{c}{ Segment (\%) } \\
\cline { 2 - 12 } & K & A & B & C & O & K & A & B & C & O \\
\hline 2007 & 17 & 10 & 9 & 5 & 6 & 10 & 13 & 11 & 10 & 10 \\
\hline 2008 & 8 & 11 & 9 & 7 & 0 & 14 & 13 & 9 & 13 & 10 \\
\hline 2009 & 8 & 9 & 10 & 11 & 25 & 7 & 15 & 12 & 19 & 20 \\
\hline 2010 & 10 & 16 & 12 & 11 & 6 & 20 & 11 & 13 & 11 & 10 \\
\hline 2011 & 17 & 15 & 14 & 13 & 6 & 9 & 16 & 14 & 14 & 30 \\
\hline 2012 & 19 & 12 & 17 & 17 & 6 & 16 & 12 & 16 & 12 & 0 \\
\hline 2013 & 6 & 15 & 14 & 17 & 38 & 13 & 10 & 15 & 8 & 20 \\
\hline 2014 & 15 & 12 & 16 & 18 & 13 & 12 & 10 & 10 & 12 & 0 \\
\hline Total & 48 & 146 & 588 & 471 & 16 & 117 & 148 & 345 & 193 & 10 \\
\hline \hline
\end{tabular}

\begin{tabular}{c|c|c|c|c|c|c|c|c|c|c}
\hline \hline \multirow{2}{*}{ Year } & \multicolumn{10}{c}{ Neighborhood Concept F } \\
& \multicolumn{9}{|c|}{ Intersection (\%) } & \multicolumn{7}{c}{ Segment (\%) } \\
\cline { 2 - 12 } & K & A & B & C & O & K & A & B & C & O \\
\hline 2007 & 25 & 17 & 10 & 3 & 0 & 14 & 22 & 11 & 10 & 0 \\
\hline 2008 & 0 & 4 & 4 & 10 & 14 & 14 & 12 & 10 & 12 & 0 \\
\hline 2009 & 0 & 13 & 8 & 8 & 14 & 11 & 12 & 8 & 12 & 0 \\
\hline 2010 & 19 & 9 & 11 & 19 & 0 & 19 & 11 & 11 & 9 & 0 \\
\hline 2011 & 6 & 11 & 13 & 15 & 14 & 9 & 14 & 15 & 16 & 0 \\
\hline 2012 & 13 & 13 & 22 & 17 & 29 & 7 & 14 & 15 & 10 & 50 \\
\hline 2013 & 25 & 9 & 17 & 12 & 14 & 12 & 9 & 15 & 12 & 0 \\
\hline 2014 & 13 & 23 & 14 & 15 & 14 & 14 & 6 & 15 & 18 & 50 \\
\hline Total & 16 & 53 & 230 & 156 & 7 & 57 & 65 & 175 & 67 & 2 \\
\hline \hline
\end{tabular}

In terms of bicyclists, there were 4,702 crashes that occurred in intersections, and 864 that occurred in road segments. Most of the crashes resulted in injury B as well.

Furthermore, there has been an increase of injury A and B crashes on roads located in neighborhood concept $\mathrm{D}$ and $\mathrm{E}$ over the period studied.

The number of fatal crashes at intersections and on road segments has been similar, but for the rest of the severity levels, intersections have carried the majority of the crashes. This finding contrasted with the pedestrian findings, where most of the fatal and severe crashes were at segments. 
TABLE 13: Statewide bicyclist crashes by neighborhood concepts and year

\begin{tabular}{c|c|c|c|c|c|c|c|c|c|c}
\hline \hline & \multicolumn{10}{c}{ Neighborhood Concept C } \\
\hline \multirow{2}{*}{ Year } & \multicolumn{1}{|c}{ Intersection (\%) } & \multicolumn{7}{c}{ Segment (\%) } \\
\cline { 2 - 12 } & K & A & B & C & O & K & A & B & C & O \\
\hline 2007 & 0 & 36 & 10 & 9 & 0 & 0 & 20 & 14 & 0 & 0 \\
\hline 2008 & 0 & 18 & 7 & 18 & 0 & 0 & 0 & 7 & 0 & 0 \\
\hline 2009 & 0 & 9 & 16 & 11 & 0 & 0 & 40 & 14 & 0 & 0 \\
\hline 2010 & 0 & 9 & 12 & 14 & 50 & 0 & 20 & 14 & 0 & 0 \\
\hline 2011 & 50 & 9 & 13 & 14 & 25 & 0 & 0 & 7 & 67 & 0 \\
\hline 2012 & 50 & 9 & 17 & 5 & 0 & 0 & 0 & 14 & 0 & 50 \\
\hline 2013 & 0 & 9 & 16 & 11 & 25 & 0 & 20 & 7 & 0 & 0 \\
\hline 2014 & 0 & 0 & 10 & 18 & 0 & 0 & 0 & 21 & 33 & 50 \\
\hline Total & 2 & 11 & 94 & 44 & 4 & 0 & 5 & 14 & 3 & 2 \\
\hline \hline
\end{tabular}

\begin{tabular}{c|c|c|c|c|c|c|c|c|c|c}
\hline \hline & \multicolumn{10}{|c}{ Neighborhood Concept D } \\
\hline \multirow{2}{*}{ Year } & \multicolumn{1}{|c|}{ Intersection (\%) } & \multicolumn{7}{c}{ Segment (\%) } \\
\cline { 2 - 12 } & K & A & B & C & O & K & A & B & C & O \\
\hline 2007 & 30 & 13 & 8 & 9 & 14 & 0 & 16 & 10 & 10 & 0 \\
\hline 2008 & 20 & 20 & 11 & 11 & 10 & 0 & 9 & 15 & 14 & 8 \\
\hline 2009 & 10 & 10 & 11 & 12 & 16 & 43 & 22 & 9 & 15 & 17 \\
\hline 2010 & 10 & 8 & 15 & 12 & 12 & 0 & 6 & 11 & 18 & 17 \\
\hline 2011 & 10 & 12 & 13 & 13 & 11 & 14 & 6 & 14 & 10 & 8 \\
\hline 2012 & 0 & 16 & 14 & 16 & 7 & 14 & 9 & 17 & 13 & 17 \\
\hline 2013 & 10 & 10 & 14 & 13 & 21 & 14 & 9 & 11 & 9 & 17 \\
\hline 2014 & 0 & 12 & 15 & 14 & 14 & 14 & 22 & 15 & 11 & 17 \\
\hline Total & 10 & 145 & 1288 & 721 & 94 & 7 & 32 & 186 & 92 & 12 \\
\hline \hline
\end{tabular}

\begin{tabular}{c|c|c|c|c|c|c|c|c|c|c}
\hline \hline & \multicolumn{10}{c}{ Neighborhood Concept E } \\
\hline \multirow{2}{*}{ Year } & \multicolumn{1}{|c}{ Intersection (\%) } & \multicolumn{3}{c}{ Segment (\%) } \\
\cline { 2 - 12 } & K & A & B & C & O & K & A & B & C & O \\
\hline 2007 & 21 & 12 & 9 & 7 & 6 & 29 & 19 & 12 & 8 & 27 \\
\hline 2008 & 29 & 15 & 10 & 11 & 10 & 0 & 13 & 10 & 10 & 0 \\
\hline 2009 & 0 & 13 & 11 & 12 & 15 & 7 & 3 & 13 & 16 & 27 \\
\hline 2010 & 7 & 6 & 14 & 13 & 6 & 7 & 13 & 11 & 6 & 7 \\
\hline 2011 & 21 & 15 & 14 & 13 & 9 & 29 & 10 & 8 & 13 & 0 \\
\hline 2012 & 14 & 11 & 16 & 16 & 18 & 21 & 13 & 17 & 12 & 7 \\
\hline 2013 & 0 & 18 & 13 & 13 & 15 & 0 & 10 & 16 & 16 & 13 \\
\hline 2014 & 7 & 11 & 13 & 15 & 21 & 7 & 19 & 13 & 19 & 20 \\
\hline Total & 14 & 131 & 937 & 502 & 67 & 14 & 31 & 190 & 83 & 15 \\
\hline \hline
\end{tabular}


TABLE 13: Statewide bicyclist crashes by neighborhood concepts and year, continued

\begin{tabular}{c|c|c|c|c|c|c|c|c|c|c}
\hline \hline & \multicolumn{10}{c}{ Neighborhood Concept F } \\
\hline \multirow{2}{*}{ Year } & \multicolumn{1}{|c}{ Intersection (\%) } & \multicolumn{7}{c}{ Segment (\%) } \\
\cline { 2 - 12 } & K & A & B & C & O & K & A & B & C & O \\
\hline 2007 & 17 & 8 & 7 & 6 & 0 & 33 & 0 & 11 & 13 & 0 \\
\hline 2008 & 0 & 8 & 10 & 9 & 6 & 17 & 7 & 3 & 0 & 50 \\
\hline 2009 & 0 & 8 & 12 & 13 & 6 & 17 & 20 & 15 & 6 & 0 \\
\hline 2010 & 17 & 6 & 16 & 16 & 18 & 17 & 7 & 9 & 10 & 0 \\
\hline 2011 & 17 & 17 & 15 & 12 & 18 & 17 & 27 & 12 & 29 & 0 \\
\hline 2012 & 0 & 17 & 15 & 15 & 29 & 0 & 20 & 15 & 23 & 0 \\
\hline 2013 & 17 & 17 & 13 & 11 & 18 & 0 & 7 & 19 & 10 & 50 \\
\hline 2014 & 17 & 19 & 13 & 18 & 6 & 0 & 13 & 17 & 10 & 0 \\
\hline Total & 6 & 36 & 379 & 198 & 17 & 6 & 15 & 124 & 31 & 2 \\
\hline \hline
\end{tabular}

\subsection{Pedestrian and bicyclist characteristics}

Two variables were assessed for pedestrian and bicyclist characteristics: age and gender. For pedestrians, the findings suggested that most of the severity level $\mathrm{K}$ and A crashes involved a pedestrian older than 45 years old (for neighborhood concept E, $60 \%$ of the fatal crashes involved a pedestrian older than 55). This finding is similar to the findings from the literature review: as people get older, the likelihood of a fatal and severe crash increased due to the reduction in visibility, reaction time, and body resistance. Another vulnerable population, pedestrians younger than 12 years old, were found to made up to $9 \%$ of the fatal crashes and $13 \%$ of the severity level A crashes for the different neighborhood concepts. 
TABLE 14: Statewide pedestrian crashes (2007-2014) by severity level, neighborhood concept and vulnerable user age.

\begin{tabular}{c|c|c|c|c|c|c|c|c|c|c}
\hline \hline \multirow{2}{*}{ Age } & \multicolumn{10}{c}{ Neighborhood Concept C } \\
\cline { 2 - 12 } & \multicolumn{9}{|c}{ Intersection (\%) } & \multicolumn{7}{c|}{ Segment (\%) } \\
\cline { 2 - 12 } & K & A & B & C & O & K & A & B & C & O \\
\hline $0-12$ & 0 & 7 & 10 & 14 & 100 & 0 & 0 & 0 & 11 & 0 \\
\hline $12-18$ & 0 & 0 & 2 & 0 & 0 & 0 & 0 & 0 & 0 & 0 \\
\hline $18-24$ & 100 & 33 & 12 & 13 & 0 & 0 & 0 & 23 & 33 & 0 \\
\hline $25-34$ & 0 & 13 & 16 & 26 & 0 & 0 & 0 & 23 & 22 & 0 \\
\hline $35-44$ & 0 & 20 & 13 & 14 & 0 & 0 & 0 & 15 & 22 & 0 \\
\hline $45-54$ & 0 & 7 & 16 & 16 & 0 & 0 & 0 & 15 & 11 & 0 \\
\hline $55-64$ & 0 & 7 & 21 & 16 & 0 & 0 & 100 & 15 & 0 & 0 \\
\hline $65-74$ & 0 & 0 & 8 & 0 & 0 & 0 & 0 & 8 & 0 & 0 \\
\hline $75+$ & 0 & 13 & 1 & 1 & 0 & 0 & 0 & 0 & 0 & 0 \\
\hline Total & 2 & 15 & 86 & 70 & 1 & 0 & 1 & 13 & 9 & 0 \\
\hline \hline
\end{tabular}

\begin{tabular}{c|c|c|c|c|c|c|c|c|c|c}
\hline \hline \multirow{2}{*}{ Age } & \multicolumn{10}{c}{ Neighborhood Concept D } \\
\cline { 2 - 13 } & \multicolumn{1}{|c}{ Intersection (\%) } & \multicolumn{7}{c}{ Segment (\%) } & \\
\cline { 2 - 12 } & K & A & B & C & O & K & A & B & C & O \\
\hline $0-12$ & 3 & 13 & 10 & 12 & 31 & 4 & 14 & 17 & 18 & 40 \\
\hline $12-18$ & 1 & 13 & 10 & 9 & 6 & 0 & 0 & 0 & 0 & 0 \\
\hline $18-24$ & 10 & 9 & 16 & 15 & 31 & 4 & 13 & 12 & 8 & 20 \\
\hline $25-34$ & 9 & 13 & 17 & 16 & 25 & 18 & 9 & 14 & 17 & 0 \\
\hline $35-44$ & 13 & 12 & 10 & 15 & 6 & 4 & 15 & 14 & 16 & 20 \\
\hline $45-54$ & 16 & 16 & 13 & 15 & 0 & 16 & 13 & 12 & 11 & 0 \\
\hline $55-64$ & 15 & 11 & 12 & 10 & 0 & 26 & 16 & 14 & 15 & 20 \\
\hline $65-74$ & 16 & 8 & 7 & 5 & 0 & 18 & 13 & 9 & 8 & 0 \\
\hline $75+$ & 15 & 5 & 4 & 2 & 0 & 4 & 5 & 5 & 7 & 0 \\
\hline Total & 67 & 184 & 890 & 661 & 16 & 50 & 116 & 334 & 169 & 5 \\
\hline \hline
\end{tabular}


TABLE 14: Statewide pedestrian crashes (2007-2014) by severity level, neighborhood concept and vulnerable user age, continued

\begin{tabular}{c|c|c|c|c|c|c|c|c|c|c}
\hline \hline \multirow{2}{*}{ Age } & \multicolumn{10}{c}{ Neighborhood Concept E } \\
\cline { 2 - 12 } & \multicolumn{1}{|c}{ Intersection (\%) } & \multicolumn{4}{c}{ Segment (\%) } \\
\cline { 2 - 11 } & K & A & B & C & O & K & A & B & C & O \\
\hline $0-12$ & 9 & 11 & 13 & 10 & 31 & 3 & 9 & 17 & 14 & 20 \\
\hline $12-18$ & 4 & 18 & 19 & 13 & 6 & 2 & 10 & 13 & 17 & 10 \\
\hline $18-24$ & 13 & 14 & 14 & 16 & 13 & 13 & 17 & 18 & 14 & 10 \\
\hline $25-34$ & 11 & 11 & 13 & 17 & 19 & 11 & 13 & 12 & 16 & 20 \\
\hline $35-44$ & 11 & 12 & 9 & 14 & 6 & 14 & 11 & 10 & 10 & 0 \\
\hline $45-54$ & 11 & 14 & 11 & 13 & 19 & 22 & 20 & 13 & 13 & 40 \\
\hline $55-64$ & 14 & 7 & 11 & 9 & 0 & 19 & 13 & 11 & 10 & 0 \\
\hline $65-74$ & 16 & 6 & 6 & 5 & 0 & 4 & 3 & 4 & 6 & 0 \\
\hline $75+$ & 13 & 7 & 4 & 3 & 6 & 11 & 5 & 4 & 3 & 0 \\
\hline NA & 9 & 11 & 13 & 10 & 31 & 3 & 9 & 17 & 14 & 20 \\
\hline Total & 56 & 152 & 616 & 487 & 16 & 123 & 152 & 357 & 200 & 10 \\
\hline \hline
\end{tabular}

\begin{tabular}{c|c|c|c|c|c|c|c|c|c|c}
\hline \hline \multirow{2}{*}{ Age } & \multicolumn{10}{c}{ Neighborhood Concept F } \\
\cline { 2 - 12 } & \multicolumn{1}{|c|}{ Intersection (\%) } & \multicolumn{3}{c}{ Segment (\%) } & ( \\
\cline { 2 - 12 } & K & A & B & C & O & K & A & B & C & O \\
\hline $0-12$ & 7 & 13 & 9 & 14 & 16 & 3 & 12 & 23 & 21 & 0 \\
\hline $12-18$ & 0 & 13 & 18 & 14 & 11 & 2 & 13 & 20 & 9 & 0 \\
\hline $18-24$ & 7 & 13 & 17 & 16 & 11 & 13 & 16 & 18 & 24 & 50 \\
\hline $25-34$ & 7 & 19 & 14 & 16 & 5 & 18 & 13 & 12 & 16 & 0 \\
\hline $35-44$ & 7 & 8 & 12 & 10 & 21 & 23 & 13 & 9 & 10 & 0 \\
\hline $45-54$ & 13 & 12 & 12 & 11 & 16 & 15 & 20 & 11 & 10 & 0 \\
\hline $55-64$ & 27 & 10 & 11 & 12 & 21 & 11 & 7 & 3 & 4 & 0 \\
\hline $65-74$ & 20 & 6 & 6 & 5 & 0 & 5 & 5 & 3 & 1 & 0 \\
\hline $75+$ & 13 & 6 & 2 & 2 & 0 & 11 & 1 & 1 & 4 & 50 \\
\hline NA & 7 & 13 & 9 & 14 & 16 & 3 & 12 & 23 & 21 & 0 \\
\hline Total & 15 & 52 & 226 & 173 & 19 & 62 & 76 & 184 & 70 & 2 \\
\hline \hline
\end{tabular}

Different results were found for bicyclists. Crashes that occurred in neighborhood D were fatal or severity level A when there was a bicyclist younger than 34. For neighborhood E, most of the fatal crashes involved bicyclists over 45 , while most of the severe crashes (level A and B) involved bicyclists younger than 24. Some of the literature review 
suggested that younger people who bike accepted more risk than older bikers, which resulted in higher exposure and likelihood of being involved in a fatal or severe crash.

TABLE 15: Statewide bicyclist crashes (2007-2014) by severity level, neighborhood concept and vulnerable user age.

\begin{tabular}{c|c|c|c|c|c|c|c|c|c|c}
\hline \hline \multirow{2}{*}{ Age } & \multicolumn{10}{c}{ Neighborhood Concept C } \\
\cline { 2 - 12 } & \multicolumn{1}{|c|}{ Intersection (\%) } & \multicolumn{7}{c}{ Segment (\%) } & O \\
\cline { 2 - 12 } & K & A & B & C & O & K & A & B & C & O \\
\hline $0-12$ & 0 & 0 & 12 & 14 & 100 & 0 & 0 & 14 & 0 & 50 \\
\hline $12-18$ & 0 & 0 & 2 & 7 & 0 & 0 & 0 & 0 & 0 & 0 \\
\hline $18-24$ & 50 & 9 & 24 & 23 & 0 & 0 & 0 & 7 & 33 & 50 \\
\hline $25-34$ & 50 & 27 & 34 & 34 & 0 & 0 & 80 & 64 & 33 & 0 \\
\hline $35-44$ & 0 & 27 & 14 & 9 & 0 & 0 & 0 & 14 & 0 & 0 \\
\hline $45-54$ & 0 & 18 & 7 & 9 & 0 & 0 & 20 & 0 & 33 & 0 \\
\hline $55-64$ & 0 & 18 & 5 & 2 & 0 & 0 & 0 & 0 & 0 & 0 \\
\hline $65-74$ & 0 & 0 & 2 & 2 & 0 & 0 & 0 & 0 & 0 & 0 \\
\hline $75+$ & 0 & 0 & 0 & 0 & 0 & 0 & 0 & 0 & 0 & 0 \\
\hline Total & 2 & 11 & 95 & 44 & 4 & 0 & 5 & 14 & 3 & 2 \\
\hline \hline
\end{tabular}

\begin{tabular}{c|c|c|c|c|c|c|c|c|c|c}
\hline \hline \multirow{2}{*}{ Age } & \multicolumn{10}{c}{ Neighborhood Concept D } \\
\cline { 2 - 12 } & \multicolumn{9}{|c}{ Intersection (\%) } & \multicolumn{7}{c}{ Segment (\%) } \\
\cline { 2 - 12 } & K & A & B & C & O & K & A & B & C & O \\
\hline $0-12$ & 0 & 5 & 12 & 12 & 45 & 0 & 9 & 21 & 16 & 58 \\
\hline $12-18$ & 20 & 9 & 9 & 9 & 10 & 0 & 9 & 10 & 6 & 8 \\
\hline $18-24$ & 30 & 25 & 22 & 21 & 11 & 0 & 16 & 17 & 24 & 8 \\
\hline $25-34$ & 30 & 20 & 24 & 22 & 16 & 43 & 22 & 19 & 30 & 0 \\
\hline $35-44$ & 20 & 18 & 13 & 16 & 8 & 14 & 19 & 16 & 11 & 0 \\
\hline $45-54$ & 0 & 12 & 12 & 13 & 5 & 14 & 6 & 11 & 10 & 25 \\
\hline $55-64$ & 0 & 7 & 6 & 4 & 4 & 14 & 9 & 5 & 3 & 0 \\
\hline $65-74$ & 0 & 3 & 2 & 2 & 0 & 0 & 6 & 1 & 0 & 0 \\
\hline $75+$ & 0 & 2 & 1 & 1 & 0 & 14 & 3 & 0 & 0 & 0 \\
\hline Total & 10 & 146 & 1304 & 725 & 99 & 7 & 32 & 189 & 93 & 12 \\
\hline \hline
\end{tabular}


TABLE 15: Statewide bicyclist crashes (2007-2014) by severity level, neighborhood concept and vulnerable user age, continued

\begin{tabular}{c|c|c|c|c|c|c|c|c|c|c}
\hline \hline \multirow{2}{*}{ Age } & \multicolumn{10}{c}{ Neighborhood Concept E } \\
\cline { 2 - 11 } & \multicolumn{9}{|c}{ Intersection (\%) } & \multicolumn{7}{c}{ Segment (\%) } \\
\cline { 2 - 11 } & K & A & B & C & O & K & A & B & C & O \\
\hline $0-12$ & 0 & 9 & 13 & 14 & 33 & 0 & 16 & 21 & 11 & 27 \\
\hline $12-18$ & 13 & 10 & 15 & 11 & 12 & 7 & 19 & 14 & 10 & 20 \\
\hline $18-24$ & 7 & 20 & 20 & 18 & 20 & 0 & 13 & 12 & 14 & 20 \\
\hline $25-34$ & 0 & 14 & 17 & 18 & 13 & 7 & 6 & 20 & 23 & 13 \\
\hline $35-44$ & 20 & 11 & 12 & 13 & 6 & 29 & 16 & 10 & 13 & 7 \\
\hline $45-54$ & 27 & 18 & 11 & 16 & 7 & 14 & 19 & 11 & 14 & 13 \\
\hline $55-64$ & 7 & 11 & 7 & 7 & 7 & 14 & 6 & 7 & 11 & 0 \\
\hline $65-74$ & 20 & 4 & 3 & 3 & 1 & 21 & 6 & 4 & 1 & 0 \\
\hline $75+$ & 7 & 4 & 1 & 0 & 0 & 7 & 0 & 2 & 2 & 0 \\
\hline NA & 0 & 9 & 13 & 14 & 33 & 0 & 16 & 21 & 11 & 27 \\
\hline Total & 15 & 131 & 950 & 505 & 69 & 14 & 32 & 191 & 83 & 15 \\
\hline \hline
\end{tabular}

\begin{tabular}{c|c|c|c|c|c|c|c|c|c|c}
\hline \hline \multirow{2}{*}{ Age } & \multicolumn{10}{c}{ Neighborhood Concept F } \\
\cline { 2 - 12 } & \multicolumn{1}{|c}{ Intersection (\%) } & \multicolumn{7}{c}{ Segment (\%) } \\
\cline { 2 - 12 } & K & A & B & C & O & K & A & B & C & O \\
\hline $0-12$ & 0 & 11 & 15 & 17 & 53 & 17 & 7 & 24 & 16 & 0 \\
\hline $12-18$ & 20 & 17 & 13 & 10 & 11 & 0 & 13 & 15 & 6 & 0 \\
\hline $18-24$ & 0 & 17 & 17 & 15 & 11 & 0 & 20 & 16 & 19 & 50 \\
\hline $25-34$ & 0 & 11 & 16 & 15 & 0 & 17 & 7 & 12 & 10 & 0 \\
\hline $35-44$ & 0 & 14 & 14 & 19 & 11 & 17 & 0 & 5 & 19 & 50 \\
\hline $45-54$ & 20 & 14 & 15 & 13 & 16 & 50 & 33 & 12 & 16 & 0 \\
\hline $55-64$ & 40 & 14 & 6 & 10 & 0 & 0 & 7 & 10 & 10 & 0 \\
\hline $65-74$ & 20 & 0 & 3 & 3 & 0 & 0 & 13 & 5 & 3 & 0 \\
\hline $75+$ & 0 & 3 & 1 & 0 & 0 & 0 & 0 & 2 & 0 & 0 \\
\hline NA & 0 & 11 & 15 & 17 & 53 & 17 & 7 & 24 & 16 & 0 \\
\hline Total & 5 & 36 & 385 & 199 & 19 & 6 & 15 & 129 & 31 & 2 \\
\hline \hline
\end{tabular}

In terms of gender, it was found that men were involved in more fatal and severe crashes than women were. This result was consistent for intersections and segments, and the different neighborhood concepts. Based on the literature review, there was no consensus on this topic, since studies found contradictory results or non-significant findings. 
Although there was a trend in terms of gender in this descriptive analysis, more exploration is needed to determine the role of gender.

TABLE 16: Statewide pedestrian crashes (2007-2014) by severity level, neighborhood concept and vulnerable user gender.

\begin{tabular}{c|c|c|c|c|c|c|c|c|c|c}
\hline \hline & \multicolumn{10}{c}{ Neighborhood Concept C } \\
\cline { 2 - 13 } Gender & \multicolumn{1}{|c|}{ Intersection (\%) } & \multicolumn{7}{c}{ Segment (\%) } \\
\cline { 2 - 13 } & $\mathbf{K}$ & $\mathbf{A}$ & $\mathbf{B}$ & $\mathbf{C}$ & $\mathbf{O}$ & $\mathbf{K}$ & $\mathbf{A}$ & $\mathbf{B}$ & $\mathbf{C}$ & $\mathbf{O}$ \\
\hline Male & 0 & 53 & 58 & 44 & 0 & 0 & 62 & 78 & 0 & 0 \\
\hline Female & 100 & 47 & 41 & 55 & 0 & 100 & 38 & 22 & 0 & 0 \\
\hline Unknown & 0 & 0 & 1 & 1 & 100 & 0 & 0 & 0 & 0 & 0 \\
\hline Total & 2 & 15 & 83 & 73 & 1 & 1 & 13 & 9 & 0 & 0 \\
\hline \hline
\end{tabular}

\begin{tabular}{|c|c|c|c|c|c|c|c|c|c|c|}
\hline \multirow[b]{3}{*}{ Gender } & \multicolumn{10}{|c|}{ Neighborhood Concept D } \\
\hline & \multicolumn{5}{|c|}{ Intersection (\%) } & \multicolumn{5}{|c|}{ Segment $(\%)$} \\
\hline & $\mathbf{K}$ & $\mathbf{A}$ & $\mathbf{B}$ & C & $\mathbf{O}$ & $\mathbf{K}$ & $\mathbf{A}$ & B & $\mathbf{C}$ & $\mathbf{O}$ \\
\hline Male & 60 & 58 & 52 & 51 & 63 & 62 & 66 & 58 & 63 & 0 \\
\hline Female & 40 & 42 & 48 & 48 & 31 & 38 & 34 & 41 & 36 & 80 \\
\hline Unknown & 0 & 0 & 0 & 1 & 6 & 0 & 0 & 1 & 1 & 20 \\
\hline Total & 67 & 184 & 890 & 661 & 16 & 50 & 116 & 334 & 169 & 5 \\
\hline
\end{tabular}

\begin{tabular}{|c|c|c|c|c|c|c|c|c|c|c|}
\hline \multirow[b]{3}{*}{ Gender } & \multicolumn{10}{|c|}{ Neighborhood Concept E } \\
\hline & \multicolumn{5}{|c|}{ Intersection (\%) } & \multicolumn{5}{|c|}{ Segment $(\%)$} \\
\hline & $\mathbf{K}$ & $\mathbf{A}$ & $\mathbf{B}$ & $\mathbf{C}$ & $\mathbf{O}$ & $\mathbf{K}$ & $\mathbf{A}$ & B & $\mathbf{C}$ & $\mathbf{O}$ \\
\hline Male & 71 & 59 & 52 & 49 & 69 & 72 & 73 & 61 & 65 & 30 \\
\hline Female & 29 & 41 & 48 & 51 & 25 & 28 & 27 & 38 & 34 & 70 \\
\hline Unknown & 0 & 0 & 0 & 1 & 6 & 0 & 0 & 1 & 2 & 0 \\
\hline Total & 56 & 152 & 616 & 487 & 16 & 123 & 152 & 357 & 200 & 10 \\
\hline
\end{tabular}

\begin{tabular}{|c|c|c|c|c|c|c|c|c|c|c|}
\hline \multirow{3}{*}{ Gender } & \multicolumn{10}{|c|}{ Neighborhood Concept E } \\
\hline & \multicolumn{5}{|c|}{ Intersection (\%) } & \multicolumn{5}{|c|}{ Segment $(\%)$} \\
\hline & $\mathbf{K}$ & $\mathbf{A}$ & B & $\mathbf{C}$ & $\mathbf{O}$ & $\mathbf{K}$ & $\mathbf{A}$ & B & $\mathbf{C}$ & $\mathbf{O}$ \\
\hline Male & 67 & 62 & 56 & 48 & 58 & 74 & 63 & 67 & 61 & 50 \\
\hline Female & 33 & 38 & 43 & 50 & 42 & 26 & 37 & 32 & 36 & 50 \\
\hline Unknown & 0 & 0 & 0 & 2 & 0 & 0 & 0 & 1 & 3 & 0 \\
\hline Total & 15 & 52 & 226 & 173 & 19 & 62 & 76 & 184 & 70 & 2 \\
\hline
\end{tabular}


Similar results were found for bicyclists. For all the severity levels and neighborhood concepts, there were more crashes for men than women. Furthermore, similar proportions of crashes between men and women were found in neighborhood concepts $\mathrm{C}$ and $\mathrm{F}$; however, there are not too many observations in these land use typologies.

TABLE 17: Statewide bicyclist crashes (2007-2014) by severity level, neighborhood concept and vulnerable user gender.

\begin{tabular}{c|c|c|c|c|c|c|c|c|c|c}
\hline \hline & \multicolumn{10}{c}{ Neighborhood Concept C } \\
\cline { 2 - 12 } Gender & \multicolumn{1}{|c}{ Intersection (\%) } & \multicolumn{7}{c}{ Segment (\%) } \\
\cline { 2 - 12 } & $\mathbf{K}$ & $\mathbf{A}$ & $\mathbf{B}$ & $\mathbf{C}$ & $\mathbf{O}$ & $\mathbf{K}$ & $\mathbf{A}$ & $\mathbf{B}$ & $\mathbf{C}$ & $\mathbf{O}$ \\
\hline Male & 50 & 73 & 68 & 68 & 50 & 0 & 80 & 79 & 67 & 50 \\
\hline Female & 50 & 27 & 31 & 32 & 50 & 0 & 20 & 21 & 33 & 50 \\
\hline Unknown & 0 & 0 & 1 & 0 & 0 & 0 & 0 & 0 & 0 & 0 \\
\hline Total & 2 & 11 & 95 & 44 & 4 & 0 & 5 & 14 & 3 & 2 \\
\hline \hline
\end{tabular}

\begin{tabular}{c|c|c|c|c|c|c|c|c|c|c}
\hline \hline & \multicolumn{10}{c}{ Neighborhood Concept D } \\
\cline { 2 - 12 } Gender & \multicolumn{1}{|c}{ Intersection (\%) } & \multicolumn{7}{c}{ Segment (\%) } \\
\cline { 2 - 13 } & K & A & B & C & O & K & A & B & C & O \\
\hline Male & 80 & 73 & 70 & 68 & 76 & 86 & 66 & 71 & 71 & 75 \\
\hline Female & 20 & 27 & 29 & 31 & 16 & 14 & 34 & 27 & 29 & 8 \\
\hline Unknown & 0 & 0 & 0 & 1 & 8 & 0 & 0 & 2 & 0 & 17 \\
\hline Total & 10 & 146 & 1304 & 725 & 99 & 7 & 32 & 189 & 93 & 12 \\
\hline \hline
\end{tabular}

\begin{tabular}{|c|c|c|c|c|c|c|c|c|c|c|}
\hline \multirow[b]{3}{*}{ Gender } & \multicolumn{10}{|c|}{ Neighborhood Concept E } \\
\hline & \multicolumn{5}{|c|}{ Intersection (\%) } & \multicolumn{5}{|c|}{ Segment $(\%)$} \\
\hline & $\mathbf{K}$ & $\mathbf{A}$ & B & $\mathbf{C}$ & $\mathbf{O}$ & $\mathbf{K}$ & $\mathbf{A}$ & $\mathbf{B}$ & $\mathbf{C}$ & $\mathbf{O}$ \\
\hline Male & 80 & 79 & 75 & 74 & 81 & 86 & 78 & 85 & 84 & 93 \\
\hline Female & 20 & 21 & 25 & 26 & 14 & 14 & 19 & 14 & 16 & 7 \\
\hline Unknown & 0 & 0 & 0 & 0 & 4 & 0 & 3 & 1 & 0 & 0 \\
\hline Total & 15 & 131 & 950 & 505 & 69 & 14 & 32 & 191 & 83 & 15 \\
\hline
\end{tabular}


TABLE 17: Statewide bicyclist crashes (2007-2014) by severity level, neighborhood concept and vulnerable user gender, continued

\begin{tabular}{c|c|c|c|c|c|c|c|c|c|c}
\hline \hline \multirow{3}{*}{ Gender } & \multicolumn{10}{c}{ Neighborhood Concept F } \\
\cline { 2 - 12 } & \multicolumn{1}{|c}{ Intersection (\%) } & \multicolumn{7}{c}{ Segment (\%) } \\
\cline { 2 - 11 } & K & A & B & C & O & K & A & B & C & O \\
\hline Male & 60 & 78 & 77 & 76 & 58 & 83 & 87 & 77 & 77 & 100 \\
\hline Female & 40 & 22 & 22 & 23 & 16 & 17 & 13 & 23 & 13 & 0 \\
\hline Unknown & 0 & 0 & 0 & 1 & 26 & 0 & 0 & 0 & 10 & 0 \\
\hline Total & 5 & 36 & 385 & 199 & 19 & 6 & 15 & 129 & 31 & 2 \\
\hline \hline
\end{tabular}

\subsection{Road characteristics}

For road characteristics, we explored the key variables found in the literature review: road classification, posted speed limit, number of lanes, road surface, and type of intersection. Width was also found to be statistically significant in most of the studies; however, information was not available in the crash database. Width is assessed in the exposure analysis.

In terms of road classification, the descriptive analysis suggested that most of the pedestrian crashes occurred on arterials (principal and minor). This result was consistent for all neighborhood concepts regardless the severity level. Furthermore, most of the fatal crashes occurred on principal arterials, and were mainly located in neighborhood concepts D and E. Finally, most of the fatal and severe crashes occurred on principal and minor arterials. These crash observations were higher than for intersections connecting roads with the same classifications. For the other severity levels $(\mathrm{B}, \mathrm{C}, \mathrm{O})$, intersections had more crashes. 
TABLE 18: Statewide pedestrian crashes (2007-2014) by severity level, neighborhood concept and road classification.

\begin{tabular}{c|c|c|c|c|c|c|c|c|c|c|}
\hline \hline \multirow{2}{*}{$\begin{array}{c}\text { Road } \\
\text { Classification }\end{array}$} & \multicolumn{10}{c|}{ Neighborhood Concept C } \\
\cline { 2 - 11 } & \multicolumn{9}{|c|}{ Intersection (\%) } & \multicolumn{7}{c|}{ Segment (\%) } \\
\cline { 2 - 11 } & K & A & B & C & O & K & A & B & C & O \\
\hline $\begin{array}{c}\text { Interstate/ } \\
\text { Freeway }\end{array}$ & 0 & 0 & 1 & 0 & 0 & 0 & 0 & 0 & 0 & 0 \\
\hline $\begin{array}{c}\text { Principal } \\
\text { Arterial }\end{array}$ & 0 & 43 & 17 & 13 & 0 & 0 & 100 & 69 & 22 & 0 \\
\hline $\begin{array}{c}\text { Minor } \\
\text { Arterial }\end{array}$ & 100 & 43 & 54 & 51 & 0 & 0 & 0 & 8 & 33 & 0 \\
\hline $\begin{array}{c}\text { Rural } \\
\text { collector }\end{array}$ & 0 & 0 & 0 & 0 & 0 & 0 & 0 & 0 & 0 & 0 \\
\hline $\begin{array}{c}\text { Urban } \\
\text { collector }\end{array}$ & 0 & 14 & 23 & 35 & 100 & 0 & 0 & 23 & 33 & 0 \\
\hline Local & 0 & 0 & 4 & 0 & 0 & 0 & 0 & 0 & 11 & 0 \\
\hline Total & 1 & 14 & 81 & 68 & 1 & 0 & 1 & 13 & 9 & 0 \\
\hline \hline
\end{tabular}

\begin{tabular}{|c|c|c|c|c|c|c|c|c|c|c|}
\hline \multirow{3}{*}{$\begin{array}{c}\text { Road } \\
\text { Classification }\end{array}$} & \multicolumn{10}{|c|}{ Neighborhood Concept D } \\
\hline & \multicolumn{5}{|c|}{ Intersection (\%) } & \multicolumn{5}{|c|}{ Segment (\%) } \\
\hline & $\mathbf{K}$ & $\mathbf{A}$ & B & $\mathrm{C}$ & $\mathbf{O}$ & $\mathbf{K}$ & $\mathbf{A}$ & B & $\mathbf{C}$ & $\mathbf{O}$ \\
\hline $\begin{array}{l}\text { Interstate/ } \\
\text { Freeway }\end{array}$ & 4 & 1 & 1 & 1 & 6 & 11 & 2 & 2 & 1 & 0 \\
\hline $\begin{array}{c}\text { Principal } \\
\text { Arterial }\end{array}$ & 59 & 49 & 38 & 42 & 38 & 57 & 49 & 33 & 33 & 40 \\
\hline $\begin{array}{c}\text { Minor } \\
\text { Arterial }\end{array}$ & 28 & 28 & 33 & 31 & 19 & 15 & 26 & 28 & 21 & 0 \\
\hline $\begin{array}{c}\text { Rural } \\
\text { collector }\end{array}$ & 0 & 0 & 0 & 0 & 0 & 0 & 0 & 0 & 0 & 0 \\
\hline $\begin{array}{l}\text { Urban } \\
\text { collector }\end{array}$ & 9 & 17 & 20 & 16 & 25 & 11 & 19 & 20 & 26 & 20 \\
\hline Local & 0 & 6 & 9 & 10 & 13 & 7 & 5 & 17 & 19 & 40 \\
\hline Total & 54 & 173 & 852 & 638 & 16 & 46 & 111 & 298 & 160 & 5 \\
\hline
\end{tabular}


TABLE 18: Statewide pedestrian crashes (2007-2014) by severity level, neighborhood concept and road classification, continued

\begin{tabular}{c|c|c|c|c|c|c|c|c|c|c|}
\hline \hline \multirow{2}{*}{$\begin{array}{c}\text { Road } \\
\text { Classifi- } \\
\text { cation }\end{array}$} & \multicolumn{9}{c|}{ Intersection (\%) } & \multicolumn{7}{c|}{ Seighborhood Concept E } \\
\cline { 2 - 11 } & $\mathbf{~ K ~}$ & A & B & C & O & K & A & B & C & O \\
\hline $\begin{array}{c}\text { Interst/ } \\
\text { Freeway }\end{array}$ & 4 & 1 & 1 & 1 & 6 & 15 & 4 & 3 & 4 & 10 \\
\hline $\begin{array}{c}\text { Principal } \\
\text { Arterial }\end{array}$ & 54 & 49 & 40 & 44 & 38 & 56 & 44 & 37 & 33 & 40 \\
\hline $\begin{array}{c}\text { Minor } \\
\text { Arterial }\end{array}$ & 21 & 32 & 27 & 25 & 31 & 12 & 24 & 22 & 23 & 10 \\
\hline $\begin{array}{c}\text { Rural } \\
\text { collector }\end{array}$ & 4 & 1 & 3 & 2 & 0 & 4 & 2 & 5 & 5 & 0 \\
\hline $\begin{array}{c}\text { Urban } \\
\text { collector }\end{array}$ & 10 & 11 & 21 & 17 & 13 & 7 & 16 & 17 & 17 & 20 \\
\hline Local & 6 & 8 & 9 & 10 & 13 & 6 & 10 & 17 & 19 & 20 \\
\hline Total & 48 & 146 & 588 & 471 & 16 & 117 & 148 & 345 & 193 & 10 \\
\hline \hline
\end{tabular}

\begin{tabular}{|c|c|c|c|c|c|c|c|c|c|c|}
\hline \multirow{3}{*}{$\begin{array}{c}\text { Road } \\
\text { Classification }\end{array}$} & \multicolumn{10}{|c|}{ Neighborhood Concept F } \\
\hline & \multicolumn{5}{|c|}{ Intersection (\%) } & \multicolumn{5}{|c|}{ Segment $(\%)$} \\
\hline & $\mathbf{K}$ & $\mathbf{A}$ & B & $\mathrm{C}$ & $\mathbf{O}$ & $\mathbf{K}$ & $\mathbf{A}$ & B & $\mathbf{C}$ & $\mathbf{O}$ \\
\hline $\begin{array}{l}\text { Interstate/ } \\
\text { Freeway }\end{array}$ & 0 & 0 & 0 & 0 & 0 & 12 & 5 & 7 & 9 & 0 \\
\hline $\begin{array}{c}\text { Principal } \\
\text { Arterial }\end{array}$ & 44 & 28 & 37 & 38 & 29 & 26 & 34 & 23 & 27 & 0 \\
\hline $\begin{array}{c}\text { Minor } \\
\text { Arterial }\end{array}$ & 31 & 38 & 33 & 35 & 29 & 28 & 20 & 27 & 24 & 0 \\
\hline $\begin{array}{c}\text { Rural } \\
\text { collector }\end{array}$ & 19 & 2 & 3 & 2 & 0 & 16 & 8 & 9 & 4 & 0 \\
\hline $\begin{array}{c}\text { Urban } \\
\text { collector }\end{array}$ & 6 & 21 & 17 & 17 & 29 & 12 & 18 & 15 & 15 & 0 \\
\hline Local & 0 & 11 & 10 & 8 & 14 & 5 & 15 & 18 & 21 & 100 \\
\hline Total & 16 & 53 & 230 & 156 & 7 & 57 & 65 & 175 & 67 & 2 \\
\hline
\end{tabular}

In terms of bicyclist crashes, the results differed by the different neighborhood concepts.

In neighborhood concept $\mathrm{C}$, fatal crashes and crashes with injury level A occurred mainly on intersections with a collector leg. For segments, these crashes were mainly located on minor arterials. For concept D, most of the crashes occurred on arterials, with a majority of fatal crashes occurring on principal arterials and the majority of non-fatal crashes on 
minor arterials (for both intersections and segments). On the other hand, most of the crashes in neighborhood concept E occurred on principal arterials, regardless of the severity level. Furthermore, for concept F, the findings suggested that most of the crashes occurred on rural collectors $(80 \%)$.

As expected, most of the crashes occurred on arterials; however, minor arterials were revealed to be a significant problem since a substantial number of fatal and severe crashes occurred there.

TABLE 19: Statewide bicyclist crashes (2007-2014) by severity level, neighborhood concept and road classification.

\begin{tabular}{|c|c|c|c|c|c|c|c|c|c|c|}
\hline \multirow{3}{*}{$\begin{array}{c}\text { Road } \\
\text { Classification }\end{array}$} & \multicolumn{10}{|c|}{ Neighborhood Concept C } \\
\hline & \multicolumn{5}{|c|}{ Intersection (\%) } & \multicolumn{5}{|c|}{ Segment $(\%)$} \\
\hline & $\mathbf{K}$ & $\mathbf{A}$ & B & $\mathrm{C}$ & $\mathbf{O}$ & $\mathbf{K}$ & $\mathbf{A}$ & B & $\mathbf{C}$ & $\mathbf{O}$ \\
\hline $\begin{array}{l}\text { Interstate/ } \\
\text { Freeway }\end{array}$ & 0 & 0 & 0 & 0 & 0 & 0 & 0 & 0 & 0 & 0 \\
\hline $\begin{array}{c}\text { Principal } \\
\text { Arterial }\end{array}$ & 0 & 18 & 21 & 25 & 0 & 0 & 0 & 29 & 0 & 50 \\
\hline $\begin{array}{c}\text { Minor } \\
\text { Arterial }\end{array}$ & 50 & 36 & 40 & 41 & 75 & 0 & 100 & 43 & 33 & 0 \\
\hline $\begin{array}{c}\text { Rural } \\
\text { collector }\end{array}$ & 0 & 0 & 0 & 0 & 0 & 0 & 0 & 0 & 0 & 0 \\
\hline $\begin{array}{l}\text { Urban } \\
\text { collector }\end{array}$ & 50 & 45 & 35 & 32 & 25 & 0 & 0 & 29 & 33 & 50 \\
\hline Local & 0 & 0 & 3 & 2 & 0 & 0 & 0 & 0 & 33 & 0 \\
\hline Total & 2 & 11 & 94 & 44 & 4 & 0 & 5 & 14 & 3 & 2 \\
\hline
\end{tabular}


TABLE 19: Statewide bicyclist crashes (2007-2014) by severity level, neighborhood concept and road classification, continued

\begin{tabular}{|c|c|c|c|c|c|c|c|c|c|c|}
\hline \multirow{3}{*}{$\begin{array}{c}\text { Road } \\
\text { Classification }\end{array}$} & \multicolumn{10}{|c|}{ Neighborhood Concept D } \\
\hline & \multicolumn{5}{|c|}{ Intersection (\%) } & \multicolumn{5}{|c|}{ Segment (\%) } \\
\hline & $\mathbf{K}$ & $\mathbf{A}$ & B & $\mathbf{C}$ & $\mathbf{O}$ & $\mathbf{K}$ & $\mathbf{A}$ & B & $\mathbf{C}$ & $\mathbf{O}$ \\
\hline $\begin{array}{l}\text { Interstate/ } \\
\text { Freeway }\end{array}$ & 0 & 2 & 1 & 1 & 1 & 14 & 3 & 0 & 0 & 0 \\
\hline $\begin{array}{c}\text { Principal } \\
\text { Arterial }\end{array}$ & 33 & 32 & 30 & 32 & 29 & 57 & 16 & 26 & 25 & 33 \\
\hline $\begin{array}{l}\text { Minor } \\
\text { Arterial }\end{array}$ & 22 & 34 & 31 & 32 & 32 & 0 & 38 & 33 & 33 & 33 \\
\hline $\begin{array}{c}\text { Rural } \\
\text { collector }\end{array}$ & 0 & 0 & 0 & 0 & 0 & 0 & 0 & 0 & 0 & 0 \\
\hline $\begin{array}{c}\text { Urban } \\
\text { collector }\end{array}$ & 33 & 20 & 22 & 20 & 20 & 29 & 31 & 24 & 23 & 17 \\
\hline Local & 11 & 11 & 16 & 15 & 18 & 0 & 13 & 17 & 20 & 17 \\
\hline Total & 9 & 145 & 1288 & 721 & 98 & 7 & 32 & 186 & 92 & 12 \\
\hline
\end{tabular}

\begin{tabular}{c|c|c|c|c|c|c|c|c|c|c|}
\hline \hline \multirow{2}{*}{$\begin{array}{c}\text { Road } \\
\text { Classification }\end{array}$} & \multicolumn{8}{|c|}{ Neighborhood Concept E } \\
\cline { 2 - 12 } & \multicolumn{7}{|c|}{ Intersection (\%) } & \multicolumn{7}{c|}{ Segment (\%) } \\
\cline { 2 - 12 } & K & A & B & C & O & K & A & B & C & O \\
\hline $\begin{array}{c}\text { Interstate/ } \\
\text { Freeway }\end{array}$ & 0 & 1 & 2 & 1 & 4 & 7 & 0 & 1 & 0 & 0 \\
\hline $\begin{array}{c}\text { Principal } \\
\text { Arterial }\end{array}$ & 64 & 31 & 34 & 38 & 42 & 50 & 35 & 33 & 35 & 13 \\
\hline $\begin{array}{c}\text { Minor } \\
\text { Arterial }\end{array}$ & 21 & 34 & 31 & 31 & 24 & 36 & 35 & 23 & 20 & 27 \\
\hline $\begin{array}{c}\text { Rural } \\
\text { collector }\end{array}$ & 7 & 3 & 2 & 1 & 0 & 7 & 13 & 8 & 7 & 7 \\
\hline $\begin{array}{c}\text { Urban } \\
\text { collector }\end{array}$ & 7 & 18 & 19 & 16 & 16 & 0 & 10 & 16 & 17 & 27 \\
\hline Local & 0 & 13 & 12 & 12 & 13 & 0 & 6 & 19 & 20 & 27 \\
\hline Total & 14 & 131 & 937 & 502 & 67 & 14 & 31 & 190 & 83 & 15 \\
\hline \hline
\end{tabular}


TABLE 19: Statewide bicyclist crashes (2007-2014) by severity level, neighborhood concept and road classification, continued

\begin{tabular}{c|c|c|c|c|c|c|c|c|c|c|}
\hline \hline \multirow{2}{*}{$\begin{array}{c}\text { Road } \\
\text { Classification }\end{array}$} & \multicolumn{8}{|c|}{ Neighborhood Concept F } \\
\cline { 2 - 12 } & \multicolumn{9}{|c|}{ Intersection (\%) } & \multicolumn{7}{c|}{ Segment (\%) } \\
\cline { 2 - 12 } & K & A & B & C & O & K & A & B & C & O \\
\hline $\begin{array}{c}\text { Interstate/ } \\
\text { Freeway }\end{array}$ & 0 & 0 & 1 & 2 & 0 & 0 & 0 & 0 & 0 & 0 \\
\hline $\begin{array}{c}\text { Principal } \\
\text { Arterial }\end{array}$ & 20 & 31 & 25 & 29 & 29 & 17 & 7 & 19 & 29 & 50 \\
\hline $\begin{array}{c}\text { Minor } \\
\text { Arterial }\end{array}$ & 0 & 31 & 37 & 33 & 35 & 67 & 47 & 24 & 29 & 50 \\
\hline $\begin{array}{c}\text { Rural } \\
\text { collector }\end{array}$ & 80 & 6 & 4 & 2 & 0 & 17 & 7 & 15 & 6 & 0 \\
\hline $\begin{array}{c}\text { Urban } \\
\text { collector }\end{array}$ & 0 & 25 & 21 & 21 & 29 & 0 & 20 & 23 & 23 & 0 \\
\hline Local & 0 & 8 & 12 & 13 & 6 & 0 & 20 & 19 & 13 & 0 \\
\hline Total & 5 & 36 & 379 & 198 & 17 & 6 & 15 & 124 & 31 & 2 \\
\hline \hline
\end{tabular}

In terms of posted speed limit, the database contained a substantial number of crashes without this information. Using the data available, we found that fatal pedestrian crashes tended to occur when the major road has a posted speed limit between 35 to $50 \mathrm{mph}$. For non-fatal crashes, the majority of them occurred on roads with posted speed limits between 20 to $35 \mathrm{mph}$. This result was consistent across the neighborhood concepts. For segments, the distribution was different. Most of the crashes occurred on roads with a posted speed limit above $35 \mathrm{mph}$, regardless of severity level. For neighborhood concept $\mathrm{E}$ and $\mathrm{C}$, non-severe crashes occurred on roads with a lower posted speed limit. 
TABLE 20: Statewide pedestrian crashes (2007-2014) by severity level, neighborhood concept and posted speed limit.

\begin{tabular}{|c|c|c|c|c|c|c|c|c|c|c|}
\hline \multirow{3}{*}{$\begin{array}{l}\text { Posted } \\
\text { Speed } \\
\text { Limit } \\
\text { PSL } \\
(\mathrm{mph})\end{array}$} & \multicolumn{10}{|c|}{ Neighborhood Concept C } \\
\hline & \multicolumn{5}{|c|}{ Intersection $(\%)$} & \multicolumn{5}{|c|}{ Segment (\%) } \\
\hline & $\mathbf{K}$ & $\mathbf{A}$ & B & $\mathrm{C}$ & $\mathbf{O}$ & $\mathbf{K}$ & $\mathbf{A}$ & B & $\mathbf{C}$ & $\mathbf{O}$ \\
\hline NA & 0 & 50 & 63 & 69 & 100 & 0 & 0 & 69 & 78 & 0 \\
\hline$<20$ & 0 & 7 & 1 & 0 & 0 & 0 & 0 & 0 & 0 & 0 \\
\hline $20-35$ & 100 & 29 & 31 & 10 & 0 & 0 & 100 & 15 & 22 & 0 \\
\hline $35-50$ & 0 & 7 & 0 & 9 & 0 & 0 & 0 & 8 & 0 & 0 \\
\hline $50-65$ & 0 & 7 & 5 & 12 & 0 & 0 & 0 & 8 & 0 & 0 \\
\hline$>65$ & 0 & 0 & 0 & 0 & 0 & 0 & 0 & 0 & 0 & 0 \\
\hline Total & 1 & 14 & 81 & 68 & 1 & 0 & 1 & 13 & 9 & 0 \\
\hline
\end{tabular}

\begin{tabular}{c|c|c|c|c|c|c|c|c|c|c|}
\hline \hline \multirow{2}{*}{$\begin{array}{c}\text { PSL } \\
(\mathbf{m p h})\end{array}$} & \multicolumn{10}{c|}{ Neighborhood Concept D } \\
\cline { 2 - 12 } & \multicolumn{9}{|c|}{ Intersection (\%) } & \multicolumn{7}{c|}{ Segment (\%) } \\
\cline { 2 - 11 } & K & A & B & C & O & K & A & B & C & O \\
\hline NA & 17 & 41 & 44 & 56 & 69 & 9 & 40 & 49 & 54 & 100 \\
\hline$<20$ & 2 & 3 & 4 & 2 & 6 & 0 & 1 & 1 & 1 & 0 \\
\hline $20-35$ & 30 & 29 & 30 & 23 & 19 & 15 & 19 & 25 & 18 & 0 \\
\hline $35-50$ & 50 & 23 & 20 & 16 & 6 & 65 & 37 & 23 & 23 & 0 \\
\hline $50-65$ & 2 & 3 & 2 & 4 & 0 & 9 & 4 & 1 & 4 & 0 \\
\hline$>65$ & 0 & 0 & 0 & 0 & 0 & 2 & 0 & 0 & 0 & 0 \\
\hline Total & 54 & 173 & 852 & 638 & 16 & 46 & 111 & 298 & 160 & 5 \\
\hline \hline
\end{tabular}

\begin{tabular}{c|c|c|c|c|c|c|c|c|c|c|}
\hline \hline \multirow{2}{*}{$\begin{array}{c}\text { PSL } \\
(\mathbf{m p h})\end{array}$} & \multicolumn{10}{c|}{ Neighborhood Concept E } \\
\cline { 2 - 12 } & \multicolumn{9}{|c|}{ Intersection (\%) } & \multicolumn{7}{c|}{ Segment (\%) } \\
\cline { 2 - 12 } & K & A & B & C & O & K & A & B & C & O \\
\hline NA & 19 & 37 & 39 & 41 & 50 & 15 & 29 & 36 & 39 & 50 \\
\hline$<20$ & 2 & 1 & 1 & 1 & 0 & 1 & 1 & 1 & 2 & 0 \\
\hline $20-35$ & 23 & 25 & 31 & 29 & 19 & 9 & 18 & 23 & 27 & 20 \\
\hline $35-50$ & 50 & 34 & 28 & 26 & 31 & 46 & 39 & 31 & 22 & 20 \\
\hline $50-65$ & 6 & 3 & 1 & 3 & 0 & 23 & 13 & 8 & 8 & 10 \\
\hline$>65$ & 0 & 0 & 0 & 0 & 0 & 6 & 1 & 2 & 2 & 0 \\
\hline Total & 48 & 146 & 588 & 471 & 16 & 117 & 148 & 345 & 193 & 10 \\
\hline \hline
\end{tabular}


TABLE 20: Statewide pedestrian crashes (2007-2014) by severity level, neighborhood concept and posted speed limit, continued

\begin{tabular}{c|c|c|c|c|c|c|c|c|c|c|}
\hline \hline \multirow{2}{*}{$\begin{array}{c}\text { PSL } \\
(\mathbf{m p h})\end{array}$} & \multicolumn{10}{c|}{ Neighborhood Concept F } \\
\cline { 2 - 12 } & \multicolumn{9}{|c|}{ Intersection (\%) } & \multicolumn{7}{c|}{ Segment (\%) } \\
\cline { 2 - 12 } & K & A & B & C & O & K & A & B & C & O \\
\hline NA & 13 & 30 & 39 & 42 & 57 & 19 & 23 & 39 & 39 & 0 \\
\hline$<20$ & 6 & 4 & 2 & 3 & 0 & 2 & 0 & 3 & 1 & 100 \\
\hline $20-35$ & 19 & 32 & 28 & 27 & 29 & 4 & 20 & 18 & 16 & 0 \\
\hline $35-50$ & 50 & 28 & 28 & 24 & 0 & 33 & 28 & 21 & 27 & 0 \\
\hline $50-65$ & 13 & 6 & 3 & 5 & 14 & 33 & 28 & 16 & 12 & 0 \\
\hline$>65$ & 0 & 0 & 0 & 0 & 0 & 9 & 2 & 4 & 4 & 0 \\
\hline Total & 16 & 53 & 230 & 156 & 7 & 57 & 65 & 175 & 67 & 2 \\
\hline \hline
\end{tabular}

In terms of bicyclists, there were more fatal crashes at segments and intersections with posted speed limits between 35 to $50 \mathrm{mph}$. However, for concept E, this value was higher on roads with higher posted speed limits (above $50 \mathrm{mph}$ ). As in the pedestrian crash descriptive analysis, bicyclist crashes tended to increase as the posted speed limit increased until it reached $50 \mathrm{mph}$. After this threshold, the number of crashes became insignificant since bicycle volumes were low in these facilities (low exposure).

TABLE 21: Statewide bicyclist crashes (2007-2014) by severity level, neighborhood concept and posted speed limit.

\begin{tabular}{c|c|c|c|c|c|c|c|c|c|c|}
\hline \hline \multirow{2}{*}{$\begin{array}{c}\text { PSL } \\
(\mathbf{m p h})\end{array}$} & \multicolumn{10}{c|}{ Neighborhood Concept C } \\
\cline { 2 - 12 } & \multicolumn{9}{|c|}{ Intersection (\%) } & \multicolumn{7}{c|}{ Segment (\%) } \\
\cline { 2 - 12 } & K & A & B & C & O & K & A & B & C & O \\
\hline NA & 0 & 91 & 69 & 82 & 75 & 0 & 100 & 71 & 100 & 0 \\
\hline$<20$ & 0 & 0 & 6 & 2 & 0 & 0 & 0 & 0 & 0 & 50 \\
\hline $20-35$ & 50 & 0 & 15 & 14 & 0 & 0 & 0 & 21 & 0 & 0 \\
\hline $35-50$ & 50 & 9 & 3 & 0 & 25 & 0 & 0 & 0 & 0 & 0 \\
\hline $50-65$ & 0 & 0 & 6 & 2 & 0 & 0 & 0 & 7 & 0 & 50 \\
\hline$>65$ & 0 & 0 & 0 & 0 & 0 & 0 & 0 & 0 & 0 & 0 \\
\hline Total & 2 & 11 & 94 & 44 & 4 & 0 & 5 & 14 & 3 & 2 \\
\hline \hline
\end{tabular}


TABLE 21: Statewide bicyclist crashes (2007-2014) by severity level, neighborhood concept and posted speed limit, continued

\begin{tabular}{c|c|c|c|c|c|c|c|c|c|c|}
\hline \hline \multirow{2}{*}{$\begin{array}{c}\text { PSL } \\
(\mathbf{m p h})\end{array}$} & \multicolumn{10}{c|}{ Neighborhood Concept D } \\
\cline { 2 - 12 } & \multicolumn{9}{|c|}{ Intersection (\%) } & \multicolumn{7}{c|}{ Segment (\%) } \\
\cline { 2 - 11 } & K & A & B & C & O & K & A & B & C & O \\
\hline NA & 11 & 52 & 57 & 60 & 77 & 29 & 41 & 55 & 57 & 92 \\
\hline$<20$ & 0 & 2 & 2 & 1 & 3 & 0 & 3 & 1 & 3 & 0 \\
\hline $20-35$ & 44 & 26 & 23 & 22 & 10 & 14 & 19 & 20 & 20 & 0 \\
\hline $35-50$ & 44 & 18 & 15 & 14 & 8 & 43 & 34 & 17 & 17 & 0 \\
\hline $50-65$ & 0 & 2 & 3 & 3 & 2 & 14 & 3 & 6 & 3 & 8 \\
\hline$>65$ & 0 & 0 & 0 & 0 & 0 & 0 & 0 & 0 & 0 & 0 \\
\hline Total & 9 & 145 & 1288 & 721 & 98 & 7 & 32 & 186 & 92 & 12 \\
\hline \hline
\end{tabular}

\begin{tabular}{c|c|c|c|c|c|c|c|c|c|c|}
\hline \hline \multirow{2}{*}{$\begin{array}{c}\text { PSL } \\
(\mathbf{m p h})\end{array}$} & \multicolumn{10}{c|}{ Neighborhood Concept E } \\
\cline { 2 - 12 } & \multicolumn{9}{|c|}{ Intersection (\%) } & \multicolumn{7}{c|}{ Segment (\%) } \\
\cline { 2 - 12 } & K & A & B & C & O & K & A & B & C & O \\
\hline NA & 14 & 35 & 44 & 47 & 72 & 14 & 35 & 41 & 39 & 60 \\
\hline$<20$ & 0 & 2 & 1 & 2 & 6 & 0 & 6 & 2 & 0 & 7 \\
\hline $20-35$ & 21 & 26 & 24 & 21 & 7 & 0 & 16 & 22 & 31 & 27 \\
\hline $35-50$ & 64 & 30 & 28 & 28 & 15 & 36 & 29 & 28 & 22 & 0 \\
\hline $50-65$ & 0 & 7 & 3 & 2 & 0 & 43 & 13 & 8 & 8 & 7 \\
\hline$>65$ & 0 & 0 & 0 & 0 & 0 & 7 & 0 & 0 & 0 & 0 \\
\hline Total & 14 & 131 & 937 & 502 & 67 & 14 & 31 & 190 & 83 & 15 \\
\hline \hline
\end{tabular}

\begin{tabular}{c|c|c|c|c|c|c|c|c|c|c|}
\hline \hline \multirow{2}{*}{$\begin{array}{c}\text { PSL } \\
(\mathbf{m p h})\end{array}$} & \multicolumn{10}{c|}{ Neighborhood Concept F } \\
\cline { 2 - 12 } & \multicolumn{9}{|c|}{ Intersection (\%) } & \multicolumn{7}{c|}{ Segment (\%) } \\
\cline { 2 - 12 } & K & A & B & C & O & K & A & B & C & O \\
\hline NA & 40 & 28 & 45 & 48 & 76 & 33 & 40 & 44 & 48 & 50 \\
\hline$<20$ & 0 & 3 & 1 & 2 & 6 & 0 & 0 & 2 & 0 & 0 \\
\hline $20-35$ & 20 & 25 & 26 & 23 & 0 & 17 & 13 & 15 & 10 & 0 \\
\hline $35-50$ & 0 & 36 & 23 & 22 & 12 & 33 & 27 & 21 & 26 & 0 \\
\hline $50-65$ & 40 & 6 & 5 & 5 & 6 & 17 & 20 & 18 & 16 & 50 \\
\hline$>65$ & 0 & 3 & 0 & 0 & 0 & 0 & 0 & 0 & 0 & 0 \\
\hline Total & 5 & 36 & 379 & 198 & 17 & 6 & 15 & 124 & 31 & 2 \\
\hline \hline
\end{tabular}

Number of lanes was assessed only for segments. Most of the fatal pedestrian crashes occurred on roads with 4 lanes. For neighborhood concept F, 2-lane roads had the highest number of crashes. This can be explained by the fact that these types of roads are mainly 
located in rural areas. Furthermore, most of the crashes that resulted in injury level, A, B,

$\mathrm{C}$ and $\mathrm{O}$, were located on 2-lane roads.

TABLE 22: Statewide pedestrian crashes (2007-2014) by severity level, neighborhood concept and number of lanes

\begin{tabular}{c|c|c|c|c|c|}
\hline \hline & \multicolumn{5}{|c|}{ Neighborhood Concept C (\%) } \\
\hline Number of lanes & K & A & B & C & O \\
\hline 0 & 0 & 0 & 0 & 0 & 0 \\
\hline 1 & 0 & 0 & 0 & 0 & 0 \\
\hline 2 & 0 & 100 & 31 & 33 & 0 \\
\hline 3 & 0 & 0 & 8 & 22 & 0 \\
\hline 4 & 0 & 0 & 38 & 33 & 0 \\
\hline 5 & 0 & 0 & 15 & 0 & 0 \\
\hline 6 & 0 & 0 & 8 & 11 & 0 \\
\hline 7 & 0 & 0 & 0 & 0 & 0 \\
\hline 8 & 0 & 0 & 0 & 0 & 0 \\
\hline Total & 0 & 1 & 13 & 9 & 0 \\
\hline \hline
\end{tabular}

\begin{tabular}{|c|c|c|c|c|c|}
\hline \hline & \multicolumn{5}{|c|}{ Neighborhood Concept D (\%) } \\
\hline Number of lanes & K & A & B & C & O \\
\hline 0 & 0 & 0 & 0 & 0 & 0 \\
\hline 1 & 0 & 1 & 0 & 0 & 0 \\
\hline 2 & 27 & 40 & 49 & 58 & 60 \\
\hline 3 & 4 & 4 & 5 & 4 & 0 \\
\hline 4 & 60 & 54 & 42 & 32 & 40 \\
\hline 5 & 4 & 2 & 3 & 6 & 0 \\
\hline 6 & 4 & 0 & 1 & 0 & 0 \\
\hline 7 & 0 & 0 & 0 & 0 & 0 \\
\hline 8 & 0 & 0 & 0 & 0 & 0 \\
\hline Total & 45 & 111 & 298 & 159 & 5 \\
\hline \hline
\end{tabular}


TABLE 22: Statewide pedestrian crashes (2007-2014) by severity level, neighborhood concept and number of lanes, continued

\begin{tabular}{c|c|c|c|c|c|}
\hline \hline & \multicolumn{5}{|c|}{ Neighborhood Concept E (\%) } \\
\hline Number of lanes & K & A & B & C & O \\
\hline 0 & 0 & 1 & 0 & 0 & 0 \\
\hline 1 & 0 & 0 & 0 & 1 & 0 \\
\hline 2 & 44 & 51 & 60 & 69 & 50 \\
\hline 3 & 3 & 1 & 3 & 2 & 20 \\
\hline 4 & 45 & 43 & 33 & 21 & 20 \\
\hline 5 & 4 & 3 & 2 & 4 & 0 \\
\hline 6 & 3 & 1 & 1 & 2 & 10 \\
\hline 7 & 0 & 0 & 0 & 1 & 0 \\
\hline 8 & 0 & 0 & 0 & 1 & 0 \\
\hline \hline Total & 117 & 148 & 345 & 193 & 10 \\
\hline
\end{tabular}

\begin{tabular}{c|c|c|c|c|c|}
\hline \hline & \multicolumn{5}{|c|}{ Neighborhood Concept F (\%) } \\
\hline Number of lanes & K & A & B & C & O \\
\hline 0 & 0 & 0 & 0 & 0 & 0 \\
\hline 1 & 0 & 0 & 1 & 0 & 0 \\
\hline 2 & 54 & 74 & 72 & 66 & 100 \\
\hline 3 & 2 & 0 & 2 & 1 & 0 \\
\hline 4 & 39 & 20 & 22 & 27 & 0 \\
\hline 5 & 0 & 3 & 2 & 1 & 0 \\
\hline 6 & 5 & 3 & 2 & 4 & 0 \\
\hline 7 & 0 & 0 & 0 & 0 & 0 \\
\hline 8 & 0 & 0 & 0 & 0 & 0 \\
\hline Total & 57 & 65 & 175 & 67 & 2 \\
\hline \hline
\end{tabular}

Most of the bicyclist crashes occurred on segments with two lanes, regardless the severity level. This finding was consistent for the different neighborhood concepts but concept D. In this land use typology, fatal crashes were mainly located at intersections with a 4-lane road. 
TABLE 23: Statewide bicyclist crashes (2007-2014) by severity level, neighborhood concept and number of lanes.

\begin{tabular}{|c|c|c|c|c|c|}
\hline \hline & \multicolumn{5}{|c|}{ Neighborhood Concept C (\%) } \\
\hline Number of lanes & K & A & B & C & O \\
\hline 0 & 0 & 0 & 0 & 0 & 0 \\
\hline 1 & 0 & 0 & 0 & 33 & 0 \\
\hline 2 & 0 & 60 & 36 & 33 & 50 \\
\hline 3 & 0 & 40 & 36 & 0 & 50 \\
\hline 4 & 0 & 0 & 29 & 33 & 0 \\
\hline 5 & 0 & 0 & 0 & 0 & 0 \\
\hline 6 & 0 & 0 & 0 & 0 & 0 \\
\hline 7 & 0 & 0 & 0 & 0 & 0 \\
\hline 8 & 0 & 100 & 100 & 100 & 100 \\
\hline Total & 0 & 5 & 14 & 3 & 2 \\
\hline \hline
\end{tabular}

\begin{tabular}{c|c|c|c|c|c|}
\hline \hline & \multicolumn{5}{|c|}{ Neighborhood Concept D (\%) } \\
\hline Number of lanes & K & A & B & C & O \\
\hline 0 & 0 & 0 & 1 & 0 & 0 \\
\hline 1 & 0 & 0 & 0 & 0 & 0 \\
\hline 2 & 29 & 69 & 60 & 62 & 29 \\
\hline 3 & 0 & 0 & 8 & 9 & 0 \\
\hline 4 & 43 & 25 & 31 & 27 & 43 \\
\hline 5 & 14 & 3 & 1 & 2 & 14 \\
\hline 6 & 14 & 3 & 0 & 0 & 14 \\
\hline 7 & 0 & 0 & 0 & 0 & 0 \\
\hline 8 & 0 & 0 & 1 & 0 & 0 \\
\hline Total & 7 & 32 & 186 & 92 & 2 \\
\hline \hline
\end{tabular}


TABLE 23: Statewide bicyclist crashes (2007-2014) by severity level, neighborhood concept and number of lanes, continued

\begin{tabular}{c|c|c|c|c|c|}
\hline \hline & \multicolumn{5}{|c|}{ Neighborhood Concept E (\%) } \\
\hline Number of lanes & K & A & B & C & O \\
\hline 0 & 0 & 0 & 0 & 0 & 0 \\
\hline 1 & 0 & 0 & 1 & 0 & 0 \\
\hline 2 & 64 & 77 & 62 & 69 & 64 \\
\hline 3 & 7 & 6 & 5 & 1 & 7 \\
\hline 4 & 21 & 16 & 25 & 28 & 21 \\
\hline 5 & 7 & 0 & 6 & 2 & 7 \\
\hline 6 & 0 & 0 & 1 & 0 & 0 \\
\hline 7 & 0 & 0 & 1 & 0 & 0 \\
\hline \hline Total & 0 & 0 & 0 & 0 & 0 \\
\hline \hline & 14 & 31 & 190 & 83 & 15 \\
\hline
\end{tabular}

\begin{tabular}{c|c|c|c|c|c|}
\hline \hline & \multicolumn{5}{|c|}{ Neighborhood Concept F (\%) } \\
\hline Number of lanes & K & A & B & C & O \\
\hline 0 & 0 & 0 & 0 & 0 & 0 \\
\hline 1 & 0 & 0 & 2 & 3 & 0 \\
\hline 2 & 83 & 80 & 77 & 74 & 50 \\
\hline 3 & 0 & 0 & 5 & 10 & 0 \\
\hline 4 & 17 & 20 & 15 & 13 & 50 \\
\hline 5 & 0 & 0 & 2 & 0 & 0 \\
\hline 6 & 0 & 0 & 0 & 0 & 0 \\
\hline 7 & 0 & 0 & 0 & 0 & 0 \\
\hline 8 & 0 & 0 & 0 & 0 & 0 \\
\hline \hline Total & 6 & 15 & 124 & 31 & 2 \\
\hline
\end{tabular}

In terms of road surface, there was not a substantial difference between the different severity levels and neighborhood concepts. Most of the pedestrian crashes occurred during dry conditions. Furthermore, the second most important road surface category was wet, regardless of the severity level. 
TABLE 24: Statewide pedestrian crashes (2007-2014) by severity level, neighborhood concept and road surface

\begin{tabular}{c|c|c|c|c|c|c|c|c|c|c|}
\hline \hline \multirow{2}{*}{$\begin{array}{c}\text { Road } \\
\text { surface }\end{array}$} & \multicolumn{10}{c|}{ Neighborhood Concept C } \\
\cline { 2 - 12 } & \multicolumn{9}{|c|}{ Intersection (\%) } & \multicolumn{7}{c|}{ Segment (\%) } \\
\cline { 2 - 12 } & K & A & B & C & O & K & A & B & C & O \\
\hline Unknown & 0 & 0 & 1 & 4 & 0 & 0 & 0 & 0 & 0 & 0 \\
\hline Dry & 100 & 86 & 59 & 50 & 100 & 0 & 100 & 85 & 44 & 0 \\
\hline Wet & 0 & 14 & 40 & 44 & 0 & 0 & 0 & 15 & 44 & 0 \\
\hline Other & 0 & 0 & 0 & 1 & 0 & 0 & 0 & 0 & 11 & 0 \\
\hline Total & 1 & 14 & 81 & 68 & 1 & 0 & 1 & 13 & 9 & 0 \\
\hline \hline
\end{tabular}

\begin{tabular}{c|c|c|c|c|c|c|c|c|c|c|}
\hline \hline \multirow{2}{*}{$\begin{array}{c}\text { Road } \\
\text { surface }\end{array}$} & \multicolumn{10}{c|}{ Neighborhood Concept D } \\
\cline { 2 - 12 } & \multicolumn{1}{|c|}{ Intersection (\%) } & \multicolumn{7}{c|}{ Segment (\%) } \\
\cline { 2 - 12 } & K & A & B & C & O & K & A & B & C & O \\
\hline Unknown & 6 & 1 & 1 & 1 & 13 & 2 & 0 & 0 & 2 & 0 \\
\hline Dry & 70 & 73 & 68 & 60 & 56 & 72 & 75 & 74 & 69 & 80 \\
\hline Wet & 24 & 25 & 30 & 38 & 31 & 26 & 23 & 25 & 29 & 20 \\
\hline Other & 0 & 1 & 1 & 1 & 0 & 0 & 2 & 0 & 0 & 0 \\
\hline Total & 54 & 173 & 852 & 638 & 16 & 46 & 111 & 298 & 160 & 5 \\
\hline \hline
\end{tabular}

\begin{tabular}{c|c|c|c|c|c|c|c|c|c|c|}
\hline \hline \multirow{2}{*}{$\begin{array}{c}\text { Road } \\
\text { surface }\end{array}$} & \multicolumn{10}{c|}{ Neighborhood Concept E } \\
\cline { 2 - 12 } & \multicolumn{9}{|c|}{ Intersection (\%) } & \multicolumn{5}{c|}{ Segment (\%) } \\
\cline { 2 - 11 } & K & A & B & C & O & K & A & B & C & O \\
\hline Unknown & 0 & 1 & 1 & 2 & 13 & 9 & 1 & 1 & 1 & 0 \\
\hline Dry & 67 & 63 & 71 & 62 & 69 & 60 & 70 & 70 & 71 & 100 \\
\hline Wet & 31 & 32 & 26 & 35 & 19 & 32 & 28 & 27 & 25 & 0 \\
\hline Other & 2 & 4 & 1 & 2 & 0 & 0 & 2 & 2 & 3 & 0 \\
\hline Total & 48 & 146 & 588 & 471 & 16 & 117 & 148 & 345 & 193 & 10 \\
\hline \hline
\end{tabular}

\begin{tabular}{c|c|c|c|c|c|c|c|c|c|c|}
\hline \hline \multirow{2}{*}{$\begin{array}{c}\text { Road } \\
\text { surface }\end{array}$} & \multicolumn{10}{c|}{ Neighborhood Concept F } \\
\cline { 2 - 12 } & \multicolumn{1}{|c|}{ Intersection (\%) } & \multicolumn{7}{c|}{ Segment (\%) } \\
\cline { 2 - 12 } & K & A & B & C & O & K & A & B & C & O \\
\hline Unknown & 0 & 0 & 0 & 2 & 0 & 4 & 3 & 1 & 3 & 0 \\
\hline Dry & 81 & 62 & 69 & 62 & 43 & 67 & 78 & 73 & 73 & 50 \\
\hline Wet & 19 & 36 & 29 & 34 & 57 & 28 & 17 & 21 & 15 & 0 \\
\hline Other & 0 & 2 & 2 & 2 & 0 & 2 & 2 & 6 & 9 & 50 \\
\hline Total & 16 & 53 & 230 & 156 & 7 & 57 & 65 & 175 & 67 & 2 \\
\hline \hline
\end{tabular}


Similar results were found for bicyclist crashes. Most of the observations were concentrated on dry road surfaces, regardless of the severity level.

TABLE 25: Statewide bicyclist crashes (2007-2014) by severity level, neighborhood concept and road surface

\begin{tabular}{c|c|c|c|c|c|c|c|c|c|c|}
\hline \hline \multirow{2}{*}{$\begin{array}{c}\text { Road } \\
\text { surface }\end{array}$} & \multicolumn{10}{c|}{ Neighborhood Concept C } \\
\cline { 2 - 11 } & \multicolumn{9}{|c|}{ Intersection (\%) } & \multicolumn{7}{c|}{ Segment (\%) } \\
\cline { 2 - 11 } & K & A & B & C & O & K & A & B & C & O \\
\hline Unknown & 0 & 0 & 1 & 2 & 0 & 0 & 20 & 0 & 0 & 0 \\
\hline Dry & 50 & 100 & 79 & 80 & 75 & 0 & 40 & 79 & 100 & 50 \\
\hline Wet & 50 & 0 & 20 & 18 & 25 & 0 & 40 & 21 & 0 & 50 \\
\hline Other & 0 & 0 & 0 & 0 & 0 & 0 & 0 & 0 & 0 & 0 \\
\hline Total & 2 & 11 & 94 & 44 & 4 & 0 & 5 & 14 & 3 & 2 \\
\hline \hline
\end{tabular}

\begin{tabular}{c|c|c|c|c|c|c|c|c|c|c|}
\hline \hline \multirow{2}{*}{$\begin{array}{c}\text { Road } \\
\text { surface }\end{array}$} & \multicolumn{10}{c|}{ Neighborhood Concept D } \\
\cline { 2 - 12 } & \multicolumn{9}{|c|}{ Intersection (\%) } & \multicolumn{5}{c|}{ Segment (\%) } \\
\cline { 2 - 12 } & K & A & B & C & O & K & A & B & C & O \\
\hline Unknown & 0 & 0 & 1 & 2 & 7 & 14 & 0 & 2 & 1 & 0 \\
\hline Dry & 100 & 82 & 85 & 76 & 72 & 71 & 88 & 89 & 80 & 83 \\
\hline Wet & 0 & 18 & 13 & 21 & 20 & 0 & 13 & 9 & 18 & 17 \\
\hline Other & 0 & 0 & 0 & 0 & 0 & 14 & 0 & 0 & 0 & 0 \\
\hline Total & 9 & 145 & 1288 & 721 & 98 & 7 & 32 & 186 & 92 & 12 \\
\hline \hline
\end{tabular}

\begin{tabular}{c|c|c|c|c|c|c|c|c|c|c|}
\hline \hline \multirow{2}{*}{$\begin{array}{c}\text { Road } \\
\text { surface }\end{array}$} & \multicolumn{10}{c|}{ Neighborhood Concept E } \\
\cline { 2 - 12 } & \multicolumn{9}{|c|}{ Intersection (\%) } & \multicolumn{5}{c|}{ Segment (\%) } \\
\cline { 2 - 12 } & K & A & B & C & O & K & A & B & C & O \\
\hline Unknown & 14 & 1 & 0 & 2 & 4 & 0 & 0 & 1 & 2 & 7 \\
\hline Dry & 64 & 84 & 87 & 77 & 81 & 71 & 94 & 92 & 87 & 87 \\
\hline Wet & 21 & 15 & 12 & 21 & 15 & 29 & 6 & 7 & 11 & 7 \\
\hline Other & 14 & 1 & 0 & 2 & 4 & 0 & 0 & 1 & 2 & 7 \\
\hline Total & 14 & 131 & 937 & 502 & 67 & 14 & 31 & 190 & 83 & 15 \\
\hline \hline
\end{tabular}


TABLE 25: Statewide bicyclist crashes (2007-2014) by severity level, neighborhood concept and road surface, continued

\begin{tabular}{c|c|c|c|c|c|c|c|c|c|c|}
\hline \hline \multirow{2}{*}{$\begin{array}{c}\text { Road } \\
\text { surface }\end{array}$} & \multicolumn{10}{c|}{ Neighborhood Concept F } \\
\cline { 2 - 11 } & \multicolumn{9}{|c|}{ Intersection (\%) } & \multicolumn{7}{c|}{ Segment (\%) } \\
\cline { 2 - 11 } & K & A & B & C & O & K & A & B & C & O \\
\hline Unknown & 0 & 0 & 1 & 3 & 6 & 0 & 7 & 3 & 3 & 0 \\
\hline Dry & 100 & 94 & 86 & 84 & 71 & 83 & 87 & 87 & 65 & 100 \\
\hline Wet & 0 & 6 & 13 & 14 & 24 & 17 & 7 & 10 & 32 & 0 \\
\hline Other & 0 & 0 & 0 & 0 & 0 & 0 & 0 & 0 & 0 & 0 \\
\hline Total & 5 & 36 & 379 & 198 & 17 & 6 & 15 & 124 & 31 & 2 \\
\hline \hline
\end{tabular}

\subsection{Environmental conditions}

For environmental conditions, the literature review found that weather, time of the day, day of the week, and light conditions were good predictors for crash severity. Most of the crashes occurred on clear days, followed by rainy days. This result was similar across the different neighborhood concepts and severity levels. This finding can be the result of less pedestrian volumes in rainy and cloudy weather conditions, which results in less exposure.

TABLE 26: Statewide pedestrian crashes (2007-2014) by severity level, neighborhood concept and weather conditions

\begin{tabular}{c|c|c|c|c|c|c|c|c|c|c|}
\hline \hline \multirow{2}{*}{ Weather } & \multicolumn{8}{|c|}{ Neighborhood Concept C } \\
\cline { 2 - 12 } & \multicolumn{9}{|c|}{ Intersection (\%) } & \multicolumn{7}{c|}{ Segment (\%) } \\
\cline { 2 - 12 } & $\mathbf{K}$ & $\mathbf{A}$ & $\mathbf{B}$ & $\mathbf{C}$ & $\mathbf{O}$ & $\mathbf{K}$ & $\mathbf{A}$ & $\mathbf{B}$ & $\mathbf{C}$ & $\mathbf{O}$ \\
\hline Unknown & 0 & 0 & 2 & 3 & 0 & 0 & 0 & 0 & 0 & 0 \\
\hline Clear & 0 & 71 & 58 & 49 & 100 & 0 & 100 & 54 & 33 & 0 \\
\hline Cloudy & 0 & 14 & 12 & 7 & 0 & 0 & 0 & 31 & 11 & 0 \\
\hline Rain & 0 & 14 & 26 & 40 & 0 & 0 & 0 & 15 & 44 & 0 \\
\hline Others & 0 & 0 & 2 & 3 & 0 & 0 & 0 & 0 & 0 & 0 \\
\hline Total & 1 & 14 & 81 & 68 & 1 & 0 & 1 & 13 & 9 & 0 \\
\hline \hline
\end{tabular}


TABLE 26: Statewide pedestrian crashes (2007-2014) by severity level, neighborhood concept and weather conditions, contiuned

\begin{tabular}{c|c|c|c|c|c|c|c|c|c|c|}
\hline \hline \multirow{3}{*}{ Weather } & \multicolumn{10}{|c|}{ Neighborhood Concept D } \\
\cline { 2 - 12 } & \multicolumn{1}{|c|}{ Intersection (\%) } & \multicolumn{7}{c|}{ Segment (\%) } \\
\cline { 2 - 12 } & $\mathbf{A}$ & $\mathbf{B}$ & $\mathbf{C}$ & $\mathbf{O}$ & $\mathbf{K}$ & $\mathbf{A}$ & $\mathbf{B}$ & $\mathbf{C}$ & $\mathbf{O}$ \\
\hline Unknown & 4 & 2 & 1 & 1 & 13 & 2 & 0 & 2 & 2 & 0 \\
\hline Clear & 52 & 58 & 57 & 53 & 56 & 43 & 51 & 63 & 56 & 60 \\
\hline Cloudy & 28 & 21 & 16 & 14 & 0 & 35 & 26 & 16 & 18 & 0 \\
\hline Rain & 15 & 18 & 23 & 30 & 31 & 15 & 19 & 18 & 21 & 20 \\
\hline Others & 2 & 1 & 2 & 2 & 0 & 4 & 4 & 1 & 3 & 20 \\
\hline Total & 54 & 173 & 852 & 638 & 16 & 46 & 111 & 299 & 164 & 5 \\
\hline \hline
\end{tabular}

\begin{tabular}{|c|c|c|c|c|c|c|c|c|c|c|}
\hline \multirow[b]{3}{*}{ Weather } & \multicolumn{10}{|c|}{ Neighborhood Concept E } \\
\hline & \multicolumn{5}{|c|}{ Intersection (\%) } & \multicolumn{5}{|c|}{ Segment $(\%)$} \\
\hline & $\mathbf{K}$ & $\mathbf{A}$ & B & $\mathrm{C}$ & $\mathbf{O}$ & $\mathbf{K}$ & A & B & $\mathbf{C}$ & $\mathbf{O}$ \\
\hline Unknown & 0 & 1 & 1 & 2 & 13 & 8 & 1 & 1 & 1 & 0 \\
\hline Clear & 56 & 45 & 62 & 49 & 44 & 38 & 53 & 57 & 63 & 90 \\
\hline Cloudy & 21 & 23 & 16 & 18 & 25 & 37 & 24 & 22 & 17 & 10 \\
\hline Rain & 21 & 29 & 19 & 27 & 13 & 16 & 20 & 17 & 18 & 0 \\
\hline Others & 2 & 3 & 3 & 3 & 6 & 2 & 1 & 3 & 2 & 0 \\
\hline Total & 48 & 146 & 586 & 471 & 16 & 117 & 147 & 345 & 193 & 10 \\
\hline
\end{tabular}

\begin{tabular}{c|c|c|c|c|c|c|c|c|c|c|}
\hline \hline \multirow{3}{*}{ Weather } & \multicolumn{10}{|c|}{ Neighborhood Concept F } \\
\cline { 2 - 12 } & \multicolumn{9}{|c|}{ Intersection (\%) } & \multicolumn{7}{c|}{ Segment (\%) } \\
\cline { 2 - 12 } & K & A & B & C & O & K & A & B & C & O \\
\hline Unknown & 13 & 6 & 6 & 8 & 0 & 4 & 2 & 1 & 4 & 0 \\
\hline Clear & 31 & 45 & 51 & 44 & 29 & 53 & 69 & 61 & 66 & 50 \\
\hline Cloudy & 38 & 17 & 21 & 19 & 29 & 16 & 12 & 16 & 19 & 0 \\
\hline Rain & 19 & 25 & 20 & 24 & 43 & 25 & 17 & 16 & 9 & 0 \\
\hline Others & 0 & 8 & 2 & 4 & 0 & 4 & 0 & 6 & 1 & 50 \\
\hline Total & 16 & 53 & 230 & 156 & 7 & 57 & 65 & 175 & 67 & 2 \\
\hline \hline
\end{tabular}

Very similar results were found for bicyclist crashes. After clear days, cloudy conditions had the second highest number of crashes regardless of the severity level. Number of crashes during rainy conditions was also low, which is expected due to the low bicyclist volume under rain events. 
TABLE 27: Statewide bicyclist crashes (2007-2014) by severity level, neighborhood concept and weather conditions

\begin{tabular}{|c|c|c|c|c|c|c|c|c|c|c|}
\hline \multirow[b]{3}{*}{ Weather } & \multicolumn{10}{|c|}{ Neighborhood Concept $\mathbf{C}$} \\
\hline & \multicolumn{5}{|c|}{ Intersection (\%) } & \multicolumn{5}{|c|}{ Segment $(\%)$} \\
\hline & $\mathbf{K}$ & $\mathbf{A}$ & B & C & $\mathbf{O}$ & $\mathbf{K}$ & $\mathbf{A}$ & B & C & $\mathbf{O}$ \\
\hline Unknown & 0 & 0 & 2 & 2 & 0 & 20 & 0 & 0 & 0 & 0 \\
\hline Clear & 50 & 100 & 71 & 73 & 75 & 40 & 64 & 67 & 100 & 0 \\
\hline Cloudy & 0 & 0 & 11 & 11 & 0 & 20 & 14 & 33 & 0 & 0 \\
\hline Rain & 50 & 0 & 15 & 14 & 25 & 20 & 14 & 0 & 0 & 0 \\
\hline Others & 0 & 0 & 1 & 0 & 0 & 0 & 7 & 0 & 0 & 0 \\
\hline Total & 2 & 11 & 94 & 44 & 4 & 5 & 14 & 3 & 2 & 0 \\
\hline
\end{tabular}

\begin{tabular}{c|c|c|c|c|c|c|c|c|c|c|}
\hline \hline & \multicolumn{10}{|c|}{ Neighborhood Concept D } \\
\cline { 2 - 12 } Weather & \multicolumn{1}{|c|}{ Intersection (\%) } & \multicolumn{7}{c|}{ Segment (\%) } \\
\cline { 2 - 12 } & K & A & B & C & O & K & A & B & C & O \\
\hline Unknown & 0 & 1 & 1 & 2 & 7 & 14 & 0 & 2 & 1 & 0 \\
\hline Clear & 56 & 72 & 77 & 67 & 76 & 29 & 81 & 80 & 76 & 75 \\
\hline Cloudy & 44 & 12 & 12 & 16 & 4 & 57 & 9 & 12 & 10 & 8 \\
\hline Rain & 0 & 14 & 9 & 14 & 13 & 0 & 9 & 6 & 12 & 17 \\
\hline Others & 0 & 1 & 0 & 1 & 0 & 0 & 0 & 0 & 1 & 0 \\
\hline Total & 9 & 145 & 1288 & 721 & 98 & 7 & 32 & 186 & 92 & 12 \\
\hline \hline
\end{tabular}

\begin{tabular}{c|c|c|c|c|c|c|c|c|c|c|}
\hline \hline \multirow{3}{*}{ Weather } & \multicolumn{10}{|c|}{ Neighborhood Concept E } \\
\cline { 2 - 12 } & \multicolumn{1}{|c|}{ Intersection (\%) } & \multicolumn{7}{c|}{ Segment (\%) } \\
\cline { 2 - 12 } & $\mathbf{K}$ & $\mathbf{A}$ & $\mathbf{B}$ & $\mathbf{C}$ & $\mathbf{O}$ & $\mathbf{K}$ & $\mathbf{A}$ & $\mathbf{B}$ & $\mathbf{C}$ & $\mathbf{O}$ \\
\hline Unknown & 14 & 2 & 1 & 1 & 3 & 0 & 0 & 2 & 2 & 7 \\
\hline Clear & 50 & 76 & 76 & 68 & 79 & 50 & 81 & 79 & 80 & 73 \\
\hline Cloudy & 21 & 15 & 15 & 15 & 10 & 43 & 13 & 13 & 10 & 20 \\
\hline Rain & 14 & 8 & 8 & 14 & 7 & 7 & 6 & 5 & 8 & 0 \\
\hline Others & 0 & 0 & 1 & 1 & 0 & 0 & 0 & 1 & 0 & 0 \\
\hline Total & 14 & 131 & 937 & 502 & 67 & 14 & 31 & 190 & 83 & 15 \\
\hline \hline
\end{tabular}


TABLE 27: Statewide bicyclist crashes (2007-2014) by severity level, neighborhood concept and weather conditions, contiuned

\begin{tabular}{c|c|c|c|c|c|c|c|c|c|c|}
\hline \multirow{2}{*}{ Weather } & \multicolumn{10}{c|}{ Neighborhood Concept F } \\
\cline { 2 - 12 } & \multicolumn{1}{|c|}{ Intersection (\%) } & \multicolumn{7}{c|}{ Segment (\%) } \\
\cline { 2 - 12 } & K & A & B & C & O & K & A & B & C & O \\
\hline Unknown & 0 & 3 & 1 & 3 & 6 & 0 & 0 & 3 & 3 & 0 \\
\hline Clear & 60 & 81 & 75 & 71 & 76 & 83 & 93 & 75 & 48 & 100 \\
\hline Cloudy & 40 & 8 & 13 & 16 & 6 & 0 & 7 & 16 & 26 & 0 \\
\hline Rain & 0 & 6 & 11 & 10 & 12 & 0 & 0 & 4 & 19 & 0 \\
\hline Others & 0 & 3 & 1 & 1 & 0 & 17 & 0 & 2 & 3 & 0 \\
\hline Total & 5 & 36 & 379 & 198 & 17 & 6 & 15 & 124 & 31 & 2 \\
\hline \hline
\end{tabular}

At intersections, the majority of crashes occurred during daylight conditions.

Furthermore, the descriptive analysis revealed that fatal crashes for concept E occurred mainly under dark environments with street light. For segments of the road, most of the fatal crashes (injury level A was substantial as well) occurred under dark conditions with no streetlight (except for concept D, where the highest number of fatal crashes occurred on dark conditions with streetlight).

TABLE 28: Statewide pedestrian crashes (2007-2014) by severity level, neighborhood concept and light conditions

\begin{tabular}{c|c|c|c|c|c|c|c|c|c|c|}
\hline \hline \multirow{2}{*}{$\begin{array}{c}\text { Light } \\
\text { conditions }\end{array}$} & \multicolumn{10}{c|}{ Neighborhood Concept C } \\
\cline { 2 - 12 } & \multicolumn{9}{|c|}{ Intersection (\%) } & \multicolumn{7}{c|}{ Segment (\%) } \\
\cline { 2 - 12 } & K & A & B & C & O & K & A & B & C & O \\
\hline Unknown & 0 & 0 & 0 & 0 & 0 & 0 & 0 & 0 & 0 & 0 \\
\hline Daylight & 0 & 100 & 67 & 68 & 100 & 0 & 0 & 31 & 44 & 0 \\
\hline $\begin{array}{c}\text { Darkness- } \\
\text { with street } \\
\text { light }\end{array}$ & 50 & 0 & 23 & 23 & 0 & & 100 & 62 & 44 & 0 \\
\hline $\begin{array}{c}\text { Darkness- } \\
\text { no street } \\
\text { light }\end{array}$ & 0 & 0 & 2 & 2 & 0 & & 0 & 0 & 11 & 0 \\
\hline Dawn & 0 & 0 & 3 & 7 & 0 & 0 & 0 & 0 & 0 & 0 \\
\hline Dusk & 50 & 0 & 4 & 0 & 0 & 0 & 0 & 8 & 0 & 0 \\
\hline Total & 2 & 11 & 94 & 44 & 4 & 0 & 1 & 13 & 9 & 0 \\
\hline \hline
\end{tabular}


TABLE 28: Statewide pedestrian crashes (2007-2014) by severity level, neighborhood concept and light conditions, continued

\begin{tabular}{c|c|c|c|c|c|c|c|c|c|c|}
\hline \hline \multirow{2}{*}{$\begin{array}{c}\text { Light } \\
\text { conditions }\end{array}$} & \multicolumn{9}{|c|}{ Neighborhood Concept D } \\
\cline { 2 - 12 } & \multicolumn{9}{|c|}{ Intersection (\%) } & \multicolumn{7}{c|}{ Segment (\%) } \\
\cline { 2 - 12 } & K & A & B & C & O & K & A & B & C & O \\
\hline Unknown & 2 & 0 & 0 & 0 & 6 & 0 & 0 & 0 & 0 & 0 \\
\hline Daylight & 44 & 49 & 58 & 55 & 75 & 20 & 34 & 49 & 56 & 60 \\
\hline $\begin{array}{c}\text { Darkness- } \\
\text { with street } \\
\text { light }\end{array}$ & 43 & 33 & 30 & 30 & 19 & 57 & 40 & 35 & 28 & 40 \\
\hline $\begin{array}{c}\text { Darkness- } \\
\text { no street } \\
\text { light }\end{array}$ & 11 & 6 & 5 & 6 & 0 & 17 & 15 & 7 & 9 & 0 \\
\hline Dawn & 0 & 5 & 2 & 3 & 0 & 0 & 3 & 2 & 1 & 0 \\
\hline Dusk & 0 & 8 & 5 & 5 & 0 & 7 & 8 & 6 & 7 & 0 \\
\hline Total & 54 & 173 & 852 & 638 & 16 & 46 & 111 & 298 & 160 & 5 \\
\hline \hline
\end{tabular}

\begin{tabular}{c|c|c|c|c|c|c|c|c|c|c|}
\hline \hline \multirow{2}{*}{$\begin{array}{c}\text { Light } \\
\text { conditions }\end{array}$} & \multicolumn{10}{c|}{ Neighborhood Concept E } \\
\cline { 2 - 12 } & \multicolumn{9}{|c|}{ Intersection (\%) } & \multicolumn{7}{c|}{ Segment (\%) } \\
\cline { 2 - 12 } & K & A & B & C & O & K & A & B & C & O \\
\hline Unknown & 0 & 0 & 0 & 0 & 0 & 2 & 0 & 0 & 0 & 0 \\
\hline Daylight & 33 & 49 & 64 & 54 & 63 & 16 & 39 & 53 & 51 & 70 \\
\hline $\begin{array}{c}\text { Darkness- } \\
\text { with street } \\
\text { light }\end{array}$ & 46 & 28 & 22 & 28 & 19 & 24 & 21 & 19 & 23 & 10 \\
\hline $\begin{array}{c}\text { Darkness- } \\
\text { no street } \\
\text { light }\end{array}$ & 15 & 12 & 5 & 9 & 19 & 50 & 27 & 19 & 20 & 0 \\
\hline Dawn & 2 & 5 & 3 & 3 & 0 & 1 & 1 & 1 & 1 & 0 \\
\hline Dusk & 4 & 7 & 5 & 5 & 0 & 7 & 11 & 7 & 5 & 20 \\
\hline Total & 48 & 146 & 588 & 471 & 16 & 117 & 148 & 345 & 193 & 10 \\
\hline \hline
\end{tabular}


TABLE 28: Statewide pedestrian crashes (2007-2014) by severity level, neighborhood concept and light conditions, continued

\begin{tabular}{c|c|c|c|c|c|c|c|c|c|c|}
\hline \hline \multirow{2}{*}{$\begin{array}{c}\text { Light } \\
\text { conditions }\end{array}$} & \multicolumn{10}{c|}{ Neighborhood Concept F } \\
\cline { 2 - 12 } & \multicolumn{9}{|c|}{ Intersection (\%) } & \multicolumn{7}{c|}{ Segment (\%) } \\
\cline { 2 - 12 } & K & A & B & C & O & K & A & B & C & O \\
\hline Unknown & 0 & 0 & 0 & 1 & 0 & 0 & 2 & 0 & 0 & 0 \\
\hline Daylight & 44 & 53 & 60 & 52 & 71 & 16 & 42 & 55 & 43 & 100 \\
\hline $\begin{array}{c}\text { Darkness- } \\
\text { with street } \\
\text { light }\end{array}$ & 31 & 21 & 25 & 28 & 29 & 16 & 14 & 12 & 27 & 0 \\
\hline $\begin{array}{c}\text { Darkness- } \\
\text { no street } \\
\text { light }\end{array}$ & 25 & 19 & 8 & 8 & 0 & 56 & 32 & 22 & 22 & 0 \\
\hline Dawn & 0 & 4 & 2 & 7 & 0 & 5 & 6 & 4 & 1 & 0 \\
\hline Dusk & 0 & 4 & 5 & 3 & 0 & 7 & 5 & 6 & 6 & 0 \\
\hline Total & 16 & 53 & 230 & 156 & 7 & 57 & 65 & 175 & 67 & 2 \\
\hline \hline
\end{tabular}

For bicyclist crashes, the trend was not as visible as for pedestrian crashes. Most of the crashes occurred during daylight conditions regardless the severity level. Nonetheless, it was followed by dark conditions with no street light. There was less bicyclist activity during the night since the commuting time is over and bicycle recreational activities did not tend to occur at night. 
TABLE 29: Statewide bicyclist crashes (2007-2014) by severity level, neighborhood concept and light conditions

\begin{tabular}{c|c|c|c|c|c|c|c|c|c|c|}
\hline \hline \multirow{2}{*}{$\begin{array}{c}\text { Light } \\
\text { conditions }\end{array}$} & \multicolumn{10}{c|}{ Neighborhood Concept C } \\
\cline { 2 - 12 } & \multicolumn{9}{|c|}{ Intersection (\%) } & \multicolumn{7}{c|}{ Segment (\%) } \\
\cline { 2 - 12 } & K & A & B & C & O & K & A & B & C & O \\
\hline Unknown & 0 & 0 & 0 & 0 & 0 & 0 & 0 & 0 & 0 & 0 \\
\hline Daylight & 0 & 100 & 67 & 68 & 100 & 0 & 40 & 79 & 67 & 50 \\
\hline $\begin{array}{c}\text { Darkness- } \\
\text { with street } \\
\text { light }\end{array}$ & 50 & 0 & 23 & 23 & 0 & & 40 & 21 & 33 & 50 \\
\hline $\begin{array}{c}\text { Darkness- } \\
\text { no street } \\
\text { light }\end{array}$ & 0 & 0 & 2 & 2 & 0 & & 0 & 0 & 0 & 0 \\
\hline Dawn & 0 & 0 & 3 & 7 & 0 & 0 & 0 & 0 & 0 & 0 \\
\hline Dusk & 50 & 0 & 4 & 0 & 0 & 0 & 20 & 0 & 0 & 0 \\
\hline Total & 2 & 11 & 94 & 44 & 4 & 0 & 5 & 14 & 3 & 2 \\
\hline \hline
\end{tabular}

\begin{tabular}{c|c|c|c|c|c|c|c|c|c|c|}
\hline \hline \multirow{2}{*}{$\begin{array}{c}\text { Light } \\
\text { conditions }\end{array}$} & \multicolumn{10}{c|}{ Neighborhood Concept D } \\
\cline { 2 - 12 } & \multicolumn{9}{|c|}{ Intersection (\%) } & \multicolumn{7}{c|}{ Segment (\%) } \\
\cline { 2 - 12 } & K & A & B & C & O & K & A & B & C & O \\
\hline Unknown & 2 & 0 & 0 & 0 & 6 & 0 & 0 & 0 & 0 & 0 \\
\hline Daylight & 44 & 49 & 58 & 55 & 75 & 20 & 34 & 49 & 56 & 60 \\
\hline $\begin{array}{c}\text { Darkness- } \\
\text { with street } \\
\text { light }\end{array}$ & 43 & 33 & 30 & 30 & 19 & 57 & 40 & 35 & 28 & 40 \\
\hline $\begin{array}{c}\text { Darkness- } \\
\text { no street } \\
\text { light }\end{array}$ & 11 & 6 & 5 & 6 & 0 & 17 & 15 & 7 & 9 & 0 \\
\hline Dawn & 0 & 5 & 2 & 3 & 0 & 0 & 3 & 2 & 1 & 0 \\
\hline Dusk & 0 & 8 & 5 & 5 & 0 & 7 & 8 & 6 & 7 & 0 \\
\hline Total & 54 & 173 & 852 & 638 & 16 & 46 & 111 & 298 & 160 & 5 \\
\hline \hline
\end{tabular}


TABLE 29: Statewide bicyclist crashes (2007-2014) by severity level, neighborhood concept and light conditions, continued

\begin{tabular}{c|c|c|c|c|c|c|c|c|c|c|}
\hline \hline \multirow{2}{*}{$\begin{array}{c}\text { Light } \\
\text { conditions }\end{array}$} & \multicolumn{10}{c|}{ Neighborhood Concept E } \\
\cline { 2 - 12 } & \multicolumn{9}{|c|}{ Intersection (\%) } & \multicolumn{7}{c|}{ Segment (\%) } \\
\cline { 2 - 12 } & K & A & B & C & O & K & A & B & C & O \\
\hline Unknown & 0 & 0 & 0 & 0 & 0 & 0 & 0 & 0 & 0 & 0 \\
\hline Daylight & 64 & 82 & 81 & 76 & 78 & 57 & 90 & 80 & 81 & 87 \\
\hline $\begin{array}{c}\text { Darkness- } \\
\text { with street } \\
\text { light }\end{array}$ & 21 & 11 & 9 & 11 & 12 & 7 & 0 & 10 & 5 & 7 \\
\hline $\begin{array}{c}\text { Darkness- } \\
\text { no street } \\
\text { light }\end{array}$ & 0 & 5 & 2 & 4 & 0 & 29 & 6 & 5 & 6 & 0 \\
\hline Dawn & 14 & 0 & 3 & 4 & 6 & 0 & 3 & 1 & 4 & 7 \\
\hline Dusk & 0 & 3 & 5 & 5 & 4 & 7 & 0 & 4 & 5 & 0 \\
\hline Total & 14 & 131 & 937 & 502 & 67 & 14 & 31 & 190 & 83 & 15 \\
\hline \hline
\end{tabular}

\begin{tabular}{c|c|c|c|c|c|c|c|c|c|c|}
\hline \hline \multirow{2}{*}{$\begin{array}{c}\text { Light } \\
\text { conditions }\end{array}$} & \multicolumn{10}{c|}{ Neighborhood Concept F } \\
\cline { 2 - 12 } & \multicolumn{9}{|c|}{ Intersection (\%) } & \multicolumn{7}{c|}{ Segment (\%) } \\
\cline { 2 - 12 } & K & A & B & C & O & K & A & B & C & O \\
\hline Unknown & 0 & 2 & 0 & 1 & 0 & 0 & 0 & 0 & 0 & 0 \\
\hline Daylight & 83 & 75 & 79 & 74 & 75 & 67 & 73 & 77 & 68 & 50 \\
\hline $\begin{array}{c}\text { Darkness- } \\
\text { with street } \\
\text { light }\end{array}$ & 0 & 14 & 10 & 13 & 0 & 0 & 0 & 7 & 6 & 50 \\
\hline $\begin{array}{c}\text { Darkness- } \\
\text { no street } \\
\text { light }\end{array}$ & 17 & 6 & 3 & 4 & 13 & 33 & 13 & 6 & 16 & 0 \\
\hline Dawn & 0 & 2 & 3 & 3 & 6 & 0 & 0 & 3 & 3 & 0 \\
\hline Dusk & 0 & 2 & 5 & 5 & 6 & 0 & 13 & 6 & 6 & 0 \\
\hline Total & 6 & 64 & 366 & 183 & 16 & 6 & 15 & 124 & 31 & 2 \\
\hline \hline
\end{tabular}

\subsection{Summary}

This initial effort to examine the data helped us exploring risk factors associated to crash severity in state roads, and provided us with a preliminary understanding of how severity levels were affected by exposure. The descriptive analysis suggested that age plays an 
important role in crash severity. In terms of road characteristics, arterials, 4 lane roads (2 lane roads for bicyclist crashes), and high-posted speed limit roads were potential risk factors for pedestrian and bicyclist crash severity. In terms of exposure, we started to explore neighborhood concepts as a surrogate of pedestrian and bicyclist activity levels and exposure. We found that most of the crashes occurred on concept E and F, or suburban and rural. 


\section{EXPOSURE ANALYSIS}

One of the most challenging tasks in safety research is the analysis of crash rates but accounting for exposure. Since detailed pedestrian and bicyclist volumes are typically not available, it is necessary to account for exposure utilizing indirect methods. We have tried to control for exposure in this chapter by analyzing the impact of (VMT) and pedestrian and bicyclist volumes (by using a neighborhood concepts as a surrogate). The analysis was limited to the Oregon State Network (TransGIS database) only because it did include complete records for AADT, posted speed limit, the number of lanes, road classification and road width.

TABLE 30: Pedestrian and bicyclist crashes in Oregon

\begin{tabular}{c|c|c|c|c}
\hline \hline & $\begin{array}{c}\text { PEDESTRIAN } \\
\text { CRASHES IN } \\
\text { OREGON }\end{array}$ & $\begin{array}{c}\text { PEDESTRIAN } \\
\text { CRASHES IN } \\
\text { OREGON } \\
\text { STATE } \\
\text { HIGHWAYS } \\
\text { (only at } \\
\text { segments and } \\
\text { intersections) }\end{array}$ & $\begin{array}{c}\text { BICYCLIST } \\
\text { CRASHES IN } \\
\text { OREGON }\end{array}$ & $\begin{array}{c}\text { BICYCIST } \\
\text { CRASHES IN } \\
\text { OREGON } \\
\text { STATE } \\
\text { HIGHWAYS } \\
\text { (only at } \\
\text { segments and } \\
\text { intersections) }\end{array}$ \\
\hline Total crashes & 6,162 & 1,840 & 7,147 & 1,584 \\
\hline $\begin{array}{c}\text { Crashes at } \\
\text { intersections }\end{array}$ & 3,629 & 1,088 & 4,702 & 1,045 \\
\hline $\begin{array}{c}\text { Crashes at } \\
\text { segments }\end{array}$ & 1,822 & 561 & 864 & 169 \\
\hline Others & 711 & 191 & 1481 & 370 \\
\hline \hline
\end{tabular}

The following analysis considered the percentage of crashes that took place in the Oregon State Highway Network. Crashes were categorized by posted speed limit, number of lanes, road width, and road classification. Exposure was controlled by estimating the proportion of the Oregon State Highway Network VMT (using pedestrian crashes per 10,000 AADT and highway length). 
The following tables show the ratio of the percentage of crashes to the percentage of the Oregon State Highway Network length by category (e.g. AADT range). This methodology was borrowed from a research developed by the author and adviser to study pedestrian crash frequency in uncontrolled marked crosswalks (Figliozzi, Unnikrishnan, Kothuri, Caviedes, \& Soto, 2017).

To control for exposure, we estimated a VMT ratio (controlled for VMT). For segments, exposure was estimated as the segment's VMT. For intersections, exposure was estimated as the VMT of the segments that cross at the junction. For any ratio that is higher than 1, the findings suggested that there was a high concentration of pedestrian or bicyclist crashes under this condition.

To a better understanding of the next tables, some values are accompanied by *,**, or defined as NA: if the percentage of crashes or the percentage of the highway network is less than $2 \%$, the ratio is NA. If the percentage of crashes or the percentage of the highway network is higher than $2 \%$ and lower than $5 \%$, the ratio value is accompanied by $*$. If the percentage of crashes and the percentage of the highway network are higher than $2 \%$ and lower than $5 \%$, the ratio value is accompanied by $* *$. 
Equations used for the estimation of the ratio after controlling for exposure:

$$
\begin{gathered}
f_{i j k}=\frac{1}{\frac{10,000}{A A D T_{i j k}} x l_{i j k}} \\
\quad \forall i \in I \\
\forall j \in J \\
\forall k \in K
\end{gathered}
$$

\section{$\forall i \in I$ \\ $\forall \boldsymbol{j} \in \boldsymbol{J}$}

Factor for segment $\mathrm{k}$ in sub-category $\mathrm{j}$ and in category $\mathrm{i}$.

(equation 1)

$A A D T_{i j}$
$i \in I$
$j \in J$
$k \in K$
$l_{i j k}$
AADT of segment $\mathrm{k}$ in sub-category $\mathrm{j}$ and in category i.

Category analyzed (e.g. AADT)

Sub-category analyzed (e.g. ADDT between 0 and 1,000)

Length segment from the

Oregon Highway State Network

\section{Length (miles) of} segment $\mathrm{k}$ in subcategory $\mathrm{j}$ and in category $\mathrm{i}$.

VMT ratio $_{i j k}=\frac{\frac{\sum_{k} \boldsymbol{n}_{i j k}}{\sum_{i} \sum_{j} \sum_{k} \boldsymbol{n}_{i j k}}}{\frac{\sum_{j} \sum_{k} \boldsymbol{f}_{i j k}}{\sum_{i} \sum_{j} \sum_{k} \boldsymbol{f}_{i j k}}}$

Risk ratio for sub-category $\mathrm{j}$ and

(equation 2)

$$
n_{i j} k
$$$$
\text { category } i
$$

Number of pedestrian crashes in segment $\mathrm{k}$ for subcategory $\mathrm{j}$ and category $\mathrm{i}$.

$\forall i \in I$

$\forall \boldsymbol{j} \in \boldsymbol{J}$

$\forall \boldsymbol{k} \in \boldsymbol{K}$ 


\subsection{Posted speed limit}

In terms of pedestrian crashes, TABLE 31 reveals the ratio by posted speed limit. After controlling by VMT, the findings suggested that there was a high concentration of pedestrian and bicyclist crashes in roads with a posted speed limit between 20 and 35 mph (ratio higher than 1). The risk ratio tended to decrease as speed limit increased, which can be due to the few number of observations on roads with those characteristics.

Furthermore, the analysis suggested that the highest ratios were severity $\mathrm{B}, \mathrm{C}$, and $\mathrm{O}$.

TABLE 31: Pedestrian crash frequency and posted speed limit exposure ratio

\begin{tabular}{c|c|c|c|c|c}
\hline \hline $\begin{array}{c}\text { POSTED SPEED } \\
\text { LIMIT (mph) }\end{array}$ & K & A & B & C & O \\
\hline$<20$ & NA & NA & NA & NA & NA \\
\hline $20-35$ & $11.06^{*}$ & $11.75^{*}$ & $13.26^{*}$ & $13.02^{*}$ & $11.28^{*}$ \\
\hline $35-50$ & 3.59 & 4.15 & 3.66 & 3.87 & 3.78 \\
\hline $50-65$ & 0.34 & 0.21 & 0.20 & 0.18 & 0.24 \\
\hline$>65$ & NA & NA & NA & NA & NA \\
\hline \hline
\end{tabular}

TABLE 32: Bicyclist crash frequency and posted speed limit exposure ratio

\begin{tabular}{c|c|c|c|c|c}
\hline \hline $\begin{array}{c}\text { POSTED SPEED LIMIT } \\
(\mathbf{m p h})\end{array}$ & $\mathbf{K}$ & $\mathbf{A}$ & $\mathbf{B}$ & $\mathbf{C}$ & $\mathbf{O}$ \\
\hline$<20$ & $\mathrm{NA}$ & $\mathrm{NA}$ & $\mathrm{NA}$ & $\mathrm{NA}$ & $\mathrm{NA}$ \\
\hline $20-35$ & $8.95^{*}$ & $8.24^{*}$ & $11.39 *$ & $10.92^{*}$ & $7.49 *$ \\
\hline $35-50$ & 5.50 & 6.64 & 6.26 & 6.16 & 7.58 \\
\hline $50-65$ & 0.51 & 0.35 & 0.29 & 0.33 & 0.27 \\
\hline$>65$ & $\mathrm{NA}$ & $\mathrm{NA}$ & $\mathrm{NA}$ & $\mathrm{NA}$ & $\mathrm{NA}$ \\
\hline \hline
\end{tabular}

Furthermore, the findings suggested that even if roads with a posted speed limit between 50 to 65 mph represented $62 \%$ of the Oregon Highway System, most of the pedestrian and bicyclist crashes occurred on roads with a posted speed limit between 20 to $35 \mathrm{mph}$. 
TABLE 33: Proportion of road segment by posted speed limit

\begin{tabular}{c|c}
\hline \hline POSTED SPEED LIMIT & \% TOTAL \\
\hline$<20$ & $0.0 \%$ \\
\hline $20-35$ & $2.6 \%$ \\
\hline $35-50$ & $9.3 \%$ \\
\hline $50-65$ & $61.8 \%$ \\
\hline$>65$ & $26.4 \%$ \\
\hline TOTAL $(\mathrm{mi})$ & 8116.11 \\
\hline \hline
\end{tabular}

\subsection{Number of Lanes}

The findings suggested that the concentration of crashes tended to increase as the number of road lanes increased. Furthermore, most of the crashes observed occurred on 4-lane roads, and the majority tended to be severity level K and A. For bicyclist crashes, there was not a trend for the most serious crashes. TABLE 34 reveals that most of the fatal crashes occurred on roads with two lanes. For the rest of the severity levels, crashes were concentrated in 4-lane roads. Furthermore, there was a drop in the risk ratio for roads with three lanes. These roads normally have two traffic lanes and one turning lane, sometimes with a median included, which provides a refugee island for pedestrians and bicyclists, resulting in less crashes.

TABLE 34: Pedestrian crash frequency and number of lanes exposure ratio

\begin{tabular}{c|c|c|c|c|c}
\hline \hline No LANES & $\mathbf{K}$ & $\mathbf{A}$ & $\mathbf{B}$ & $\mathbf{C}$ & $\mathbf{O}$ \\
\hline 1 & NA & NA & $0.29 *$ & $0.43^{*}$ & NA \\
\hline 2 & 0.69 & 0.62 & 0.66 & 0.60 & 0.69 \\
\hline 3 & 0.37 & 0.27 & 0.41 & 0.56 & 0.86 \\
\hline 4 & 3.54 & 3.96 & 3.39 & 3.24 & 1.71 \\
\hline 5 & NA & NA & NA & NA & NA \\
\hline 6 & NA & NA & NA & NA & NA \\
\hline \hline
\end{tabular}


TABLE 35: Bicyclist crash frequency and number of lanes exposure ratio

\begin{tabular}{c|c|c|c|c|c}
\hline \hline No LANES & $\mathbf{K}$ & $\mathbf{A}$ & $\mathbf{B}$ & $\mathbf{C}$ & $\mathbf{O}$ \\
\hline 1 & NA & NA & 0.13 & $0.11^{*}$ & 0.17 \\
\hline 2 & 2.79 & 2.14 & 1.82 & 1.70 & 1.31 \\
\hline 3 & 1.09 & 0.35 & 0.59 & 0.41 & 0.56 \\
\hline 4 & 1.96 & 3.15 & 3.03 & 3.31 & 3.72 \\
\hline 5 & NA & NA & $0.47^{*}$ & $0.50^{*}$ & $0.32^{*}$ \\
\hline 6 & NA & $0.99^{* *}$ & NA & $0.63^{* *}$ & NA \\
\hline \hline
\end{tabular}

In is interesting to notice that there was a concentration of pedestrian and bicyclist crashes in 4 lane roads; however, they only make up $6 \%$ of the highway system. On the other hand, 2 lane roads represent $81 \%$ of the network, but pedestrian risk ratios are under 1. Bicyclist risk ratios are higher than 1 at these locations, but are lower that ratios for 4 lane roads.

TABLE 36: Proportion of road segment by number of lanes

\begin{tabular}{c|c}
\hline \hline NUMBER OF LANES & \% TOTAL \\
\hline 1 & $6.5 \%$ \\
\hline 2 & $80.9 \%$ \\
\hline 3 & $6.6 \%$ \\
\hline 4 & $5.9 \%$ \\
\hline 5 & $0.1 \%$ \\
\hline 6 & $0.0 \%$ \\
\hline \hline TOTAL $(\mathrm{mi})$ & 8930.46 \\
\hline
\end{tabular}

\subsection{Width}

Similar to the number of lanes category, we studied road width to explore a trend in the risk ratio by the size of the road. It was found that risk ratios tended to increase as road width increased. TABLE 37 suggests that 40-50 width roads had the highest ratio for pedestrian and bicyclist crashes. In terms of only bicyclist risk ratios, it is also important 
to notice the high concentration of crashes in roads between 20 to $30 \mathrm{ft}$. While

pedestrians were safer in narrow roads, bicyclists were found to be at risk of fatal and incapacitated injury crashes. Finally, 30-40 ft. roads were found to have a lower risk ratio, which is explained by the fact that this size is commonly found in 3 lane roads.

TABLE 37: Pedestrian crash frequency and width exposure ratio

\begin{tabular}{c|c|c|c|c|c}
\hline \hline WIDTH (ft.) & $\mathbf{K}$ & $\mathbf{A}$ & $\mathbf{B}$ & $\mathbf{C}$ & $\mathbf{O}$ \\
\hline $0-10$ & NA & NA & NA & NA & NA \\
\hline $10-20$ & NA & NA & $0.28^{*}$ & $0.36^{*}$ & NA \\
\hline $20-30$ & 0.65 & 0.52 & 0.55 & 0.51 & 0.58 \\
\hline $30-40$ & 0.44 & 0.42 & 0.56 & 0.69 & 0.83 \\
\hline $40-50$ & 3.12 & 3.49 & 3.10 & 3.01 & 2.30 \\
\hline $50-60$ & NA & NA & NA & NA & NA \\
\hline $60-70$ & NA & NA & NA & NA & NA \\
\hline$>80$ & NA & NA & NA & NA & NA \\
\hline \hline
\end{tabular}

TABLE 38: Bicyclist crash frequency and width exposure ratio

\begin{tabular}{c|c|c|c|c|c}
\hline \hline WIDTH (ft.) & $\mathbf{K}$ & $\mathbf{A}$ & $\mathbf{B}$ & $\mathbf{C}$ & $\mathbf{O}$ \\
\hline $0-10$ & NA & NA & NA & NA & NA \\
\hline $10-20$ & NA & $0.13^{*}$ & 0.29 & $0.27 *$ & 0.38 \\
\hline $20-30$ & 3.78 & 2.30 & 1.84 & 1.75 & 1.63 \\
\hline $30-40$ & 0.90 & 0.69 & 1.00 & 0.78 & 0.61 \\
\hline $40-50$ & 4.74 & 6.41 & 6.31 & 6.82 & 7.27 \\
\hline $50-60$ & NA & $2.11^{*}$ & $2.71 *$ & $2.94 *$ & $3.52 *$ \\
\hline $60-70$ & NA & $0.85^{* *}$ & $0.86 * *$ & $0.74 * *$ & $0.57 * *$ \\
\hline$>80$ & NA & $0.93^{* *}$ & NA & NA & NA \\
\hline \hline
\end{tabular}

In terms of the Oregon highway network, we found that $80 \%$ of the road has a 20 to $30 \mathrm{ft}$. width. This explained why there was a substantial concentration of bicyclist crashes at these roads. On the other hand, most pf the pedestrian crashes occurred on $40-50 \mathrm{ft}$., roads, which only represent $6 \%$ of the network. 
TABLE 39: Proportion of road segment by road width

\begin{tabular}{c|c}
\hline \hline ROAD WIDTH (ft.) & \% TOTAL \\
\hline $0-10$ & $0.0 \%$ \\
\hline $10-20$ & $6.6 \%$ \\
\hline $20-30$ & $79.7 \%$ \\
\hline $30-40$ & $7.3 \%$ \\
\hline $40-50$ & $5.6 \%$ \\
\hline $50-60$ & $0.6 \%$ \\
\hline $60-70$ & $0.2 \%$ \\
\hline$>80$ & $0.0 \%$ \\
\hline \hline TOTAL (mi) & 8930.50 \\
\hline
\end{tabular}

\subsection{Road classification}

Finally, the road classification was assessed to uncover crash patterns in Oregon. While for most of the road classification categories the data was unreliable (after controlling for VMT), results showed that most of the pedestrian and bicyclist crashes occurred in principal and minor arterials. For pedestrian crashes, the trend revealed that risk ratios were higher for fatal and severe crashes (level A) in principal arterials than any other category. For bicyclist crashes, the risk ratio was similar across the different severity levels; nonetheless, there is an increase of fatal and severe risk ratios in minor arterials.

TABLE 40: Pedestrian crash frequency and road classification exposure ratio

\begin{tabular}{c|c|c|c|c|c}
\hline \hline ROAD CLASSIFICATION & K & $\mathbf{A}$ & $\mathbf{B}$ & $\mathbf{C}$ & $\mathbf{O}$ \\
\hline Interstate & 0.18 & 0.09 & 0.11 & 0.10 & 0.31 \\
\hline Principal Arterial & 2.60 & 2.60 & 2.53 & 2.61 & 1.90 \\
\hline Minor Arterial & 1.42 & 1.71 & 1.68 & 1.48 & 0.85 \\
\hline Major collector & NA & NA & NA & NA & NA \\
\hline Minor collector & NA & NA & NA & NA & NA \\
\hline Local & NA & NA & NA & NA & NA \\
\hline \hline
\end{tabular}


TABLE 41: Bicyclist crash frequency and road classification exposure ratio

\begin{tabular}{c|c|c|c|c|c}
\hline \hline ROAD CLASSIFICATION & K & A & B & C & O \\
\hline Interstate & $0.11 *$ & $0.09 *$ & $0.08^{*}$ & $0.07 *$ & 0.17 \\
\hline Principal Arterial & 3.96 & 3.85 & 3.89 & 3.93 & 4.16 \\
\hline Minor Arterial & 1.09 & 1.05 & 0.97 & 0.82 & 0.70 \\
\hline Major collector & $0.28 *$ & $0.22 *$ & $0.13 *$ & $0.14 *$ & NA \\
\hline Minor collector & NA & NA & NA & NA & NA \\
\hline Local & NA & NA & NA & NA & NA \\
\hline \hline
\end{tabular}

In terms of the highway network, we observed that most of the crashes occurred on principal arterials since they represent $40 \%$ of the network. Interstate and freeways also have a high percentage of segments in the network; however, the concentration of crashes is lower since pedestrians and bicyclists are not allowed to travel there.

TABLE 42: Proportion of road segment by road classification

\begin{tabular}{c|c}
\hline \hline ROAD CLASSIFICATION & \% TOTAL \\
\hline Interstate/Freeway & $19.6 \%$ \\
\hline Principal Arterial & $38.9 \%$ \\
\hline Minor Arterial & $23.0 \%$ \\
\hline Rural collector & $15.9 \%$ \\
\hline Urban collector & $0.8 \%$ \\
\hline Local & $1.8 \%$ \\
\hline TOTAL (mi) & 8926.82 \\
\hline
\end{tabular}

\subsection{Neighborhood Concepts}

This section discusses the concentration of crashes by neighborhood concepts. Most of the pedestrian and bicyclist crashes that occurred on the Oregon State Highway were located in areas D, E and F. This analysis was performed to control for pedestrian and bicyclist activity (exposure), since the neighborhood concepts account for population density and employment entropy. Additionally, we considered crashes at intersections 
versus segments to have a better understanding of crash severity. Overall, the findings suggested that most of the fatal and severe crashes occurred on concepts E and F, which are mainly suburban and rural areas. After controlling by VMT, these areas revealed high risk ratios for levels $\mathrm{K}$ and $\mathrm{A}$, which can be explained by the high speed vehicles and lack of control devices. Furthermore, similar to the findings in section 3 and 4, the findings suggested that for pedestrians, most of the crashes were concentrated in segments, while for bicyclists, crashes were mainly at intersections. Table 43 shows a summary of the main findings. For more detail on risk ratio, please refer to the referenced tables.

TABLE 43: Crash frequency and neighborhood concept exposure ratio summary

\begin{tabular}{|c|c|c|c|c|c|}
\hline VAR. & $\begin{array}{c}\text { TYPE OF } \\
\text { CRASH }\end{array}$ & $\begin{array}{c}\text { NEIGH. CON. } \\
\text { D } \\
\end{array}$ & $\begin{array}{l}\text { NEIGH. } \\
\text { CON. E }\end{array}$ & $\begin{array}{c}\text { NEIGH. CON. } \\
\text { F } \\
\end{array}$ & $\begin{array}{c}\text { REF. } \\
\text { TABLE }\end{array}$ \\
\hline PSL & PED. & $\begin{array}{l}\text { Crashes were } \\
\text { concentrated in } \\
35-50 \mathrm{mph} \\
\text { speed limit } \\
\text { roads. } \\
\text { At intersections, } \\
\text { highest risk } \\
\text { ratios occurred } \\
\text { in } 20-35 \text { mph } \\
\text { roads. At } \\
\text { segments, most } \\
\text { dangerous roads } \\
\text { had a } 35-50 \mathrm{mph} \\
\text { speed limit. }\end{array}$ & $\begin{array}{l}\text { Crashes were } \\
\text { concentrated } \\
\text { in } 20-35 \text { mph } \\
\text { speed limit } \\
\text { roads. } \\
\text { Intersections } \\
\text { with } 35-50 \\
\text { mph posted } \\
\text { speed limits } \\
\text { had the } \\
\text { highest risk } \\
\text { ratios. }\end{array}$ & $\begin{array}{l}\text { The majority of } \\
\text { the observations } \\
\text { occurred on } 35- \\
50 \text { mph roads. } \\
\text { While at } \\
\text { intersections } \\
\text { most of the } \\
\text { crashes were } \\
\text { PDO, in } \\
\text { segments were } \\
\text { fatal or severe. }\end{array}$ & $\begin{array}{c}\text { TABLE } \\
61 \\
\\
\text { TABLE } \\
62 \\
\text { TABLE } \\
63\end{array}$ \\
\hline & BICYCLIST & $\begin{array}{l}\text { Most of the } \\
\text { crashes occurred } \\
\text { on roads with a } \\
\text { speed limit } \\
\text { between } 20 \text { to } 50 \\
\text { mph. } \\
\text { Risk ratios were } \\
\text { found to be } \\
\text { higher for } \\
\text { segments than } \\
\text { for intersections. }\end{array}$ & $\begin{array}{l}\text { Crashes were } \\
\text { concentrated } \\
\text { on } 20 \text { to } 35 \\
\text { mph limit } \\
\text { roads. } \\
\\
\text { Crashes } \\
\text { tended to be } \\
\text { fatal and } \\
\text { severe for } \\
\text { segments and } \\
\text { intersections. }\end{array}$ & $\begin{array}{l}\text { Crashes were } \\
\text { concentrated on } \\
20 \text { to } 35 \mathrm{mph} \\
\text { limit roads. } \\
\\
\text { While at } \\
\text { intersections } \\
\text { most of the } \\
\text { crashes were } \\
\text { PDO, in } \\
\text { segments were } \\
\text { fatal or severe. }\end{array}$ & $\begin{array}{c}\text { TABLE } \\
73 \\
\text { TABLE } \\
74 \\
\text { TABLE } \\
75\end{array}$ \\
\hline
\end{tabular}


TABLE 43: Crash frequency and neighborhood concept exposure ratio summary, continued

\begin{tabular}{|c|c|c|c|c|c|}
\hline VAR. & $\begin{array}{c}\text { TYPE } \\
\text { OF } \\
\text { CRASH }\end{array}$ & $\begin{array}{c}\text { NEIGH. CON. } \\
\text { D }\end{array}$ & $\begin{array}{l}\text { NEIGH. } \\
\text { CON. E }\end{array}$ & $\begin{array}{c}\text { NEIGH. CON. } \\
\text { F }\end{array}$ & $\begin{array}{l}\text { REF. } \\
\text { TABLE }\end{array}$ \\
\hline \multirow[t]{2}{*}{ WIDTH } & PED. & $\begin{array}{c}\text { Risk ratio } \\
\text { increased as road } \\
\text { width increased. } \\
\text { Most of the } \\
\text { crashes were } \\
\text { concentrated on } \\
50-60 \text { road } \\
\text { segments, and } \\
\text { most of them } \\
\text { were fatal or } \\
\text { severe. }\end{array}$ & $\begin{array}{l}\text { Risk ratio } \\
\text { increased as } \\
\text { road width } \\
\text { increased. } \\
\text { Risk ratios } \\
\text { remained } \\
\text { constant } \\
\text { between } \\
\text { segments and } \\
\text { intersections. }\end{array}$ & $\begin{array}{l}\text { Risk ratio } \\
\text { increased as } \\
\text { road width } \\
\text { increased. } \\
\text { 45-50 ft. roads } \\
\text { had the highest } \\
\text { risk ratios. At } \\
\text { intersections, } \\
\text { the highest risk } \\
\text { ratio was for } \\
\text { level O, while } \\
\text { for segments it } \\
\text { was level K. }\end{array}$ & $\begin{array}{c}\text { TABLE } \\
64 \\
\text { TABLE } \\
65 \\
\text { TABLE } \\
66\end{array}$ \\
\hline & BIC. & $\begin{array}{c}\text { Risk ratio } \\
\text { increased as road } \\
\text { width increased. } \\
\text { At intersections, } \\
\text { most of the } \\
\text { severe crashes } \\
\text { were } \\
\text { concentrated in } \\
50-60 \text { ft. roads. } \\
\text { For the segment } \\
\text { case, high-risk } \\
\text { ratios occurred } \\
\text { in } 40 \text { to } 50 \mathrm{ft} . \\
\text { roads. }\end{array}$ & $\begin{array}{l}\text { Risk ratio } \\
\text { increased as } \\
\text { road width } \\
\text { increased. } \\
\text { Similar trends } \\
\text { for } \\
\text { intersections } \\
\text { and segments. } \\
\text { Most of the } \\
\text { crashes } \\
\text { occurred on } \\
40 \text { to } 50 \mathrm{ft} . \\
\text { roads. }\end{array}$ & $\begin{array}{l}\text { Risk ratio } \\
\text { increased as } \\
\text { road width } \\
\text { increased. } \\
\text { Most of the } \\
\text { crashes } \\
\text { occurred on } 40 \\
\text { to } 50 \mathrm{ft} \text {. roads. } \\
\text { Intersections } \\
\text { had more PDO } \\
\text { crashes than } \\
\text { segments. }\end{array}$ & $\begin{array}{c}\text { TABLE } \\
76 \\
\text { TABLE } \\
77 \\
\text { TABLE } \\
78\end{array}$ \\
\hline $\begin{array}{c}\text { NUMBE } \\
\text { R OF } \\
\text { LANES }\end{array}$ & PED. & $\begin{array}{c}\text { Risk ratio } \\
\text { increased as } \\
\text { number of lanes } \\
\text { increased. } \\
\text { Highest risk } \\
\text { ratios occurred } \\
\text { in } 4 \text { lane roads. } \\
\text { Furthermore, } \\
\text { segments were } \\
\text { more dangerous } \\
\text { than } \\
\text { intersections. }\end{array}$ & $\begin{array}{l}\text { Risk ratio } \\
\text { increased as } \\
\text { number of } \\
\text { lanes } \\
\text { increased. } \\
\text { Most of the } \\
\text { crashes were } \\
\text { concentrated } \\
\text { at } \\
\text { intersections } \\
\text { with } 4 \text { lanes. }\end{array}$ & $\begin{array}{c}\text { Risk ratio } \\
\text { increased as } \\
\text { number of lanes } \\
\text { increased. } \\
\text { Similar to the } \\
\text { other concepts, } \\
\text { highest risk } \\
\text { ratios were on } 4 \\
\text { lane roads. At } \\
\text { intersections, } \\
\text { the highest risk } \\
\text { ratio was for } \\
\text { level O, while } \\
\text { for segments it } \\
\text { was level K. }\end{array}$ & $\begin{array}{c}\text { TABLE } \\
67 \\
\text { TABLE } \\
68 \\
\text { TABLE } \\
69\end{array}$ \\
\hline
\end{tabular}


TABLE 43: Crash frequency and neighborhood concept exposure ratio summary, continued

\begin{tabular}{|c|c|c|c|c|c|}
\hline VAR. & $\begin{array}{c}\text { TYPE } \\
\text { OF } \\
\text { CRASH }\end{array}$ & $\begin{array}{c}\text { NEIGH. CON. } \\
\text { D }\end{array}$ & $\begin{array}{l}\text { NEIGH. } \\
\text { CON. E }\end{array}$ & $\begin{array}{c}\text { NEIGH. CON. } \\
\text { F }\end{array}$ & $\begin{array}{l}\text { REF. } \\
\text { TABLE }\end{array}$ \\
\hline $\begin{array}{c}\text { NUMBER } \\
\text { OF } \\
\text { LANES }\end{array}$ & BIC. & $\begin{array}{c}\text { Risk ratio } \\
\text { increased as } \\
\text { number of } \\
\text { lanes } \\
\text { increased. } \\
\text { Four lane roads } \\
\text { had the highest } \\
\text { risk ratios. }\end{array}$ & $\begin{array}{l}\text { Risk ratio } \\
\text { increased as } \\
\text { number of } \\
\text { lanes } \\
\text { increased. } \\
\text { Four lane } \\
\text { roads had the } \\
\text { highest risk } \\
\text { ratios. There } \\
\text { were more } \\
\text { fatal crashes } \\
\text { at } \\
\text { intersections } \\
\text { than in } \\
\text { segments. }\end{array}$ & $\begin{array}{c}\text { Risk ratio } \\
\text { increased as } \\
\text { number of } \\
\text { lanes increased. } \\
\text { Most of the } \\
\text { crashes } \\
\text { occurred on } \\
\text { four lane roads, } \\
\text { specifically at } \\
\text { intersections. }\end{array}$ & $\begin{array}{c}\text { TABLE } \\
79 \\
\text { TABLE } \\
80 \\
\\
\text { TABLE } \\
81\end{array}$ \\
\hline $\begin{array}{l}\text { ROAD } \\
\text { CLASS. }\end{array}$ & PED. & $\begin{array}{l}\text { Most of the } \\
\text { crashes } \\
\text { occurred on } \\
\text { principal and } \\
\text { minor arterials. } \\
\text { The trend of } \\
\text { risk ratios was } \\
\text { similar } \\
\text { between } \\
\text { intersections } \\
\text { and segments. }\end{array}$ & $\begin{array}{l}\text { Most of the } \\
\text { crashes } \\
\text { occurred on } \\
\text { principal and } \\
\text { minor } \\
\text { arterials. } \\
\text { The trend of } \\
\text { risk ratios was } \\
\text { similar } \\
\text { between } \\
\text { intersections } \\
\text { and segments. }\end{array}$ & $\begin{array}{l}\text { Most of the } \\
\text { crashes } \\
\text { occurred on } \\
\text { principal and } \\
\text { minor arterials. } \\
\text { Segments had } \\
\text { higher risk } \\
\text { rations than } \\
\text { intersections. }\end{array}$ & $\begin{array}{c}\text { TABLE } \\
70 \\
\text { TABLE } \\
71 \\
\\
\text { TABLE } \\
72\end{array}$ \\
\hline & BIC. & $\begin{array}{l}\text { Most of the } \\
\text { crashes } \\
\text { occurred on } \\
\text { principal and } \\
\text { minor arterials. } \\
\text { Intersections } \\
\text { had higher } \\
\text { concentrations } \\
\text { of crashes than } \\
\text { segments. }\end{array}$ & $\begin{array}{l}\text { Most of the } \\
\text { crashes } \\
\text { occurred on } \\
\text { principal and } \\
\text { minor } \\
\text { arterials. } \\
\text { Most of the } \\
\text { fatal crashes } \\
\text { occurred on } \\
\text { segments. }\end{array}$ & $\begin{array}{l}\text { Most of the } \\
\text { crashes } \\
\text { occurred on } \\
\text { principal and } \\
\text { minor arterials. } \\
\text { Intersections } \\
\text { had higher } \\
\text { concentrations } \\
\text { of crashes than } \\
\text { segments. }\end{array}$ & $\begin{array}{c}\text { TABLE } \\
82 \\
\text { TABLE } \\
83 \\
\text { TABLE } \\
84\end{array}$ \\
\hline
\end{tabular}

Risk ratios were higher in rural areas (concepts $\mathrm{E}$ and $\mathrm{F}$ ), which can be explained by the high percentage of segments that go through these land uses. 
TABLE 44: Proportion of road segment by neighborhood concepts

\begin{tabular}{c|c}
\hline \hline NEIGH. CONCEPT & \% TOTAL \\
\hline $\mathrm{D}$ & $3.0 \%$ \\
\hline $\mathrm{E}$ & $60.4 \%$ \\
\hline $\mathrm{F}$ & $36.6 \%$ \\
\hline TOTAL (mi) & 8116.11 \\
\hline \hline
\end{tabular}

\subsection{Summary}

This section presented the findings of a crash analysis after trying to account for exposure based on VMT and land use (neighborhood concepts). These results reinforced some of the findings of the literature review and provided a preliminary indication of the potential variables that increased severity levels. Furthermore, in terms of the research questions, we found that exposure allowed us to identify how road characteristics affect crash severity, even after controlling for traffic exposure (VMT) and vulnerable user activity (land use=neighborhood concepts). 
TABLE 45: Exposure analysis summary

\begin{tabular}{|c|c|c|c|}
\hline \multirow[t]{2}{*}{ TYPE } & \multirow[t]{2}{*}{ VARIABLE } & \multicolumn{2}{|c|}{ FINDINGS } \\
\hline & & $\begin{array}{c}\text { PEDESTRIAN } \\
\text { CRASHES }\end{array}$ & $\begin{array}{c}\text { BICYCLIST } \\
\text { CRASHES } \\
\end{array}$ \\
\hline \multirow[t]{4}{*}{$\begin{array}{c}\text { ROAD } \\
\text { CHARACTERISTICS }\end{array}$} & $\begin{array}{l}\text { POSTED SPEED } \\
\text { LIMIT }\end{array}$ & $\begin{array}{l}\text { The highest risk } \\
\text { ratio was for roads } \\
\text { with a posted speed } \\
\text { limit between } 20 \text { to } \\
\quad 35 \mathrm{mph} \text {. }\end{array}$ & $\begin{array}{c}\text { The highest risk } \\
\text { ratio was for roads } \\
\text { with a posted } \\
\text { speed limit } \\
\text { between } 20 \text { to } 35 \\
\text { mph. } \\
\end{array}$ \\
\hline & $\begin{array}{l}\text { NUMBER OF } \\
\text { LANES }\end{array}$ & $\begin{array}{l}\text { Fatal crashes were } \\
\text { concentrated in } 4 \\
\text { lane roads. Severity } \\
\text { increased as } \\
\text { number of lanes } \\
\text { increased. }\end{array}$ & $\begin{array}{c}\text { Fatal and severe } \\
\text { crashes were } \\
\text { concentrated in } 2 \\
\text { lane roads. } \\
\text { Severity increased } \\
\text { as number of lanes } \\
\text { increased. }\end{array}$ \\
\hline & WIDTH & $\begin{array}{l}\text { Road widths } \\
\text { between } 40 \text { and } 50 \\
\text { ft. had the highest } \\
\text { risk ratio. } \\
\text { Severity increased } \\
\text { as road width } \\
\text { increased. }\end{array}$ & $\begin{array}{c}\text { Road widths } \\
\text { between } 40 \text { and } 50 \\
\text { ft. had the highest } \\
\text { risk ratio. } \\
\text { Severity increased } \\
\text { as road width } \\
\text { increased; } \\
\text { however, most of } \\
\text { the crashes were } \\
\text { level O. }\end{array}$ \\
\hline & $\begin{array}{c}\text { ROAD } \\
\text { CLASSIFICATION }\end{array}$ & $\begin{array}{l}\text { Most of the crashes } \\
\text { were concentrated } \\
\text { in arterials. }\end{array}$ & $\begin{array}{l}\text { Most of the } \\
\text { crashes were } \\
\text { concentrated in } \\
\text { arterials. Risk } \\
\text { ratios for fatal and } \\
\text { severe crashes } \\
\text { were higher than } \\
\text { for pedestrian } \\
\text { crashes. }\end{array}$ \\
\hline LAND USE & $\begin{array}{l}\text { NEIGHBORHOOD } \\
\text { CONCEPTS }\end{array}$ & $\begin{array}{l}\text { Most of the crashes } \\
\text { were concentrated } \\
\text { in concepts E and } \\
\text { F. } \\
\text { There were more } \\
\text { fatal and severe } \\
\text { crashes for } \\
\text { segments than for } \\
\text { intersections. }\end{array}$ & $\begin{array}{c}\text { Similar to } \\
\text { pedestrians, } \\
\text { crashes were } \\
\text { concentrated in } \\
\text { suburban and rural } \\
\text { areas. } \\
\text { More crashes } \\
\text { occurred at } \\
\text { intersections. }\end{array}$ \\
\hline
\end{tabular}




\section{DATA ANALYSIS AND RESULTS}

After identifying potential risk factors and trends in the descriptive and exposure analysis, the next chapters are going to be focused on statistical methodologies to predict pedestrian and bicyclist crash severity in terms of risk factors. The database contained a total of 13,309 crashes, where 6,162 involved pedestrians and 7,147 involved people riding a bicycle. Since this study was targeted to ODOT's facilities, only crashes that fell in the ODOT highway network were analyzed in this section (1,649 pedestrian crashes and 1,214 bicyclist crashes). Furthermore, we dropped observations that were not useful in this study (i.e. crashes at interstates), resulting in 1,535 pedestrian crashes and 1,000 bicyclist observations.

Most of the studies reviewed have explored crashes using methodologies that took into consideration the categorical and ordinal nature of severity. Based on these examples

and the crash severity modelling review by Savolainen et al. (2011), an ordinal regression methodology was chosen. The ordinal regression is a statistical tool to predict an ordinal dependent variable (e.g. severity level) in terms of other independent variables. The parameters estimated in the model represent the $\log$ of the odds that an event occurs. For example, the odds that a fatal crash occurs is the ratio of the probability of a fatal crash to the probability of a non-fatal crash. The ordinal logistic regression model results presented in this section were estimated with the use of the R package ordinal (Christensen, n.d.) and MASS (Ripley, et al., n.d.).

The variables explored in the models are summarized in TABLE 46: 11\% of pedestrian crashes were reported as fatal, while $16 \%$ incapacitated injury. For bicyclist crashes, only $2 \%$ were fatal, while $7 \%$ were reported as incapacitated injury. While some 
risk factors such as age and posted speed limit (for pedestrian crashes) showed an increase in severity as the variable increased, most of the factors did not show a pattern. It should be noted that for the estimation of the models, some non-significant levels were used as part of the base group. 
TABLE 46: Distribution (\%) of risk factors for different severity levels

\begin{tabular}{|c|c|c|c|c|c|c|c|c|c|c|c|c|c|}
\hline \multirow[b]{2}{*}{ CATEGORY } & \multirow[b]{2}{*}{ VARIABLE } & \multirow{2}{*}{$\begin{array}{c}\text { n PED. } \\
\text { CRASHES }\end{array}$} & \multirow{2}{*}{$\begin{array}{c}\text { n BIC. } \\
\text { CRASHES }\end{array}$} & \multicolumn{5}{|c|}{ PED. CRASHES (\%) } & \multicolumn{5}{|c|}{$\begin{array}{c}\text { BIC. CRASHES } \\
(\%)\end{array}$} \\
\hline & & & & $\mathbf{K}$ & $\mathbf{A}$ & B & C & $\mathbf{O}$ & $\mathbf{K}$ & $\mathbf{A}$ & B & C & $\mathbf{O}$ \\
\hline \multirow{7}{*}{$\begin{array}{l}\text { Location } \\
\text { charac. }\end{array}$} & Location & & & & & & & & & & & & \\
\hline & Segment & 1039 & 867 & 5.3 & 13.1 & 46.1 & 34.8 & 0.7 & 1.0 & 6.1 & 57.7 & 31.9 & 3.2 \\
\hline & Intersection & 496 & 133 & 20.0 & 21.4 & 36.3 & 21.8 & 0.6 & 7.5 & 12.8 & 57.1 & 21.1 & 1.5 \\
\hline & Land Use & & & & & & & & & & & & \\
\hline & Concept $\mathrm{C}$ & 602 & 372 & 6.0 & 14.6 & 45.8 & 33.1 & 0.5 & 0.8 & 6.7 & 60.2 & 29.6 & 2.7 \\
\hline & Concept D & 724 & 487 & 12.4 & 16.9 & 40.1 & 29.8 & 0.8 & 2.9 & 7.4 & 55.0 & 30.8 & 3.9 \\
\hline & Concept F & 209 & 141 & 13.4 & 15.3 & 44.5 & 26.3 & 0.5 & 1.4 & 6.4 & 59.6 & 31.9 & 0.7 \\
\hline \multirow{13}{*}{$\begin{array}{l}\text { Environmental } \\
\text { charac. }\end{array}$} & Season & & & & & & & & & & & & \\
\hline & Summer & 284 & 361 & 9.9 & 14.8 & 46.8 & 27.8 & 0.7 & 1.1 & 6.4 & 60.7 & 28.3 & 3.6 \\
\hline & Fall & 270 & 210 & 11.9 & 15.6 & 46.7 & 25.2 & 0.7 & 3.3 & 6.7 & 56.7 & 30.5 & 2.9 \\
\hline & Winter & 662 & 203 & 11.2 & 14.5 & 38.4 & 35.3 & 0.6 & 2.0 & 10.3 & 51.2 & 34.0 & 2.5 \\
\hline & Spring & 319 & 226 & 6.3 & 19.4 & 45.8 & 27.9 & 0.6 & 1.8 & 5.3 & 59.3 & 31.0 & 2.7 \\
\hline & Day of the week & & & & & & & & & & & & \\
\hline & Monday & 137 & 77 & 14.6 & 15.3 & 46.7 & 23.4 & 0.0 & 2.6 & 5.2 & 62.3 & 26.0 & 3.9 \\
\hline & Tuesday & 247 & 167 & 10.9 & 17.8 & 39.3 & 31.6 & 0.4 & 0.0 & 9.6 & 53.9 & 33.5 & 3.0 \\
\hline & Wednesday & 219 & 162 & 6.4 & 16.4 & 42.0 & 34.7 & 0.5 & 1.9 & 5.6 & 58.0 & 32.7 & 1.9 \\
\hline & Thursday & 235 & 164 & 9.8 & 11.9 & 45.1 & 32.3 & 0.9 & 1.8 & 4.3 & 58.5 & 28.7 & 6.7 \\
\hline & Friday & 225 & 161 & 8.4 & 17.3 & 45.8 & 28.4 & 0.0 & 1.9 & 9.3 & 59.0 & 29.2 & 0.6 \\
\hline & Saturday & 284 & 145 & 9.2 & 13.0 & 45.4 & 31.3 & 1.1 & 1.4 & 5.5 & 60.7 & 31.0 & 1.4 \\
\hline & Sunday & 188 & 124 & 13.3 & 19.7 & 36.2 & 29.3 & 1.6 & 4.8 & 8.9 & 52.4 & 29.8 & 4.0 \\
\hline
\end{tabular}




\section{TABLE 46: Distribution (\%) of risk factors for different severity levels, continued}

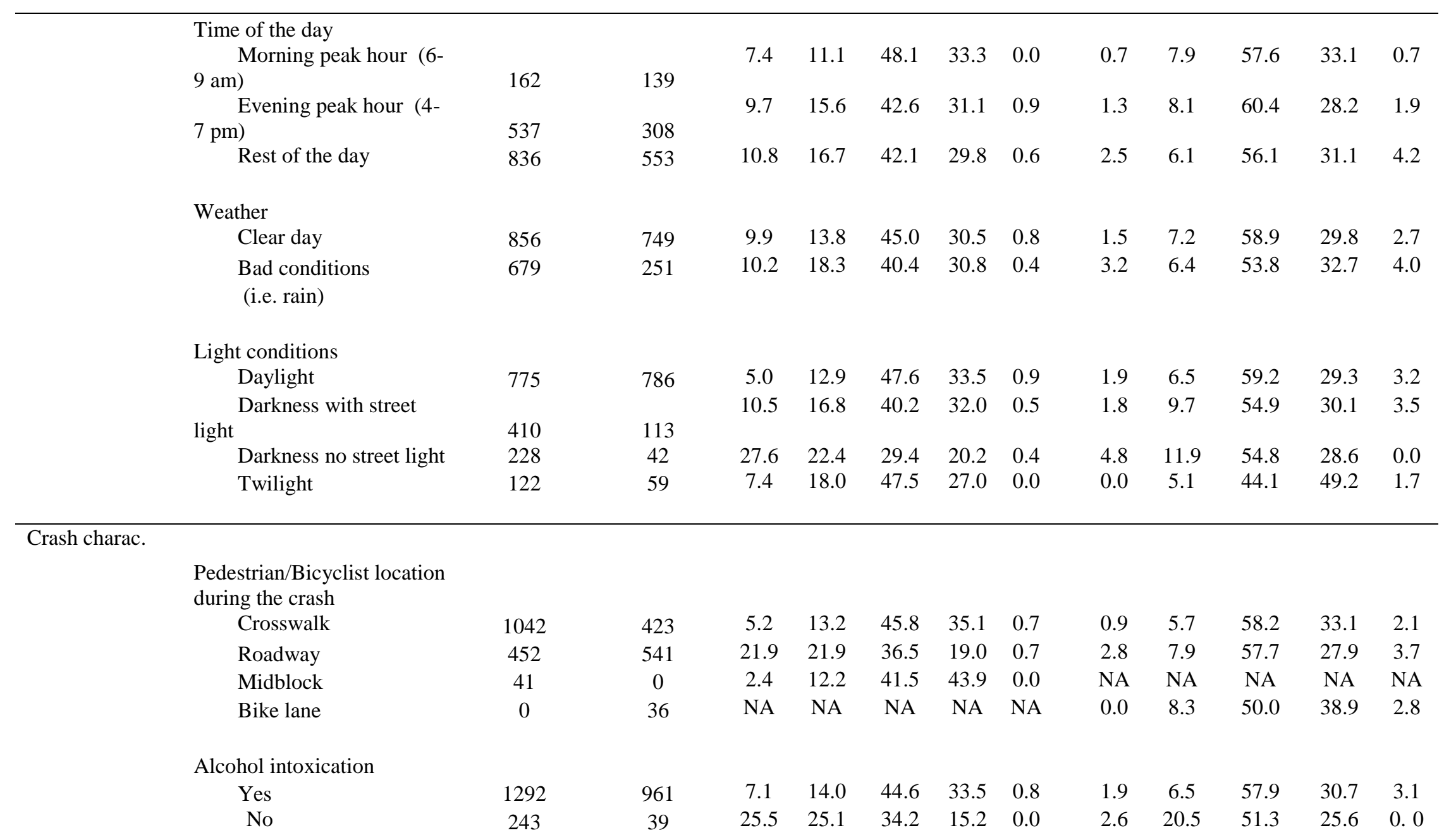


TABLE 46: Distribution (\%) of risk factors for different severity levels, continued

\begin{tabular}{|c|c|c|c|c|c|c|c|c|c|c|c|c|c|}
\hline \multicolumn{14}{|c|}{ Motor vehicle movement } \\
\hline & Straight & 893 & 393 & 14.7 & 21.4 & 40.1 & 23.1 & 0.8 & 4.6 & 11.2 & 57.0 & 23.7 & 3.6 \\
\hline & Turning & 642 & 607 & 3.6 & 7.9 & 46.9 & 41.1 & 0.5 & 0.2 & 4.3 & 58.0 & 34.9 & 2.6 \\
\hline & \multicolumn{13}{|l|}{ Vehicle type } \\
\hline & Passenger vehicle & 1496 & 989 & 9.6 & 15.8 & 43.0 & 30.9 & 0.7 & 1.6 & 7.0 & 57.9 & 30.4 & 3.0 \\
\hline & Truck and buses & 39 & 11 & 28.2 & 15.4 & 38.5 & 17.9 & 0.0 & 27.3 & 9.1 & 27.3 & 36.4 & 0.0 \\
\hline \multirow{7}{*}{$\begin{array}{l}\text { Demographic } \\
\text { charac. }\end{array}$} & \multicolumn{13}{|l|}{ Age } \\
\hline & $<=54$ & 1188 & 856 & 7.6 & 15.8 & 43.4 & 32.3 & 0.8 & 1.3 & 6.3 & 56.8 & 32.2 & 3.4 \\
\hline & $55-74$ & 273 & 127 & 14.3 & 15.4 & 42.5 & 27.8 & 0.0 & 3.9 & 8.7 & 65.4 & 21.3 & 0.8 \\
\hline & $>74$ & 74 & 17 & 33.8 & 16.2 & 36.5 & 13.5 & 0.0 & 17.6 & 29.4 & 41.2 & 11.8 & 0.0 \\
\hline & \multicolumn{13}{|l|}{ Gender } \\
\hline & Male & 909 & 754 & 12.3 & 16.6 & 40.5 & 30.1 & 0.4 & 2.0 & 6.8 & 57.4 & 30.6 & 3.2 \\
\hline & Female & 626 & 246 & 6.7 & 14.5 & 46.5 & 31.3 & 1.0 & 1.6 & 7.7 & 58.1 & 30.1 & 2.4 \\
\hline \multirow[t]{10}{*}{$\begin{array}{l}\text { Traffic } \\
\text { conditions }\end{array}$} & \multicolumn{13}{|l|}{ AADT } \\
\hline & $0-5,000$ & 88 & 70 & 9.1 & 17.0 & 46.6 & 27.3 & 0.0 & 5.7 & 11.4 & 57.1 & 25.7 & 0.0 \\
\hline & $5,000-20,000$ & 830 & 536 & 11.6 & 15.2 & 42.8 & 29.6 & 0.8 & 1.5 & 6.3 & 59.3 & 30.4 & 2.4 \\
\hline & $20,000-50,000$ & 613 & 388 & 8.0 & 16.3 & 42.6 & 32.6 & 0.5 & 1.8 & 7.2 & 55.2 & 31.4 & 4.4 \\
\hline & $>50,000$ & 4 & 6 & 25.0 & 25.0 & 50.0 & 0.0 & 0.0 & 0.0 & 0.0 & 66.7 & 33.3 & 0.0 \\
\hline & \multicolumn{13}{|l|}{ AADT (only trucks) } \\
\hline & $0-1,500$ & 1107 & 716 & 10.6 & 14.8 & 41.8 & 32.2 & 0.6 & 1.8 & 7.3 & 59.8 & 28.5 & 2.7 \\
\hline & $1,500-5,000$ & 418 & 271 & 8.9 & 18.2 & 45.7 & 26.6 & 0.7 & 2.2 & 6.3 & 51.7 & 35.8 & 4.1 \\
\hline & $5,000-7,500$ & 9 & 8 & 0.0 & 22.2 & 44.4 & 33.3 & 0.0 & 0.0 & 12.5 & 62.5 & 25.0 & 0.0 \\
\hline & $>7,500$ & 1 & 5 & 0.0 & 0.0 & 100.0 & 0.0 & 0.0 & 0.0 & 0.0 & 60.0 & 40.0 & 0.0 \\
\hline
\end{tabular}


TABLE 46: Distribution (\%) of risk factors for different severity levels, continued

\begin{tabular}{|c|c|c|c|c|c|c|c|c|c|c|c|c|c|}
\hline \multicolumn{14}{|l|}{ Road charac. } \\
\hline & Number of lanes & & & & & & & & & & & & \\
\hline & 1 & 26 & 40 & 0.0 & 3.8 & 53.8 & 42.3 & 0.0 & 0.0 & 2.5 & 60.0 & 37.5 & 0.0 \\
\hline & 2 & 513 & 341 & 10.1 & 15.0 & 44.2 & 29.6 & 1.0 & 2.9 & 8.8 & 57.8 & 28.4 & 2.1 \\
\hline & 3 & 139 & 86 & 5.8 & 11.5 & 39.6 & 41.7 & 1.4 & 2.3 & 3.5 & 67.4 & 20.9 & 5.8 \\
\hline & 4 & 812 & 500 & 11.2 & 17.7 & 42.2 & 28.6 & 0.2 & 1.4 & 6.8 & 54.8 & 33.4 & 3.6 \\
\hline & 5 & 36 & 24 & 5.6 & 5.6 & 52.8 & 33.3 & 2.8 & 0.0 & 0.0 & 70.8 & 29.2 & 0.0 \\
\hline & 6 & 9 & 13 & 11.1 & 22.2 & 11.1 & 55.6 & 0.0 & 0.0 & 15.4 & 46.2 & 38.5 & 0.0 \\
\hline \multicolumn{14}{|c|}{ Width } \\
\hline \multicolumn{14}{|c|}{$0-10$} \\
\hline & $10-20$ & 24 & 38 & 0.0 & 4.2 & 58.3 & 37.5 & 0.0 & 0.0 & 2.6 & 57.9 & 39.5 & 0.0 \\
\hline & $20-30$ & 420 & 285 & 11.7 & 15.0 & 42.9 & 29.3 & 1.2 & 3.5 & 9.1 & 56.1 & 28.8 & 2.5 \\
\hline & $30-40$ & 192 & 114 & 5.2 & 12.5 & 42.2 & 39.6 & 0.5 & 0.0 & 6.1 & 68.4 & 23.7 & 1.8 \\
\hline & $40-50$ & 680 & 448 & 10.9 & 17.5 & 42.2 & 29.0 & 0.4 & 2.0 & 6.5 & 55.1 & 32.6 & 3.8 \\
\hline & $50-60$ & 175 & 84 & 10.9 & 16.6 & 45.1 & 27.4 & 0.0 & 0.0 & 6.0 & 58.3 & 31.0 & 4.8 \\
\hline & $60-70$ & 36 & 26 & 5.6 & 11.1 & 47.2 & 33.3 & 2.8 & 0.0 & 3.8 & 73.1 & 23.1 & 0.0 \\
\hline & $>70$ & 8 & 5 & 0.0 & 25.0 & 12.5 & 62.5 & 0.0 & 0.0 & 20.0 & 20.0 & 60.0 & 0.0 \\
\hline \multicolumn{14}{|c|}{ Road classification } \\
\hline & Arterials & 1438 & 913 & 10.6 & 15.9 & 42.6 & 30.5 & 0.5 & 0.0 & 0.0 & 0.0 & 0.0 & 0.0 \\
\hline & Collectors & 53 & 44 & 1.9 & 13.2 & 56.6 & 24.5 & 3.8 & 38.6 & 145.5 & 1204.5 & 625.0 & 61.4 \\
\hline & Local streets & 44 & 43 & 0.0 & 15.9 & 38.6 & 43.2 & 2.3 & 4.7 & 7.0 & 58.1 & 30.2 & 2.3 \\
\hline \multicolumn{14}{|c|}{ Road surface } \\
\hline & Dry & 1075 & 862 & 10.2 & 16.5 & 44.1 & 28.5 & 0.7 & 2.0 & 7.1 & 59.4 & 28.8 & 2.8 \\
\hline & Wet & 460 & 138 & 9.6 & 14.1 & 40.2 & 35.7 & 0.4 & 1.4 & 6.5 & 46.4 & 41.3 & 4.3 \\
\hline
\end{tabular}


TABLE 46: Distribution (\%) of risk factors for different severity levels, continued

\begin{tabular}{|c|c|c|c|c|c|c|c|c|c|c|c|c|}
\hline \multicolumn{13}{|l|}{ Traffic control device } \\
\hline Other devices & 1492 & 992 & 10.2 & 15.9 & 42.2 & 31.0 & 0.7 & 0.0 & 0.0 & 87.5 & 12.5 & 0.0 \\
\hline Pedestrian signal & 43 & 8 & 4.7 & 11.6 & 67.4 & 16.3 & 0.0 & 1.9 & 7.1 & 57.4 & 30.6 & 3.0 \\
\hline \multicolumn{13}{|l|}{ Posted speed limit } \\
\hline$<=20$ & 55 & 26 & 1.8 & 5.5 & 50.9 & 38.2 & 3.6 & 0.0 & 0.0 & 69.2 & 30.8 & 0.0 \\
\hline $20-35$ & 1032 & 616 & 6.5 & 14.6 & 44.7 & 33.7 & 0.5 & 1.0 & 5.4 & 60.4 & 29.7 & 3.6 \\
\hline $35-50$ & 316 & 239 & 19.6 & 18.4 & 38.0 & 23.4 & 0.6 & 2.9 & 10.0 & 54.0 & 30.5 & 2.5 \\
\hline $50-65$ & 131 & 114 & 18.3 & 22.1 & 38.2 & 20.6 & 0.8 & 5.3 & 10.5 & 47.4 & 35.1 & 1.8 \\
\hline$>=65$ & 1 & 5 & 0.0 & 100.0 & 0.0 & 0.0 & 0.0 & 0.0 & 20.0 & 60.0 & 20.0 & 0.0 \\
\hline
\end{tabular}

$\overline{N A=\text { Not applicable }}$ 


\subsection{Single variable models}

As a preliminary exploratory analysis, we conducted an ordinal regression model to explore KABCO levels by each independent variable. The next sections present the results in terms of odds.

\subsubsection{Location characteristics}

In terms of the location of the crash, the findings suggested that the odds of fatal crashes were higher in segments than at intersections. This is true for pedestrian and bicyclist crashes. Furthermore, TABLE 47 reveals that pedestrian and bicyclist crashes that occurred in suburban and rural areas (concept $\mathrm{E}$ and F), tended to increase the odds of a fatal outcome. For bicyclist crashes, we did not find a statistical difference in concept $F$ and $\mathrm{E}$, versus concept $\mathrm{D}$.

TABLE 47: Single variable model - location characteristics (odd ratios)

\begin{tabular}{|c|c|c|c|c|c|c|c|}
\hline & VAR. & BASE & LEVEL & MEAN & $95 \%$ & \multicolumn{2}{|c|}{ P-VALUE } \\
\hline \multirow[t]{3}{*}{ Ped. } & Location & Intersection & Segment & 2.62 & 2.15 & 3.21 & 0.00 \\
\hline & Land Use & Concept D & $\begin{array}{c}\text { Concept } \\
\text { E }\end{array}$ & 1.28 & 1.05 & 1.57 & 0.01 \\
\hline & & & $\begin{array}{c}\text { Concept } \\
\text { F }\end{array}$ & 1.46 & 1.10 & 1.93 & 0.00 \\
\hline \multirow[t]{3}{*}{ Bic. } & Location & Intersection & Segment & 2.06 & 1.47 & 2.89 & 0.00 \\
\hline & Land Use & Concept D & $\begin{array}{c}\text { Concept } \\
\text { E }\end{array}$ & 1.09 & 0.85 & 1.38 & 0.51 \\
\hline & & & $\begin{array}{c}\text { Concept } \\
\mathrm{F}\end{array}$ & 1.07 & 0.75 & 1.51 & 0.72 \\
\hline
\end{tabular}

\subsubsection{Environmental conditions}

The findings did not suggest significant differences in terms of season. In terms of day of the week and vulnerable user crashes, the findings did not suggest a statistical 
relationship with severity. On the other hand, time of the day showed some significant differences. Pedestrian crashes during morning peak hours tended to be less severe than crashes that occurred during non-peak hours. The high traffic volumes in peak hour resulted in vehicles traveling at lower speeds, which ended up reducing severity when a crash occurred.

Considering weather conditions, the results showed that cloudy days increased the severity of crashes. On the other hand, rainy days decreased it at a significant level (for both pedestrian and bicyclist crashes); however, the finding is non-significant. This last finding suggested that drivers may tend to travel at lower speeds when it is raining, which results in less severe crashes.

Finally, the results for light conditions only showed a significant association for pedestrian crashes. Crashes that occurred during dark light conditions tended to be more severe.

TABLE 48: Single variable model - environmental conditions (odd ratios)

\begin{tabular}{|c|c|c|c|c|c|c|c|}
\hline & VAR. & BASE & LEVEL & MEAN & \multicolumn{2}{|c|}{ 95\% C.I. } & P- \\
\hline \multirow[t]{9}{*}{ Ped } & Season & Summer & Fall & 1.01 & 0.76 & 1.38 & 0.93 \\
\hline & & & Winter & 0.78 & 0.61 & 1.01 & 0.06 \\
\hline & & & Spring & 0.90 & 0.67 & 1.20 & 0.48 \\
\hline & Day of & Sunday & Monday & 1.10 & 0.73 & 1.65 & 0.64 \\
\hline & week & & Tuesday & 0.83 & 0.58 & 1.18 & 0.30 \\
\hline & & & Wednesda & 0.72 & 0.51 & 1.04 & 0.08 \\
\hline & & & $\begin{array}{c}\mathrm{y} \\
\text { Thursday }\end{array}$ & 0.75 & 0.53 & 1.07 & 0.11 \\
\hline & & & Friday & 0.91 & 0.63 & 1.30 & 0.59 \\
\hline & & & Saturday & 0.76 & 0.53 & 1.06 & 0.10 \\
\hline
\end{tabular}


TABLE 48: Single variable model - environmental conditions (odd ratios), continued

\begin{tabular}{|c|c|c|c|c|c|c|c|}
\hline & $\begin{array}{l}\text { Time of } \\
\text { the day }\end{array}$ & $\begin{array}{l}\text { Rest of } \\
\text { the day }\end{array}$ & $\begin{array}{l}\text { Morning } \\
\text { peak hour } \\
\text { (6-9 a.m.) } \\
\text { Evening } \\
\text { peak hour } \\
\text { (4-7 p.m.) }\end{array}$ & 0.96 & 0.78 & 1.18 & 0.71 \\
\hline & \multirow[t]{2}{*}{$\begin{array}{l}\text { Weathe } \\
\text { r cond. }\end{array}$} & \multirow[t]{2}{*}{$\begin{array}{c}\text { Clear } \\
\text { day }\end{array}$} & Cloudy & 1.44 & 1.14 & 1.84 & 0.00 \\
\hline & & & Rain & 0.82 & 0.65 & 1.03 & 0.09 \\
\hline & \multirow[t]{4}{*}{$\begin{array}{l}\text { Light } \\
\text { cond. }\end{array}$} & \multirow[t]{4}{*}{ Daylight } & $\begin{array}{c}\text { Darkness } \\
\text { with street } \\
\text { lights }\end{array}$ & 1.33 & 1.06 & 1.65 & 0.01 \\
\hline & & & $\begin{array}{l}\text { Darkness } \\
\text { with no } \\
\text { street light }\end{array}$ & 3.05 & 2.30 & 4.05 & 0.00 \\
\hline & & & $\begin{array}{l}\text { Dawn } \\
\text { twilight }\end{array}$ & 1.33 & 0.73 & 2.42 & 0.50 \\
\hline & & & $\begin{array}{c}\text { Dusk } \\
\text { twilight }\end{array}$ & 1.63 & 1.09 & 2.45 & 0.01 \\
\hline \multirow[t]{11}{*}{ Bic. } & \multirow[t]{3}{*}{ Season } & \multirow[t]{3}{*}{ Summer } & Fall & 0.98 & 0.71 & 1.33 & 0.87 \\
\hline & & & Winter & 0.96 & 0.70 & 1.31 & 0.78 \\
\hline & & & Spring & 0.92 & 0.68 & 1.24 & 0.58 \\
\hline & \multirow{4}{*}{$\begin{array}{c}\text { Day of } \\
\text { the } \\
\text { week }\end{array}$} & \multirow[t]{4}{*}{ Sunday } & Monday & 0.93 & 0.56 & 1.59 & 0.78 \\
\hline & & & Tuesday & 0.79 & 0.52 & 1.26 & 0.31 \\
\hline & & & Wednesda & 0.77 & 0.50 & 1.49 & 0.24 \\
\hline & & & $\begin{array}{c}\text { y } \\
\text { Thursday } \\
\text { Friday } \\
\text { Saturday }\end{array}$ & $\begin{array}{l}0.82 \\
0.97 \\
0.85\end{array}$ & $\begin{array}{l}0.54 \\
0.63 \\
0.55\end{array}$ & $\begin{array}{l}1.27 \\
1.49 \\
1.32\end{array}$ & $\begin{array}{l}0.39 \\
0.89 \\
0.48\end{array}$ \\
\hline & \multirow[t]{2}{*}{$\begin{array}{l}\text { Time of } \\
\text { the day }\end{array}$} & \multirow[t]{2}{*}{$\begin{array}{l}\text { Rest of } \\
\text { the day }\end{array}$} & $\begin{array}{l}\text { Morning } \\
\text { peak hour } \\
(6-9 \text { a.m.) }\end{array}$ & 1.23 & 0.88 & 1.72 & 0.23 \\
\hline & & & $\begin{array}{l}\text { Evening } \\
\text { peak hour } \\
(4-7 \text { p.m. })\end{array}$ & 1.16 & 0.91 & 1.49 & 0.24 \\
\hline & \multirow[t]{2}{*}{$\begin{array}{l}\text { Weathe } \\
\text { r cond. }\end{array}$} & $\begin{array}{l}\text { Clear } \\
\text { day }\end{array}$ & Cloudy & 1.06 & 0.77 & 1.45 & 0.71 \\
\hline & & & Rain & 0.66 & 0.44 & 0.98 & 0.04 \\
\hline
\end{tabular}


TABLE 48: Single variable model - environmental conditions (odd ratios), continued

\begin{tabular}{cccccc}
$\begin{array}{l}\text { Light } \\
\text { cond. }\end{array}$ Daylight & $\begin{array}{c}\text { Darkness } \\
\text { with street } \\
\text { lights } \\
\text { Darkness } \\
\text { with no } \\
\text { street light } \\
\text { Dawn } \\
\text { twilight } \\
\text { Dusk } \\
\text { twilight }\end{array}$ & 0.93 & 0.65 & 1.34 & 0.70 \\
\hline
\end{tabular}

\subsubsection{Crash characteristics}

Four variables were considered for crash characteristics: location during the crash, alcohol intoxication, vehicle movement, and vehicle type.

The findings suggested that pedestrian crashes that were outside the crosswalk tended to be more severe than crashes that occurred in the crosswalk. In terms of alcohol intoxication, the results are intuitive as well. Pedestrian and bicyclist crashes caused by an impaired driver tended to increase the odds of a severe crash.

In terms of vehicles movements, crashes caused by a vehicle moving straight had higher odds to be fatal than crashes caused by vehicles turning. These results were significant for the pedestrian and bicyclist models. Furthermore, heavy vehicles (i.e. trucks and buses) tended to increase the odds of fatal pedestrian and bicyclist crashes. 
TABLE 49: Single variable model - crash characteristics (odd ratios)

\begin{tabular}{|c|c|c|c|c|c|c|c|}
\hline & VAR. & BASE & LEVEL & MEA & \multicolumn{2}{|c|}{ 95\% C.I. } & P- \\
\hline \multirow[t]{14}{*}{ Ped. } & $\begin{array}{l}\text { Loc. } \\
\text { during } \\
\text { the } \\
\text { crash }\end{array}$ & crosswalk & $\begin{array}{c}\text { At } \\
\text { intersectio } \\
\text { n-outside } \\
\text { crosswalk }\end{array}$ & 1.90 & 1.22 & 2.95 & 0.00 \\
\hline & & & $\begin{array}{c}\text { At } \\
\text { intersectio } \\
\text { n-in } \\
\text { roadway } \\
\end{array}$ & 1.71 & 1.17 & 2.51 & 0.01 \\
\hline & & & $\begin{array}{c}\text { Not at } \\
\text { intersectio } \\
\text { n-in } \\
\text { roadway }\end{array}$ & 3.72 & 2.98 & 4.65 & 0.00 \\
\hline & & & $\begin{array}{c}\text { On } \\
\text { shoulder }\end{array}$ & 2.00 & 1.19 & 3.35 & 0.01 \\
\hline & & & $\begin{array}{l}\text { Beyond } \\
\text { shoulder, } \\
\text { but within } \\
\text { traffic way }\end{array}$ & 2.03 & 1.03 & 3.98 & 0.04 \\
\hline & & & $\begin{array}{c}\text { On } \\
\text { sidewalk }\end{array}$ & 1.26 & 0.50 & 3.19 & 0.60 \\
\hline & & & $\begin{array}{c}\text { Outside } \\
\text { traffic } \\
\text { boundaries }\end{array}$ & 1.66 & 0.74 & 3.76 & 0.20 \\
\hline & & & $\begin{array}{c}\text { Inside } \\
\text { midblock } \\
\text { crossing }\end{array}$ & 0.69 & 0.31 & 1.51 & 0.40 \\
\hline & $\begin{array}{l}\text { Alcohol } \\
\text { Intox. }\end{array}$ & No & Yes & 4.37 & 3.38 & 5.65 & 0.00 \\
\hline & $\begin{array}{l}\text { Vehicle } \\
\text { mov. }\end{array}$ & Straight & $\begin{array}{l}\text { Turning } \\
\text { right }\end{array}$ & 0.30 & 0.24 & 0.39 & 0.00 \\
\hline & & & $\begin{array}{c}\text { Turning } \\
\text { left }\end{array}$ & 0.34 & 0.27 & 0.42 & 0.00 \\
\hline & & & $\begin{array}{l}\text { Stopped in } \\
\text { traffic }\end{array}$ & 0.58 & 0.24 & 1.41 & 0.20 \\
\hline & & & $\begin{array}{l}\text { Parked } \\
\text { properly }\end{array}$ & 0.34 & 0.18 & 0.62 & 0.00 \\
\hline & $\begin{array}{l}\text { Vehicle } \\
\text { type }\end{array}$ & $\begin{array}{l}\text { Passenger } \\
\text { vehicle }\end{array}$ & $\begin{array}{c}\text { Trucks } \\
\text { and buses }\end{array}$ & 2.85 & 1.70 & 4.79 & 0.00 \\
\hline
\end{tabular}


TABLE 49: Single variable model - crash characteristics (odd ratios), continued

\begin{tabular}{|c|c|c|c|c|c|c|c|}
\hline \multirow[t]{12}{*}{ Bic. } & \multirow[t]{7}{*}{$\begin{array}{l}\text { Loc. } \\
\text { during } \\
\text { the } \\
\text { crash }\end{array}$} & \multirow[t]{7}{*}{ crosswalk } & $\begin{array}{c}\text { At } \\
\text { intersectio } \\
\text { n-outside } \\
\text { crosswalk }\end{array}$ & 1.14 & 0.86 & 1.51 & 0.36 \\
\hline & & & $\begin{array}{c}\mathrm{At} \\
\text { intersectio } \\
\mathrm{n} \text {-in } \\
\text { roadway } \\
\end{array}$ & 0.79 & 0.54 & 1.14 & 0.21 \\
\hline & & & $\begin{array}{c}\text { Not at } \\
\text { intersectio } \\
n-\text {-in } \\
\text { roadway }\end{array}$ & 1.92 & 1.23 & 2.98 & 0.00 \\
\hline & & & $\begin{array}{c}\text { On } \\
\text { shoulder }\end{array}$ & 7.53 & 3.32 & 17.0 & 0.00 \\
\hline & & & $\begin{array}{l}\text { Beyond } \\
\text { shoulder, } \\
\text { but within } \\
\text { traffic way }\end{array}$ & 0.97 & 0.41 & 2.33 & 0.95 \\
\hline & & & $\begin{array}{l}\text { In bike } \\
\text { path or } \\
\text { parking } \\
\text { lane }\end{array}$ & 0.90 & 0.43 & 1.89 & 0.79 \\
\hline & & & $\begin{array}{c}\text { In bike } \\
\text { lane }\end{array}$ & 0.53 & 0.18 & 1.51 & 0.23 \\
\hline & $\begin{array}{l}\text { Alcohol } \\
\text { Intox. }\end{array}$ & No & Yes & 2.92 & 1.65 & 5.16 & 0.00 \\
\hline & \multirow[t]{3}{*}{$\begin{array}{l}\text { Vehicle } \\
\text { mov. }\end{array}$} & \multirow[t]{3}{*}{ Straight } & $\begin{array}{l}\text { Turning } \\
\text { right }\end{array}$ & 0.47 & 0.37 & 0.61 & 0.00 \\
\hline & & & $\begin{array}{c}\text { Turning } \\
\text { left }\end{array}$ & 0.49 & 0.35 & 0.67 & 0.00 \\
\hline & & & $\begin{array}{l}\text { Stopped in } \\
\text { traffic }\end{array}$ & 0.25 & 0.13 & 0.48 & 0.00 \\
\hline & $\begin{array}{l}\text { Vehicle } \\
\text { type }\end{array}$ & $\begin{array}{l}\text { Passenger } \\
\text { vehicle }\end{array}$ & $\begin{array}{c}\text { Trucks } \\
\text { and buses }\end{array}$ & 3.28 & 1.12 & 9.63 & 0.03 \\
\hline
\end{tabular}

\subsubsection{Demographic characteristics}

In the event of a crash, (>55) older pedestrians and bicyclists tended to have a higher probability of getting involved in a fatal crash (p-value < 0.05 ). In terms of gender, the findings suggested that male pedestrians had higher odds of being in a fatal crash than 
women at a significant level. For the bicyclist model there was not a statistically significant difference.

TABLE 50: Single variable model - demographic characteristics (odd ratios)

\begin{tabular}{|c|c|c|c|c|c|c|c|}
\hline \multirow[b]{2}{*}{ Ped } & \multirow{2}{*}{$\begin{array}{c}\text { VAR. } \\
\text { Age }\end{array}$} & \multirow{2}{*}{$\begin{array}{c}\begin{array}{c}\text { BASE } \\
\text { GROUP }\end{array} \\
0-14\end{array}$} & \multirow{2}{*}{$\begin{array}{c}\begin{array}{c}\text { LEVE } \\
\text { L }\end{array} \\
15-24\end{array}$} & \multirow{2}{*}{$\begin{array}{c}\begin{array}{c}\text { MEA } \\
\mathbf{N}\end{array} \\
1.17\end{array}$} & \multicolumn{2}{|c|}{ 95\% C.I. } & \multirow{2}{*}{$\begin{array}{c}\begin{array}{c}\text { P- } \\
\text { VALUE }\end{array} \\
0.30\end{array}$} \\
\hline & & & & & 0.87 & 1.58 & \\
\hline . & & & $25-34$ & 1.25 & 0.89 & 1.74 & 0.20 \\
\hline & & & $35-44$ & 1.22 & 0.86 & 1.72 & 0.30 \\
\hline & & & $45-54$ & 1.77 & 1.28 & 2.46 & 0.00 \\
\hline & & & $55-64$ & 1.56 & 1.09 & 2.23 & 0.02 \\
\hline & & & $65-74$ & 1.90 & 1.25 & 2.87 & 0.00 \\
\hline & & & $75-84$ & 3.59 & 2.10 & 6.16 & 0.00 \\
\hline & & & $>84$ & 3.70 & 1.57 & 8.69 & 0.00 \\
\hline & Gender & Male & Female & 0.78 & 0.65 & 0.93 & 0.01 \\
\hline \multirow[t]{9}{*}{ Bic. } & Age & $0-14$ & $15-24$ & 1.32 & 0.93 & 1.88 & 0.12 \\
\hline & & & $25-34$ & 1.45 & 0.99 & 2.10 & 0.05 \\
\hline & & & $35-44$ & 1.27 & 0.85 & 1.90 & 0.25 \\
\hline & & & $45-54$ & 1.38 & 0.94 & 2.03 & 0.10 \\
\hline & & & $55-64$ & 2.11 & 1.31 & 3.39 & 0.00 \\
\hline & & & $65-74$ & 2.99 & 1.60 & 5.60 & 0.00 \\
\hline & & & $75-84$ & 10.11 & 3.72 & 27.4 & 0.00 \\
\hline & & & $>84$ & 33.85 & 1.62 & 707. & 0.02 \\
\hline & Gender & Female & Male & 1.03 & 0.80 & 1.34 & 0.81 \\
\hline
\end{tabular}

\subsubsection{Traffic conditions}

AADT was the only variable available to assess traffic conditions. The results suggested that only AADT for trucks was significant for pedestrian crashes. As truck AADT increased, the odds of a fatal crash increased as well. This result is associated with heavy vehicles, which can cause fatal or severe injuries even if travelling at slow speeds.

In terms of the bicyclist model, the results revealed that AADT decreased the severity of the crashes. Since in this case this variable includes all kind of vehicles, the results make sense. High volumes resulted in less vehicles traveling at higher speeds. 
TABLE 51: Single variable model - traffic conditions (odd ratios)

\begin{tabular}{|c|c|c|c|c|c|c|c|}
\hline & VAR. & BASE & LEVEL & MEA & \multicolumn{2}{|c|}{ 95\% C.I. } & P- \\
\hline \multirow[t]{4}{*}{ Ped. } & \multirow{4}{*}{$\begin{array}{l}\text { Traf. } \\
\text { cond. }\end{array}$} & \multirow[t]{2}{*}{ NA } & AADT & 0.99 & 0.91 & 1.09 & 0.93 \\
\hline & & & $\log (\mathrm{AADT})$ & 0.95 & 0.83 & 1.09 & 0.47 \\
\hline & & \multirow[t]{2}{*}{ NA } & $\begin{array}{l}\text { AADT(only } \\
\text { truck) }\end{array}$ & 1.00 & 0.99 & 1.00 & 0.12 \\
\hline & & & $\begin{array}{c}\log (\mathrm{AADT} \\
\text { truck) }\end{array}$ & 1.18 & 1.04 & 1.34 & 0.01 \\
\hline \multirow[t]{4}{*}{ Bic. } & \multirow{4}{*}{$\begin{array}{l}\text { Traf. } \\
\text { cond. }\end{array}$} & \multirow[t]{2}{*}{ NA } & AADT & 0.88 & 0.79 & 0.98 & 0.02 \\
\hline & & & $\log (\mathrm{AADT})$ & 0.82 & 0.71 & 0.94 & 0.00 \\
\hline & & \multirow[t]{2}{*}{ NA } & $\begin{array}{l}\text { AADT(only } \\
\text { truck) }\end{array}$ & 1.00 & 1.00 & 1.00 & 0.60 \\
\hline & & & $\begin{array}{c}\log (\mathrm{AADT} \\
\text { truck) }\end{array}$ & 0.86 & 0.75 & 0.98 & 0.29 \\
\hline
\end{tabular}

\subsubsection{Road characteristics}

To assess road characteristics we considered number of lanes, width, road classification, road surface, traffic control device and posted speed limit.

Nor number of lanes or road width showed a significant difference for pedestrian and bicyclist crashes. Nonetheless, in terms of road classification (which can be a surrogate for number of lanes), the results revealed that crashes that occurred on arterials were more severe than pedestrian crashes on local streets.

In terms of road surface, the findings suggested that wet road surfaces decreased severity levels compared to dry surfaces. This results is counterintuitive and may be produced by its correlation with weather conditions, which is assessed in section 7.2. In terms of traffic control device, we compared pedestrian crashes based on special pedestrian signal to other devices. Results are not significant; however, they showed that 
the presence of traffic control devices (e.g. medians, traffic signal, or RRFB increased the severity of the crashes. For bicyclist crashes, we compared the presence of control versus no control, but the results are not statistically significant. Finally, pedestrian crash severity increased as posted speed limit increased at a significant level.

TABLE 52: Single variable model - road characteristics (odd ratios)

\begin{tabular}{|c|c|c|c|c|c|c|c|}
\hline & VAR. & BASE & LEVEL & MEAN & \multicolumn{2}{|c|}{ 95\% C.I. } & P-VALUE \\
\hline \multirow[t]{11}{*}{$\begin{array}{c}\text { Ped } \\
.\end{array}$} & $\begin{array}{c}\text { \# of } \\
\text { lanes }\end{array}$ & NA & \# of lanes & 1.07 & 0.98 & 1.17 & 0.15 \\
\hline & Width & NA & Width (ft.) & 1.00 & 0.99 & 1.01 & 0.26 \\
\hline & $\begin{array}{l}\text { Road } \\
\text { class. }\end{array}$ & $\begin{array}{l}\text { Principal } \\
\text { Arterials }\end{array}$ & $\begin{array}{l}\text { Minor } \\
\text { arterial }\end{array}$ & 1.05 & 0.80 & 1.37 & 0.72 \\
\hline & & & $\begin{array}{l}\text { Rural } \\
\text { collector }\end{array}$ & 1.20 & 0.55 & 2.64 & 0.64 \\
\hline & & & $\begin{array}{l}\text { Urban } \\
\text { collector }\end{array}$ & 0.78 & 0.43 & 1.42 & 0.42 \\
\hline & & & Local & 0.51 & 0.29 & 0.89 & 0.01 \\
\hline & Road & Dry & Wet & 0.77 & 0.63 & 0.95 & 0.01 \\
\hline & surface & & Snow & 0.39 & 0.14 & 1.13 & 0.08 \\
\hline & $\begin{array}{l}\text { Traffic } \\
\text { control } \\
\text { device }\end{array}$ & Others & $\begin{array}{c}\text { Special } \\
\text { pedestrian } \\
\text { signal }\end{array}$ & 1.17 & 0.70 & 1.97 & 0.55 \\
\hline & PSL & NA & (mph) & 1.04 & 1.03 & 1.05 & 0.00 \\
\hline & & & $\left(m p h^{2}\right)$ & 1.00 & 1.00 & 1.00 & 0.00 \\
\hline
\end{tabular}


TABLE 52: Single variable model - road characteristics (odd ratios), continued

\begin{tabular}{|c|c|c|c|c|c|c|c|}
\hline \multirow[t]{10}{*}{ Bic. } & $\begin{array}{l}\text { \# of } \\
\text { lanes }\end{array}$ & NA & \# of lanes & 0.91 & 0.82 & 1.01 & 0.07 \\
\hline & Width & NA & Width (ft.) & 0.99 & 0.98 & 1.00 & 0.12 \\
\hline & $\begin{array}{l}\text { Road } \\
\text { class. }\end{array}$ & $\begin{array}{l}\text { Principal } \\
\text { Arterials }\end{array}$ & $\begin{array}{l}\text { Minor } \\
\text { arterial }\end{array}$ & 1.17 & 0.86 & 1.61 & 0.32 \\
\hline & & & $\begin{array}{l}\text { Rural } \\
\text { collector }\end{array}$ & 1.23 & 0.51 & 2.96 & 0.64 \\
\hline & & & & 0.89 & 0.46 & 1.73 & 0.74 \\
\hline & & & Local & 0.74 & 0.43 & 1.27 & 0.28 \\
\hline & $\begin{array}{c}\text { Road } \\
\text { surface }\end{array}$ & Dry & Wet & 0.63 & 0.45 & 0.85 & 0.00 \\
\hline & $\begin{array}{l}\text { Traffic } \\
\text { control } \\
\text { device }\end{array}$ & Others & $\begin{array}{l}\text { Traffic } \\
\text { control } \\
\text { device }\end{array}$ & 1.00 & 0.63 & 1.16 & 1.00 \\
\hline & PSL & NA & (mph) & 1.01 & 0.99 & 1.02 & 0.31 \\
\hline & & & $\left(\mathrm{mph}^{2}\right)$ & 1.00 & 1.00 & 1.00 & 0.36 \\
\hline
\end{tabular}

\subsection{Pooled models}

Pooled models, i.e. with several variables by category (TABLE 55), were used to identify the key risk factors of crash severity. The final models were selected by using the backwards stepwise method based on the Akaike Information Criterion AIC (a forward stepwise was also used, but the final variables were the same). Once the key risk factors were identified, we dropped the ones that were not significant and included them in the base group category. A Additionally, we did not include the observations of interstate and freeways, since pedestrians and bicyclists are not allowed to be in these facilities. Furthermore, we interacted factors that were correlated for a better model estimation and interpretation. Finally, we tested for the proportional odds assumption to assess the models. For the pedestrian model, we found that posted speed limit and AADT 
(only truck) did not pass the test. We classified the speed variable into two categories, $<50 \mathrm{mph}$ and $\geq 50 \mathrm{mph}$. For AADT (only truck) we created two categories using the $25 \%$ percentile of the variable distribution as the cut-point (TABLE 53) to differentiate between low and high heavy vehicle volume. By doing this, the proportional odds test was met. Final models are shown in TABLE 55 (for more detail see (TABLE 85). Parameters are in log odds units. Additionally, odd ratios were estimated for a better interpretation of the results (FIGURE 6).

TABLE 53: AADT only trucks cut-points

\begin{tabular}{c|c}
\hline \hline AADT only trucks (cut-points) & Observations \\
\hline$\leq 670$ & 390 \\
\hline$>670$ & 1145 \\
\hline \hline
\end{tabular}

For the pedestrian model, the findings suggested that age, followed by alcohol intoxication, had the biggest impact on crash severity prediction. In a crash event, older pedestrians were at a higher risk than young pedestrians ( $\mathrm{p}$-value $<0.001)$. The odds of a fatal $(\mathrm{K})$ crash involving a person older than 74 versus the other severity levels combined, were 4.91 times that of young pedestrians ( $\mathrm{p}$-value $<0.001)$ given that all the other variables in the model remain constant. For pedestrians between 55 and 74, the odds were 1.64 greater. If a crash involves an alcohol intoxicated driver, the odds of a fatal $(\mathrm{K})$ crash versus the other severity levels combined were 2.48 times that of sober drivers, ceteris paribus ( $\mathrm{p}$-value $<0.001)$.

In terms of environmental conditions, only light condition was included in the model due to its statistical significant level. We separated darkness with no streetlight from the other lighting conditions. The findings suggested that if a crash occurs in dark 
conditions with no streetlights, the odds of a fatal $(\mathrm{K})$ crash versus the other severity levels combined were 1.95 times that of other lighting conditions ( $\mathrm{p}$-value $<0.001$ ).

Crash characteristics were also significant predictors of severity. The odds of a fatal crash where a truck (or bus) was involved were 2.48 times that of passenger vehicles (p-value $<0.001)$. Furthermore, the odds of a fatal crash where a vehicle was turning were 0.49 times that of a vehicle travelling straight ( $\mathrm{p}$-value $<0.001$ ). Vehicles turning tend to decrease their speed, which result in less severe crashes.

Considering road characteristics, we found that local streets, special pedestrian traffic control device and posted speed limits were statistically significant. The odds of a fatal crash in a local street were half that of a crash in arterials ( $p$-value $<0.05)$. Highposted speed limits tended to increase crash severity as well. The odds of a fatal $(\mathrm{K})$ crash versus the other severity levels combined in a $\geq 50 \mathrm{mph}$ road were 1.60 times that of $<50$ mph roads.

In terms of traffic conditions, the findings suggested that AADT for trucks was a statistically significant predictor for crash severity. The odds of a fatal crash in a road with an AADT (only trucks) higher than 670 heavy vehicles were 1.36 times that of a road with an AADT below 670 heavy vehicles.

Traffic control device indicates the predominant control present at the crash location. If more than one control was present, the crash recorder chose the device that was more related to the crash. We explored different devices and found that the presence of traffic control devices (i.e. Rectangular Rapid Flash Beacon-RRFB, median, traffic signal) was significant in the pooled model. The findings suggested that the odds of a crash where a control device was present, was 2.07 times that of other control devices. 
This result must be taken with caution because it must be the result of poor data quality (incorrect coding). Special pedestrian signals such as RRFBs have been found to reduce crash frequency (Monsere, Figliozzi, Kothuri, Razmpa, \& Hazel, 2016). Monsere et al. (2016) identified a trend in the reduction of severity of pedestrian crashes after the installation of several crosswalk treatments (RRFB, flashing amber, high visibility and standard parallel crossings); a shift from level $\mathrm{K}$ and $\mathrm{A}$, to B and $\mathrm{C}$.

We found several potential explanations for our finding. First, the installation of these devices may increase the pedestrian volume, increasing crash frequency. Furthermore, these special signals are normally installed at locations with high crash frequency, which can reduce crash frequency but may not have an impact on severity when a crash occurs. Finally, pedestrians may feel more confident that incoming traffic would yield to pedestrians if for example an RRFB has been activated or a median is present. Another reason for the significance of this variable was also found on how this factor is recorded in the crash database. At the time of filling out the crash report, there are not enough instructions on what is a special pedestrian signal, which may cause errors in the report.

Finally, an interaction variable between weather and road surface conditions was added due to the correlation between the two variables (i.e. wet road conditions and rainy day). The odds of a fatal crash on a wet road during a clear day were 1.54 that of other weather and road surface conditions.

Location, vehicle movement, age, alcohol intoxication, AADT, and light conditions were significant in the crash model for bicyclists. The AADT did not meet the proportion odds assumption, for this reason we transformed this variable into categorical. 
Tot try to capture roads with low and high volume, we used the $25 \%$ percentile of the AADT distribution as the cut-point (10,000 vehicles). See TABLE 54 for more detail. Doing this allowed us to meet the assumption.

TABLE 54: AADT cut-points

\begin{tabular}{c|c}
\hline \hline AADT only trucks (cut-points) & Observations \\
\hline$\leq 10,000$ & 222 \\
\hline$>10,000$ & 778 \\
\hline \hline
\end{tabular}

The odds of a fatal crash in a roadway segment versus the other severity levels combined were 1.77 times that of a crash at an intersection. For crash characteristics the results were similar to the pedestrian model, vehicles turning tended to be less severe than crashes with vehicles going straight (odds of 0.66, p-value<0.01).

In terms of age, The odds of a fatal $(\mathrm{K})$ crash involving a person older than 74 versus the other severity levels combined, were 9.29 times that of young pedestrians (pvalue $<0.001)$ given that all the other variables in the model remain constant. For pedestrians between 55 and 74, the odds were 1.91 greater.

The result for AADT was intuitive, more cars were found to decrease the likelihood of a crash being fatal. High volumes may result in a high crash frequency but less fatal and severe crashes since vehicles are not travelling fast. The odds of a fatal crash in a road with an AADT above 10,000 vehicles per day were 0.74 that of roads below 10,000 vehicles.

Alcohol intoxication was only significant at the 0.1 level, for this reason we explored this variable in terms of others, such as light conditions. Lee and Abdel-Aty (2003) explored the interaction between lighting conditions and impaired drivers and 
found that people who were driving under alcohol effects in dark conditions, tended to be involved in more severe crashes than people driving during other lighting conditions. We found similar evidence. The odds of fatal crashes involving an alcohol-intoxicated driver and during dark conditions were 3.81 times that of crashes where the driver was sober or the driver was intoxicated but not driving at night.

TABLE 55: Ordinal regression model. Crash severity for pedestrians and bicyclists

\begin{tabular}{|c|c|c|c|c|c|c|}
\hline VARIABLE & BASE & LEVEL & \multicolumn{2}{|c|}{$\begin{array}{c}\text { PED. } \\
\text { PARAM. }\end{array}$} & \multicolumn{2}{|c|}{$\begin{array}{c}\text { BIC. } \\
\text { PARAM. }\end{array}$} \\
\hline $\begin{array}{c}\text { Light } \\
\text { conditions }\end{array}$ & $\begin{array}{l}\text { Other lighting } \\
\text { conditions }\end{array}$ & $\begin{array}{l}\text { Darkness no } \\
\text { street light }\end{array}$ & 0.67 & $* * *$ & NA & \\
\hline $\begin{array}{c}\text { Alcohol } \\
\text { intoxication }\end{array}$ & No & Yes & 0.91 & $* * *$ & NA & \\
\hline $\begin{array}{c}\text { Vehicle } \\
\text { movement }\end{array}$ & Straight & Turning & -0.72 & $* * *$ & -0.41 & $* *$ \\
\hline Vehicle type & $\begin{array}{l}\text { Passenger } \\
\text { vehicle }\end{array}$ & $\begin{array}{c}\text { Truck and } \\
\text { buses }\end{array}$ & 0.91 & $* *$ & NA & \\
\hline $\begin{array}{c}\text { Road } \\
\text { classification }\end{array}$ & Arterials & $\begin{array}{l}\text { Local } \\
\text { streets }\end{array}$ & -0.74 & $*$ & NA & \\
\hline $\begin{array}{c}\text { Traffic } \\
\text { control } \\
\text { Device }\end{array}$ & Other devices & $\begin{array}{c}\text { Special } \\
\text { pedestrian } \\
\text { signal }\end{array}$ & 0.73 & $* *$ & NA & \\
\hline \multirow[t]{2}{*}{ Age } & $\leq 54$ & $55-74$ & 0.50 & $* * *$ & 0.65 & $* * *$ \\
\hline & & $>74$ & 1.60 & $* * *$ & 2.23 & $* * *$ \\
\hline $\begin{array}{l}\text { Posted speed } \\
\text { limit }\end{array}$ & $<50 \mathrm{mph}$ & $\geq 50 \mathrm{mph}$ & 0.46 & $*$ & NA & \\
\hline Location & Intersection & Segment & NA & & 0.57 & $* *$ \\
\hline \multirow{2}{*}{$\begin{array}{l}\text { Ped. /Bic. } \\
\text { location } \\
\text { during the } \\
\text { crash }\end{array}$} & Crosswalk & Roadway & 0.36 & $* *$ & NA & \\
\hline & & Midblock & -0.86 & $* *$ & NA & \\
\hline $\begin{array}{c}\text { AADT (only } \\
\text { truck) }\end{array}$ & $<670$ & $670-1100$ & 0.31 & $* *$ & & \\
\hline AADT & $\leq 10,000$ & $>10,000$ & NA & & -0.31 & $*$ \\
\hline
\end{tabular}


TABLE 55: Ordinal regression model. Crash severity for pedestrians and bicyclists, continued

\begin{tabular}{|c|c|c|c|c|c|c|c|}
\hline \multirow{2}{*}{\multicolumn{2}{|c|}{$\begin{array}{c}\text { VARIABLE } \\
\text { Interaction } \\
\text { weather and } \\
\text { road surface } \\
\text { conditions } \\
\end{array}$}} & \multirow{2}{*}{$\begin{array}{c}\text { BASE } \\
\text { GROUP } \\
\text { Other weather } \\
\text { and road } \\
\text { surface } \\
\text { conditions } \\
\end{array}$} & \multirow{2}{*}{$\begin{array}{c}\text { LEVEL } \\
\text { Wet road } \\
\text { surface and } \\
\text { clear day }\end{array}$} & \multicolumn{2}{|c|}{$\begin{array}{c}\text { PED. } \\
\text { PARAM. }\end{array}$} & \multicolumn{2}{|c|}{$\begin{array}{c}\text { BIC. } \\
\text { PARAM. }\end{array}$} \\
\hline & & & & 0.43 & $* *$ & NA & \\
\hline \multirow{6}{*}{\multicolumn{2}{|c|}{$\begin{array}{c}\text { Interaction } \\
\text { light } \\
\text { conditions } \\
\text { and alcohol } \\
\text { intoxication } \\
\end{array}$}} & $\begin{array}{l}\text { Other light } \\
\text { and alcohol } \\
\text { intoxication } \\
\text { conditions }\end{array}$ & $\begin{array}{l}\text { Alcohol } \\
\text { intoxication } \\
\text { during dark } \\
\text { conditions }\end{array}$ & NA & & 1.34 & $* *$ \\
\hline & & $\begin{array}{c}\text { Threshold } \\
\text { coefficients }\end{array}$ & & & & & \\
\hline & & & $\mathrm{O} \mid \mathrm{C}$ & -4.80 & $* * *$ & -3.85 & $* * *$ \\
\hline & & & $\mathrm{C} \mid \mathrm{B}$ & -0.42 & $* * *$ & -1.02 & $* * *$ \\
\hline & & & $\mathrm{B} \mid \mathrm{A}$ & 1.73 & $* * *$ & 2.18 & $* * *$ \\
\hline & & & $\mathrm{A} \mid \mathrm{K}$ & 3.07 & $* * *$ & 3.88 & $* * *$ \\
\hline$*$ & 0.01 & $\leq$ p-value $<0.05$ & Log. Lik. & -1800.98 & & -1011.39 & \\
\hline$* *$ & 0.001 & $\leq$ p-value $<0.01$ & AIC & 3635.96 & & 2042.78 & \\
\hline$* * *$ & 0 & $\leq$ p-value $<0.001$ & & & & & \\
\hline NA & not app & plicable & & & & & \\
\hline
\end{tabular}




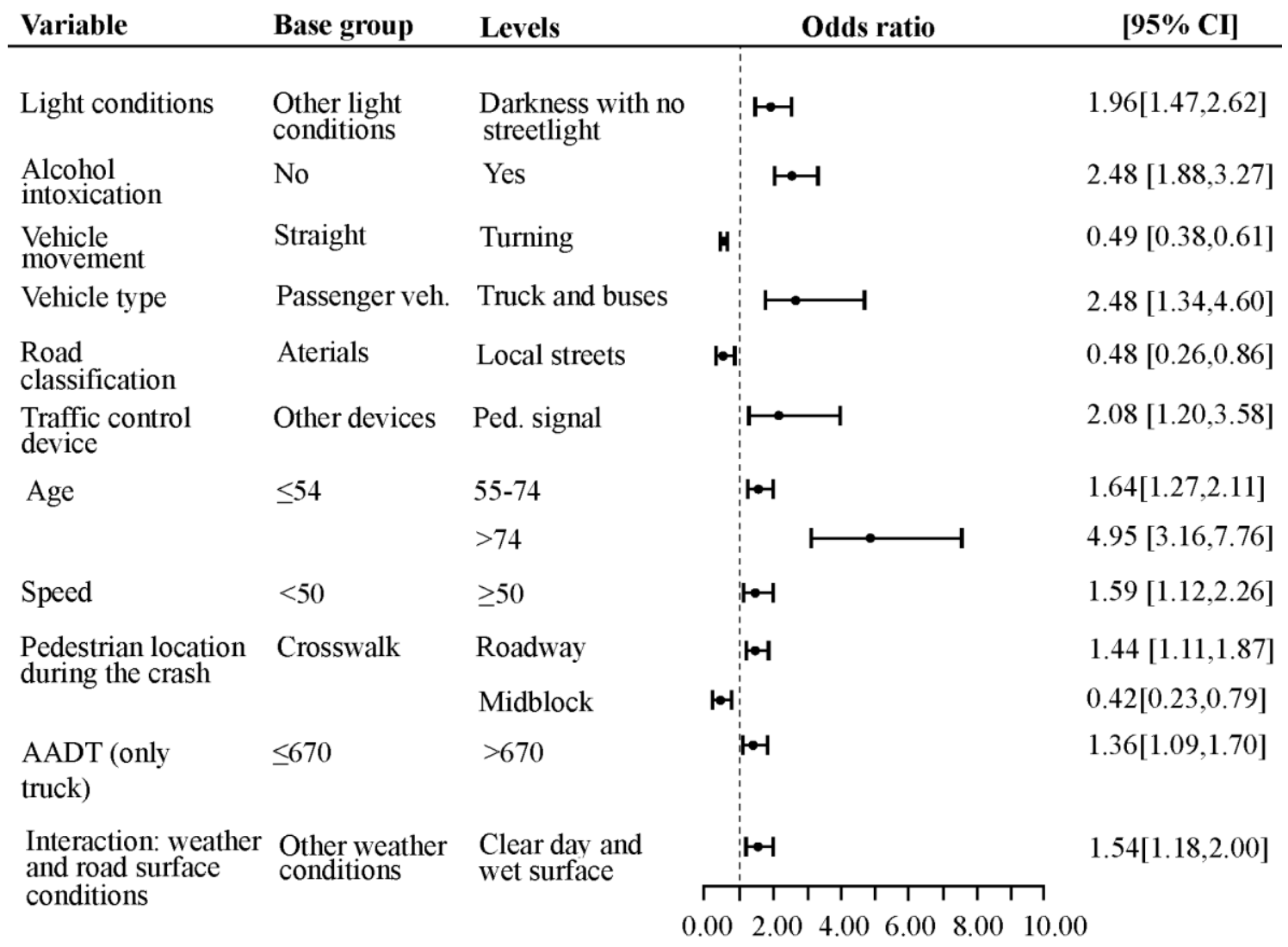

(a)

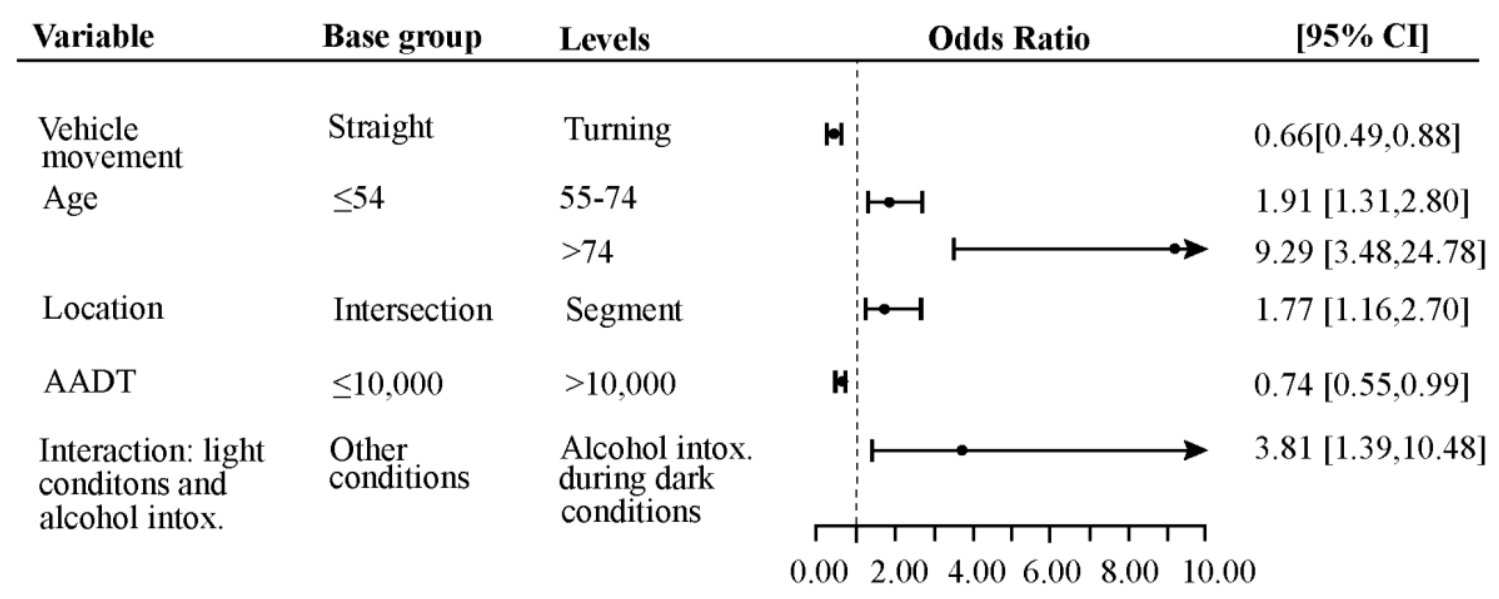

(b)

FIGURE 6 Odd ratios for (a) Pedestrian and (b) Bicyclist models 
Finally, we explored which risk factors had the biggest predictive power in the final model. By removing each variable at a time (ceteris paribus), we obtained the difference in log likelihood between the full model and the model with one variable removed. Then, we ranked the variables from the highest log likelihood difference to the lowest (TABLE 56).

TABLE 56: Predictive power rank

\begin{tabular}{|c|c|c|c|c|}
\hline \multirow[b]{2}{*}{ RANK } & \multicolumn{2}{|c|}{ PEDESTRIAN MODEL } & \multicolumn{2}{|c|}{ BICYCLIST MODEL } \\
\hline & VARIABLE & $\begin{array}{c}\text { Log } \\
\text { Likelihood } \\
\text { difference }\end{array}$ & VARIABLE & $\begin{array}{c}\text { Log } \\
\text { Likelihood } \\
\text { difference }\end{array}$ \\
\hline 1 & Age & 29.17 & Age & 14.02 \\
\hline 2 & Alcohol intoxication & 20.98 & Vehicle movement & 3.91 \\
\hline 3 & Vehicle movement & 18.22 & Location & 3.53 \\
\hline 4 & Light conditions & 10.39 & $\begin{array}{c}\text { Interaction light } \\
\text { conditions and } \\
\text { alcohol } \\
\text { intoxication }\end{array}$ & 3.19 \\
\hline 5 & $\begin{array}{l}\text { Pedestrian/Bicyclist } \\
\text { location during the crash }\end{array}$ & 8.81 & AADT & 2.07 \\
\hline 6 & $\begin{array}{l}\text { Interaction weather and } \\
\text { road surface conditions }\end{array}$ & 5.15 & & \\
\hline 7 & Vehicle type & 4.16 & & \\
\hline 8 & AADT(only truck) & 3.91 & & \\
\hline 9 & Traffic control device & 3.38 & & \\
\hline 10 & Posted speed limit & 3.30 & & \\
\hline 11 & Road classification & 3.14 & & \\
\hline
\end{tabular}

Overall, for both models age was the strongest risk factor. For the pedestrian model, the most important risk factors after age were related to cash characteristics (alcohol intoxication, and vehicle movement). Light conditions also had a strong predictive power for crash severity. In terms of the bicyclist model, vehicle movement was the second strongest variable; however, the log likelihood difference is one third of the difference of age. 


\subsection{Road characteristics and traffic conditions models}

Exploring location, environmental, crash, demographic, traffic and road characteristics was useful to identify risk factors of crash severity. Nonetheless, it is the interest of DOTs to know risk factors related to road and traffic characteristics, since the agencies will have more room to implement countermeasures and policies to reduce fatalities. This section focuses only on road and traffic characteristics were used to identify risk factors. Similar to the methodology used in section 7.2, we estimated ordinal regression models for pedestrian and bicyclist crashes. We selected the variables by using a backwards stepwise method based on AIC. Results are shown in TABLE 57 (for more detail see TABLE 86). Additionally, for a better interpretation, odd ratios are shown in FIGURE 7.

For the pedestrian model, results are similar to TABLE 55, with the difference that now location of the crash (intersection vs segment) and AADT (only for trucks) are significant. Risk factors that were repeated from the pooled models (TABLE 55) kept the same direction with severity level (sign) and similar coefficients.

We found strong evidence that the odds of a crash of being fatal at an intersection of being fatal were 0.71 times that of a crash that occurred in a segment of the road. In other words, if a crash occurred in a segment of the road, it had a higher probability of being fatal.

Furthermore, we found that volume of trucks had a positive relationship on the likelihood of fatal crashes. In terms of AADT for trucks, the findings suggested that high AADTs increased the likelihood of fatal crashes ( $\mathrm{p}$-value $<0.05$ ). The odds of a crash being fatal in a roadway with and AADT (only trucks) above 670 were 1.47 that of a 
roadway with an AADT below 670 vehicles. This result was consistent with the previous findings, where a crash that involved a heavy vehicle had a greater chance of being fatal. In terms of the bicycle model, we found that only location (intersection vs segment) and AADT are significant risk factors to explain severity. Location was found in the pooled models (TABLE 55) and it maintained the same sign in this model (in relation to the standard deviation).

The odds of a fatal crash in a roadway segment versus the other severity levels combined were 2.03 times that of a crash at an intersection (p-value $<0.01)$. In terms of AADT, the findings suggested that crashes being fatal are less likely to happen in low volume roads than high volume roads. The odds of a crash being fatal in a roadway with an AADT below 10,000 were 0.72 times that of roadways with AADT above 10,000. 
TABLE 57: Ordinal regression model. Road and traffic characteristics

\begin{tabular}{|c|c|c|c|c|c|c|c|}
\hline \multirow{2}{*}{\multicolumn{2}{|c|}{$\begin{array}{c}\text { VARIABLE } \\
\begin{array}{c}\text { Light } \\
\text { conditions }\end{array}\end{array}$}} & \multirow{2}{*}{$\begin{array}{c}\text { BASE } \\
\text { GROUP } \\
\begin{array}{c}\text { Other lighting } \\
\text { conditions }\end{array}\end{array}$} & \multirow{2}{*}{$\begin{array}{c}\text { LEVEL } \\
\text { Darkness no } \\
\text { street light }\end{array}$} & \multicolumn{2}{|c|}{$\begin{array}{c}\text { PED. } \\
\text { PARAM. }\end{array}$} & \multicolumn{2}{|c|}{$\begin{array}{c}\text { BIC. } \\
\text { PARAM. }\end{array}$} \\
\hline & & & & 0.88 & $* * *$ & NA & \\
\hline & Road class. & Arterials & Local streets & -0.76 & $*$ & NA & \\
\hline & PSL & $<50 \mathrm{mph}$ & $\geq 50 \mathrm{mph}$ & 0.47 & $* *$ & NA & \\
\hline & Location & Intersection & Segment & 0.71 & $* * *$ & 0.86 & $* * *$ \\
\hline & $\begin{array}{l}\text { ADT (only } \\
\text { truck) }\end{array}$ & $\leq 670$ & $>670$ & 0.39 & $* * *$ & NA & \\
\hline \multirow{6}{*}{\multicolumn{2}{|c|}{ AADT }} & $\leq 10,000$ & $>10,000$ & NA & & -0.33 & $*$ \\
\hline & & $\begin{array}{c}\text { Threshold } \\
\text { coefficients }\end{array}$ & & & & & \\
\hline & & & $\mathrm{O} \mid \mathrm{C}$ & -4.50 & $* * *$ & -3.66 & $* * *$ \\
\hline & & & $\mathrm{C} \mid \mathrm{B}$ & -0.21 & $* * *$ & -0.85 & $* * *$ \\
\hline & & & $\mathrm{B} \mid \mathrm{A}$ & 1.76 & $* * *$ & 2.22 & $* * *$ \\
\hline & & & \begin{tabular}{l|l}
$\mathrm{A}$ & $\mathrm{K}$ \\
\end{tabular} & 2.99 & $* * *$ & 3.86 & $* * *$ \\
\hline$*$ & $0.01 \leq$ & p-value $<0.05$ & Log. Lik. & -1890.68 & & -1033.67 & \\
\hline$* *$ & $0.001 \leq$ & p-value $<0.01$ & AIC & 3799.36 & & 2079.34 & \\
\hline$* * *$ & $0 \leq$ & p-value $<0.001$ & & & & & \\
\hline NA & not appl & cable & & & & & \\
\hline
\end{tabular}




\begin{tabular}{|c|c|c|c|c|}
\hline Variable & Base group & Levels & Odds ratio & {$[95 \% \mathrm{CI}]$} \\
\hline Light conditions & $\begin{array}{l}\text { Other light } \\
\text { conditions }\end{array}$ & $\begin{array}{l}\text { Darkness with no } \\
\text { streetlight }\end{array}$ & $\mapsto-1$ & $2.40[1.80,3.19]$ \\
\hline $\begin{array}{l}\text { Road } \\
\text { classification }\end{array}$ & Aterials & Local streets & d: & $0.47[0.26,0.84]$ \\
\hline Speed & $<50$ & $\geq 50$ & :-1 & $1.60[1.13,2.25]$ \\
\hline Location & Intersection & Segment & $1-1$ & $2.04[1.64,2.53]$ \\
\hline $\begin{array}{l}\text { AADT (only } \\
\text { truck) }\end{array}$ & $\leq 670$ & $>670$ & |r & $1.47[1.18,1.82]$ \\
\hline
\end{tabular}

(a)

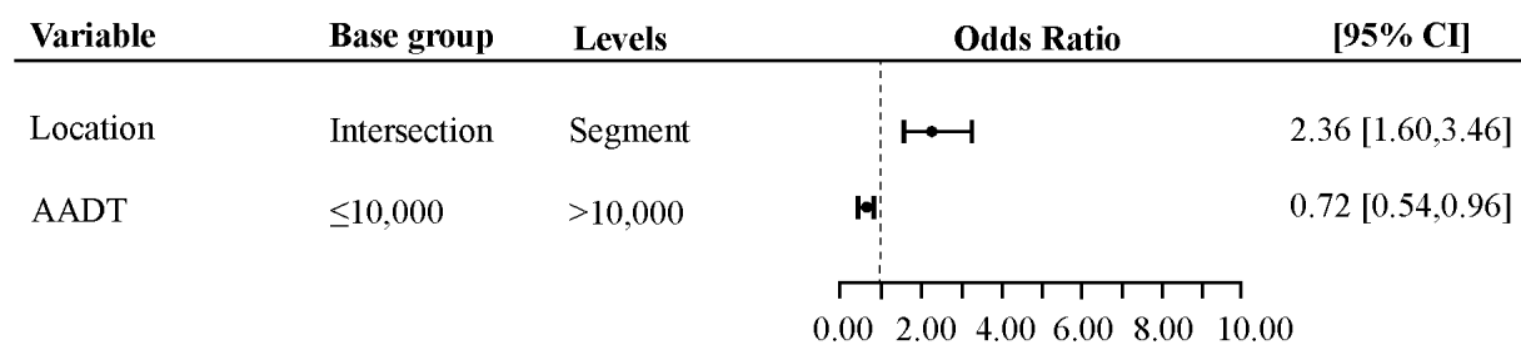

(b)

FIGURE 7 Odd ratios for (a) Pedestrian and (b) Bicyclist models (only road and traffic characteristics)

Similarly to TABLE 56, we explored which risk factors had the biggest predictive power in the final model. TABLE 58 shows road and traffic risk factors ranked by predictive power. Most of the risk factors kept the same order found in TABLE 56. The only difference was found in location of the pedestrian crash, which was not significant in the pooled model.

For both pedestrian and bicyclist models, the location factor was the strongest variable, followed by light conditions and truck AADT (in the pedestrian model), and AADT (in the bicyclist model). 
TABLE 58: Predictive power rank (road and traffic characteristics)

\begin{tabular}{ccccc}
\hline \hline RANK & \multicolumn{2}{c}{ PEDESTRIAN MODEL } & \multicolumn{2}{c}{ BICYCLIST MODEL } \\
& VARIABLE & $\begin{array}{c}\text { Log Likelihood } \\
\text { difference }\end{array}$ & VARIABLE & $\begin{array}{c}\text { Log Likelihood } \\
\text { difference }\end{array}$ \\
\hline 1 & Location & 21.35 & Location & \\
2 & Light conditions & 18.30 & AADT & 2.94 \\
3 & AADT(only truck) & 6.24 & & \\
4 & Posted speed limit & 3.60 & & \\
5 & Road classification & 3.38 & & \\
\hline \hline
\end{tabular}




\section{DISCUSSION}

The results mostly agreed with the literature review. Risk factors associated with high vehicle speeds, visibility, vehicle size, alcohol intoxication, and age of the pedestrian/bicyclist explained crash severity level at a significant level.

From the exposure analysis, we focused only on road characteristics and neighborhood concepts. This analysis was advantageous since it allowed us to identify patterns by controlling for exposure. Overall, the results suggested that as number of lanes and road width increased, pedestrian and bicyclist crash severity tended to increase. High concentration of crashes was found in four lane roads, 40-50 ft. roads, and roads with a posted speed limit between 20 to $25 \mathrm{mph}$. In terms of neighborhood concepts, the findings suggested that high risk ratios were concentrated in suburban areas (concept $\mathrm{E}$ and F). Additionally, most of the fatal crashes in these land uses occurred on segments as opposed to intersections.

The exploration of the variables through the single variable models showed some potential risk factors for crash severity. In the pedestrian model, neighborhood concepts E and F, related to suburban and rural areas respectively, were found to increase severity levels. Nonetheless, we did not find evidence in the pooled or only road and traffic characteristics models suggesting that land use (neighborhood concepts) was related to crash severity. Driver, road and traffic characteristics were found to have a significant stronger association with severity than land use.

Moreover, a look to traffic conditions through the single variable models revealed that truck AADT yielded statistically significant associations with severity. As truck volume increased, the probability of a fatal crash increased. Similar results were found in 
(Kashani \& Besharati, 2016). Other risk factors that had a statistically positive association with crash severity were number of lanes and road width.

For the bicyclist single variable models, results indicated that AADT (for all vehicles) decreased crash severity. This result is consistent with the literature (2004), where we found that even if AADT increased crash frequency, the severity was found to be lower perhaps due to vehicles travelling at a lower speed. Furthermore, it was found that heavy vehicles (trucks and buses) were associated with severe and fatal crashes.

In terms of the pooled models (TABLE 55) and the only road and traffic factors models (TABLE 57), the impact of the risk factors are better understood if they are applied to specific examples. TABLE 59 shows crash severity predictions for different scenarios. For the pedestrian model, we considered the base line to be the safest scenario (young pedestrian, good light conditions, crash occurred at an intersection, driver was sober, passenger vehicle turning, posted speed limit under $50 \mathrm{mph}$, local street, dry road surface, low truck volume, and no traffic control device). The probability for a fatal or incapacitated crash under these conditions is only $3.9 \%$. For the rest of the scenarios, we estimated probabilities based on the worst level of each risk factor, as shown below.

1. Worst case scenario (scenario where all the worst risk factor levels occurred.)

2. Pedestrian older than 74

3. Driver was alcohol intoxicated

4. Truck or bus involved in the crash

5. Crash occurred on an arterial

6. Special pedestrian signal present

7. Vehicle was moving straight 
8. Dark conditions and no street light

9. Posted speed limit over $50 \mathrm{mph}$

10. Wet road surface and clear day

11. The pedestrian was walking on the roadway (as opposed to using a crosswalk) 12. AADT (only trucks) above 670 vehicles

TABLE 59 reveals the change (\%) between each scenario and the base line. For example, in the worst-case scenario, the likelihood that the crash will be fatal or incapacitated injury will increase by $95.1 \%$. Overall, the findings suggested that any change on any of the risk factor levels, increased the probability of severity levels $\mathrm{K}$, A and B. In terms of the most important risk factors, the likelihood of a fatal or incapacitated injury crash increased as pedestrians are older than $74(+12.9 \%)$, the driver was alcohol intoxicated $(+5.3 \%)$, or a truck or bus was involved in the crash $(+5.3 \%)$,

The second part of the sensitivity analysis only considered the bicycle model. In this case, the base line (safest case) was the scenario where a crash occurred at an intersection, the vehicle was turning, the driver was sober under good lighting conditions, high AADT (lower vehicle speeds), and the bicyclist was younger than 55. In this scenario, the probability of being in a fatal or incapacitated injury crash is $5.3 \%$. Five different scenarios were estimated:

1. Worst case scenario (scenario where all the worst risk factor levels occurred.)

2. Pedestrian older than 74

3. Driver was alcohol intoxicated and driving during dark conditions

4. Crash occurred on the roadway as opposed to an intersection

5. Vehicle was moving straight 
6. Low AADT (below 10,000)

As with the pedestrian model results, any change in the risk factors increases the likelihood of fatal and incapacitated injury crashes. If a bicyclist gets into a crash under the worst-scenario conditions, the probability of a $\mathrm{K}+\mathrm{A}$ crash increases by almost $82.5 \%$. Age and an intoxicated driver at night have the strongest effect on crash severity outcomes.

TABLE 59: Impact of risk factors on severity level

\begin{tabular}{|c|c|c|c|c|c|c|}
\hline & PED. MODEL & LEVEL & $\begin{array}{c}\mathbf{K}+\mathbf{A} \\
(\%)\end{array}$ & $\begin{array}{c}\text { B } \\
(\%)\end{array}$ & $\begin{array}{l}\mathrm{C} \\
(\%)\end{array}$ & $\begin{array}{c}\mathbf{O} \\
(\%)\end{array}$ \\
\hline \multirow{13}{*}{ 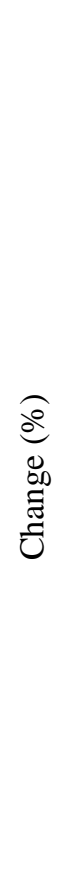 } & Base line (\%) & & 3.9 & 22.1 & 70.6 & 3.4 \\
\hline & Worst case scenario & & 95.1 & -21.3 & -70.5 & -3.4 \\
\hline & Age & $>74$ & 12.9 & 24.5 & -34.6 & -2.7 \\
\hline & Alcohol intoxication & Yes & 5.3 & 15.3 & -18.6 & -2.0 \\
\hline & Vehicle type & $\begin{array}{c}\text { Passenger } \\
\text { vehicle }\end{array}$ & 5.3 & 15.2 & -18.5 & -2.0 \\
\hline & Road classification & Arterials & 4.0 & 12.5 & -14.8 & -1.8 \\
\hline & Device & $\begin{array}{c}\text { Pedestrian } \\
\text { signal }\end{array}$ & 3.9 & 12.2 & -14.4 & -1.7 \\
\hline & Vehicle movement & Straight & 3.8 & 12.1 & -14.3 & -1.7 \\
\hline & Light conditions & $\begin{array}{c}\text { Darkness no } \\
\text { street light }\end{array}$ & 3.5 & 11.2 & -13.1 & -1.6 \\
\hline & Speed & $>=50 \mathrm{mph}$ & 2.2 & 7.7 & -8.7 & -1.3 \\
\hline & $\begin{array}{l}\text { Wet road surface and } \\
\text { clear day }\end{array}$ & & 2.0 & 7.1 & -8.0 & -1.2 \\
\hline & $\begin{array}{l}\text { Pedestrian location } \\
\text { during the crash }\end{array}$ & Roadway & 1.6 & 5.9 & -6.5 & -1.0 \\
\hline & AADT (only trucks) & $>670$ & 1.3 & 4.7 & -5.2 & -0.9 \\
\hline
\end{tabular}


TABLE 59: Impact of risk factors on severity level, continued

\begin{tabular}{|c|c|c|c|c|c|c|}
\hline & BIC. MODEL & LEVEL & $\begin{array}{c}K+A \\
(\%)\end{array}$ & $\begin{array}{c}\text { B } \\
(\%) \\
\end{array}$ & $\begin{array}{c}\mathrm{C} \\
(\%)\end{array}$ & $\begin{array}{c}0 \\
(\%) \\
\end{array}$ \\
\hline \multirow{7}{*}{ 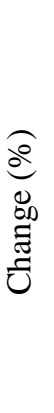 } & Base line (\%) & & 5.3 & 52.3 & 38.3 & 4.2 \\
\hline & Worst case scenario & & 82.5 & -40.5 & -37.8 & -4.1 \\
\hline & Age & $>74$ & 28.8 & 6.4 & -31.4 & -3.7 \\
\hline & $\begin{array}{l}\text { Alcohol intoxication } \\
\text { during dark conditions }\end{array}$ & & 12.2 & 14.1 & -23.2 & -3.0 \\
\hline & Location & Segment & 3.7 & 9.4 & -11.3 & -1.8 \\
\hline & Vehicle movement & Straight & 2.5 & 7.1 & -8.2 & -1.4 \\
\hline & AADT & $<10,000$ & 1.8 & 5.5 & -6.2 & -1.1 \\
\hline
\end{tabular}

A similar analysis was developed for the models with only road and traffic characteristics (TABLE 60). For pedestrian crashes, the most important risk factors are light conditions and road classification. Or bicyclist crashes is location (segment vs intersection).

Providing better lighting conditions at night and separating pedestrian and bicyclist movement in segments of the road and arterials are key strategies DOTs can implement to reduce fatalities. 
TABLE 60: Impact of road and traffic risk factors on severity level

\begin{tabular}{|c|c|c|c|c|c|c|}
\hline & $\begin{array}{c}\text { PED. } \\
\text { MODEL }\end{array}$ & LEVEL & $\begin{array}{c}K+A \\
(\%)\end{array}$ & $\begin{array}{c}\text { B } \\
(\%)\end{array}$ & $\begin{array}{c}\mathrm{C} \\
(\%)\end{array}$ & $\begin{array}{c}\mathbf{O} \\
(\%) \\
\end{array}$ \\
\hline \multirow{8}{*}{ 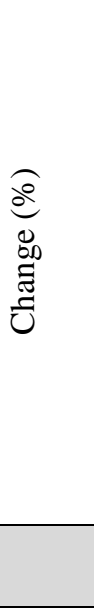 } & $\begin{array}{c}\text { Base line } \\
(\%)\end{array}$ & & $7.4 \%$ & $29.2 \%$ & $61.1 \%$ & $2.3 \%$ \\
\hline & $\begin{array}{c}\text { Worst case } \\
\text { scenario }\end{array}$ & & $58.9 \%$ & $-2.1 \%$ & $-54.6 \%$ & $-2.2 \%$ \\
\hline & $\begin{array}{c}\text { Light } \\
\text { conditions }\end{array}$ & $\begin{array}{c}\text { Darkness no } \\
\text { street light }\end{array}$ & $8.7 \%$ & $12.8 \%$ & $-20.1 \%$ & $-1.3 \%$ \\
\hline & $\begin{array}{c}\text { Road } \\
\text { classification }\end{array}$ & Arterials & $7.2 \%$ & $11.4 \%$ & $-17.4 \%$ & $-1.2 \%$ \\
\hline & Location & Segment & $6.6 \%$ & $10.8 \%$ & $-16.3 \%$ & $-1.2 \%$ \\
\hline & Speed & $>=50 \mathrm{mph}$ & $3.9 \%$ & $7.4 \%$ & $-10.5 \%$ & $-0.8 \%$ \\
\hline & $\begin{array}{c}\text { AADT (only } \\
\text { trucks) }\end{array}$ & $>670$ & $3.1 \%$ & $6.2 \%$ & $-8.6 \%$ & $-0.7 \%$ \\
\hline & $\begin{array}{c}\text { BIC. } \\
\text { MODEL }\end{array}$ & LEVEL & $\begin{array}{c}K+A \\
(\%)\end{array}$ & $\begin{array}{c}\text { B } \\
(\%)\end{array}$ & $\begin{array}{c}\mathrm{C} \\
(\%)\end{array}$ & $\begin{array}{c}0 \\
(\%)\end{array}$ \\
\hline \multirow{4}{*}{ 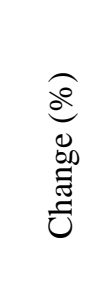 } & $\begin{array}{c}\text { Base line } \\
(\%)\end{array}$ & & $7.2 \%$ & $55.5 \%$ & $33.8 \%$ & $3.5 \%$ \\
\hline & $\begin{array}{c}\text { Worst case } \\
\text { scenario }\end{array}$ & & $13.2 \%$ & $8.8 \%$ & $-19.6 \%$ & $-2.4 \%$ \\
\hline & Location & Segment & $8.3 \%$ & $8.9 \%$ & $-15.2 \%$ & $-2.0 \%$ \\
\hline & AADT & $<10,000$ & $2.6 \%$ & $4.8 \%$ & $-6.4 \%$ & $-1.0 \%$ \\
\hline
\end{tabular}




\section{POLICY IMPLICATIONS}

The State of Oregon has adopted the Oregon Transportation Safety Action Plan to bring the number of fatal and severe crashes down to zero. The state and several cities across the world have committed to improve traffic safety, coordination between different stakeholders, and use of quantitative tools to make better decisions that will protect vulnerable users. These strategies will inform decision makers on prioritizing projects, programs and policies to fund, which will have the greatest benefit toward achieving the vision of zero fatalities and injuries (Oregon Department of Transportation, 2016).

This study is an important step toward informing risk factors that can be addressed to improve traffic safety in the State of Oregon. Findings of this research effort suggested that fatal and severe crashes were more common on roads that provide a good environment for high speed vehicles. We found evidence that variables such as arterials, wide roads, and high posted speed limits, increased the likelihood of fatal and severe crashes. When it comes to countermeasures related to infrastructure, working in road characteristics to reduce vehicle speed (i.e. narrow roads, local streets, low posted speed limits), have a potential to minimize fatalities and severe crashes.

Furthermore, the results of this research support policies and actions to separate vulnerable user transportation modes (walking and biking) from motorized modes. The literature suggests that vulnerable users prefer lower-stress roads to move, especially found when pedestrians and/or bicycles do not share the same space with drivers (Pucher, Dill, \& Handy, 2010). Separating heavy vehicles and high speed roads from vulnerable users can substantially bring down the number of fatal and severe crashes. Furthermore, providing separation for bicycles and pedestrians can help the state to promote more 
desirable environments for these transportation modes. Further work is needed to assess different separation alternatives that consider infrastructure cost.

Additionally, results of this study provided important insights to reduce crash severity for vulnerable users in state roads. Interventions and policies should focus in suburban and rural areas, targeting roads with geometric characteristics that allow high traffic speeds (i.e. wide roads with high number of lanes). Furthermore, educational programs and training classes should take place to teach drivers how to interact with vulnerable users. Special emphasis should be given to heavy vehicle drivers, which present the highest risk for both pedestrians and bicyclists.

Additionally, improving street lighting and making crosswalks and sidewalks more visible can have a positive impact on reduction of fatal and severe crashes. Furthermore, more strict controls and penalties should take place to reduce alcohol intoxicated drivers, especially when light conditions are poor.

This study also showed that elder pedestrians and bicyclists are at high risk if a crash occurs. Strategies to protect these users in recreational bike lanes and roads, and provision of accessible facilities and infrastructure can facilitate the movement of these users. Moreover, traffic engineers should design better traffic signals to accommodate the different walking speeds of senior pedestrians when crossing the road. 


\section{LIMITATIONS}

We advise the reader to be mindful of the focus and limitations of the study. This research concentrated on crashes that occurred on ODOT's facilities, which are mainly located in suburban and rural areas. Limiting crashes to state facilities reduced variability in some variables, such as posted speed limit, number of lanes, and road width (TABLE 33, TABLE 36, and TABLE 39).

A crash is included in the database if it involved a motorized vehicle, and if at least there was property damage over $\$ 1,500$. A crash that only involved two bicyclists, for example, is not included in the database. Additionally, a crash where a vehicle hit a pedestrian, but it did not cause an injury or property damage over the threshold, is not reported. These characteristics on how a crash is reported caused a bias on the data, resulting in more observations of fatal and severe injury crashes. This problem was evident for pedestrian crashes, where almost zero crashes were property only damage (severity level O). On top of this, researchers (Lord \& Mannering, 2010) have found that some crashes are not reported because the participants prefer to avoid the police or dealing with insurance issues.

We run the models dropping level O observations and we found the same risk factors and coefficients (by using the stepwise technique). The crash models were better to understand how risk factors increased the likelihood of going from level $\mathrm{B}$ or $\mathrm{C}$, to level K or A.

In terms of bicyclist crashes, there were more severity level O observations, likely to be product of the cost of the bicycle involved in the crash. When we dropped the 
severity level O observations, we found that AADT was not significant in the resulting model anymore.

Estimation of crash risk under various road characteristics is key to reduce the frequency of fatal and severe crashes. Nonetheless, assessing crash risk of vulnerable users has been a challenge among traffic safety researchers, since accurate information is not available. Since the probability of a crash occurring depends on measures of exposure, the lack of pedestrian and bicyclist volumes result in a loss of accuracy in estimating crash risks. We tried to control for exposure by using a crash risk ratio. This ratio considered VMT and percentage of pedestrian and bicyclist crashes (under several road conditions) as a measure of exposure; however, we acknowledge the importance of having data that can better reflect the actual exposure of vulnerable users. Furthermore, having vulnerable user exposure data can provide a better understanding of the relationship (if any) between crash frequency and crash severity.

This study also found that special pedestrian signals increased the likelihood of a crash being fatal. This finding can be related to the explanations highlighted in section 7.2; however, it may be also a problem with how this variable was reported in the database. The presence of a special pedestrian sign can be reported at locations where these treatments were not implemented, product of the lack of familiarity of people involved in the crash when reporting the crash. We explored the locations of the pedestrian crashes with special pedestrian signals reported and found that in most of them there was an RRFB or a median; however, there were some observations where there were also traffic signals. 
Furthermore, these special treatments are implemented in places where there are safety concerns (high crash frequency). The special pedestrian signs are useful to reduce number of crashes but may not fully solve severity issues when a crash occurs. For example (Monsere, Figliozzi, Kothuri, Razmpa, \& Hazel, 2016) found that crashes caused by no yielding were reduced at a significant level after RRFB installation; however, even if there was a reduction in fatal crashes, the change was small. Further research is needed to better understand this variable, for example, studies considering before and after special pedestrian sign implementation can be useful to quantify changes in severity levels over time.

Finally, we recommend for future research to assess how different bike facility designs (e.g. bike lane vs shared roadway) have an impact on crash severity. Our descriptive analysis showed that crashes in bike lanes tended to be level O (property only damage) rather than fatal or severe; however, the variable was not significant in the models. 


\section{CONCLUSION}

DOTs have an interest in increasing non-motorized transportation in the short and long term as a strategy to improve public health, air quality, and reduce traffic congestion. This interest has motivated researchers to explore what are the main strategies and policies that yield the maximum net benefit in terms of ridership and safety.

The goal of this study was to identify risk factors of crash severity for pedestrians and bicyclists in the Oregon Highway Network System, which is mainly located in suburban and rural areas. The findings of this observational study provide decision makers and planners the ability and information to improve safety conditions for both pedestrian and bicyclists in non- urban areas.

Risk factors such as age, vehicle type and movement, light conditions, road classification, traffic control device, posted speed limit, location of the pedestrian and wet road surfaces during clear weather conditions, were found to play an important role in the model for pedestrian crashes. For the bicyclist model, the findings suggested that age, crash location, vehicle movement and alcohol intoxication during dark conditions are statistically significant.

Models using only road and traffic characteristics were developed to provide more specific and tangible risk factors for DOTs to reduce fatal and severe crashes. Factors such as lack of streetlight at night, arterials, posted speed limit over $50 \mathrm{mph}$, high only truck volumes, and low traffic volumes (which result in higher speeds), were found to increase the likelihood of severe crashes.

Since most of the risk factors found in this study were associated with infrastructure that promotes high speed traffic, treatments to control how fast drivers are going are a feasible 
approach to protect vulnerable users. Similarly, policies and strategies to separate vulnerable users (walking and biking) from motorized modes are potential actions to reduce the fatal and severe crashes.

Targeting educational campaigns in rural areas may be key to preventing fatal and severe crashes related to vulnerable users. Training classes for heavy vehicle drivers in regards of pedestrians and bicyclists can help reduce crash severity as well. More strict policies and control should take place to reduce alcohol intoxicated drivers, especially when light conditions are poor. Moreover, more safe strategies should be implemented in recreational corridors and roads where seniors tend to walk or bike, since they were found to be the group with the highest risk if a crash occurs.

A direct application of this research is to map some of the risk factors to identify potential severity hot spots in the state network. This map can be included in future updates of the Oregon Bicycle and Pedestrian Plan. 


\section{REFERENCES}

1000 Friends of Oregon. (1993). Making the Land Use-Transportation-Air Quality Connection: Volume 4A, The Pedestrian Environment. Portland, OR.

A.Dijkstra, P.Marchesini, Bijleveld, F., V.Kars, H.Drolenga, \& Maarseveen, M. v. (2010). Do calculated conflicts in microsimulation model predict number of crashes? Transportation Research Record, 105-112.

Abdel-Aty, M., Lee, J., Siddiqui, C., \& Choi, K. (2013). Geographical unit based analysis in the context of transportation safety planning. Transportation Research Part A, $49,62-75$.

Abdel-Aty, M., Lee, J., Siddiqui, C., \& Choi, K. (2013). Geographical unit based analysis in the context of transportation safety planning. Transportatio Research Part A, $49,62-75$.

Abdul-Aziz, H., Ukkusuri, S., \& Hasan, S. (2013). Exploring the Determinants of Pedestrian-Vehicle Crash Severity in New York City. Accident Analysis and Prevention, 50, 1298-1309.

Accidents, T. R. (2009). Street Lighting and Road Safety. Edgbsaton, Birmingham.

Allen, B., Shin, T., \& Cooper, P. (1978). Analysis of traffic conflicts and collisions. Transportation Research Record(667), 67-74.

Allen-Munley, C., Daniel, J., \& Dhar, S. (2004). Logistic Model for Rating Urban Bicycle Route Safety. Transportation Research Record, 1878, 107-115.

Almodfer, R., Xiong, S., Fang, Z., Kong, X., \& Zheng, S. (2016). Quantitative analysis of lane-based pedestrian-vehicle conflict at a non-signalized marked crosswalk. Transportatio Research Part F, 42, 466-478. 
Almquist, S., Hyden, C., \& Risser, R. (1991). Use of speed limiters in cars for increased safety and a better environment. Transportation Rsearch Record, 1318, 34-39.

Al-Shammari, N., Bendak, S., \& Al-Gadhi, S. (2009). In-depth analysis of pedestrian crashes in Riyadh. Traffic Injury Prevention, 10(6), 556-559.

American Association of State Highway and Transportation Officials. (2010). Highway Safety Manual (Vol. 1st edition). Washington D.C.: AASHTO.

Amundsen, F., \& Hyden, C. (1977). Proceeding of First Workshop on Traffic Conflicts. Institute of Transport Economics, Lnd Institute of Technology.

Anderson, R., McLean, A., \& Farmer, M. (1997). Vehicle travel speeds and the incidence of fatal pedestrian crashes. Accident Analysis \& Prevention, 29(5), 667-674.

Archer, J. (2000). Developing the potential of micro-simulation modelling for traffic safety assessment. ICTCT. Corfu, Greece.

Archer, J. (2005). Methods for the assessment and prediction of traffic safety at urban intersection and their application in micro-simulation modeling. Royal Institute of Technology, Department of Infrastructure.

Ardeshiri, A., \& Jeihani, M. (2014). A speed limit compliance model for dynamic speed dispaly sign. Journal of Safety Research, 51, 33-40.

Arman, M. A., Rafe, A., \& Kretz, T. (2015). Pedestrian Gap Acceptance Behavior, A Case Study: Tehran. TRB 94th Annual Meeting Compendium of Pepers, 15-2217, pp. 1-15. Washington D.C.

Asadi-Shekari, Z., Mehdi, M., \& Muhammad Zaly, S. (2015). Pedestrian safety index for evaluating street facilities in urban areas. Safety Science, 74, 1-14. 
Asadi-Shekari, Z., Moeinaddini, M., \& Shah, M. Z. (2013). Non-motorised Level of Service: Addressing Challenges in Pedestrian and Bicycle Level of Service. Transport Reviews, 33(2), 166-194.

Asadi-Shekari, Z., Moeinaddini, M., \& Shah, M. Z. (2013). Non-motorised Level of Service: Addressing Challenges in Pedestrian and Bicycle Level of Service. Transport Reviews, 33(2), 166-194.

Autey, J., Sayed, T., \& Zaki, M. H. (2012). Safety evaluation of right-turn smart channels using automated traffic conflict analysis. Accident Analysis and Prevention, 45, 120-130.

Beck, B., Stevenson, M., Newstead, S., Cameron, P., Judson, R., Edwards, E., . . . Belinda, G. (2016). Bicycling crash characteristics: An in-depth crash investigation study. Accident Analysis and Prevention, 96, 219-227.

Bella, F., \& Silvestri, M. (2015). Effects of safety measures on driver's speed behavior at pedestrian crossings. Accident Analysis \& Prevention, 83, 111-124.

Benz, G. (1986). Pedestrian time-space concept, a new approach to the planning and design of pedestrian facili- ties. New York, NY: Parsons Brinckerhoff Quade and Douglas.

Beymer, D., McLauchlan, P., Coifman, B., \& Malik, J. (1997). A real-time computer vision system for measuring traffic parameters. Proceedings of the 1997 Conference on Computer Vision and Pattern Recognition (CVPR '97) (pp. 495501). Washingon D.C.: IEEE.

Bowman, B., \& Vecellio, R. (1994). Pedestrian Walking Speeds and Conflicts at Urban Media Locations. Transportation Research Record 1438, 67-73. 
Brewer, M., Fitzpatrik, K., J.A., J. W., \& Lord, D. (2006). Exploration of pedestrian gapacceptance behavior at selected locations. Transportation Research Record, Report No, 132-140.

Bullough, J., Rea, M., \& Zhou, Y. (2009). Analysis of Visual Performance Benefits from Roadway Lighting. NCHRP Project 5-19, Transportation Research Board of the National Academies, , Washington D.C.

Cafiso, S., García, A., Cavarra, R., \& Romero, M. A. (2011). Submitted to the 3rd International Conference on Road Safety and Simulation, (pp. 1-15). Indianapolis.

Caliendo, C., \& Guida, M. (2012). Microsimulation Approach for Predicting Crashes at Unsignalized Intersections Using Traffic Conflicts. Journal of Transportation Engineering, 1453-1467.

Carroll, P. S. (1973). Symposium on Driving Exposure. Ann Arbor, Michigan: Highway Safety Research Institute. The University of Michigan.

Charly, A. a. (2017). Estimation of Modified Time to Collision as Surrogate for MidBlock Crashes under Mixed Traffic Conditions. Transportation Research Board Annual Meeting. Washington, DC.

Chen, P.-L., Jou, R.-C., Saleh, W., \& Pai, C.-W. (2016). Accidents involving pedestrians with their backs to traffic or facing traffic: an evaluation of crash characteristics and injuries. Journal of Advanced Transportation, 50(5), 736-751.

Cheng, G., Wang, Y., \& Li, D. (2013). Setting conditions of crosswalk signal on urban road sections in China. 2013 International conference on transportation (pp. 96105). DEStech Publications. 
Cherry, C., Donlon, B., Yan, W., Moore, S., \& Xiong, J. (2012). Illegal mid-block pedestrian crossings in China: gap $\vee$ acceptance, conflict and crossing path analysis. International journal of injury control and safety promotion, 4, 320-330.

Chin, H., Quek, S., \& Cheu, R. (1991). Traffic conflicts in expressway merging. Journal of Transportation Engineering, 117(6), 663-643.

Chin, H.-C., \& Quek, S.-T. (1997). Measurement of Traffic Conflicts. Safety Science, 26(3), 169-185.

Christensen, R. (n.d.). Ordinal. Regression Models for Ordinal Data. Retrieved from http://www.cran.r-project.org/package=ordinal/

Clifton, K., \& Kreamer-Fults, K. (2007). An Examination of the Environmental Attributes Associated with Pedestrian Vehicle Crashes Near Public Schools. Accident Analysis and Prevention, 39, 708-712.

Cunto, F., \& Saccomanno, F. (2008). Calibration and validation of simulated vehicle performance at signalized intersections. Accident Analysis \& Prevention, 17, 1171-1179.

Currans, K., Gherke, S., \& Clifton, K. (2015). The Use of Images in Transportation Surveys: Testing Respondents Perceptions of Housing, Transportation and Built Environment Characteristics. Transportation Research Board 94th Annual Meeting, (pp. 1-20).

Denton, G. (1980). The influence of visual pattern on perceived speed. Perception 9, 4, 393-402. 
Dixon, L. (1996). Bicycle and Pedestrian level-of-service performance measures and standards for congestion management systems. Transportation Research Record, Report No 1538, 1-9.

El-Basyouny, K., \& Sayed, T. (2013). Safety performance functions using traffic conflicts. Safety Science, 51, 160-164.

Elliot \& Associates. (2005). Guidelines For Conducting A Focus Group.

Eluru, N., Bhat, C., \& Hensher, D. (2008). A mixed generalized ordered response model for examining pedestrian and bicyclist injury severity level in traffic crashes. Accident Analysis and Prevention, 40(3), 1033-1054.

Elvik, R. (1995). Meta-Analysis of Evaluations of Public Lighting as Accident Countermeasure. Transportation Research Record, Report No 1485, 112-123.

FDOT. (2009). Quality/Level of service Handbook. State of Florida Department of Transportaiton.

FHWA. (2002). Pedestrian Facilities User's Guide: Providing Safety and Mobility. FHWA-RD-01-102, U.S. Department of Transportation.

Figliozzi, M. A., \& Tipagornwong, C. (2016). Pedestrian Crosswalk Law: A study of traffic and trajectory factors that affect non-compliance and stopping distance. Accident Analysis and Prevention, 96, 169-179.

Figliozzi, M., Unnikrishnan, A., Kothuri, S., Caviedes, A., \& Soto, D. (2017). Compliance and surrogate safety measures for uncontrolled crosswalk crossings in Oregon: Tasks 2 and 3: Luterature review and crash data analysis report. Portland: Oregon Department of Transportation . 
Fitzpatrick, K., Turner, S., Brewer, M., Carlson, P., Ullman, B., Trout, N., . . Lord, D. (2006). Improving Pedestrian Safety at Unsignalized Crossings. Wasington, DC: Transit Cooperative Research Program and National Cooperative Highway Research Program of the Transportation Research Board.

Florida Department of Transportation. (2009). Quality/level of service. Tallahassee, FL.

Freitas, H., Oliveira, M., \& Jenkins, M. P. (1998). The focus group, a qualitative research method. Merrick School of Business, University of Baltimore, 1-22.

Fruin, J. (1971). Pedestrian planning and design. New York: Metropolitan Associations of Urban Designers and Environmental Planners.

Garder, P. (1989). Pedestrian Safety at Traffic Signals: A Study carried out with the help of a Traffic Conflicts Technique. Accident Analysis and Prevention, 435-444.

Garder, P. (2004). The impact of speed and other variables on pedestrian safety in Maine. Accident Analysis \& Prevention, 36, 533-542.

Gates, T. J., Savolainen, P. T., Stapleton, S., Kirsch, T., \& Miraskar, S. (2016). Development of Safety Performance Functions and Other Decision Support Tools to Assess Pedestrian and Bicycle Safety. Wayne State University. Transportation Research Center for Livable Communities (TRCLC).

Gettman, D., \& Head, L. (2003). Surrogate safety measures from traffic simulation models. Transportation Research Record, Paper No. 03-2958, 104-115.

Gettman, D., Pu, L., Sayed, T., \& Shelby, S. (2008). Surrogate safety assessment model and validation:Final report. Report No: FHWA-RD-03-050, Federal Highway Administration, US Department of Transportation, Washington D.C. 
Giahi Foormani, M., Alecsandru, C., \& McConnell, L. (2017). Safety Assessment of Backlit Pedestrian Crossing Sign on Stopping Characteristics at Unsignlized Intersection. Transportation Research Board Annual Meeting. Washington, DC: National Academy of Sciences.

GIZ. (2007). Gender and Urban Transport: Smart and Affordable. Federal Ministry for Economic Cooperation and Development.

Glauz, W., \& Migletz, D. (1980). Application of Traffic Conflicts Analysis at Intersections. Transportation Research Board.

Glennon, J., Glauz, W., M.C.Sharp, \& Thorson, B. (1977). Critique of the Traffic Conflict Techniques. Transportation Research Board, Report No. TRR 630, 32 38.

Glennon, J., Glauz, W., Sharp, M., \& Thorson, B. (1977). Critique of the Traffic Conflict Techniques. Transportation Research Board, Report No. TRR 630, 32-38.

Griffin, L., \& Reinhardt, R. (1996). A Review of Two innovative Pavement Patterns that have been Developed to Reduce Traffic Speed and Crashes. AAA Foundation for Traffic Safety, Washington D.C.

Guido, G., Saccomanno, F., A.Vitale, V.Astarita, \& D.Festa. (2011). Comparing Safety Performance Measures Obtained from Video Capture Data. Journal of Transportation Engineering, 137(7), 481-491.

Guo, F., Klauer, S. G., Hanley, J. M., \& Dingus, T. A. (2010). Near Crashes as Crash Surrogate for Naturalistic Driving Studies. Transportation Research Record, Report No. 2147, 66-74. 
Guo, Y., Liu, P., Liang, Q., \& Wang, W. (2016). Effects of parallelogram-shaped pavement markings on vehicle speed and safety of pedestrian crosswalks on urban roads in China. Accident Analysis \& Prevention, 95, 438-447.

Guozhu Cheng;Yuxia Wang;Dehuan Li. (2013). Setting conditions of crosswalk signal on urban road sections in China. 2013 International conference on transportation (pp. 96-105). DEStech Publications.

Guttinger, V. (1984). Conflict observation in theory and practice. (Springer-Verlag, Ed.) Berlin: International Study of Traffic Conflict Techniques.

Hagring, O. (2000). Effects of OD flows on roundabout entry capacity. Fourth International Symposium on Highway Capacity. Hawaii.

Haleem, K., Alluri, P., \& Gan, A. (2015). Analyzing pedestrian crash injury severity at signalized and non-signalized locations. Accident Analysis and Prevention, 81, $14-23$.

Haleem, K., Alluri, P., \& Gan, A. (2015). Analyzing pedestrian crash injury severity at signalized and non-signalized locations. Accident Analysis \& Prevention, 81, 1423.

Hansson, A. (1975). Studies in Driver Behaviour, with Applications in Traffic Design and Planning. Lund Institute of Technology, Department of Traffic Planning and Engineering. University of Lund.

Hauder, E., \& Garder, P. (1986). Research into the validity of traffic conflicts technique. Accident Analysis \& Prevention, 18, 471-481. 
Hauer, E. (1978, March 17). Design Consideration of Traffic Conflict Surveys. TRB Research Record 667 Highway Capacity, Measures of Effectiveness and Flow Theory, 57-66. Retrieved from ICTCT: ww.ictct.org/media/Hauer_1978a.pdf

Hauer, E. (1982). Traffic conflicts and exposure. Accident Analysis and Prevention, 359364.

Hauer, E. (1982). Traffic Conflicts and exposure. Accident Analysis \& Prevention, 14(5), $359-364$.

Hayward, J. (1972). Near miss determination through use of a scale of danger. Report No. TTSC 7115, Pennsylvania State University.

Henson, C. (2000). Levels of service for pedestrians. ITE journal, 70(9), 26-30.

Himanen, V., \& Kulmala, R. (1988). An application of logit models in analysing the behaviour of pedestrians and car drivers on pedestrian crossings. Accident Analysis \& Prevention, 20(3), 187-197.

Hine, J., \& Russell, J. (1993). Traffic barriers and pedestrian crossing behaviour. Journal of Transportation Geography, 1(4), 230-239.

Hipp, A., Manteiga, A., Burgess, A., Stylianou, A., \& Pless, R. (2016). Webcams, Crowdsourcing, and Enhanced Crosswalks: Developing a Novel Method to Analyze Active Transportation. Frontiers in Public Health, 4(97).

Horst, V. d. (1990). A time-based analysis of road user behavior in normal and critical encounter. Delft University of Technology.

Horst, V. d. (1991). Time-To-Collision as a cue for decision-making in braking. VIsion in Vehivles III, 19-26. 
Hunter, M., \& Rodgers, M. (2012). Evaluation of Intersection Countermeasures on HighSpeed Rural Multi-lane Facilities. US Department of Transportation, Federal Highway Administration. Atlanta: Georgia Tech Research Corporation.

Hydén, C. (1977). A Traffic Conflict Technique for Examining Urban Intersection Problems. First Workshop on Traffic Conflicts (pp. 87-98). Oslo, Norway: Royal Norwegian Council for Industrial and Scientific Research.

Hydén, C. (1987). The development of a method for traffic safety evaluation: The Swedish Traffic Conflicts Technique. Bulletin 70, Lund University.

Hydén, C., \& Várhelyi, A. (2000). The effects of safety, time consumption and environment of large scale use of roundabouts in an urban area: A case study. Accident Analysis \& Prevention 32, 11-23.

InDev. (2016). Review of Current Study Methods for Vulnerable Road Users Safety. Warsaw, Poland: Horizon 2020.

Islam, S., \& Hossain, A. (2015). Comparative Analsyis of Injury Severity Resulting from Pedestrian-Motor Vehicle and BIcycle-Motor Vehicle Crashes on Roadways in Alabama. Transportation Research Record, 2514, 79-87.

Ismail, K., Sayed, T., \& Saunier, N. (2011). Methodologies for aggregating traffic conflict indicators. Transportation Research Record, Record No 2237, 10-19.

Ismail, K., Sayed, T., Saunier, N., \& Lim, C. (2010). Automated analysis of pedestrianvehicle conflicts using video data. Transportation Research Recodr: Journal of the Transportation Research Board, 2140, 44-54.

JAMAR Technologies, Inc. (2016). Products. Retrieved 12 14, 2016, from http://www.jamartech.com/index.html\#services 
Jang, K., Park, S., Kang, S., Song, K., Kang, S., \& Chung, S. (2013). Evaluation of pedestrian safety: geographical identification of pedestrian crash hotspots and evaluating risk factors for injury severity. Proceedings of the 92nd Annual Meeting of the Transportation Research Board. Washington, D.C.

Kash, G. (2013). Open Versus Closed Effects of Question Form on Transit rider Expressions of Policy Preferences in arequipa, Peru. Transportation Research Record: Journal of the Transportation Research Board, 2354, 51-58.

Kash, G. (2014). Gendered Perspectives on Transit Crime in Arequipa, Perú and Bogotá, Colombia. Presented Transportation Research Board 2014 Annual Meeting, 1-16.

Kashani, A. T., \& Besharati, M. M. (2016). Fatality rate of pedestrians and fatal crash involvement rate of drivers in pedestrian crashes: a case study of Iran. International Journal of Injury Control and Safety Promotion, 1-10.

Katz, A., Zaidel, D., \& Elgrishi, A. (1975). An experimental study of driver and pedestrian interaction during the crossing conflict. Journal of the Human Factors and Ergonomics Society, 17, 514-527.

Khisty, C. (1994). Evaluation of pedestrian facilities. Beyond the level-of-service concept. Transportation Research Record, Report No 1438, 45-50.

Kim, J., G.F., U., Shankar, V., \& Kim, S. (2008). Age and pedestrian injury severity in motorvehicle crashes: a heteroskedastic logit analysis. Accident and Prevention, 40(5), 1695-1702.

Kim, J., Kim, S., G., U., \& Porello, L. (2007). BIcyclist Injury Severities in BicycleMotor-Vehicle Accidents. Accident Analysis and Prevention, 39, 238-251. 
Kim, K., Brunner, I., \& Yamashita, E. (2006). Influence of land use, population, employment, and economic activity on accidents. Transportation Research Record, Report No 1953, 56-64.

Kim, K., Brunner, I., \& Yamashita, E. (2008). Modeling violation of Hawaii's crosswalk law. Accident Analysis \& Prevention, 40(3), 894-904.

Kim, K., Nitz, L., Richardson, J., \& Li, L. (1995). Personal and Behavioral Predictors of Automobile Crash and Injury Severity. Accident Analysis \& Prevention, 24(4), $469-481$.

Kittelson \& Associates, Inc. (2014). Pedestrian and Bicycle Safety Implementation Plan. Oregon Department of Transportation, Bend.

Kitzinger, J. (1995). Qualitative Research: Introducing focus groups. BMJ, 311-299.

Klauer, S., Dingus, T., Neale, V., Sudweeks, J., \& Ramsey, D. (2006). The Impact of Driver Inattention on Near-Crash/Crash Risk: An Analysis Using 100-Car Naturalistic Driving Study Data. Virginia Tech Transportation Institute. National Highway Traffic Safety Administration.

Krueger, R. (2002). Designing and Conducting Focus Group Interviews. University of Minnesota, St. Paul.

Kruysee, H. (1991). The Subjective Evaluation of Traffic Conflicts Based on an Internal Concept of Dangerousness. Accident Analysis \& Prevention, 23(1).

Landis, B., Vattikuti, V., Ottenberg, R., McLeod, D., \& Guttenplan, M. (2001). Modeling the roadside walking environment: A pedestrian level of service. Transportation Research Record, Report No 1773, 82-88. 
Laureshyn, A., A.Svensson, \& C.Hydem. (2010). Evaluation of traffic safety, based on micro-level behavioral data: theoretical framework and first implementation. Accident Analysis \& Prevention, 42(6), 1636-1646.

Lee, C., \& Abdel-Aty, M. (2003). Yokohama, Japan. Lee C, Abdel-Aty M. 2005. Comprehensive analysis of vehicle-pedestrian crashes at intersections in Florida. Accident Analysis and Prevention, 37, 775-786.

Lee, J., Abdel-Aty, M., \& Jiang, X. (2015). Multivariate crash modeling for motor vehicle and non-motorized modes at the macroscopic level. Accident Analysis \& Prevention, 78, 146-154.

Li, D., Ranjitkar, P., Zhao, Y., Yi, H., \& Rashidi, S. (2016). Analyzing pedestrian crash injury severity under different weather conditions. Traffic Injury Prevention, $18(4), 427-430$.

Litman, T. (2017). Evaluating Transportation Equity: Guidance for Incorporating Distributional Impacts in Transportation Planning. Victoria Transport Policy Institute.

Liu, P., Zhang, X., Wang, W., \& Xu, C. (2011). Driver response to automated speed enforcement on rural highways in China. Transportation Research Record, Report No 2265, 109-117.

Liu, Y., \& Tung, Y. (2014). Risk analysis of pedestrians' road-crossing decisions: Effects of age, time gap, time of the day, and vehicle speed. Safety Science, 63, 77-82.

Lord, D., \& Mannering, F. (2010). The statistical analysis of crash-frequency data: A review and assessment of methodological alternatives. Transportation Research Part A: Policy and Practice, 44(5), 291-305. 
Loukatiou-Sideris, A., Liggett, R., \& Sung, H. (2007). Death on the crosswalk: a study of pedestrian-automobile collisions in Los Angeles. Journal of Planning Education and Research, 26(3), 338-351.

M.Buhrmester, Kwang, T., \& Gosling, S. (2011). Amazon's Mechanical Turk: a new source of inexpensive, yet high-quality, data? Perspectives on Psychological Sciences, 6(1), 3-5.

Malaterre, G., \& Muhlrad, H. (1977). A conflict technique. First Workshop on Traffic Conflicts., (pp. 47-58). Oslo.

Malkhamah, S., Tight, M., \& Montgomery, F. (2005). The development of an automatic method of safety monitoring at Pelican crossings. Accident Analysis \& Prevention, 37, 938-946.

Mason, J. (2002). Qualitative Researching. London: SAGE.

Matsui, Y., \& Oikawa, S. (2016). Association of Impact Velocity with Risks of Serious Injuries and Fatalities to Pedestrians in Commercial Truck-Pedestrian Accidents. Stapp Car Crash Journal, 60, 165-182.

McIntyre, A. (2016). Determinants of Bicycle and Pedestrian Crash Severity in San Francisco, CA. Arizona State University: A Thesis Presented in Partial Fulfillment of the Requirements for the Degree Master of Science.

Mead, J., Zegeer, C., \& Bushell, M. (2014). Evaluation of Pedestrian-Related Roadway Measures: A Summary of Available Research. (U. H. Center, Ed.) Pedestrian and Bicycle Information Center. 
Miller, J. S., Bigelow, J. A., \& Garber, N. J. (2000). Calibrating Pedestrian Level-ofService Metrics with 3-D Visualization. Transportation Research Record, Report No $1705,9-15$.

Minderhound, M., \& Bovy, P. (2001). Extended time-to-collission measures for traffic safety assessment. Accident Analysis \& Prevention, 33(1), 89-97.

Monsere, C., Figliozzi, M., Kothuri, S., Razmpa, A., \& Hazel, D. (2016). Safety effectiveness of pedestrian crossing enhancements. Salem: Oregon Department of Transprotation.

Mozer, D. (1994). Calculating Multi-Mode Levels-of-Service. International Bicycle Fund.

Namgung, M., \& Akar, G. (2014). Role of Gender and Attitudes on Public Transportation Use. Transportation Research Record: Journal of the Transportation Research Board, 2415, 136-144.

Nassar, J., \& Troyer, D. (2013). Pedestrian injuries due to mobile phone use in public places. Accident Analysis \& Prevention, 57, 91-95.

NCHRP. (2008). Multimodal Level of Service Analysis for Urban Streets. Washington D.C.: Transportation Research Board.

Newcomer, K., Hatry, H., \& Wholey, J. (2015). Handbook of Practical Program Evaluation: Edition 4. In Chapter 20: Focus Group Interviewing (Vol. Chapter 20, pp. 506-534). Jossey-Bass.

Niaki, M. S., Fu, T., Saunier, N., Miranda-Moreno, L. F., Amador, L., \& Bruneau, J.-F. (2016). Road Lighting Effects on Bicycle and Pedestrian Accident Frequency 
Case Study in Montreal, Quebec, Canada. Transportation Research Record, Report No 2555, 86-94.

Nilsson, G. (1978). Risk Exposure. A study of needs of risk exposure for road accident analysis. VTI Rapport.

Nordback, K., Kothuri, S., Petritsch, T., McLeod, P., Rose, E., \& Twaddell, H. (2016). Exploring Pedestrian Counting Procedures. Washington, DC: Federal Highway Administration.

O'Flaherty, C., \& Parkinson, M. (1972). Movement in a city centre footway. Traffic Engineering and Control, 13, 434-438.

Oh, C., Kang, Y., Kim, B., \& Kim, W. (2005). Analysis of pedestrian-vehicle crashes in Korea: focused on developing probabilistic pedestrian fatality model. Proceedings of the 84th Annual Meeting of the Transportation Research Board. Washington, D.C.

Ole, P. (2009). Effects of road lighting: An analysis based on Dutch accident statistics 1987-2006. Accident Analysis and Prevention 41.

Oregon Department of Transportation. (2014). ODOT Statewide Crash Data System: Motor Vehicle Traffic Crash Analysis and Code Manual. Oregon: Trasnportation Data Section Crash ANalysis and Reporting Unit.

Oregon Department of Transportation. (2016). Oregon Bicycle and Pedestrian Plan. Salem, OR.

Oregon Department of Transportation. (2016). Oregon Transportation Safety Action Plan. Salem. 
Oxley, J., O'Hern, S., Burtt, D., \& Rossiter, B. (2017). Falling while walking: A hidden contributor to pedestrian injury. Accident Analysis and Prevention, 1-6.

Ozbay, K., Yang, H., Bartin, B., \& Mudigonda, S. (2008). Derivation and Validation of a New Simulation-Based Surrogate Safety Measure. Transportation Research Record 2083, 105-113.

Perkin, S., \& Harris, J. (1967). Criteria for Traffic Conflict Characteristics: Signalized Intersections. General Motors Corporation, Electro-Mechanical Department.

Petritsch, T. A., Landis, B. W., McLeod, P. S., Huang, H. F., Challa, S., Skaggs, C. L., . . . Vattikuti, V. (2006). Pedestrian Level-of-Service Model for Urban Arterial Facilities with Sidewalks. Transportation Research Record, Report No 1982, 8489.

Pirdavani, A., Brijis, T., T.Bellemans, \& G.Wets. (2010). Evaluation of traffic safety at un-signalized intersections using microsimulation:A utilization of proximal safety indicators with TSC-Sim. Accident Analysis \& Prevention, 26, 593-607.

Pucher, J., Dill, J., \& Handy, S. (2010). Infrastructure, programs, and policies to increase bicycling: An international review. Preventive Medicine, 50, S106-S125.

Rea, M., Bullough, J., \& Zhou, Y. (2010). A method for assessing the visibility benefits of roadway lighting. Lighting Research and Technology, 42(2), 215-241.

Ripley, B., Venables, B., Bates, D., Hornik, K., Gebhardt, A., \& Firth, D. (n.d.). MASS. Modern Applied Statistics with S (4th edition). Retrieved from http://www.stats.ox.ac.uk/pub/MASS4/

Sarkar, S. (1993). Determination of service levels for pedestrians, with European example. Transportation Research Record, Report No 1405, 35-42. 
Sarkar, S. (2002). Qualitative evaluation of comfort needs in urban walkways in major activity centers. Transportation Research Board Conference, (pp. 13-17). Washington D.C.

Sarkar, S., Richard, T., \& Hunt, J. (2011). Logistic regression model of risk of fatality in vehicle-pedestrian crashes on national highways in Bangladesh. Transportation Research Record, Report No 2264, 128-137.

Sasidharan, L., Wu, K.-F., \& Menendez, M. (2015). Exploring the application of latent class cluster analysis for investigating pedestrian crash injury severities in Switzerland. Accident Analysis and Prevention, 85, 219-228.

Saunier, N., \& Sayed, T. (2006). A feature-based tracking algorith for vehicles in intersections. Proceedings of the 3rd Canadian Conference on COmputer and Robot Vision (CRV'06). IEEE.

Savolainen, P., Mannering, F., Lord, D., \& Quddus, M. (2011). The statistical analysis of highway crash-injury severities: A review and assessment of methodological alternatives. Accident Analysis and Prevention, 1666-1676.

Sayed, \& Zein. (1999). Traffic Conflict standards for intersections. Transportation Planning and Technology, 22, 309-323.

Schroeder, B., \& Rouphail, N. (2011). Event-based modeling of driver yielding behavior at unsignalized crosswalks. Journal of Transportation Engineering, 137, 455-465.

Sheng, J., Wang, D.-h., Xu, C., \& Ma, D.-f. (2013, April). Short-term traffic safety forecasting using Gaussian mixture model and Kalman filter. Journal of Zhejiang University SCIENCE A, 14(4), 231-243. 
Shoarian-Sattari, K., \& Powell, D. (1987). Measured Vehicle Flow Parameters as Predictors in Road Traffic Accident Studies. Traffic Engineering and Control, $28(6), 328-335$.

Shurbutt, J., Houten, R. V., Turner, S., \& Huitema, B. (2009). Analysis of effects of LED rectangular rapid-flash beacons on yielding to pedestrians in multilane crsswalks. Transportation Research Record, Report No 2140, 85-95.

Singh, K., \& Jain, P. (2011). Methods of assessing pedestrian level of service. Journal of Engineering Research and Studies, 2, 116-124.

Small, M. L. (2011). How to Conduct a Mixed Methods Study: Recent Trents in a Rapidly Growing Literature. The Annual Review of Sociology .

Smartmicro. (2016). Smart Microwave Sensors. Retrieved 12 14, 2016, from Smartmicro traffic Radars: http://www.smartmicro.de/traffic-radar/traffic-radar-overview/

Songchitruska, P., \& Tarko, A. P. (2004). Using Imaging Technology to Evaluate Highway Safety. Purdue University, Joint Transportation Research Program. Indiana Department of Transportation and Federal Highwat Administration.

Stipanic, J., \& Miranda-Moreno, L. (2015). Traffic Parameter Methods for Surrogate Safety: A Comparative Study of Three Mobile Sensor Technologies. TRB Annual Meeting. Washington, DC: Transportation Research Board.

Strauss, J., Miranda-Moreno, L. F., \& Morency, P. (2014). Multimodal injury risk analysis of road users at signalized and non-signalized intersections. Accident Analysis \& Prevention, 71, 201-209. 
Strauss, J., Miranda-Moreno, L. F., \& Morency, P. (2014). Multimodeal injury risk analysis of road users at signalized and non-signalized intersections. Accident Analysis \& Prevention, 71, 201-209.

Svensson, A. (1998). A Method For Analyzing the Traffic Process In A Safety Perspective. Department of Traffic Planning and Engineering, Lund University, Lund, Sweeden.

Sze, N., \& Wong, S. (2007). Diagnostic analysis of the logistic model for pedestrian injury severity in traffic crashes. Accident Analysis and Prevention, 39(6), 12671278.

Tarko, A. P. (2012). Use of crash surrogates and exceedance statistics to estimate road safety. Accident Analysis \& Prevention, 45, 230-240.

Tarko, A. P., Davis, G., Saunier, N., Sayed, T., \& Washington, S. (2009). Surrogate Measures of Safety. ANB20 Comittee on Safety Data Evaluation and Analysis, ANB20(3) Subcomittee on Surrogate Measures of Safety.

Tarko, A., \& Azam, M. (2011). Pedestrian injury analysis with consideration of the selectivity bias in linked police-hospital data. Accident and Prevention, 43, 16891695.

Tay, R., Choi, J., Kattan, L., \& Khan, A. (2011). A Multinomial Logit Model of Pedestrian-Vehicle Crash Severity. International Journal of Sustainable Transportation, 5, 233-249.

Thambiah, M., \& Toru, H. (2007). Overall Level of Service of Urban Walking Environment and Its Influence onPedestrian Route Choice Behavior: Analysis of 
Pedestrian Travel in Sapporo, Japan. Transportation Research Record, Report No 2002, 7-17.

Tiwari, G., D.Mohan, \& J.Fazio. (1999). Conflict Analysis for Prediction of Fatal Crash Locations in Mixed Traffic Steams. Accident Analysis \& Prevention, 30(2), 207215.

Tiwari, G., Mohan, D., \& Fazio, J. (1998). Conflict analysis for prediction of fatal crash locations in mixed traffic streams. Accident Analysis and Prevention, 30(2), 207215.

Tom, A., \& Granié, M.-A. (2011). Gender differences in pedestrian rule compliance and visual search at signalized and unsignalized crossroads. Accident Analysis and Prevention, 43, 1794-1801.

Torbic, D., Hutton, J., Bokenkroger, C., Bauer, K., Donnell, E., Lyon, C., \& Persaud, B. (2010). Guidance on design and application of rumble strips. Transportation Research Record, 2149(1), 59-69.

U.S. Department of Transportation. (2015). Safer People Safer Streets: Pedestrian and Bicycle Safety Initiative.

Ukkusuri, S., Hasan, S., \& Aziz, H. (2011). Random parameter model used to explain effects of built-environment characteristics on pedestrian crash frequency. Transportation Research Record, Report No 2237, 98-106.

Várhely, A. (1998). Drivers' speed behaviour at a zebra crossing: a case study. Accident Analysis \% Prevention, 30(6), 731-743. 
Várhelyi, A. (1996). Dynamic Speed Adaptation Based on Information Technology-A Theoretical Background. Bulletin 142, Lund University, Department of Traffic Planning and Engineering.

Várhelyi, A. (1998). Drivers' speed behaviour at a zebra crossing: a case study. Accident Analysis \& Prevention, 30(6), 731-743.

Vogel, K. (2002). What characterizes a "free vehicle" in an urban area? Transportation Research F, 5, 313-327.

Vogel, K. (2003). A comparison of headway and time to collision as safety indicators. Accident Analysis \& Prevention, 35, 427-473.

Walz, F., Hoefliger, M., \& Fehiman, W. (1983). Speed limit reduction from 60 to 50 $\mathrm{km} / \mathrm{h}$ and pedestrian injuries. Proceedings of the 27th Stapp Car Crash Conference with International Research Commitee on Biokinetics of Impact, (p. $277=285)$.

Wang, X., Yang, J., Lee, C., Ji, Z., \& You, S. (2016). Macro-level safety analysis of pedestrian crashes in Shanghai, China. Accident Analysis \& Prevention, 96, 1221.

Wanvik, P. (2009). Road Lighting and Traffic Safety: Do we Need Road Lighting? PhD dissertation, Norweigan University of Science and Technology, Trondheim.

Washington, S., Haque, M. M., Oh, J., \& Lee, D. (2014). Applying quantile regression for modeling equivalent property damage only crashes to identify accident blackspots. Accident Analysis and Prevention, 66, 136-146. 
Wazana, A., Rynard, V., \& Raina, P. (2000). Are child pedestrians at increased risk of injury on one-way compared to two-way streets? Canadian Journal of Public Health, 91, 201-206.

Weiss, R. (1994). Learning from strangers. New York: The free press.

Wier, M., Weintraub, J., Humphreys, E., Seto, E., \& Bhatia, R. (2009). An area-level model of vehicle-pedestrian injury collisions with implications for land use and transportation planning. Accident Analysis \& Prevention, 41, 137-145.

Williams, M. (1981). Validity of the Traffic conflicts technique. Accident Analysis and Prevention, 13, 133-145.

Wisel, V. (2017). Focus Groups. Portland State University, Portland, OR.

Wu, K., \& Jovanis, P. (2012). Crashes and crash-surrogate events: Exploratory modeling with naturalistic driving data. Accident Analysis \& Prevention, 45, 507-516.

Yagil, D. (2000). Beliefs, motives and situational factors related to pedestrians' selfreorted behavior at signal-controlled crossings. Transportation Research Part F: Traffic Psychology and Behaviour, 3(1), 1-13.

Yamamoto, T., Hashiji, J., \& Shankar, V. (2008). Underreporting in traffic accident data, bias in parameters and the structure of injury severity models. Accident Analysis and Prevention, 40, 1320-1329.

Yannis, G., Papadimitriou, E., \& Theofilatos, A. (2013). Pedestrian gap acceptance for mid-block street crossing. Transportation Planning and Technology, 36(5), 450462.

Zahabi, S. A., Strauss, J. M., \& Miranda-Moreno, L. F. (2011). Estimating Potential Effect of Speed Limits, Build Environment, and Other Factors on Severity of 
Pedestrian and Cyclist injuries in crashes. Transportation Research Record, 2247, 81-60.

Zajac, S., \& Ivan, J. (2003). Factors influencing injury severity of motor vehicle-crossing pedestrian crashes in rural Connecticut. Accident Analysis \& Prevention, 35, 369379.

Zajíc, P. (2012). Traffic conflicts and road transport safety - New development. Electronic Technical Journal of Technology, Engineering and Logistics in Transport, 7(4), 174-183.

Zeeger, C., Stewart, J., Huang, H., \& Lagerway, P. (2001). Safety effects of marked versus unmarked crosswals at uncontrolled locations: analysis of pedestroan crashes in 30 cities. Transportation Research Record, Report No 1773, 56-68.

Zegeer, C. V., Carter, D. L., Hunter, W. W., Stewart, J. R., Huanf, H., Do, A., \& Sandt, L. (2006). Index for Assessing Pedestrian Safety at Intersections. Transportation Research Record, Report No 1982, 76-83.

Zegeer, C., Stewart, J., Huang, H., \& Lagerway, P. (2001). Safety effects of marked versus unmarked crosswals at uncontrolled locations: analysis of pedestrIan crashes in 30 cities. Transportation Research Record, Report No 1773, 56-68.

Zegeer, C., Stewart, J., Huang, H., \& Lagerway, P. (2001). Safety effects of marked versus unmarked crosswals at uncontrolled locations: analysis of pedestroan crashes in 30 cities. Transportation Research Record, Report No 1773, 56-68.

Zhang, G., Yau, K., \& Zhang, X. (2014). Analyzing fault and severity in pedestrianmotor vehicle accidents in China. Accident Analysis and Prevention, 73, 141-150. 
Zhang, L., Ismail, K., \& Meng, X. (2014). Traffic conflict techniques for road safety analysis: Open questions and some insiights. Canadian Journal of Civil Engineering, 41(7), 633-641.

Zhao, H., Yin, Z. Y., Che, X., Xie, J., Hiang, W., \& Wang, Z. (2014). Analysis of 121 fatal passenger car-adult pedestrian accidents in China. Journal of Forensic and Legal Medicine, 27, 76-81.

Zhao, Y., Bai, Y., \& Yang, X. (2011). Modeling Conflicts between Right-turning Vehicles and Pedestrians at Signalized Intersections. International Conference on Transportation Information and Safety (pp. 529-536). Wuhan, China: Transportation and Development Institute of the American Society of Civil Engineers.

Zheng, Y. (2014 ). Analysis of Factors Influencing Pedestrian Injury Severity in Pedestrian-Vehicle Crashes. T\&DI Congress (pp. 448-457). ASCE.

Zhuang, X., \& Wu, C. (2011). Pedestrians' crossing behaviors and safety at unmarked roadway in China. Accident Analysis \& Prevention, 43(6), 1927-1936. 


\section{APPENDICES}

\section{Appendix A: risk ratio by neighborhood concept}

Crash data from the Oregon Statewide Crash Data System was grouped by intersection and segment based on the traffic control device information available at the crash level. If a crash occurred at any type of intersection (controlled and uncontrolled), it was grouped in the uncontrolled category. If the crash was reported in a segment, then it was grouped in the segment category. Crashes at driveways, curves, bridges structures were not taken into account due to the few observations available. Crash information is presented as the number of crashes reported. Furthermore, crashes were grouped by neighborhood concept (Currans, Gherke, \& Clifton, 2015) to account for the differences in built environment in the likelihood of a crash occurrence. 


\section{A. Pedestrian exposure analysis by neighborhood concept}

TABLE 61: Pedestrian Risk ratio. Posted Speed limit by neighborhood concept D

\begin{tabular}{l|l|l|l|l|l|l|l|l|l|l|l}
\hline \hline & \multicolumn{9}{|c}{ INTERSECTION } & \multicolumn{1}{c}{ SEGMENT } \\
\hline POSTED SPEED LIMIT & $\mathbf{K}$ & $\mathbf{A}$ & $\mathbf{B}$ & $\mathbf{C}$ & $\mathbf{O}$ & $\mathbf{K}$ & $\mathbf{A}$ & $\mathbf{B}$ & $\mathbf{C}$ & $\mathbf{O}$ \\
\hline$<20$ & NA & NA & NA & NA & NA & NA & NA & NA & NA & NA \\
\hline $20-35$ & 4.29 & 4.51 & 4.46 & 4.62 & 7.73 & NA & 1.25 & 2.76 & 3.73 & NA \\
\hline $35-50$ & 2.26 & 2.31 & 2.15 & 2.31 & 0.79 & 3.30 & 3.32 & 2.76 & 2.60 & NA \\
\hline $50-65$ & 0.15 & 0.10 & 0.17 & 0.09 & 0.31 & 0.17 & 0.10 & 0.11 & 0.08 & NA \\
\hline$>65$ & NA & NA & NA & NA & NA & 2.60 & $*$ & NA & NA & NA & NA \\
\hline \hline
\end{tabular}

TABLE 62: Pedestrian Risk ratio. Posted Speed limit by neighborhood concept E

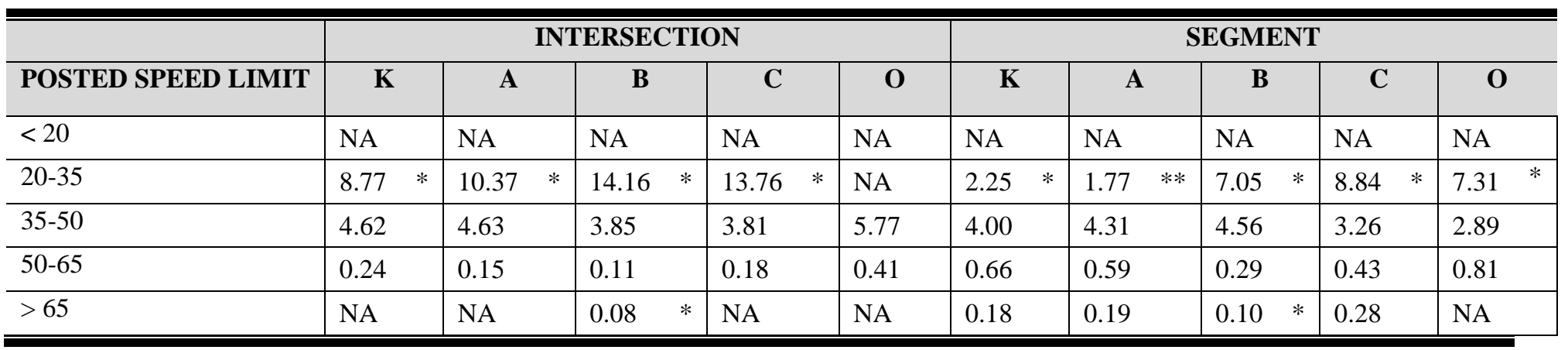


TABLE 63: Pedestrian Risk ratio. Posted Speed limit by neighborhood concept F

\begin{tabular}{|c|c|c|c|c|c|c|c|c|c|c|}
\hline \multirow[b]{2}{*}{ POSTED SPEED LIMIT } & \multicolumn{5}{|c|}{ INTERSECTION } & \multicolumn{5}{|c|}{ SEGMENT } \\
\hline & $\mathbf{K}$ & $\mathbf{A}$ & B & $\mathbf{C}$ & $\mathbf{O}$ & $\mathbf{K}$ & $\mathbf{A}$ & $\mathbf{B}$ & $\mathbf{C}$ & $\mathbf{O}$ \\
\hline$<20$ & NA & NA & NA & NA & NA & NA & NA & NA & NA & NA \\
\hline $20-35$ & NA & NA & NA & NA & NA & NA & NA & NA & NA & NA \\
\hline $35-50$ & 8.18 & 6.55 & 8.66 & 6.55 & 13.09 & 3.98 & 6.86 & 4.36 & 5.82 & NA \\
\hline $50-65$ & 0.50 & 0.71 & 0.18 & 0.27 & NA & 1.29 & 0.76 & 0.91 & 0.77 & NA \\
\hline$>65$ & 0.30 & NA & NA & $0.06 *$ & NA & $0.11 *$ & $0.12 *$ & 0.20 & 0.14 & NA \\
\hline
\end{tabular}

TABLE 64: Pedestrian Risk ratio. Road width by neighborhood concept D

\begin{tabular}{|c|c|c|c|c|c|c|c|c|c|c|}
\hline \multirow[b]{2}{*}{$\begin{array}{l}\text { WIDTH } \\
\text { (ft.) }\end{array}$} & \multicolumn{5}{|c|}{ INTERSECTION } & \multicolumn{5}{|c|}{ SEGMENT } \\
\hline & $\mathbf{K}$ & $\mathbf{A}$ & B & $\mathrm{C}$ & $\mathbf{O}$ & $\mathbf{K}$ & $\mathbf{A}$ & B & $\mathrm{C}$ & $\mathbf{O}$ \\
\hline $0-10$ & NA & NA & NA & NA & NA & NA & NA & NA & NA & NA \\
\hline $10-20$ & NA & NA & $0.30 *$ & $0.21 *$ & NA & NA & NA & NA & NA & NA \\
\hline $20-30$ & 0.94 & 0.55 & 0.83 & 0.83 & 1.98 & 0.55 & 0.32 & 0.53 & 0.26 & NA \\
\hline $30-40$ & 0.25 & 0.35 & 0.41 & 0.47 & 0.53 & 0.44 & 0.34 & 0.33 & 0.14 & NA \\
\hline $40-50$ & 2.52 & 2.85 & 2.49 & 2.18 & 1.18 & 2.29 & 3.23 & 3.26 & 3.87 & NA \\
\hline $50-60$ & $7.75 *$ & $8.14 *$ & $4.35 *$ & $4.31 *$ & NA & $11.30 \quad *$ & $7.88 \quad *$ & $4.36 *$ & $7.50 *$ & NA \\
\hline $60-70$ & NA & NA & NA & NA & NA & NA & NA & NA & NA & NA \\
\hline$>80$ & NA & NA & NA & NA & NA & NA & NA & NA & NA & NA \\
\hline
\end{tabular}


TABLE 65: Pedestrian Risk ratio. Road width by neighborhood concept E

\begin{tabular}{|c|c|c|c|c|c|c|c|c|c|c|}
\hline \multirow[b]{2}{*}{$\begin{array}{l}\text { WIDTH } \\
\text { (ft.) }\end{array}$} & \multicolumn{5}{|c|}{ INTERSECTION } & \multicolumn{5}{|c|}{ SEGMENT } \\
\hline & $\mathbf{K}$ & $\mathbf{A}$ & B & $\mathbf{C}$ & $\mathbf{O}$ & $\mathbf{K}$ & $\mathbf{A}$ & B & $\mathrm{C}$ & $\mathbf{O}$ \\
\hline $0-10$ & NA & NA & NA & NA & NA & NA & NA & NA & NA & NA \\
\hline $10-20$ & NA & NA & $0.40 *$ & $0.43 *$ & NA & NA & NA & NA & NA & NA \\
\hline $20-30$ & 0.43 & 0.43 & 0.39 & 0.41 & 0.71 & 0.77 & 0.66 & 0.74 & 0.81 & NA \\
\hline $30-40$ & 0.87 & 0.49 & 0.69 & 0.83 & NA & 0.42 & $0.26 \quad *$ & 0.43 & 0.79 & 2.18 \\
\hline $40-50$ & 2.85 & 3.29 & 2.89 & 2.95 & 2.59 & 2.89 & 3.14 & 2.33 & 2.09 & 1.30 \\
\hline $50-60$ & NA & NA & NA & NA & NA & NA & NA & NA & NA & NA \\
\hline $60-70$ & NA & NA & NA & NA & NA & NA & NA & NA & NA & NA \\
\hline$>80$ & NA & NA & NA & NA & NA & NA & NA & NA & NA & NA \\
\hline
\end{tabular}

TABLE 66: Pedestrian Risk ratio. Road width by neighborhood concept F

\begin{tabular}{|c|c|c|c|c|c|c|c|c|c|c|}
\hline \multirow[b]{2}{*}{$\begin{array}{l}\text { WIDTH } \\
\text { (ft.) }\end{array}$} & \multicolumn{5}{|c|}{ INTERSECTION } & \multicolumn{5}{|c|}{ SEGMENT } \\
\hline & $\mathbf{K}$ & $\mathbf{A}$ & B & $\mathbf{C}$ & $\mathbf{O}$ & $\mathbf{K}$ & $\mathbf{A}$ & B & $\mathbf{C}$ & $\mathbf{O}$ \\
\hline $0-10$ & NA & NA & NA & NA & $\mathrm{NA}$ & $\mathrm{NA}$ & $\mathrm{NA}$ & $\mathrm{NA}$ & $\mathrm{NA}$ & NA \\
\hline $10-20$ & NA & NA & NA & 1.25 & NA & NA & NA & NA & NA & NA \\
\hline $20-30$ & 0.84 & 0.60 & 0.47 & 0.61 & NA & 0.47 & 0.80 & 0.80 & 0.53 & NA \\
\hline $30-40$ & NA & 0.63 & 0.54 & 0.90 & NA & 1.19 & $0.65 *$ & 1.43 & 2.28 & NA \\
\hline $40-50$ & 3.98 & 3.79 & 5.39 & 3.13 & 10.61 & $9.42 *$ & $6.88 *$ & $5.01 *$ & $9.36 * *$ & NA \\
\hline $50-60$ & NA & NA & NA & NA & NA & NA & NA & NA & NA & NA \\
\hline
\end{tabular}


TABLE 67: Pedestrian Risk ratio. Road width by neighborhood concept F, continued

\begin{tabular}{l|l|l|l|l|l|l|l|l|l|l|}
\hline $60-70$ & NA & NA & NA & NA & NA & NA & NA & NA & NA & NA \\
\hline$>80$ & NA & NA & NA & NA & NA & NA & NA & NA & NA & NA \\
\hline \hline
\end{tabular}

TABLE 67: Pedestrian Risk ratio. Number of lanes by neighborhood concept D

\begin{tabular}{|c|c|c|c|c|c|c|c|c|c|c|}
\hline \multirow[b]{2}{*}{ \#OF LANES } & \multicolumn{5}{|c|}{ INTERSECTION } & \multicolumn{5}{|c|}{ SEGMENT } \\
\hline & $\mathbf{K}$ & $\mathbf{A}$ & B & $\mathbf{C}$ & $\mathbf{O}$ & $\mathbf{K}$ & $\mathbf{A}$ & B & $\mathbf{C}$ & $\mathbf{O}$ \\
\hline 1 & NA & NA & $0.33 *$ & $0.22 *$ & NA & NA & NA & NA & NA & NA \\
\hline 2 & 1.23 & 0.86 & 1.04 & 1.20 & 1.94 & 0.54 & 0.73 & 0.87 & 0.26 & NA \\
\hline 3 & NA & 0.23 & 0.25 & 0.25 & 0.56 & 0.46 & NA & $0.10 *$ & 0.15 & NA \\
\hline 4 & 3.24 & 3.32 & 2.66 & 2.37 & 1.05 & 3.49 & 3.88 & 3.37 & 4.41 & NA \\
\hline 5 & NA & NA & NA & NA & NA & NA & NA & NA & NA & NA \\
\hline 6 & NA & NA & NA & NA & NA & NA & NA & NA & NA & NA \\
\hline
\end{tabular}


TABLE 68: Pedestrian Risk ratio. Number of lanes by neighborhood concept E

\begin{tabular}{|c|c|c|c|c|c|c|c|c|c|c|}
\hline \multirow[b]{2}{*}{ \#OF LANES } & \multicolumn{5}{|c|}{ INTERSECTION } & \multicolumn{5}{|c|}{ SEGMENT } \\
\hline & $\mathbf{K}$ & $\mathbf{A}$ & B & C & $\mathbf{O}$ & $\mathbf{K}$ & $\mathbf{A}$ & B & $\mathbf{C}$ & $\mathbf{O}$ \\
\hline 1 & NA & NA & $0.39 *$ & $0.55 *$ & NA & NA & NA & NA & $0.40 *$ & NA \\
\hline 2 & 0.49 & 0.47 & 0.56 & 0.43 & 0.70 & 0.79 & 0.71 & 0.77 & 0.85 & 0.35 \\
\hline 3 & 0.68 & 0.50 & 0.62 & 0.85 & $\mathrm{NA}$ & 0.43 & NA & 0.40 & 0.55 & 2.25 \\
\hline 4 & 3.19 & 3.76 & 3.12 & 3.17 & 2.46 & 2.83 & 3.47 & 2.80 & 2.18 & $\mathrm{NA}$ \\
\hline 5 & NA & NA & NA & NA & NA & NA & NA & NA & NA & NA \\
\hline 6 & NA & NA & NA & NA & NA & NA & NA & NA & NA & NA \\
\hline
\end{tabular}

TABLE 69: Pedestrian Risk ratio. Number of lanes by neighborhood concept $F$

\begin{tabular}{|c|c|c|c|c|c|c|c|c|c|c|}
\hline \multirow[b]{2}{*}{ \#OF LANES } & \multicolumn{5}{|c|}{ INTERSECTION } & \multicolumn{5}{|c|}{ SEGMENT } \\
\hline & $\mathbf{K}$ & $\mathbf{A}$ & B & $\mathbf{C}$ & $\mathbf{O}$ & $\mathbf{K}$ & $\mathbf{A}$ & B & $\mathrm{C}$ & $\mathbf{O}$ \\
\hline 1 & NA & NA & NA & 1.22 & NA & NA & NA & NA & NA & NA \\
\hline 2 & 0.84 & 0.60 & 0.54 & 0.69 & NA & 0.66 & 1.20 & 1.12 & 0.84 & NA \\
\hline 3 & NA & 0.64 & 0.41 & 0.82 & NA & 0.39 & $0.21 *$ & 0.47 & 1.00 & NA \\
\hline 4 & 4.85 & 4.16 & 5.52 & 3.09 & 9.70 & 4.64 & 2.31 & 2.22 & 2.70 & NA \\
\hline 5 & NA & NA & NA & NA & NA & NA & NA & NA & NA & NA \\
\hline 6 & NA & NA & NA & NA & NA & NA & NA & NA & NA & NA \\
\hline
\end{tabular}


TABLE 70: Pedestrian Risk ratio. Road classification by neighborhood concept D

\begin{tabular}{|c|c|c|c|c|c|c|c|c|c|c|}
\hline \multirow[b]{2}{*}{ ROAD CLASSIFICATION } & \multicolumn{5}{|c|}{ INTERSECTION } & \multicolumn{5}{|c|}{ SEGMENT } \\
\hline & $\mathbf{K}$ & A & $\mathbf{B}$ & $\mathbf{C}$ & $\mathbf{O}$ & $\mathbf{K}$ & A & B & $\mathbf{C}$ & $\mathbf{O}$ \\
\hline Interstate & 0.11 & $0.06 *$ & $0.06 *$ & $0.04 *$ & 0.28 & 0.23 & NA & 0.10 & NA & NA \\
\hline Principal Arterial & 3.71 & 3.43 & 3.26 & 3.41 & 3.38 & 3.52 & 3.81 & 3.32 & 3.33 & 3.33 \\
\hline Minor Arterial & $0.92 * * *$ & $2.66 *$ & $2.76 *$ & $2.33 *$ & NA & NA & $0.74 \quad * *$ & $2.87 *$ & $3.02 *$ & $3.02 *$ \\
\hline Major collector & NA & NA & NA & NA & NA & NA & NA & NA & $\mathrm{NA}$ & NA \\
\hline Minor collector & NA & NA & NA & NA & NA & NA & NA & NA & NA & NA \\
\hline Local & NA & NA & NA & NA & NA & NA & NA & NA & NA & NA \\
\hline
\end{tabular}

TABLE 71: Pedestrian Risk ratio. Road classification by neighborhood concept $E$

\begin{tabular}{|c|c|c|c|c|c|c|c|c|c|c|}
\hline \multirow[b]{2}{*}{ ROAD CLASSIFICATION } & \multicolumn{5}{|c|}{ INTERSECTION } & \multicolumn{5}{|c|}{ SEGMENT } \\
\hline & $\mathbf{K}$ & $\mathbf{A}$ & B & $\mathbf{C}$ & $\mathbf{O}$ & $\mathbf{K}$ & $\mathbf{A}$ & B & $\mathbf{C}$ & $\mathbf{O}$ \\
\hline Interstate & 0.14 & $0.05 *$ & 0.13 & 0.12 & 0.34 & 0.19 & 0.14 & 0.09 & 0.22 & 0.34 \\
\hline Principal Arterial & 2.45 & 2.29 & 2.34 & 2.39 & 1.84 & 2.45 & 2.12 & 2.37 & 2.12 & 1.23 \\
\hline Minor Arterial & 1.16 & 2.17 & 1.14 & 1.39 & $\mathrm{NA}$ & 1.34 & 2.34 & 2.04 & 1.64 & 2.91 \\
\hline Major collector & NA & NA & NA & NA & NA & NA & NA & NA & NA & NA \\
\hline Minor collector & NA & NA & NA & NA & NA & NA & NA & NA & NA & NA \\
\hline Local & NA & NA & NA & NA & NA & NA & NA & NA & NA & NA \\
\hline
\end{tabular}


TABLE 72: Pedestrian Risk ratio. Road classification by neighborhood concept $F$

\begin{tabular}{|c|c|c|c|c|c|c|c|c|c|c|}
\hline \multirow[b]{2}{*}{ ROAD CLASSIFICATION } & \multicolumn{5}{|c|}{ INTERSECTION } & \multicolumn{5}{|c|}{ SEGMENT } \\
\hline & $\mathbf{K}$ & $\mathbf{A}$ & B & $\mathbf{C}$ & $\mathbf{O}$ & $\mathbf{K}$ & $\mathbf{A}$ & B & $\mathbf{C}$ & $\mathbf{O}$ \\
\hline Interstate & 0.21 & 0.24 & 0.13 & $0.08 *$ & NA & 0.22 & 0.16 & 0.24 & 0.28 & NA \\
\hline Principal Arterial & 1.82 & 2.60 & 2.75 & 2.82 & NA & 2.06 & 2.78 & 1.75 & 2.23 & NA \\
\hline Minor Arterial & 2.71 & 0.78 & 1.34 & 1.23 & NA & 2.83 & 1.55 & 2.94 & 2.41 & NA \\
\hline Major collector & $\mathrm{NA}$ & $\mathrm{NA}$ & NA & NA & $\mathrm{NA}$ & $\mathrm{NA}$ & $\mathrm{NA}$ & NA & $\mathrm{NA}$ & NA \\
\hline Minor collector & NA & NA & $\mathrm{NA}$ & NA & $\mathrm{NA}$ & NA & $\mathrm{NA}$ & NA & $\mathrm{NA}$ & NA \\
\hline Local & NA & NA & NA & NA & NA & NA & NA & NA & NA & NA \\
\hline
\end{tabular}




\section{B. Bicyclist exposure analysis by neighborhood concept}

TABLE 73: Bicyclist Risk ratio. Posted Speed limit by neighborhood concept D

\begin{tabular}{l|l|l|l|l|l|l|l|l|l|l|l}
\hline \hline & \multicolumn{9}{|c}{ INTERSECTION } & \multicolumn{1}{c}{ SEGMENT } \\
\hline POSTED SPEED LIMIT & $\mathbf{K}$ & $\mathbf{A}$ & $\mathbf{B}$ & $\mathbf{C}$ & $\mathbf{O}$ & $\mathbf{K}$ & $\mathbf{A}$ & $\mathbf{B}$ & $\mathbf{C}$ & O \\
\hline$<20$ & NA & NA & NA & NA & NA & NA & NA & NA & NA & NA \\
\hline $20-35$ & NA & 2.66 & 3.99 & 3.99 & 4.02 & 4.29 & 8.58 & 2.93 & 2.58 & NA \\
\hline $35-50$ & 3.96 & 2.46 & 2.04 & 1.99 & 2.23 & 1.32 & NA & 2.52 & 2.77 & 3.96 \\
\hline $50-65$ & NA & 0.27 & 0.27 & 0.29 & 0.19 & 0.51 & 0.51 & 0.21 & 0.15 & NA \\
\hline$>65$ & NA & NA & NA & NA & NA & NA & NA & NA & NA & NA \\
\hline \hline
\end{tabular}

TABLE 74: Bicyclist Risk ratio. Posted Speed limit by neighborhood concept $E$

\begin{tabular}{|c|c|c|c|c|c|c|c|c|c|c|}
\hline & \multicolumn{5}{|c|}{ INTERSECTION } & \multicolumn{5}{|c|}{ SEGMENT } \\
\hline POSTED SPEED LIMIT & $\mathbf{K}$ & $\mathbf{A}$ & B & $\mathbf{C}$ & $\mathbf{O}$ & $\mathbf{K}$ & $\mathbf{A}$ & B & $\mathbf{C}$ & $\mathbf{O}$ \\
\hline$<20$ & NA & NA & NA & NA & NA & NA & NA & NA & NA & NA \\
\hline $20-35$ & $13.71 *$ & $9.88 *$ & 10.79 & $9.14 *$ & $5.22 *$ & NA & NA & 6.85 & $12.80 *$ & NA \\
\hline $35-50$ & 4.51 & 4.10 & 3.99 & 4.14 & 5.50 & 2.71 & 4.33 & 3.91 & 3.25 & 3.61 \\
\hline $50-65$ & NA & 0.28 & 0.28 & 0.35 & 0.19 & 1.27 & 0.81 & 0.51 & 0.41 & 1.02 \\
\hline$>65$ & NA & 0.08 & NA & NA & NA & NA & NA & 0.06 & NA & NA \\
\hline
\end{tabular}


TABLE 75: Bicyclist Risk ratio. Posted Speed limit by neighborhood concept F

\begin{tabular}{|c|c|c|c|c|c|c|c|c|c|c|}
\hline \multirow[b]{2}{*}{ POSTED SPEED LIMIT } & \multicolumn{5}{|c|}{ INTERSECTION } & \multicolumn{5}{|c|}{ SEGMENT } \\
\hline & $\mathbf{K}$ & $\mathbf{A}$ & B & $\mathbf{C}$ & $\mathbf{O}$ & $\mathbf{K}$ & $\mathbf{A}$ & B & $\mathbf{C}$ & $\mathbf{O}$ \\
\hline$<20$ & NA & NA & NA & NA & NA & NA & NA & NA & NA & NA \\
\hline $20-35$ & NA & NA & NA & NA & NA & NA & NA & NA & NA & NA \\
\hline $35-50$ & NA & 13.09 & 8.28 & 6.82 & 6.55 & 6.55 & 4.36 & 5.54 & 4.36 & NA \\
\hline $50-65$ & NA & NA & 0.32 & 0.54 & 0.99 & 0.99 & 1.32 & 0.84 & 1.32 & 1.98 \\
\hline$>65$ & NA & NA & NA & NA & NA & NA & NA & NA & NA & NA \\
\hline
\end{tabular}

TABLE 76: Bicyclist Risk ratio. Road width by neighborhood concept D

\begin{tabular}{|c|c|c|c|c|c|c|c|c|c|c|}
\hline \multirow[b]{2}{*}{$\begin{array}{l}\text { WIDTH } \\
\text { (ft.) }\end{array}$} & \multicolumn{5}{|c|}{ INTERSECTION } & \multicolumn{5}{|c|}{ SEGMENT } \\
\hline & $\mathbf{K}$ & $\mathbf{A}$ & B & $\mathrm{C}$ & $\mathbf{O}$ & $\mathbf{K}$ & $\mathbf{A}$ & B & $\mathbf{C}$ & $\mathbf{O}$ \\
\hline $0-10$ & NA & NA & NA & NA & NA & NA & NA & NA & NA & NA \\
\hline $10-20$ & NA & $0.31 *$ & 0.51 & $0.41 *$ & NA & NA & NA & NA & NA & NA \\
\hline $20-30$ & 3.30 & 0.57 & 0.89 & 0.89 & 0.82 & NA & 1.10 & 0.60 & 0.33 & NA \\
\hline $30-40$ & NA & 0.27 & 0.42 & 0.29 & 0.17 & 0.88 & 1.76 & 0.36 & 0.26 & NA \\
\hline $40-50$ & NA & 2.64 & 2.19 & 2.42 & 2.58 & 3.93 & NA & 3.48 & 3.53 & 5.89 \\
\hline $50-60$ & NA & $7.02 *$ & $4.32 *$ & $5.36 *$ & $10.17 *$ & NA & NA & 1.85 & $4.07 \quad *$ & NA \\
\hline $60-70$ & NA & NA & NA & NA & NA & NA & NA & NA & NA & NA \\
\hline$>80$ & NA & NA & NA & NA & NA & NA & NA & NA & NA & NA \\
\hline
\end{tabular}


TABLE 77: Bicyclist Risk ratio. Road width by neighborhood concept $\mathbf{E}$

\begin{tabular}{|c|c|c|c|c|c|c|c|c|c|c|}
\hline \multirow[b]{2}{*}{$\begin{array}{l}\text { WIDTH } \\
\text { (ft.) }\end{array}$} & \multicolumn{5}{|c|}{ INTERSECTION } & \multicolumn{5}{|c|}{ SEGMENT } \\
\hline & $\mathbf{K}$ & $\mathbf{A}$ & B & $\mathbf{C}$ & $\mathbf{O}$ & $\mathbf{K}$ & $\mathbf{A}$ & B & $\mathrm{C}$ & $\mathbf{O}$ \\
\hline $0-10$ & NA & NA & NA & NA & NA & NA & NA & NA & NA & NA \\
\hline $10-20$ & NA & NA & $0.57 *$ & 0.70 & 1.22 & NA & NA & NA & NA & 6.40 \\
\hline $20-30$ & 0.45 & 0.43 & 0.49 & 0.41 & 0.42 & 1.34 & 1.25 & 0.48 & 0.71 & NA \\
\hline $30-40$ & 0.68 & 0.59 & 0.60 & 0.60 & $0.26 *$ & NA & 1.09 & 0.57 & NA & NA \\
\hline $40-50$ & 4.05 & 3.51 & 2.85 & 2.93 & 3.40 & 1.62 & 0.65 & 3.11 & 3.24 & 3.24 \\
\hline $50-60$ & NA & NA & NA & NA & NA & NA & NA & NA & NA & NA \\
\hline $60-70$ & NA & NA & NA & NA & NA & NA & NA & NA & NA & NA \\
\hline$>80$ & NA & NA & NA & NA & NA & NA & NA & NA & NA & NA \\
\hline
\end{tabular}

TABLE 78: Bicyclist Risk ratio. Road width by neighborhood concept $F$

\begin{tabular}{|c|c|c|c|c|c|c|c|c|c|c|}
\hline \multirow[b]{2}{*}{$\begin{array}{l}\text { WIDTH } \\
\text { (ft.) }\end{array}$} & \multicolumn{5}{|c|}{ INTERSECTION } & \multicolumn{5}{|c|}{ SEGMENT } \\
\hline & $\mathbf{K}$ & $\mathbf{A}$ & B & C & $\mathbf{O}$ & $\mathbf{K}$ & $\mathbf{A}$ & B & $\mathbf{C}$ & $\mathbf{O}$ \\
\hline $0-10$ & NA & NA & NA & NA & NA & NA & NA & NA & NA & NA \\
\hline $10-20$ & NA & NA & $0.62 *$ & $0.29 *$ & NA & NA & NA & $0.53 *$ & 2.29 & NA \\
\hline $20-30$ & 1.68 & 0.67 & 0.48 & 0.49 & 0.56 & 1.68 & 1.68 & 0.71 & 0.84 & 1.68 \\
\hline $30-40$ & NA & NA & 0.53 & 0.46 & NA & NA & $\mathrm{NA}$ & 0.68 & $\mathrm{NA}$ & $\mathrm{NA}$ \\
\hline $40-50$ & NA & 6.37 & 4.91 & 5.53 & 7.07 & NA & NA & 4.08 & 3.54 & NA \\
\hline $50-60$ & NA & NA & NA & NA & NA & NA & NA & NA & NA & NA \\
\hline
\end{tabular}

s 
TABLE 78: Bicyclist Risk ratio. Road width by neighborhood concept $F$, continued

\begin{tabular}{l|l|l|l|l|l|l|l|l|l|l|}
\hline $60-70$ & NA & NA & NA & NA & NA & NA & NA & NA & NA & NA \\
\hline$>80$ & NA & NA & NA & NA & NA & NA & NA & NA & NA & NA \\
\hline \hline
\end{tabular}

TABLE 79: Bicyclist Risk ratio. Number of lanes by neighborhood concept D

\begin{tabular}{|c|c|c|c|c|c|c|c|c|c|c|}
\hline \multirow[b]{2}{*}{ \#OF LANES } & \multicolumn{5}{|c|}{ INTERSECTION } & \multicolumn{5}{|c|}{ SEGMENT } \\
\hline & $\mathbf{K}$ & $\mathbf{A}$ & B & $\mathbf{C}$ & $\mathbf{O}$ & $\mathbf{K}$ & $\mathbf{A}$ & $\mathbf{B}$ & $\mathbf{C}$ & $\mathbf{O}$ \\
\hline 1 & NA & $0.26 *$ & 0.50 & $0.36 *$ & NA & NA & NA & NA & NA & NA \\
\hline 2 & 3.23 & 0.78 & 1.19 & 1.05 & 0.81 & NA & 2.15 & 0.59 & 0.32 & NA \\
\hline 3 & NA & $0.10 *$ & 0.26 & 0.19 & 0.17 & 0.93 & 0.93 & 0.38 & 0.28 & NA \\
\hline 4 & NA & 3.25 & 2.20 & 2.68 & 3.60 & 3.49 & NA & 3.33 & 3.67 & 5.24 \\
\hline 5 & NA & NA & NA & NA & NA & NA & NA & NA & NA & NA \\
\hline 6 & NA & NA & NA & NA & NA & NA & NA & NA & NA & NA \\
\hline
\end{tabular}


TABLE 80: Bicyclist Risk ratio. Number of lanes by neighborhood concept $\mathbf{E}$

\begin{tabular}{|c|c|c|c|c|c|c|c|c|c|c|}
\hline \multirow[b]{2}{*}{ \#OF LANES } & \multicolumn{5}{|c|}{ INTERSECTION } & \multicolumn{5}{|c|}{ SEGMENT } \\
\hline & $\mathbf{K}$ & $\mathbf{A}$ & B & C & $\mathbf{O}$ & $\mathbf{K}$ & $\mathbf{A}$ & B & $\mathbf{C}$ & $\mathbf{O}$ \\
\hline 1 & NA & NA & 0.63 & 0.67 & 1.17 & NA & NA & NA & NA & 6.13 \\
\hline 2 & 0.44 & 0.57 & 0.56 & 0.54 & 0.42 & 1.32 & 1.23 & 0.59 & 0.70 & NA \\
\hline 3 & 1.41 & $0.15 *$ & 0.52 & 0.31 & 0.54 & 0.70 & 1.13 & 0.35 & NA & 2.82 \\
\hline 4 & 3.07 & 3.65 & 3.01 & 3.15 & 3.22 & 0.77 & 0.61 & 3.58 & 3.68 & NA \\
\hline 5 & NA & NA & $\mathrm{NA}$ & NA & $\mathrm{NA}$ & NA & $\mathrm{NA}$ & $\mathrm{NA}$ & $\mathrm{NA}$ & $\mathrm{NA}$ \\
\hline 6 & NA & NA & NA & NA & NA & NA & NA & NA & NA & NA \\
\hline
\end{tabular}

TABLE 81: Bicyclist Risk ratio. Number of lanes by neighborhood concept F

\begin{tabular}{|c|c|c|c|c|c|c|c|c|c|c|}
\hline \multirow[b]{2}{*}{ \#OF LANES } & \multicolumn{5}{|c|}{ INTERSECTION } & \multicolumn{5}{|c|}{ SEGMENT } \\
\hline & $\mathbf{K}$ & $\mathbf{A}$ & B & $\mathbf{C}$ & $\mathbf{O}$ & $\mathbf{K}$ & $\mathbf{A}$ & B & $\mathbf{C}$ & $\mathbf{O}$ \\
\hline 1 & NA & NA & $0.60 *$ & $0.28 *$ & NA & NA & NA & $0.51 *$ & 2.23 & NA \\
\hline 2 & 1.68 & 0.67 & 0.55 & 0.56 & 0.56 & 1.68 & 1.68 & 0.77 & 0.84 & 1.68 \\
\hline 3 & NA & NA & 0.47 & 0.56 & NA & NA & NA & 0.52 & NA & NA \\
\hline 4 & NA & 5.82 & 4.63 & 4.65 & 6.47 & NA & NA & 3.73 & 3.23 & NA \\
\hline 5 & NA & NA & NA & NA & NA & NA & NA & NA & NA & NA \\
\hline 6 & NA & NA & NA & NA & NA & NA & NA & NA & NA & NA \\
\hline
\end{tabular}


TABLE 82: Bicyclist Risk ratio. Road classification by neighborhood concept D

\begin{tabular}{|c|c|c|c|c|c|c|c|c|c|c|}
\hline \multirow[b]{2}{*}{ ROAD CLASSIFICATION } & \multicolumn{5}{|c|}{ INTERSECTION } & \multicolumn{5}{|c|}{$\overline{\text { SEGMENT }}$} \\
\hline & $\mathbf{K}$ & $\mathbf{A}$ & B & $\mathbf{C}$ & $\mathbf{O}$ & $\mathbf{K}$ & $\mathbf{A}$ & B & $\mathbf{C}$ & $\mathbf{O}$ \\
\hline Interstate & NA & 0.10 & $0.05 *$ & $0.04 *$ & NA & 0.47 & 0.47 & NA & NA & NA \\
\hline Principal Arterial & 4.22 & 3.06 & 2.93 & 3.04 & 3.17 & 2.81 & 2.81 & 3.07 & 3.80 & 4.22 \\
\hline Minor Arterial & NA & $3.16 *$ & $4.02 *$ & $3.38 *$ & $5.73 *$ & NA & NA & $3.13 *$ & NA & NA \\
\hline Major collector & NA & NA & NA & NA & NA & NA & NA & NA & NA & NA \\
\hline Minor collector & NA & NA & NA & NA & NA & NA & NA & NA & NA & NA \\
\hline Local & NA & NA & NA & NA & NA & NA & NA & NA & NA & NA \\
\hline
\end{tabular}

TABLE 83: Bicyclist Risk ratio. Road classification by neighborhood concept $\mathbf{E}$

\begin{tabular}{|c|c|c|c|c|c|c|c|c|c|c|}
\hline \multirow[b]{2}{*}{ ROAD CLASSIFICATION } & \multicolumn{5}{|c|}{ INTERSECTION } & \multicolumn{5}{|c|}{ SEGMENT } \\
\hline & $\mathbf{K}$ & $\mathbf{A}$ & B & $\mathbf{C}$ & $\mathbf{O}$ & $\mathbf{K}$ & $\mathbf{A}$ & B & $\mathrm{C}$ & $\mathbf{O}$ \\
\hline Interstate & NA & NA & $0.06 *$ & $0.05 *$ & 0.25 & NA & NA & $0.04 *$ & NA & NA \\
\hline Principal Arterial & 3.06 & 2.47 & 2.32 & 2.24 & 2.19 & 1.91 & 2.14 & 2.55 & 2.45 & 3.06 \\
\hline Minor Arterial & NA & 2.02 & 1.80 & 2.05 & $0.69 *$ & 3.63 & 2.91 & 1.51 & 1.45 & NA \\
\hline Major collector & NA & NA & NA & NA & NA & NA & NA & NA & NA & NA \\
\hline Minor collector & NA & NA & NA & NA & NA & NA & NA & NA & NA & NA \\
\hline Local & NA & NA & NA & NA & NA & NA & NA & NA & NA & NA \\
\hline
\end{tabular}


TABLE 84: Bicyclist Risk ratio. Road classification by neighborhood concept $\mathbf{F}$

\begin{tabular}{|c|c|c|c|c|c|c|c|c|c|c|}
\hline \multirow[b]{2}{*}{ ROAD CLASSIFICATION } & \multicolumn{5}{|c|}{ INTERSECTION } & \multicolumn{5}{|c|}{ SEGMENT } \\
\hline & $\mathbf{K}$ & $\mathbf{A}$ & B & $\mathbf{C}$ & $\mathbf{O}$ & $\mathbf{K}$ & $\mathbf{A}$ & B & $\mathbf{C}$ & $\mathbf{O}$ \\
\hline Interstate & NA & NA & $0.05 *$ & $0.07 *$ & NA & NA & NA & NA & NA & NA \\
\hline Principal Arterial & NA & 2.92 & 2.45 & 2.66 & 3.65 & 1.82 & 0.61 & 2.52 & 2.43 & 3.65 \\
\hline Minor Arterial & 10.85 & 2.17 & 1.94 & 0.90 & NA & 5.43 & 5.43 & 3.34 & 1.81 & NA \\
\hline Major collector & NA & NA & NA & NA & NA & NA & NA & NA & NA & NA \\
\hline Minor collector & NA & NA & NA & NA & NA & NA & $\mathrm{NA}$ & NA & NA & NA \\
\hline Local & NA & NA & NA & NA & NA & NA & NA & NA & NA & NA \\
\hline
\end{tabular}




\section{Appendix B: Pooled Models}

TABLE 85: Detailed ordinal regression model. Crash severity for pedestrians and bicyclists

\begin{tabular}{|c|c|c|c|c|c|c|c|c|c|c|}
\hline \multirow[t]{2}{*}{ VARIABLE } & \multirow{2}{*}{$\begin{array}{c}\text { BASE } \\
\text { GROUP }\end{array}$} & \multirow[t]{2}{*}{ LEVEL } & \multicolumn{4}{|c|}{ PEDESTRIAN } & \multicolumn{4}{|c|}{ BICYCLIST } \\
\hline & & & $\begin{array}{c}\text { PAR } \\
\text {. }\end{array}$ & $\begin{array}{c}\text { STD } \\
\text { ERR } \\
\text { OR }\end{array}$ & $\begin{array}{c}\mathbf{Z} \\
\text { VAL } \\
\text { UE }\end{array}$ & $\begin{array}{c}\mathbf{P} \\
\text { VAL } \\
\text { UE }\end{array}$ & $\begin{array}{c}\text { PAR } \\
\text {. }\end{array}$ & $\begin{array}{c}\text { STD } \\
\text { ERR } \\
\text { OR }\end{array}$ & $\begin{array}{c}\mathbf{Z} \\
\text { VAL } \\
\text { UE }\end{array}$ & $\begin{array}{c}\mathbf{P} \\
\text { VAL } \\
\text { UE }\end{array}$ \\
\hline Light conditions & $\begin{array}{l}\text { Other lighting } \\
\text { conditions }\end{array}$ & $\begin{array}{c}\text { Darkness no } \\
\text { street light }\end{array}$ & 0.67 & 0.15 & 4.55 & $* * *$ & NA & & & \\
\hline $\begin{array}{c}\text { Alcohol } \\
\text { intoxication }\end{array}$ & No & Yes & 0.91 & 0.14 & 6.46 & $* * *$ & NA & & & \\
\hline $\begin{array}{c}\text { Vehicle } \\
\text { movement }\end{array}$ & Straight & Turning & -0.72 & 0.12 & -6.01 & $* * *$ & -0.41 & 0.15 & -2.78 & $* *$ \\
\hline Vehicle type & $\begin{array}{l}\text { Passenger } \\
\text { vehicle }\end{array}$ & $\begin{array}{l}\text { Truck and } \\
\text { buses }\end{array}$ & 0.91 & 0.32 & 2.88 & $* *$ & NA & & & \\
\hline $\begin{array}{c}\text { Road } \\
\text { classification }\end{array}$ & Arterials & Local streets & -0.74 & 0.30 & -2.48 & $*$ & NA & & & \\
\hline $\begin{array}{l}\text { Traffic control } \\
\text { Device }\end{array}$ & Other devices & $\begin{array}{c}\text { Special } \\
\text { pedestrian } \\
\text { signal }\end{array}$ & 0.73 & 0.28 & 2.60 & $* *$ & NA & & & \\
\hline
\end{tabular}

$\bar{\infty}$ 
TABLE 85: Detailed ordinal regression model. Crash severity for pedestrians and bicyclists, continued

\begin{tabular}{|c|c|c|c|c|c|c|c|c|c|c|}
\hline Age & $\leq 54$ & $55-74$ & 0.50 & 0.13 & 3.85 & $* * *$ & 0.65 & 0.19 & 3.36 & $* * *$ \\
\hline & & $>74$ & 1.60 & 0.23 & 6.97 & $* * *$ & 2.23 & 0.50 & 4.45 & $* * *$ \\
\hline $\begin{array}{l}\text { Posted speed } \\
\text { limit }\end{array}$ & $<50 \mathrm{mph}$ & $\geq 50 \mathrm{mph}$ & 0.46 & 0.18 & 2.57 & $*$ & NA & & & \\
\hline Location & Intersection & Segment & NA & & & & 0.57 & 0.22 & 2.64 & $* *$ \\
\hline Pedestrian/Bicy & Crosswalk & Roadway & 0.36 & 0.13 & 2.73 & $* *$ & NA & & & \\
\hline $\begin{array}{l}\text { clist location } \\
\text { during the crash }\end{array}$ & & Midblock & -0.86 & 0.32 & -2.70 & $* *$ & NA & & & \\
\hline $\begin{array}{l}\text { AADT (only } \\
\text { truck) }\end{array}$ & $\leq 670$ & $>670$ & 0.31 & 0.11 & 2.79 & $* *$ & & & & \\
\hline AADT & $\leq 10,000$ & $>10,000$ & NA & & & & -0.31 & 0.15 & -2.03 & $*$ \\
\hline $\begin{array}{l}\text { Interaction } \\
\text { weather and } \\
\text { road surface } \\
\text { conditions }\end{array}$ & $\begin{array}{l}\text { Other weather } \\
\text { and road } \\
\text { surface } \\
\text { conditions }\end{array}$ & $\begin{array}{l}\text { Wet road } \\
\text { surface and } \\
\text { clear day }\end{array}$ & 0.43 & 0.14 & 3.21 & $* *$ & NA & & & \\
\hline
\end{tabular}

5 
TABLE 85: Detailed ordinal regression model. Crash severity for pedestrians and bicyclists, continued

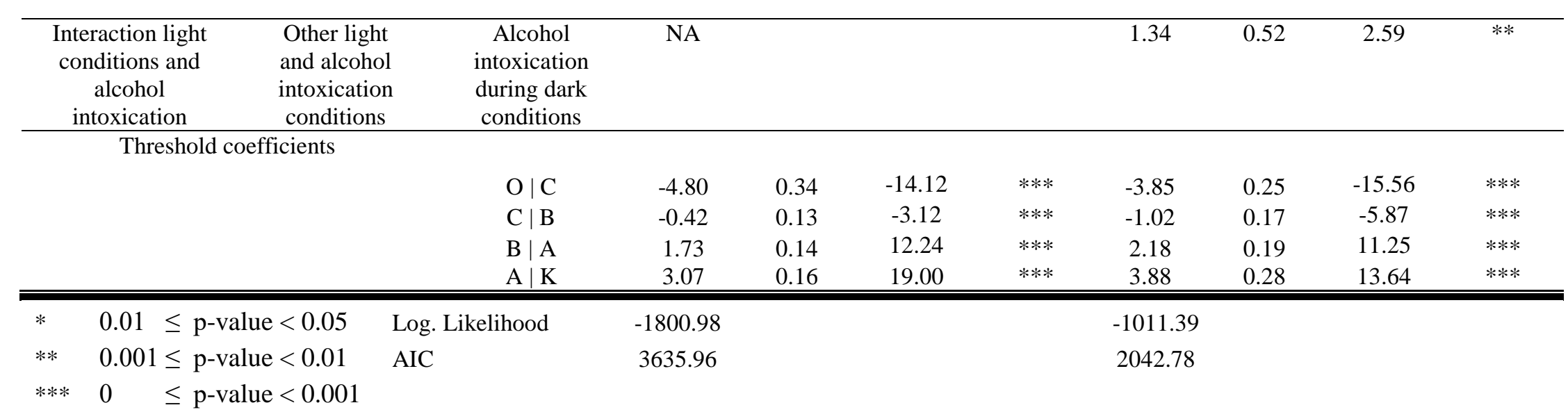




\section{Appendix C: Road characteristics and traffic conditions models}

TABLE 86: Detailed ordinal regression model. Road and traffic characteristics

\begin{tabular}{|c|c|c|c|c|c|c|c|c|c|c|}
\hline \multirow[t]{2}{*}{ VARIABLE } & \multirow{2}{*}{$\begin{array}{c}\text { BASE } \\
\text { GROUP }\end{array}$} & \multirow[t]{2}{*}{ LEVEL } & \multicolumn{4}{|c|}{ PEDESTRIAN } & \multicolumn{4}{|c|}{ BICYCLIST } \\
\hline & & & $\begin{array}{c}\text { PAR } \\
\cdot\end{array}$ & $\begin{array}{c}\text { STD } \\
\text { ERR } \\
\text { OR }\end{array}$ & $\begin{array}{c}\mathbf{Z} \\
\text { VAL } \\
\text { UE }\end{array}$ & $\begin{array}{c}\mathbf{P} \\
\text { VAL } \\
\text { UE }\end{array}$ & $\begin{array}{c}\text { PAR } \\
.\end{array}$ & $\begin{array}{c}\text { STD } \\
\text { ERR } \\
\text { OR }\end{array}$ & $\begin{array}{c}\mathbf{Z} \\
\text { VAL } \\
\text { UE }\end{array}$ & $\begin{array}{c}\text { P } \\
\text { VAL } \\
\text { UE }\end{array}$ \\
\hline Light conditions & $\begin{array}{l}\text { Other lighting } \\
\text { conditions }\end{array}$ & $\begin{array}{c}\text { Darkness no } \\
\text { street light }\end{array}$ & 0.88 & 0.14 & 3.04 & $* * *$ & $\mathrm{NA}$ & & & \\
\hline $\begin{array}{c}\text { Road } \\
\text { classification }\end{array}$ & Arterials & Local streets & -0.76 & 0.29 & -2.58 & $*$ & NA & & & \\
\hline $\begin{array}{l}\text { Posted speed } \\
\text { limit }\end{array}$ & $<50 \mathrm{mph}$ & $\geq 50 \mathrm{mph}$ & 0.47 & 0.17 & 2.69 & $* *$ & NA & & & \\
\hline Location & Intersection & Segment & 0.71 & 0.11 & 6.50 & $* * *$ & 0.86 & 0.20 & 4.39 & $* *$ \\
\hline $\begin{array}{l}\text { AADT (only } \\
\text { truck) }\end{array}$ & $<670$ & $670-1100$ & 0.39 & 0.11 & 3.52 & $* *$ & & & & \\
\hline AADT & $\leq 10,000$ & $>10,000$ & NA & & & & -0.33 & 0.15 & -2.21 & $*$ \\
\hline \multicolumn{11}{|c|}{ Threshold coefficients } \\
\hline & & $\mathrm{O} \mid \mathrm{C}$ & -4.50 & 0.33 & -13.77 & $* * *$ & -3.66 & 0.22 & -16.42 & $* * *$ \\
\hline & & $\mathrm{C} \mid \mathrm{B}$ & -0.21 & 0.10 & -2.05 & $* * *$ & -0.85 & 0.14 & -6.15 & $* * *$ \\
\hline & & $\mathrm{B} \mid \mathrm{A}$ & 1.76 & 0.11 & 15.72 & $* * *$ & 2.22 & 0.16 & 13.67 & $* * *$ \\
\hline & & $\mathrm{A} \mid \mathrm{K}$ & 2.99 & 0.13 & 22.78 & $* * *$ & 3.86 & 0.26 & 14.82 & $* * *$ \\
\hline
\end{tabular}


TABLE 86: Detailed ordinal regression model. Road and traffic characteristics, continued

* $\quad 0.01 \leq$ p-value $<0.05$

** $\quad 0.001 \leq \mathrm{p}$-value $<0.01$

Log. Likelihood

$-1890.68$

AIC

3799.36

$-1033.67$

$* * * \quad 0 \leq \mathrm{p}$-value $<0.001$ 